\title{
TRPV1 regulates excitatory innervation of oriens lacunosum moleculare (OLM) neurons in the hippocampus to affect synaptic plasticity
}

\author{
Dissertation \\ For the award of the degree \\ "Doctor rerum naturalium" (Dr.rer.nat.) \\ Division of Mathematics and Natural Sciences \\ of the Georg-August-Universität Göttingen \\ within the doctoral program of Biology \\ of the Georg-August University School of Science (GAUSS) \\ Submitted by \\ Joaquín Isaac Hurtado Zavala \\ Born in \\ Monterrey, Mexico
}

Göttingen, 18th March 2016 
Thesis committee members:

Camin Dean, PhD (reviewer)

Trans-synaptic Signaling Group, European Neuroscience Institute, Göttingen

Prof. Dr. Ralf Heinrich (reviewer)

Department of Cellular Neurobiology, Schwann-Schleiden Research Centre, Göttingen.

Prof. Dr. Thomas Dresbach

Department of Anatomy, University Medical Center, Göttingen

Extended thesis committee members:

Dr. Manuela Schmidt

Somatosensory Signaling Group, Max Planck Institute for Experimental Medicine, Göttingen

Prof. Dr. Michael Hörner

Department of Cellular Neurobiology, Schwann-Schleiden Research Center, Göttingen

Prof. Dr. Martin Göpfert

Department of Cellular Neurobiology, Schwann-Schleiden Research Center, Göttingen

Date of the oral examination: 13th April 2016 


\section{Declaration:}

I hereby declare that the PhD thesis entitled "TRPV1 regulates excitatory innervation of oriens lacunosum moleculare (OLM) neurons in the hippocampus to affect synaptic plasticity" was written independently and with no other sources and aids than quoted and never been submitted for a degree or other qualification at any other University.

Göttingen, 18th March 2016

Joaquín Isaac Hurtado Zavala 


\section{Acknowledgments:}

I would like to thank PhD. Camin Dean for giving me the great opportunity to join her scientific group and to allow me to conduct this research following my own ideas and observations. With her guidance and help I learned to use my creativity to solve any obstacle as well as to broaden my vision on how to do science in a very professional way. Thank you for your support, absolute patience and meticulous scientific observations that allowed me to complete this scientific research.

Prof. Dr. Ralf Heinrich has been for me since the beginning of my scientific career a role model. I thank him for the absolute support and advice throughout a substantial period of my academic development.

Thanks also to Prof. Dr. Thomas Dresbach for such an active involvement in the thesis committee meetings.

Thanks to all the members of the lab. Some of you became more than lab colleagues. Thanks to Dr. Saheeb Ahmed for the great scientific advice and for helping me out with biochemical assays. Thanks to Ankit Awasthi for being an unconditional helper in time of need and to keep me provided with extra calories needed for the achievement of this successful project. Thanks to Vinita Bharat for constantly remind me that life can be full of joy and happiness. Thanks to Markus Stahlberg for his scientific observations and interesting conversations. Thanks to Dr. Anne Wolfes for all the pens that she allowed me to "steal" from her cabinet, for giving me moral support before starting to write the thesis and her great advises to achieve a nice edition of the thesis. Thanks to Dr. Binu Ramachandran for his pure smiles and for the help that he provided for the LTP and LTD experiments. Thanks to Christiane Bolleyer for being such an outstanding student and for her exceptional collaboration in this research. Thanks to Dr. Yo Shinoda for showing me that lab work is nicer with proper planning and structure. 
Thanks to Dr. Rodolfo Briones, Dr. Vladimir González, Dr. Alfredo Nicolás Erlwein Vicuña, Dr. Christoph Ott, Dr. Jaffer Shahab, Juan Esteban Mora Cid, Juan Torroba and Vince Canger for being an important part of my life, for the unconditional listening and exceptional advice in and beyond my scientific life.

Thanks to Mercedes, Marie, Fabian, Lars, Ronja, Maria, Chrima, Ronnie, Gravy and all of the Gosse crew for being always such good friends and being such a source of understanding and endless joy.

Thanks to all my family, my cousins Abelardo Alanis Zavala and Carmen Julia Alemán Hurtado, you are like brothers to me. I especially thanks my father Joaquín Hurtado Pérez and my mother Rosalinda Zavala Salazar for their absolute support throughout my life and for constantly encourage me to believe in myself and to be confident to achieve all of my goals. This thesis is also a reflection of all your care and attention.

Special thanks to my wife and life partner Johanna Karch. Thanks for all your understanding and patience. Thanks for making me every day the happiest person I could ever imagine to be. This work is completely dedicated to you. 


\section{TABLE OF CONTENTS}

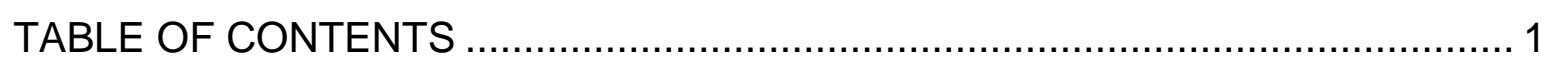

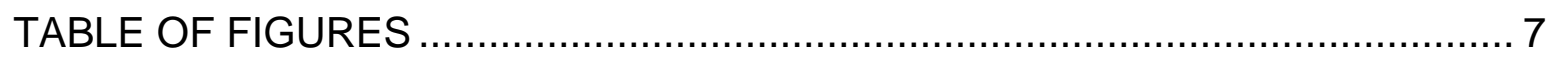

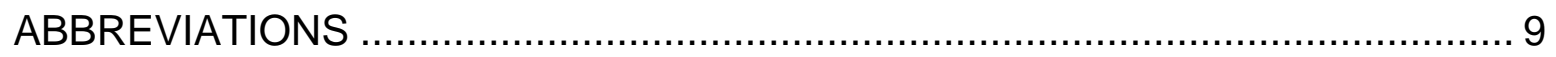

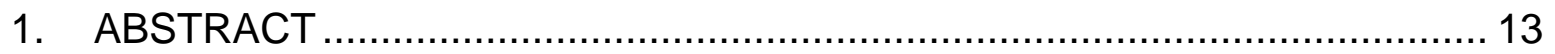

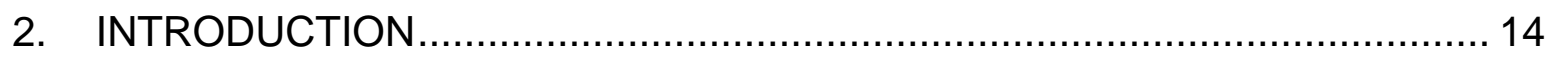

2.1. TRPV1 GENE TRANSCRIPTION AND PROTEIN STRUCTURE ............ 15

2.1.1. The Trpv1 Gene.................................................................... 15

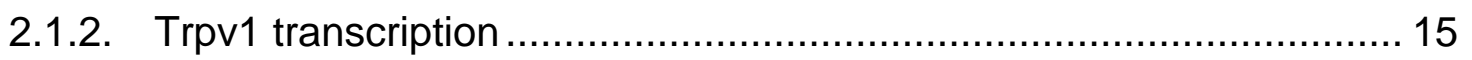

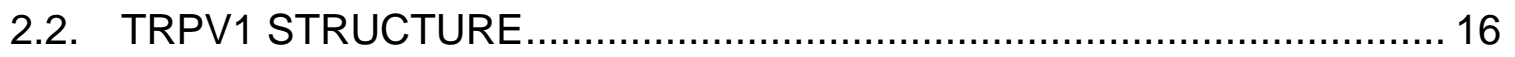

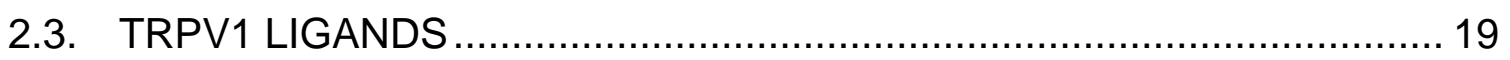

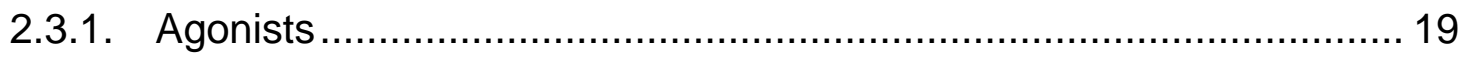

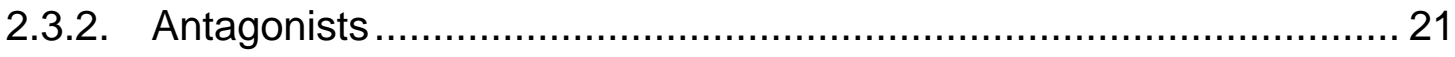

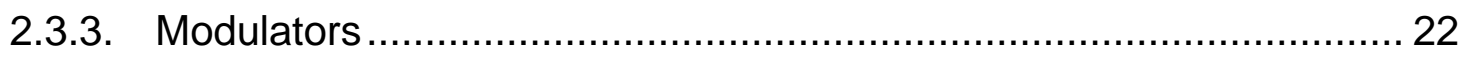

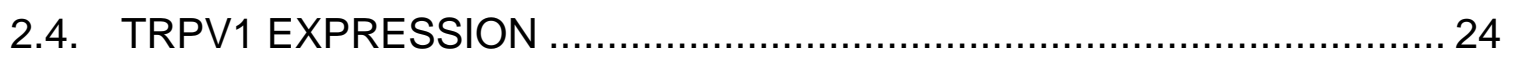

2.5. TRPV1 IN THE BRAIN: PHYSIOLOGICAL RELEVANCE ..................... 26

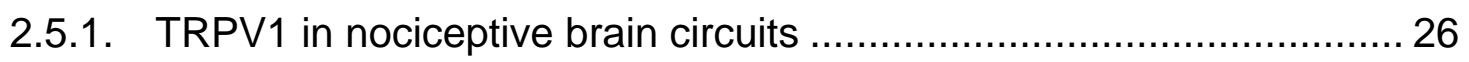

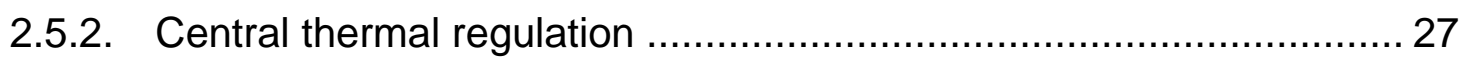

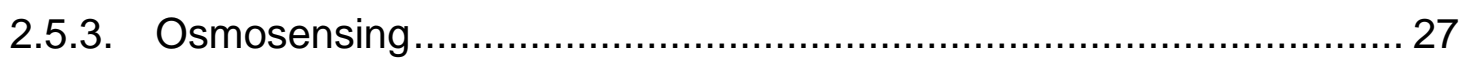

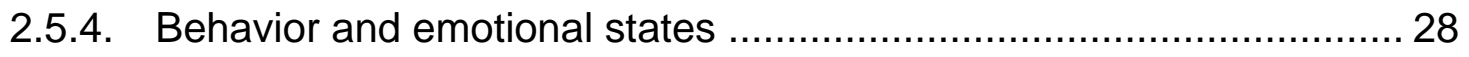

2.6. TRPV1 IN THE HIPPOCAMPUS: EXPRESSION AND ROLE IN

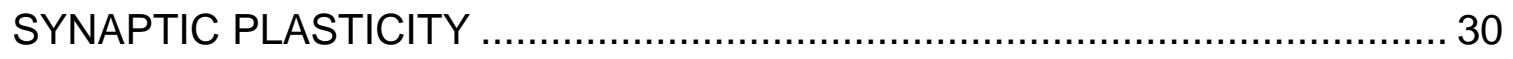

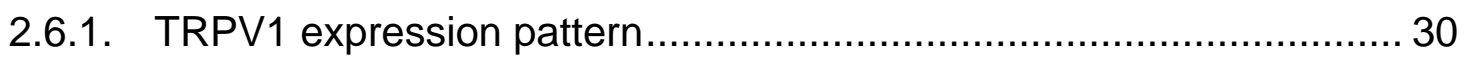




\section{TABLE OF CONTENTS}

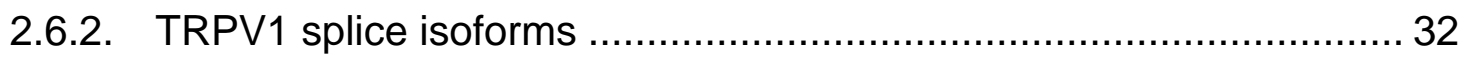

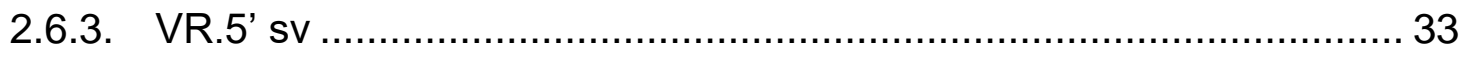

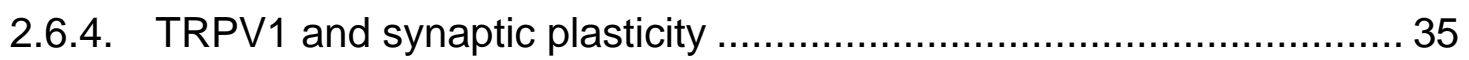

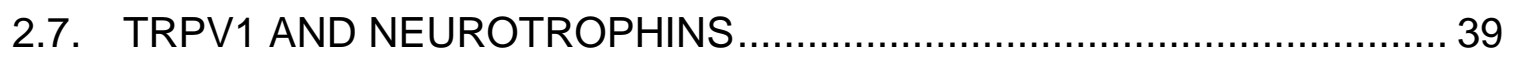

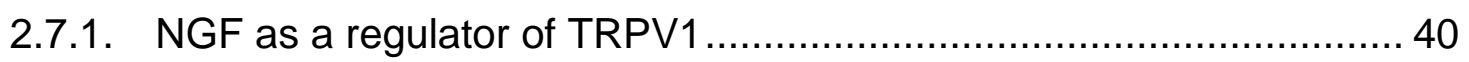

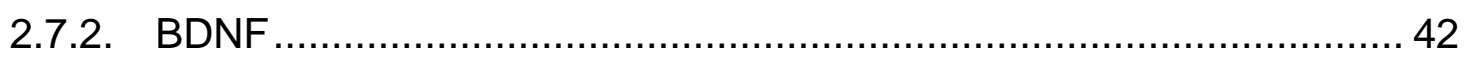

2.8. ORIENS LACUNOSUM MOLECULARE (OLM) INTERNEURONS ......... 44

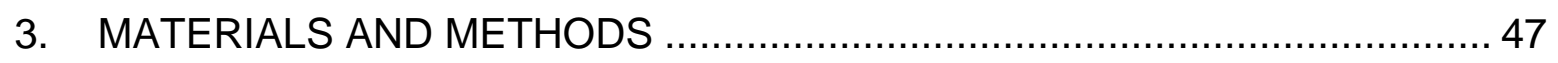

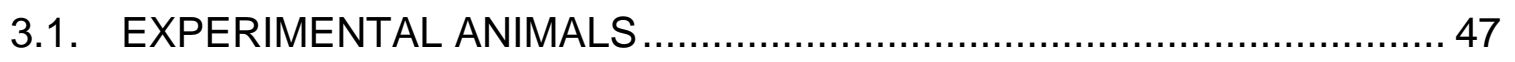

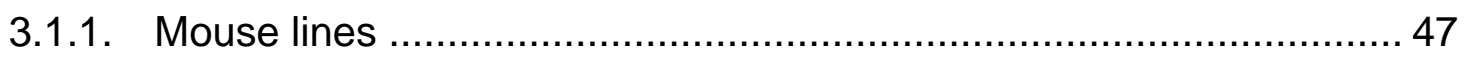

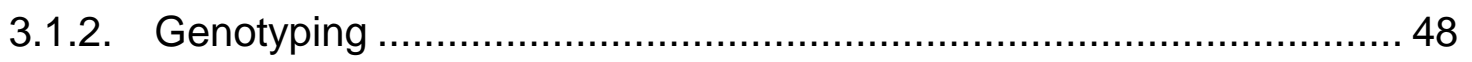

3.2. PLASMIDS: DESIGN AND ISOLATION .............................................. 51

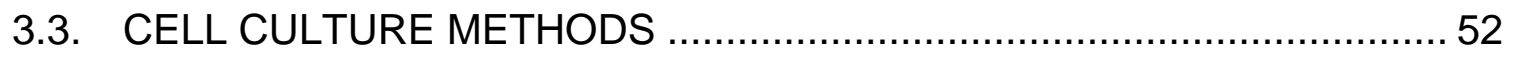

3.3.1. Dissociated rat hippocampal neuron preparation............................ 52

3.3.2. Dissociated mouse hippocampal neuron preparation ....................... 53

3.3.3. Human embryonic kidney 293 (HEK) cell culture preparation ............ 54

3.4. HETEROLOGOUS OVEREXPRESSION OF THE FULL LENGTH TRPV1

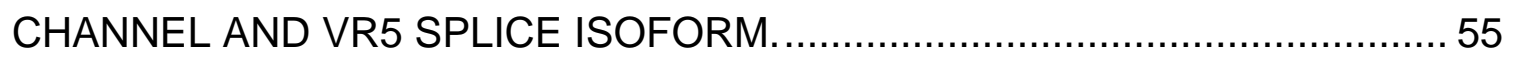

3.4.1. Transfection of dissociated rat hippocampal neurons ...................... 55

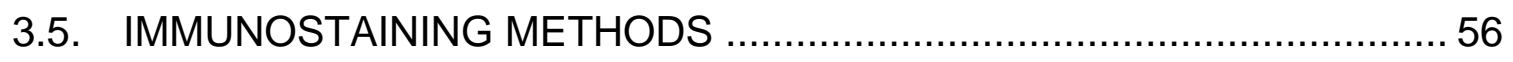

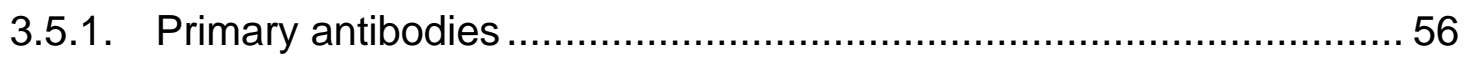

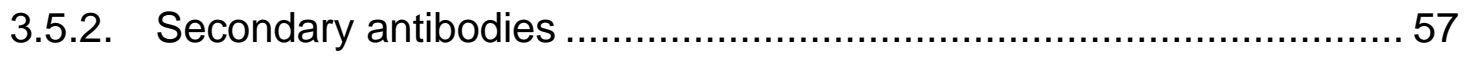

3.5.3. Synaptotagmin 1 luminal domain antibody uptake............................ 58

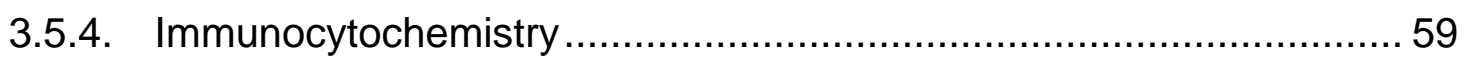

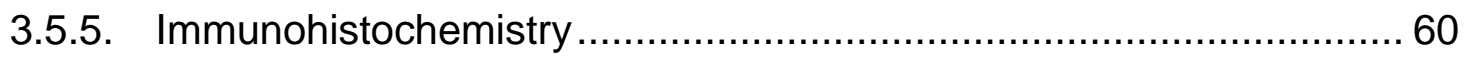




\section{TABLE OF CONTENTS}

3.6. MICROSCOPY: IMAGE ACQUISITION AND ANALYSIS ....................... 62

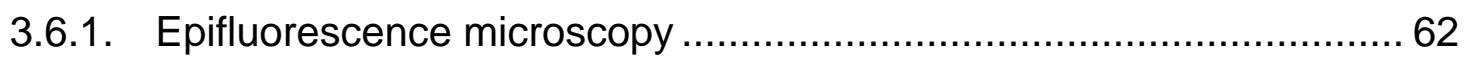

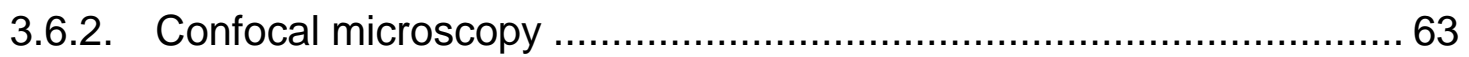

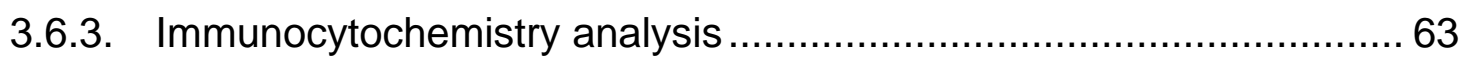

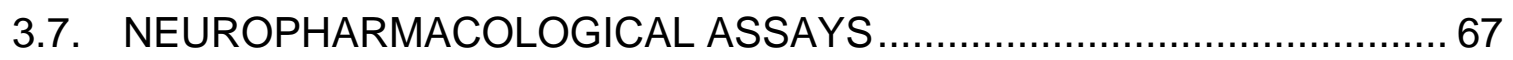

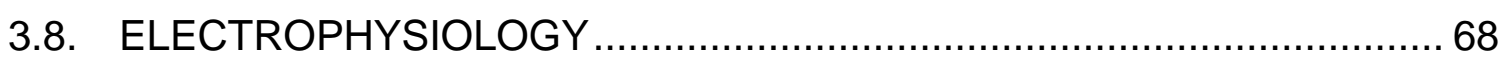

3.8.1. Miniature excitatory and inhibitory postsynaptic current recordings... 68

3.8.2. Electrophysiological recording from hippocampal slices ................... 69

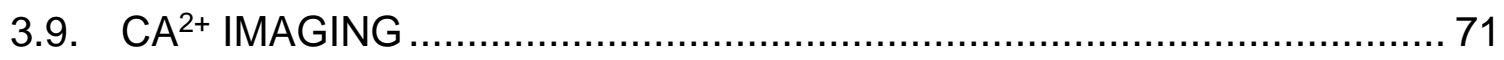

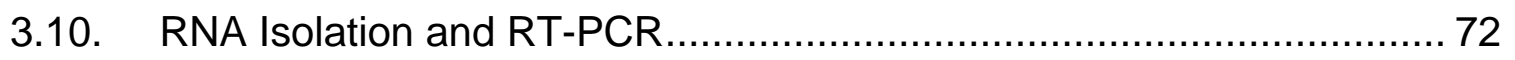

3.10.1. Primer design, PCR and Q-PCR ….......................................... 74

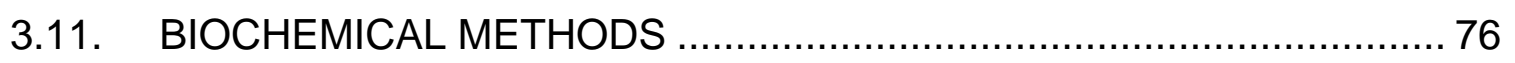

3.11.1. Sample tissue collection and preparation ................................... 76

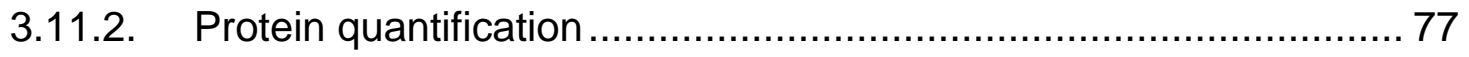

3.11.3. Protein separation (SDS-PAGE) …….................................... 77

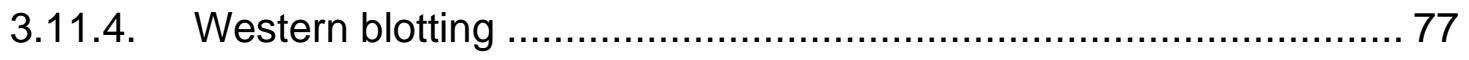

3.11.5. Isolation of synaptic vesicles from mouse brain homogenates ....... 78

3.11.6. Protease treatment of synaptosomes ............................................ 78

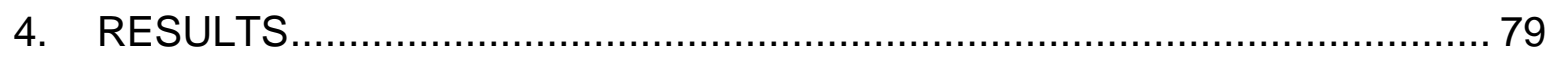

4.1. TRPV1 EXPRESSION IN BRAIN AND HIPPOCAMPUS ....................... 79

4.1.1. TRPV1 and VR.5' sv mRNA are differentially expressed in the hippocampus and DRG from WT and TRPVI KO mice ............................... 80

4.1.2. TRPV1 protein expression levels in the mouse brain and hippocampus 83

4.1.3. Pre- and post-synaptic expression of TRPV1 ................................. 85 


\section{TABLE OF CONTENTS}

4.1.4. Expression of functional TRPV1 in hippocampal neuronal cultures... 86 4.2. TRPV1 IS HIGHLY EXPRESSED IN A SPECIFIC HIPPOCAMPAL

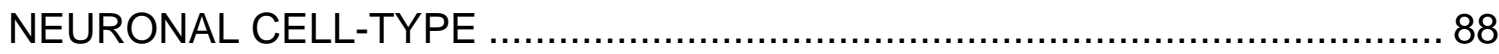

4.2.1. High TRPV1 expressing neurons in the stratum oriens ................... 90

4.2.2. TRPV1 expression in oriens lacunosum moleculare inhibitory interneurons 92

4.3. TRPV1 EXPRESSION IN OLM NEURONS MODULATES EXCITATORY

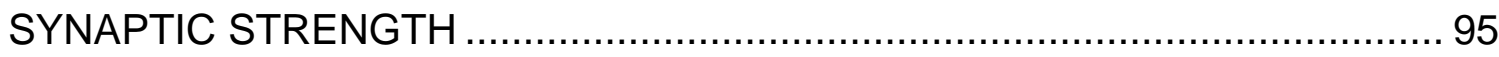

4.3.1. TRPV1 channel activity influences excitatory pre-synaptic strength in OLM neurons 96

4.3.2. TRPV1 expression increases excitatory but not inhibitory synapse innervation in OLM neurons 100

4.4. HETEROLOGOUS TRPV1 OVEREXPRESSION INCREASES

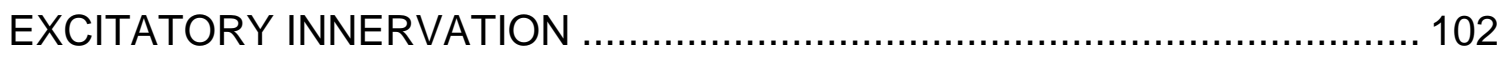

4.4.1. TRPV1 overexpression at DIV2 in hippocampal neurons increases excitatory innervation ......................................................................... 102

4.5. TRPV1 EFFECTS ON SCHAFFER COLLATERAL LTP AND LTD ........ 106 4.5.1. TRPV1 does not affect LTD in the Schaffer collateral pathway of the CA1 region of hippocampus 106

4.5.2. TRPV1-induced innervation of OLM neurons is necessary for gating of CA3 hippocampal information 106

4.6. REGULATION OF TRPV1 EXPRESSION IN HIPPOCAMPAL NEURONS IN VITRO 109

4.7. NGF UPREGULATES TRPV1 EXPRESSION IN HIPPOCAMPAL NEURONS 110

4.7.1. NGF increases TRPV1 expression in hippocampal neurons in vitro 110 4.7.2. High expression of TRPV1 in OLM neurons is driven by NGF 111 


\section{TABLE OF CONTENTS}

4.8. TRPV1 UPREGULATION UPON NEURONAL ACTIVATION: A LINK BETWEEN TRPV1 AND BDNF

4.8.1. TRPV1 overexpression increases BDNF .................................... 114

4.8.2. High TRPV1-expressing neurons have increased BDNF expression 115

4.8.3. Elevated neuronal activity increases both TRPV1 and BDNF levels 116 4.9. DOES BDNF INCREASE EXCITATORY INNERVATION UPON HIGH

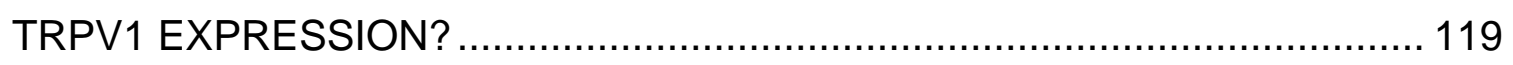

4.9.1. BDNF controls excitatory presynaptic strength but not excitatory innervation to TRPV1 transfected neurons............................................... 119

4.9.2. BDNF does not regulate excitatory or inhibitory innervation in OLM

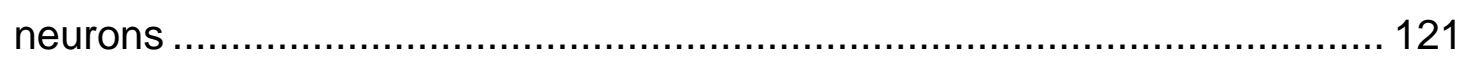

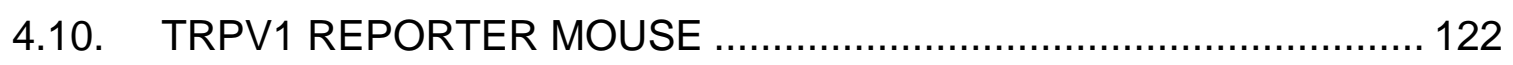

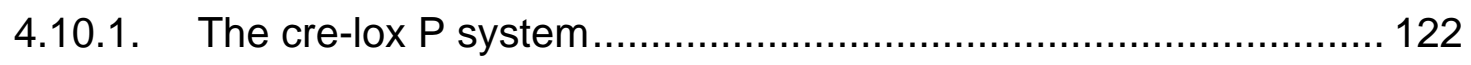

4.10.2. The TRPV1-tdTomato reporter mouse line................................ 124

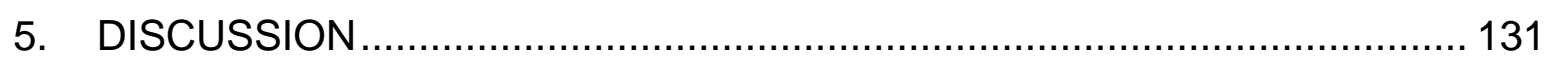

5.1. TRPV1 EXPRESSION IN THE HIPPOCAMPUS .............................. 131

5.1.1. TRPV1 and VR.5' sv are differentially expressed in the mouse DRG

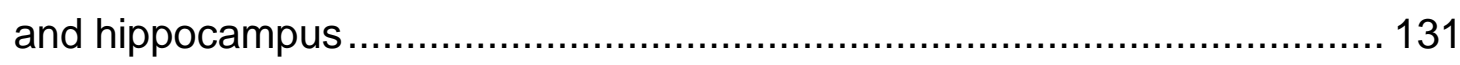

5.1.2. Quantification of TRPV1 expression during development............... 132

5.1.3. Remaining expression of the TRPV1 C-T in TRPV1 KOs............... 133

5.1.4. TRPV1 is expressed functionally in a subset of OLM inhibitory

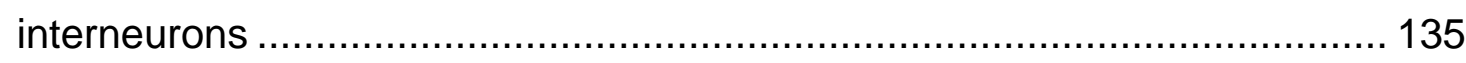

5.1.5. TRPV1-expressing OLM neurons: NGF and BDNF ..................... 138

5.1.6. Expression of functional TRPV1 in hippocampal neurons .............. 140

5.1.7. TRPV1 expression and activation regulate excitatory synaptic innervation in OLM neurons ................................................................. 140 


\section{TABLE OF CONTENTS}

5.1.8. Possible TRPV1 endogenous ligands and binding partners in the

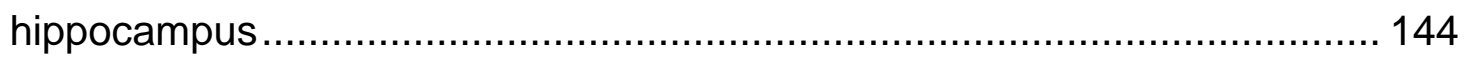

5.1.9. Regulation of TRPV1 expression in hippocampal neurons: link to

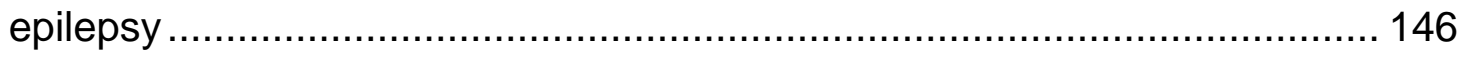

5.1.10. TRPV1 affects synaptic plasticity in the hippoocampus............... 149

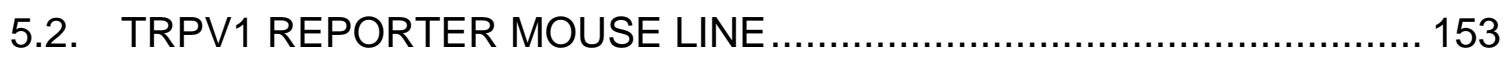

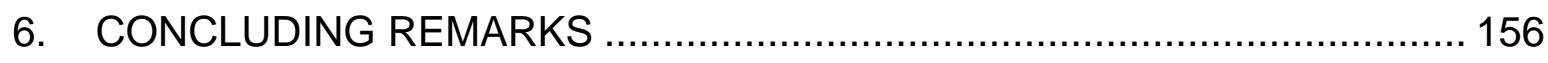

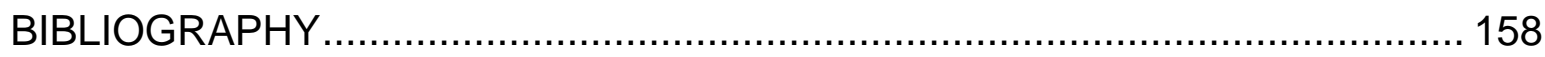




\section{TABLE OF FIGURES}

Figure 1. Diagram of the putative structure of TRPV1 and VR.5' sv .................. 34

Figure 2. Diagram of a CA1 microcircuit involving OLM neurons. ...................... 45

Figure 3. fEPSP recording protocol ............................................................. 71

Figure 4. Differential mRNA expression of specific TRPV1 domains and VR.5' sv

Figure 5. Differential mRNA expression of specific TRPV1 domains and VR.5' sv in the DRG and hippocampus from WT mice . 83

Figure 6. TRPV1 expression in mouse brain and hippocampus ......................... 84

Figure 7. Functional TRPV1 expression in mouse hippocampal neurons in vitro. 87 Figure 8. Expression of the TRPV1 N-terminal alternative splice isoform VR.5' sv

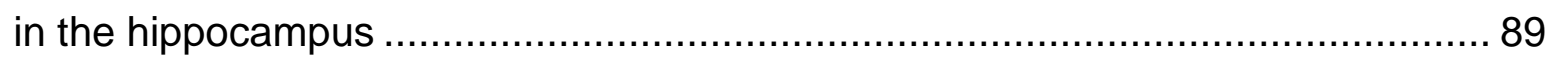

Figure 9. TRPV1 is highly expressed in OLM neurons in the hippocampus ......... 91

Figure 10. High TRPV1 expression in a subset of hippocampal inhibitory interneurons in vitro 93

Figure 11. TRPV1 deficient mice have a normal cortical and hippocampal laminar architecture. 95

Figure 12. Increased excitatory innervation of high TRPV1 expressing OLM neurons 97

Figure 13. Post-synaptic TRPV1 promotes excitatory innervation of OLM cells ... 98 Figure 14. Inhibitory innervation of OLM neurons is less affected by post-synaptic

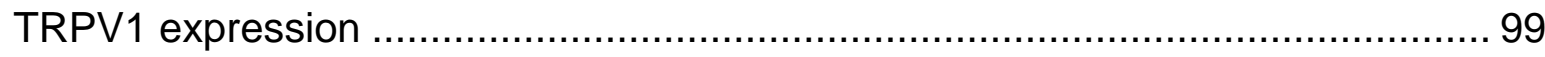

Figure 15. TRPV1 promotes excitatory innervation of OLM neurons................. 101 Figure 16. TRPV1 and TRPV1/VR.5' sv overexpression in neurons increases excitatory innervation 104

Figure 17. TRPV1/VR.5' sv overexpression in neurons decreases inhibitory innervation 105

Figure 18. OLM neurons gate synaptic plasticity in the Schaffer collateral........ 107 


\section{TABLE OF FIGURES}

Figure 19. TRPV1 KOs show reduced Schaffer collateral LTP that is rescued by nicotine treatment 108

Figure 20. NGF promotes expression of TRPV1 in hippocampal neurons

Figure 21. High TRPV1 expression correlates with increased BDNF levels in hippocampal neurons.

Figure 22. TRPV1 expression is increased by neuronal activity in hippocampal neurons

Figure 23. TRPV1 expression is increased by neuronal activity in hippocampal neurons

Figure 24. TRPV1 overexpression in neurons increases excitatory innervation through a BDNF-independent mechanism

Figure 25. BDNF is not required for increased excitatory and inhibitory innervation of high TRPV1 expressing neurons.

Figure 26. tdTomato expression pattern in brain in the TRPV1cre X Rosa26-

tdTomato reporter mouse line. 126

Figure 27. Hippocampal expression pattern of tdTomato signal in the TRPV1cre $X$ Rosa26-tdTomato reporter mouse line. 127

Figure 28. Differences in the tdTomato expression pattern in the hippocampus of the $\mathrm{F} 1$ and $\mathrm{F} 2$ generation 129 


\section{ABBREVIATIONS}

${ }^{\circ} \mathrm{C} \quad$ Degrees Celsius (centigrade)

$\mu \mathrm{g} \quad$ Microgram

$\mu \mathrm{g} / \mathrm{ml} \quad$ Micrograms per milliliter

$\mu \mathrm{l} \quad$ Microliter

ACSF Artificial cerebrospinal fluid

AP-5 (2R)-amino-5-phosphonovaleric acid

au Arbitrary units

BCA Bicinchoninic acid assay

BDNF Brain-derived neurotrophic factor

bp Base pairs

CA1-3 Cornu ammonis 1-3

cDNA Complementary DNA

c-Fos Fos proto-oncogene-proteins

$\mathrm{cm} \quad$ Centimeter

cm2 Square centimeter

CNS Central nervous system

Cre Cre recombinase

C-T C-terminus

DAPI $\quad 4^{\prime}, 6$-Diamidin-2-phenylindol

ddH2O Double destilled water

DIV Day in vitro

DNA Deoxyribonucleic acid

dNTPs Deoxynucleotides

DPSSL Diode-pumped solid-state lasers

EDTA Ethylenediaminetetraacetic acid

EGFP Enhanced green fluorescent protein

ENI European Neuroscience Institute 


$\begin{array}{ll}\text { ABBREVIATIONS } \\ \text { ENI } & \text { European Neuroscience Institute } \\ \text { fEPSP } & \text { field excitatory post synaptic potential } \\ \text { g } & \text { G-Force } \\ \text { GABA } & \text { Y-aminobutyric acid } \\ \text { GAD65 } & \text { Glutamic acid decarboxylase 65 } \\ \text { HBSS } & \text { Hank's balanced salt solution } \\ \text { HEK } & \text { Human embryonic kidney } \\ \text { HFS } & \text { High frequency stimulation } \\ \text { hr } & \text { Hour(s) } \\ \text { HRP } & \text { Horseradish peroxidase } \\ \text { ICC } & \text { Immunocytochemistry } \\ \text { IHC } & \text { Immunohistochemistry } \\ \text { kD } & \text { Kilodalton } \\ \text { kHz } & \text { Kilohertz } \\ \text { KO } & \text { Knock out } \\ \text { I } & \text { Liter } \\ \text { LB } & \text { Lysogenic broth } \\ \text { LFS } & \text { Low frequency stimulation } \\ \text { LTD } & \text { Long term depression } \\ \text { LTP } & \text { Long term potentiation } \\ \text { M } & \text { Molar (gram/mol) } \\ \text { MAP2 } & \text { Microtubule-associated protein 2 } \\ \text { mEPSC } & \text { Miniature excitatory postsynaptic current } \\ \text { mGluR7 } & \text { Metabotropic glutamate receptor } 7 \\ \text { min } & \text { Minute(s) } \\ \text { mIPSC } & \text { Miniature inhibitory postsynaptic current } \\ \text { mI } & \text { Milliliter } \\ \text { Millimeter } \\ \text { Millimolar } \\ \text { milliosmole } \\ \text { Millivolt }\end{array}$




$\begin{array}{ll}\text { ABBREVIATIONS } \\ \text { MS } & \text { Megaohm } \\ \text { NB } & \text { Neurobasal medium } \\ \text { NB+ } & \text { Culture medium } \\ \text { NBQX } & \text { 2,3-dihydroxy-6-nitro-7-sulfamoyl-benzo[f]quinoxaline-2,3-dione } \\ \text { NGF } & \text { Nerve growth factor } \\ \text { nm } & \text { Nanometer } \\ \text { N-T } & \text { N-terminus } \\ \text { OD600 } & \text { Optical density of 600 } \\ \text { OLM } & \text { Oriens lacunosum moleculare } \\ \text { Osm } & \text { Osmole } \\ \text { P0 } & \text { Postnatal day 0 } \\ \text { pA } & \text { Picoampere } \\ \text { PAGE } & \text { Polyacrylamide gel electrophorese } \\ \text { PB } & \text { Phosphate buffer } \\ \text { PBS } & \text { Phosphate-buffered saline } \\ \text { PCR } & \text { Polymerase chain reaction } \\ \text { PDL } & \text { Poly-D-Lysine } \\ \text { PFA } & \text { Paraformaldehyde } \\ \text { PNS } & \text { Peripheral nervous system } \\ \text { PV25 } & \text { Pavalbumin 25 } \\ \text { qPCR } & \text { Quantitative PCR } \\ \text { RFP } & \text { Red fluorescent protein } \\ \text { RNA } & \text { Ribonucleic acid } \\ \text { RT } & \text { Room temperature } \\ \text { SB } & \text { N-(3-Methoxyphenyl)-4-chlorocinnamide (SB-366791) } \\ \text { SDS } & \text { Sodium dodecyl sulfate } \\ \text { SEM } & \text { Standard error mean } \\ \text { SMI-312 } & \text { Neurofilament marker } \\ \text { SO } & \text { Stratum oriens } \\ \text { Syt1 } & \text { Synaptotagmin 1 } \\ \text { Syt1u } & \text { Synaptotagmin 1 lumenal domain antibody uptake } \\ & \end{array}$




$\begin{array}{ll}\text { ABBREVIATIONS } \\ \text { Taq } & \text { Thermus aquaticus } \\ \text { TB } & \text { Tris buffer } \\ \text { TBS } & \text { Tris buffer saline } \\ \text { TBST } & \text { Tris buffer saline with triton } \\ \text { TrkB } & \text { Tropomyosin receptor kinase B } \\ \text { TRPV1 } & \text { Transient receptor potential cation channel subfamily V member 1 } \\ \text { TRPV2 } & \text { Transient receptor potential cation channel subfamily V member 2 } \\ \text { TRPV4 } & \text { Transient receptor potential cation channel subfamily V member 4 } \\ \text { TTX } & \text { Tetrodotoxin } \\ \text { U/ml } & \text { Units per milliliter } \\ \text { UMG } & \text { Universität Medizin Göttingen } \\ \text { UV } & \text { Ultraviolet } \\ \text { v/v } & \text { Volume per volume } \\ \text { vGAT } & \text { Vesicular GABA transporter } \\ \text { vGlut1 } & \text { Vesicular glutamate transporter 1 } \\ \text { VIP } & \text { Vasoactive Intestinal peptide } \\ \text { VR.5' sv } & \text { Vanilloid receptor 5'-splice variant } \\ \text { w/v } & \text { Weight per volume } \\ \text { WB } & \text { Western blot(s) } \\ \text { WT } & \text { Wild type } \\ \text { SC } & \text { Schaffer collateral } \\ \text { TET } & \text { Tetanic stimulation }\end{array}$




\section{ABSTRACT}

The transient receptor potential vanilloid channel 1 (TRPV1) is a non-selective ion channel capable of being activated by multiple factors such as noxious stimuli, heat $\left(>42{ }^{\circ} \mathrm{C}\right.$ ), low $\mathrm{pH}$ and pungent agents. Due to its pivotal role in pain perception TRPV1 has extensively been studied in the context of nociception in sensory neurons. Although TRPV1 has been discovered in the hippocampus, the exact cellular and subcellular expression as well as the function of the channel is still subject of strong debate. Using multiple techniques our study confirms the functional expression of TRPV1 in hippocampal neurons and provides information about the localization of the receptor. We found that the expression of TRPV1 was upregulated by NGF and high neuronal activity. TRPV1 was highly expressed in reelin/somatostatin-positive interneurons of the oriens lacunosum moleculare (OLM) that concomitantly express NGF (that likely increases TRPV1 expression). TRPV1 promotes high excitatory innervation of OLM neurons; when activated the channel recruits more excitatory terminals but if the channel is blocked or removed OLM neurons receive substantially less excitatory input. Such impairment is reflected in a significant decrease in LTP in the Schaffer collateral pathway in the TRPV1 KO mouse. This deficit in LTP is bypassed when OLM neurons are activated with nicotine - which selectively activates only OLM neurons in the hippocampus. This demonstrates functionally that TRPV1 is specifically expressed in OLM neurons. We also report some drawbacks detected in the TRPV1 knockout and the TRPV1 cre/lox $P$ reporter mouse, two commercially available mouse lines commonly used in the field. Our findings may encourage other researchers to be more cautious with conclusions drawn using these animals. Together this thesis sheds light on the role of TRPV1 in synaptic plasticity in the hippocampus. 


\section{INTRODUCTION}

The transient receptor potential (TRP) channel family comprises a wide variety of ionic channels that are involved in multiple neurosensory mechanisms (e.g, photo, osmotic, mechanical, thermal, acidic and pain perception) (Bevan et al., 2014; Caterina et al., 1997; Clapham, 2003; Liedtke and Kim, 2005; O'Neil and Heller, 2005; Patapoutian, 2005; Pedersen et al., 2005; Tominaga and Caterina, 2004). This family has been found in almost all phyla, being absent only in plants and bacteria (Nilius and Owsianik, 2011; Vriens et al., 2009). Traditionally, this family has been divided into six subfamilies: TRPC (canonical) TRPA (ankyrin), TRPM (melastatin), TRPV (vanilloid), TRPML (mucolipin) and TRPP (polycystin) (Clapham, 2003; Nilius and Owsianik, 2011; Vriens et al., 2009; Wu et al., 2010). Within the transient receptor potential vanilloid subfamily six different channels have been characterized (TRPV1-6) (Caterina, 2007; Caterina et al., 1997, 1999; Clapham, 2003; Smith et al., 2002). TRPV1 is by far the most investigated member of the vanilloid subfamily and perhaps of the entire TRP family.

The TRPV1 channel is a non-selective, ligand-gated cation channel permeable to monovalent, divalent as well as to polyvalent cations such as $\mathrm{Na}^{+}, \mathrm{Fe}^{+}, \mathrm{K}^{+}, \mathrm{Cs}^{+}, \mathrm{Li}^{+}$, $\mathrm{Rb}^{+}, \mathrm{Ca}^{2+}, \mathrm{Mg}^{2+}$, guanidinium, $\mathrm{N}$-methyl-D-glucamine and tetraethylammonium (Bevan et al., 2014; Caterina et al., 1997; Cesare and McNaughton, 1996; Hellwig et al., 2004; Kauer and Gibson, 2009; Oh et al., 1996; Samways and Egan, 2011; Winter et al., 1990); polyamines such as putrescine, spermine and spermidine have also been reported to be also permeate through TRPV1 channels (Ahern et al., 2006). Permeability to larger cations has been suggested to occur during pore dilatation upon sustained activation of the channel (Bevan et al., 2014; Chung et al., 2008). 


\section{INTRODUCTION}

Perhaps the most striking outstanding fact about TRP channels is their polymodality, that is, the capability of coping with a wide variety of endogenous and exogenous stimuli. As explained in section 2.3 TRPV1 activation or inhibition is gated and regulated by thermal, protonic and voltaic energy, by pungent molecules (e.g. capsaicin), enzymes (e.g. PKA and PKC), endocannabinoids, phospholipids and many more molecules. How does TRPV1 integrate all of these signals in different tissues? Which physiological phenotypes are affected by the signaling of TRPV1? These are central questions of high relevance to medical science and neuroscience.

\subsection{TRPV1 GENE TRANSCRIPTION AND PROTEIN STRUCTURE}

\subsubsection{The Trpv1 Gene}

The trpv1 gene is located in Homo sapiens on chromosome 17 p3, in Mus musculus on chromosome $1145.25 \mathrm{cM}$ and in Rattus norvegicus on chromosome 10 q24. The gene consists of 19 exons in humans, 17 exons in the house mouse, and 16 exons in the Norway rat. In humans and the house mouse, the resulting protein is predicted to be 839 amino acids long, and in Norway rats it is 838 amino acids. The approximate molecular mass of the full length TRPV1 is $95,000 \mathrm{Da}$ (Birnbaumer et al., 2003; Caterina et al., 1997, 2000).

\subsubsection{Trpv1 transcription}

The mechanism of TRPV1 transcription is still unclear. It is known that during inflammation and hyperalgesia the expression of growth factors, such as NGF as well as other inflammatory agents is increased in nociceptors, subsequently upregulating TRPV1 mRNA and protein expression (Amaya et al., 2003, 2004; Ji et 


\section{INTRODUCTION}

al., 2002; Malin et al., 2006) (see section 2.7.1). TRPV1 transcription is prompted by the activation of two putative promoters (located either distally or proximally to the trpv1 gene sequence) in a 2826 bp sequence upstream of the TRPV1 gene. Activation of a GC box enriched sequence located on the more distal upstream regulatory element, by $\mathrm{Sp} 1 / \mathrm{Sp} 4$ transcription factors is essential for NGF-mediated TRPV1 upregulation (Chu et al., 2011; Xue et al., 2007). Alternatively, binding of Runx 1 and CEBP/ $\beta$ transcription factors (separately or cooperatively) to nucleotidic sequences present in a 480 bp-long promoter, overlapping with the distal regulatory element previously described, also induces trpv1 gene expression (Ugarte et al., 2013). These studies describe alternative regulatory cascades by which TRPV1 expression can be regulated. As mentioned in section 2.7.1, TRPV1 expression can be modulated through multiple pathways, thus is likely that the interplay of additional transcription factors and regulatory elements could maintain and modulate TRPV1 expression.

\subsection{TRPV1 STRUCTURE}

The topology of TRPV1 (as all TRP channels) includes three main components:

1) the intracellular amino ( $\mathrm{N}-$ ) and carboxyl (C-) termini, 2) six transmembrane domain helices (TM 1-6) and 3) a pore region (loop) consisting of a pore "turret", a pore helix and an ion selectivity domain ((Bevan et al., 2014; Latorre et al., 2009; Liao et al., 2013; Moiseenkova-Bell et al., 2008; Nagy et al., 2014; Salazar et al., 2009). The TRPV1 channels are known to form tetrameric ion channel complexes with other proteins or subunits. Usually TRPV1 is expressed as self-associating homotetramers (Kedei et al., 2001), however TRPV1 can also form heterotetrameric structures with splice isoforms and other TRP channels like TRPA1, TRPV2 and TRPV3 (Nagy et al., 2014; Rutter et al., 2005; Schumacher and Eilers, 2010; Schumacher et al., 2000; Smith et al., 2002). 


\section{INTRODUCTION}

Akin to other TRPV channels, the N-terminus of TRPV1 possesses an ankyrin domain of six repeats. This domain is essential for protein-protein interactions and channel assembly ( $\mathrm{Li}$ et al., 2006; Liao et al., 2013; Lishko et al., 2007); for instance, calmodulin and ATP binding to specific ankyrin regions leads to $\mathrm{Ca}^{2+}$ mediated sensitization (Hung et al., 1998; Koplas et al., 1997; Lishko et al., 2007). The Src-kinase-dependent phosphorylation of the Y199 ankyrin residue (upon NGF stimulation) has been implicated in TRPV1 insertion in the plasma membrane (Zhang et al., 2005). Besides the ankyrin repeats, other $\mathrm{N}$-terminal residues influence TRPV1 dynamics. For instance, the residue H378 has been implicated in the activation of TRPV1 upon intracellular alkalinization (Dhaka et al., 2009). Also, as mentioned in section 2.3.3, phosphorylation sites for PKA and dephosphorylation sites for calcineurin in the $\mathrm{N}$-terminus are needed for sensitization and desensitization, respectively (Bhave et al., 2002; Jeske et al., 2006; Mohapatra and $\mathrm{Nau}, 2005)$.

One of the most fundamental structural components of the TRPV1 channel is the TRP box present in the C-terminus; a hydrophobic amino acid stretch that is conserved in TRPV, TRPM and TRPC channel subfamilies (Montell, 2001). The TRP domain is crucial for TRPV1 channel homotetramerization (García-Sanz et al., 2004) and contributes to channel gating by integrating stimuli and downstream signals that ultimately lead to the opening of the aqueous channel pore (GarcíaSanz et al., 2007). Specifically, residues 1696, W697 and R701 define the gating energy upon TRPV1 channel activation with capsaicin, heat and voltage (Valente et al., 2008). Besides the TRP domain, specific regions of the C-terminus have been implicated in TRPV1 affinity for ATP and the gating of the channel in response to low $\mathrm{pH}$, heat and voltage; other residues give TRPV1 its specific thermal sensitivity (Brauchi et al., 2006; Kwak et al., 2000; Vlachová et al., 2003). Phosphorylation at the C-terminus of TRPV1 is mainly mediated by PKC (Bhave et al., 2003; Nagy et al., 2014; Numazaki et al., 2002), although phosphorylation by PKA has been reported to occur at certain motifs in the C-terminus (Mohapatra and Nau, 2003). Interestingly PI(4,5)P2 and calmodulin also bind to motifs of the C- 


\section{INTRODUCTION}

terminus but their possible role in either TRPV1 channel sensitization or desensitization, remains unclear (Brauchi et al., 2007; Numazaki et al., 2003).

The most fundamental structure of the TRPV1 channel, the pore region, lies between TM 5 and 6 . The amino acidic composition of the pore loop has a major impact in the selectivity and transport of ions through the pore. The pore itself has been calculated to be $10.1 \AA$ in diameter (Chung et al., 2008) and residue D646N has been hypothesized to form a negatively charged ring that would allow cationic permeation through the aqueous pore. Two intracellularly located amino acid sequences in the pore domain restrict the passage of ca. $9 \AA$ and ca. $6 \AA$ molecules, where residue 1679 is at the tightest point (5.3 $\AA$ ) of the permeation filter (Liao et al., 2013). This residue also aids channel gating upon capsaicin and heat stimulation (Cao et al., 2013; Salazar et al., 2009). The pore turret partially confers thermal activation through a conformational rearrangement that allows gating of the channel (Yang et al., 2010). On the extracellular side of the pore region some residues show affinity for proton-binding in conditions of low pH (Aneiros et al., 2011; Jordt et al., 2000). TRPV1 activation by nitric oxide has been less studied but it seems that cysteine s-nitrosylation of two proximal pore domains causes nitric oxide evoked channel gating (Yoshida et al., 2006).

Another important regulatory domain of the TRPV1 channel is the vanilloid domain located within TM 1-4, which is thought to be the most important binding region for endogenous and exogenous vanilloids (Nagy et al., 2014). Intracellularly, anandamide and capsaicin - that has passed into cells through the plasma membrane - bind to the Y511 residue; the vanillyl site of capsaicin specifically binds Y511, whereas the hydrophobic portion of capsaicin binds to T550 (Gavva et al., 2004; Jordt and Julius, 2002). Resiniferatoxin also specifically binds via its vanillyl domain to at least the L547M residue, and via its orthophenyl group to Y511 (Gavva et al., 2004; Lee et al., 2011). The vanilloid domain, in part, is also responsible for TRPV1 sensitivity to low pH (Jordt and Julius, 2002); together with the pore residue T633, the residue $V 538$, located between TM 3 and 4, transduces 


\section{INTRODUCTION}

channel activation by protonation into gating (Ryu et al., 2007). Additionally, the vanilloid domain bears various phosphorylation sites. For instance, residue S502 undergoes PKA-dependent phosphorylation to sensitize channels to heat (Rathee et al., 2002), whereas phosphorylation by PKC sensitizes TRPV1 channels by intensifying capsaicin responses (Numazaki et al., 2002).

\subsection{TRPV1 LIGANDS}

Substantial research over the last two decades has been dedicated to understand the molecular mechanism of TRPV1 channel activity and its dynamics upon direct interaction with exogenous and endogenous ligands. The tremendous multimodal nature of TRPV1 confers a high complexity to the molecular cascades underlying activation, blockade, sensitization, desensitization and tachyphylaxis of the channel's activity.

Recently two models have been proposed to explain the multimodal signaling integration of the TRPV1 receptor. The first model describes a sequential mechanism of channel activation from an "inactive" to a more dynamic state, predicting that every stimulation of the channel would shift its sensitivity to voltage to a more functional state (Nilius and Voets, 2005). A second allosteric model postulates that specific sensors for different stimulants are each independently linked to the gating system, thus allowing different stimuli to separately activate TRPV1 (Latorre et al., 2007).

\subsubsection{Agonists}

Sensing of thermal and acidic external cues must be indispensable for an organism to survive. It is likely that such cellular receptors would be perceptive to a multitude of environmental cues. TRPV1 is a promiscuous receptor, binding to a wide variety of exogenous and endogenous stimuli and molecules. The TRPV1 channel is 


\section{INTRODUCTION}

known to be activated by various exogenous stimuli such as heat $\left(>43^{\circ} \mathrm{C}\right)$, noxious stimuli, acidic conditions $(\mathrm{pH}<5.9)$, natural pungent agents such as trans-8-methylNvanillyl-6-nonenamide (capsaicin) found in fruits of many Capsicum plants (Thresh, 1876), and other plant and animal toxins such as resiniferatoxin (Caterina et al., 1997, 2000; Holzer, 1991; Jancsó-Gábor et al., 1970a; Kauer and Gibson, 2009; Szolcsányi, 1977; Tominaga et al., 1998; Vriens et al., 2008). Interestingly the TRPV1 channel is also gated by endogenous agonists both in the PNS and CNS. These endogenous activators have been referred to as endovanilloids (Bevan et al., 2014; Kauer and Gibson, 2009; Martins et al., 2014; van der Stelt and Di Marzo, 2004; Vriens et al., 2009). Arachidonylethanolamide, also known as anandamide, was the first endogenous ligand to be discovered to activate TRPV1 (Tóth et al., 2009; Zygmunt et al., 1999). Although one can refer to anandamide as an endovanilloid, it was originally described as an endocannabinoid, since it was first discovered to bind to the cannabinoid receptor 1 (Devane et al., 1992). After this discovery, the list of "endovanilloids" found to activate TRPV1 especially in the PNS has been continuously growing. Endogenous activators include endocannabinoids (e.g. NADA), ethanolamides (e.g. anandamide and $\mathrm{N}$ oleoylethanolamide) oxygenated eicosatetraenoic acids, lypogenase products, several endogenous phospholipids (e.g. lysophosphatidic acid), oxidation products, prostaglandins, adenosine, ATP, polyamines, protons and inflammatory agents (Ahern, 2003; Bevan et al., 2014; Huang et al., 2002; Nieto-Posadas et al., 2012; Vriens et al., 2009). Initially TRPV1 sensitivity to voltaic changes was not observed (Caterina et al., 1997). Subsequently, another study suggested that the channel's C-T motif could contribute to changes in voltage sensitivity upon plasma membrane depolarization (Vlachová et al., 2003). Regulation of TRPV1 due to changes in voltage, as during neuronal depolarization, could in principle lead to dramatic changes in the activity of the receptor.

Most of the identified TRPV1 activators were discovered in the PNS (where most of the research has been developed). In the CNS less is known about the factors that could modulate TRPV1 activity. Most of the research has been focused on if the 


\section{INTRODUCTION}

molecules that regulate TRPV1 activity in the PNS also control it in the CNS. 12S-, 15S- and 5S-HPETE, anandamide, 2-arachidonylglicerol (2-AG), Noleoylethanolamide, and $\mathrm{N}$-arachidonoyldopamine (NADA) are examples of endovanilloids that activate TRPV1 in the PNS and have been found to be expressed in the CNS (including the hippocampus); anandamide and NADA are more abundant in the CNS than in the PNS (Ahern, 2003; Goswami et al., 2010; Huang et al., 2002; Hwang et al., 2000; Martins et al., 2014; Movahed et al., 2005; Musella et al., 2010; van der Stelt and Di Marzo, 2004; Zygmunt et al., 1999, 2013). Interestingly, the endovanilloids mentioned above (also categorized as endocannabinoids) are capable of activating both CB1 and TRPV1 suggesting a narrow interplay between these two systems thus postulating TRPV1 as a polymodal receptor integrating copious amounts of neurosensory information (Chávez et al., 2010; Grueter et al., 2010; Marzo, 2010; Marzo and Petrocellis, 2012).

Many other factors have been found to gate and regulate TRPV1 channel activity leading to different changes on the conformational state of the channel some of them include bradykinin, NGF, histamine, adenosine, spermine and ATP, which are also found in the CNS, thus become feasible candidates to regulate TRPV1 channel activity in the brain (Kauer and Gibson, 2009; Shu and Mendell, 1999; Szallasi et al., 2007).

\subsubsection{Antagonists}

Compared to the immense number of reported TRPV1 agonists, fewer antagonists have been discovered. Perhaps the most commonly known and applied TRPV1 antagonists are ruthenium red and capsazepine (a TRPV1 competitive blocker) (Caterina et al., 1997; Gunthorpe et al., 2004; Vriens et al., 2009; Walpole et al., 1994). Despite their extensive use in traditional pharmacological studies, ruthenium red and capsazepine have some disadvantages, compared to recently synthesized antagonists. Ruthenium red is a non-competitive, non-selective TRPV1 antagonist 


\section{INTRODUCTION}

(Dray et al., 1990; García-Martínez et al., 2000) that has been found to inhibit other TRP and TRPV channels such as TRPV 3 and TRPV4 which are known to interact or dimerize with TRPV1 (Brown et al., 2013; Gunthorpe et al., 2004; Hoenderop et al., 2001; Nilius et al., 2001; Smith et al., 2002; Vincent and A.J. Duncton, 2011; Vriens et al., 2009). Capsazepine, on the other hand, has reduced metabolic stability and diverse pharmacokinetic properties in different species (Walker et al., 2003). Due to the medical relevance of a TRPV1 specific antagonist for treatment of pain, in recent years a wide variety of TRPV1-specific antagonist have been synthesized and catalogues have been developed to guide research due to the heterogeneity of the mechanism of action of different inhibitors on TRPV1 (Szallasi et al., 2007; Vriens et al., 2009). In our study we decided to use a small molecule discovered to be a potent TRPV1-selective antagonist, called SB-366791, which was proven to block the effects of capsaicin on TRPV1 activity and to cause more efficient inhibition than capsazepine (Gunthorpe et al., 2004; Varga et al., 2005).

Recently some molecules that are endogenously expressed have also been found to block the gating of TRPV1, thus reducing the receptor's activity. For instance: cholesterol enrichment decreases capsaicin and heat evoked-responses in TRPV1-transfected HEK293 cells (Picazo-Juárez et al., 2011). Sphingomyelinase and resolvins (omega 3 derivates) are two more molecules recently suggested to inactivate TRPV1 channels or at least to inhibit TRPV1 activation in sensory neurons (Park et al., 2011a; Sántha et al., 2010). Adenosine has also been proposed to act as a competitive antagonist of TRPV1, by directly binding to the channel leading to a blockade of capsaicin-mediated TRPV1 responses (Puntambekar et al., 2004).

\subsubsection{Modulators}

TRPV1 is not only activated or directly regulated by ligands; multiple factors such as protein kinases, inflammatory agents, calcineurin, calmodulin and phosphoinositides modulate channel activity by interacting with motifs on the C- 


\section{INTRODUCTION}

and $\mathrm{N}$-termini leading either to sensitization through channel phosphorylation or to $\mathrm{Ca}^{2+}$-dependent desensitization, mainly via de-phosphorylation (Bevan et al., 2014; Bhave et al., 2002, 2003; Docherty et al., 1996; Kim et al., 2008; Lishko et al., 2007; Malin et al., 2006; Shu and Mendell, 1999).

Sensitization of TRPV1, which makes the channel more susceptible to its activators (e.g. heat and capsaicin), is driven by the binding of protein kinases $A$ and $C$ and other inflammatory agents (e.g. bradykinin, NGF and GDNF) directly to TRPV1 (Amadesi et al., 2006; Bhave et al., 2003; Malin et al., 2006; Mohapatra and Nau, 2003; Shu and Mendell, 1999; Sugiura et al., 2002).

Conversely, TRPV1 is known to be desensitized (have a decreased sensitivity to activators) by constant or repetitive channel activation, which causes a downregulation in channel activity (Liu and Simon, 1996; Novakova-Tousova et al., 2007). Desensitization of TRPV1 is most likely achieved by multiple mechanisms, either by activation of calcineurin after $\mathrm{Ca}^{2+}$ influx upon TRPV1 channel opening (Docherty et al., 1996; Mohapatra and Nau, 2005), phospholipase-C (PLC)dependent PI(4,5)P2 hydrolysis (Jeske et al., 2011; Kim et al., 2008) or by a direct binding of calmodulin to TRPV1 (Lau et al., 2012; Lishko et al., 2007; Numazaki et al., 2003).

In summary TRPV1 channels regulate a multitude of signaling cascades in response to various activators and modulators. Still much of the knowledge acquired for this receptor has been obtained from studies in the peripheral nervous system. Since $\mathrm{pH}$ and temperature are mostly at "normal" physiological levels in higher neuronal regions (i.e. the brain), the question of the functionality of the channel in these areas arises. The precise role of TRPV1 in the CNS and especially in the hippocampus remains unclear. 


\section{INTRODUCTION}

\subsection{TRPV1 EXPRESSION}

Although TRPV1 expression has been reported in various neuronal and nonneuronal tissues including the skin (cutaneous sensory nerve fibers, mast cells, epidermal keranocytes, dermal blood vessels, differentiated sebocytes and sweat glands) pancreas (B-cells), bladder (urothelium, smooth muscle, blood vessels and neurons), blood (mononuclear cells and synoviocytes), adipose tissue, vascular smooth muscle, arteriolar smooth muscle cells, mesenteric arteries, endothelial cells, pulmonary artery smooth muscle, laryngeal epithelium, nasal vascular endothelium, epithelials and submucosal glands (reviewed in Fernandes et al., 2012; Nilius and Owsianik, 2011), TRPV1 expression is best characterized in neuronal cell types of the PNS.

TRPV1 was first reported and characterized in the PNS; specifically in the dorsal root, vagal and trigeminal ganglia, where it was found to be expressed in small and medium-sized afferent $A \delta$ and $C$-fibers of peptidergic nociceptive neurons, which in turn project to innervate nearly all organs and tissues (Bevan et al., 2014; Caterina et al., 1997; Holzer, 1991; Jancsó et al., 1977; Tominaga et al., 1998). In the DRG, TRPV1 was originally detected in two groups of neurons; one responding to NGF and positive for CGRP and substance $P$, and another expressing the glial derived nerve factor (GDNF) (Tominaga et al., 1998). It is well-established that NGF has a major role in sustaining the expression of TRPV1 in nociceptors. Under certain conditions such as during inflammation, increased expression of NGF upregulates the expression of TRPV1 (Bevan and Winter, 1995; Ji et al., 2002) (see section 2.7.1)

The original study that identified the TRPV1 receptor to be activated by noxious heat and capsaicin (see section 2.3.1), failed to detect TRPV1 expression in brain and spinal cord (Caterina et al., 1997). However earlier studies had already found that in the hypothalamus, namely in the pre-optic area, capsaicin injection induced 


\section{INTRODUCTION}

hypothermia in rats (through TRPV1-dependent desensitization) (Gavva, 2008; Jancsó-Gábor et al., 1970a). Later studies confirmed the expression of TRPV1 in the hypothalamus and its pivotal role for the maintenance of body temperature (Cavanaugh et al., 2011; Gavva, 2008; Roberts et al., 2004; Sharif-Naeini et al., 2008). Due to the heterogeneity of information regarding the expression of TRPV1 in the CNS there has been a constant disagreement in the scientific community regarding whether TRPV1 functions in the brain. One problem could be that TRPV1 has been reported to be expressed in lower levels in the brain (Han et al., 2012; Sanchez et al., 2001; Szallasi et al., 1995) than in nerves from the PNS, which can lead to misinterpretation, depending on technique employed (Kauer and Gibson, 2009; Martins et al., 2014). Yet the list of CNS and brain regions where TRPV1 expression has been detected has been increasing. Through a wide variety of methodologies, TRPV1 has been identified in many areas of the CNS including the spinal cord, hypothalamus, thalamic nuclei, amygdala, cerebellum, telencephalon (entorhinal cortex, olfactory nuclei as well as in the frontal, piriform, parietal and retrosplenial cortices), midbrain (periaqueductal gray, the interpeduncular and raphe nuclei, and substantia nigra), hindbrain (superior colliculus, locus coeruleus, vagus dorsal motor nucleus and nucleus of the solitary tract), septal regions and the hippocampus, where some studies have confirmed a broad expression of the channel in the brain (Cavanaugh et al., 2011; Cristino et al., 2006a; Han et al., 2012; Mezey et al., 2000; Roberts et al., 2004; Sanchez et al., 2001; Sasamura et al., 1998; Tóth et al., 2005).

Most of the studies suggesting the presence of TRPV1 in hippocampal neurons have only provided functional evidence of the channel's activity in synaptic plasticity in synaptic transmission by neuropharmacologically modulating TRPV1 channel activity; only a few studies have addressed the cellular and subcellular localization of TRPV1 in the hippocampus (see section 2.6). 


\subsection{TRPV1 IN THE BRAIN: PHYSIOLOGICAL RELEVANCE}

In the PNS, TRPV1 has been extensively studied due to its medical relevance treatment of pain and inflammation. In the CNS, TRPV1 has also been found to be important in physiological processes such as pain, thermoregulation and synaptic plasticity.

\subsubsection{TRPV1 in nociceptive brain circuits}

The involvement of TRPV1 in peripheral nociceptive circuits has long been known. Later studies show that TRPV1 is expressed in brain areas known to participate in the nociceptive system, such as the periaqueductal gray, locus coeruleus, rostral ventromedial medulla, anterior cingulate nucleus, and medial prefrontal cortex (Calejesan et al., 2000; Cristino et al., 2006a; Marinelli et al., 2002; McGaraughty et al., 2003; Mezey et al., 2000). TRPV1 was found to be expressed in glutamatergic neurons in the ventrolateral periaqueductal gray, where activation by capsaicin or anandamide causes anti-nociceptive effects by promoting glutamate release and binding to postsynaptic metabotropic glutamate receptor 1 (mGluR1) at the rostral ventromedial medulla, leading to the activation of "anti-nociceptive neurons" and inhibition of "nociceptive" neurons (Maione et al., 2007; Palazzo et al., 2002). These effects were blocked by the opioid receptor antagonist naloxone, suggesting an interaction of both the opioid and vanilloid mechanisms (Maione et al., 2009). Contrary to this anti-nociceptive effect of TRPV1 activation, glutamate release to the anterior cingulate cortex upon TRPV1 activation has rather been linked to persistent pain induction (Calejesan et al., 2000). More recent studies have suggested that TRPV1 might be involved in other brain areas, for example in morphological changes of the pre- and anterior limbic areas, where TRPV1 blockade stopped such changes and the behavioral responses seen after traumatic neuropathy (Novellis et al., 2011). 


\section{INTRODUCTION}

\subsubsection{Central thermal regulation}

In the early 1970s the role of TRPV1 (still uncharacterized at that time) in the maintenance of body temperature within a physiological range was discovered. In these studies rats and guinea pigs were either generally or locally injected in the hypothalamic preoptic area with capsaicin, which caused hypothermia (JancsóGábor et al., 1970a, 1970b). Later studies showed that injection of TRPV1-specific inhibitors elicits a rise in body temperature (hyperthermia) in different species, including humans (Gavva et al., 2007, 2008). Surprisingly, TRPV1 KO mice do not have impaired maintenance of body temperature (Szelényi et al., 2004; Tóth et al., 2010), but neither TRPV1-specific agonists nor antagonists were able to induce hypo- or hyper-thermia, respectively, in knockout mice; fever responses were also attenuated in TRPV1 KO mice (lida et al., 2005; Tóth et al., 2010). Although the mechanism of action of TRPV1 in maintenance of body temperature is still not fully understood, its role in thermoregulation (in the PNS as well as in the CNS) has been established by many studies from various labs.

\subsubsection{Osmosensing}

Magnocellular neurosecretory cells (MNC) of the supraoptic nucleus of the hypothalamus are known to participate in osmoregulation of the body, mainly through vasopressin (Dreifuss et al., 1971; Sudbury et al., 2010) which is released when MNC are activated during hypertonicity, and ultimately causes basal water reabsorption (thirst) when it interacts with vasopressin 2 receptors in the kidney. On the other hand during hypotonicity MNC activity is inhibited, decreasing vasopressin release that subsequently leads to water excretion (Brimble and Dyball, 1977).

Recently a TRPV1 splice isoform lacking the N-terminus was discovered to be expressed in MNC. Interestingly, capsaicin does not elicit neuronal firing of MNC, 


\section{INTRODUCTION}

reinforcing the notion of a novel capsaicin-insensitive TRPV1 splice variant with unique function in MNC. The depolarization of MNCs during hypertonicity can be stopped by the TRPV1 antagonist ruthenium red (TRPV1 antagonist), and the MNC of TRPV1 KO mice have no cellular shrinking and have reduced vasopressin secretion upon hypertonicity (Naeini et al., 2006; Sharif-Naeini et al., 2008). Notably, TRPV1 has been suggested to be in part controlling thirst, since TRPV1 KO mice show reduced water intake compared to WT animals when confronted with hyperosmotic conditions (Ciura and Bourque, 2006).

\subsubsection{Behavior and emotional states}

TRPV1 has been implicated the in various behavioral and emotional states such as mood, aggression, social interaction, drug addiction and addictive behavior, and depressive-like states such as anxiety and stress (Adamczyk et al., 2012; Ho et al., 2012; Marinelli et al., 2004; Martins et al., 2014; You et al., 2012).

\subsubsection{Anxiety, stress and addiction}

TRPV1 KO mice have reduced anxiety (determined by the elevated plus maze test) and fear conditioning (identified by less "freezing behavior") as well as reduced stress sensitization (Marsch et al., 2007). Some studies have linked TRPV1 activity with the induction of anxiety-related behavior, since intraperitoneally or ventral hippocampal injections with TRPV1 antagonists reduced anxiety and treatment with exogenous agonists induced anxiogenic responses (Hakimizadeh et al., 2012; Kasckow et al., 2004; Santos et al., 2008). TRPV1 activation by injection of anandamide in the prefrontal cortex also elicited anxietyrelated responses, whereas anandamide-dependent CB1 activation and TRPV1 channel blockade rather caused anxiolytic effects (Aguiar, 2009; Rubino et al., 2008). 


\section{INTRODUCTION}

It has been reported that the activity and expression of TRPV1 (sometimes together with the cannabinoid system) is altered in some aspects of metamphetamine-, nicotine-, ethanol- and cocaine-induced addictive behavior (Adamczyk et al., 2012; Grueter et al., 2010; Hayase, 2011; Renteria et al., 2014; Tian et al., 2010). TRPV1 KO animals have a preference for the consumption of ethanol, higher ethanol intake, less sensitization to sedation and faster recovery from ethanol-induced motor incoordination (Blednov and Harris, 2009). In the nucleus accumbens TRPV1 activation counteracts the attenuation in LTD induction caused by ethanol. TRPV1 is known to influence the neuronal "reward" circuit that is involved in addictive behavior and acute stress. This system comprises dopaminergic inputs from the ventral tegmental area (VTA) to both the nucleus accumbens and the prefrontal cortex (Bonci et al., 2003; Chiara and Imperato, 1988; Maeda and Mogenson, 1982; Marinelli et al., 2004; Schultz, 1986). Both capsaicin application in the VTA, and noxious stimuli, enhance dopaminergic secretion onto the nucleus accumbens, which is facilitated by an increase in glutamatergic release onto VTA-dopaminergic neurons (Marinelli et al., 2004). Recently TRPV1 was suggested to engage in the "indirect" reward circuit that induces LTD in medium spiny neurons of the nucleus accumbens (Grueter et al., 2010). In this study, low frequency stimulation and activation of mGluR1 receptors in medium spiny neurons elicited LTD, which subsequently causes a mGluR5dependent anandamide production, where anandamide ultimately binds to presynaptic CB1 (decreasing glutamate release) and postsynaptic TRPV1 receptors (elevating intracellular $\mathrm{Ca}^{2+}$ ). Postsynaptic calcium elevation by anandamide leads to calcineurin-mediated AMPA receptor endocytosis that together with reduced pre-synaptic glutamate release leads to LTD in medium spiny neurons. This form of LTD was absent in animals that consumed cocaine $24 \mathrm{~h}$ prior to the experiment. The exact role of TRPV1 in the clinical symptoms of drug addiction has not been dissected, but TRPV1 has been hypothesized to reinforce cocaine-seeking behavior rather than to cause addiction to the drug itself (Adamczyk et al., 2012). 


\subsection{TRPV1 IN THE HIPPOCAMPUS: EXPRESSION AND ROLE IN SYNAPTIC PLASTICITY}

\subsubsection{TRPV1 expression pattern}

Perhaps one of the most discussed issues in the field of research of TRPV1 is how the expression and possible function of the channel contributes to modifications in synaptic plasticity in the hippocampus (see section 2.6).

As mentioned above, the original study that characterized TRPV1 (and many subsequent studies) did not find TRPV1 expression in hippocampus (Benninger et al., 2008; Caterina et al., 1997; Köfalvi et al., 2006). However, TRPV1 expression at mRNA and protein levels has been detected in the hippocampus using a wide variety of techniques including RT-PCR (for mRNA), ELISA, IHC, in situ hybridization, electron microscopy, western blot, radioactive resiniferatoxin (TRPV1 ligand) for detection of protein levels and more recently, using a TRPV1 reporter mouse line (Bennion et al., 2011; Cavanaugh et al., 2011; Cristino et al., 2006a, 2008; Han et al., 2012; Huang et al., 2014; Merrill et al., 2012; Mezey et al., 2000; Puente et al., 2014; Roberts et al., 2004; Sanchez et al., 2001; Schumacher et al., 2000; Tóth et al., 2005). TRPV1 has been detected in the dentate gyrus (prominently in the molecular layer), and in pyramidal cell somas of the CA1 and CA3 regions of the hippocampus, where it is expressed in ca. 50\% of the CA3 neurons (Cristino et al., 2008; Merrill et al., 2012). Interestingly, TRPV1 was also found in some unidentified interneurons of the stratum oriens (Cristino et al., 2006a, 2008).

Functional assays have found TRPV1 in brain and hippocampus at pre- and postsynapses of both excitatory and (to a lesser extent) inhibitory synapses, and possibly on synaptic vesicles (Cristino et al., 2006a; Goswami et al., 2010; Kauer and Gibson, 2009). Presynaptic TRPV1 activation has been found to influence 


\section{INTRODUCTION}

synaptic transmission by boosting excitatory neurotransmitter release in the substantia nigra (Marinelli et al., 2003; Starowicz et al., 2007) and hypothalamus (Sasamura et al., 1998). On the other hand, in the hippocampus TRPV1 has been related to a novel mechanism of LTD; TRPV1 activation in the pre-synapse decreased glutamate release (Gibson et al., 2008), whereas post-synaptically, its activation caused calcineurin/dynamin-dependent AMPA receptor endocytosis (Chávez et al., 2010). Two studies have shown (using electron microscopy) the post-synaptic localization of TRPV1 both at excitatory and inhibitory synapses in the mouse hippocampal dentate molecular layer (Canduela et al., 2015; Puente et al., 2014) and in CA1 pyramidal neurons at inhibitory interneurons of the stratum radiatum (Lee et al., 2015). The specificity of the TRPV1 antibodies used in these studies, however, was not verified using TRPV1 knockouts. Since most studies to date have addressed the pre- or post-synaptic localization through functional assays, there is still a lack of proof of TRPV1 expression at pre or post-synaptic sites.

The expression of TRPV1 in DRGs during embryogenesis has been reported to start at E13 and gradually increase during later embryonic and postnatal stages, reaching its peak postnatally at $\mathrm{P} 3$, where its expression remains constant at least until P56 (Funakoshi et al., 2006; Hjerling-Leffler et al., 2007). In brain and hippocampus, the postnatal expression of TRPV1, at both mRNA and protein levels, was found in two week old mice and steadily increased, reaching maximum levels 8 weeks after birth; interestingly in DRG, brain and hippocampus TRPV1 expression has been reported to be severely decreased in elderly animals (older than 16 weeks) (Huang et al., 2014; Wang et al., 2006). Lack of signal in different brain areas (e.g. hypothalamus and motor nuclei) of adult TRPV1-PLAP-LacZ reporter mice compared to abundant signal in TRPV1-Cre/Rosa26-LacZ reporter mice could reflect a transient expression of TRPV1 during earlier development stages, which could greatly exceed that during adulthood (Cavanaugh et al., 2011). It has been argued that TRPV1 expression in adult mice is far lower in the brain compared to sensory neurons of the DRG in general, where the hippocampus has 


\section{INTRODUCTION}

the lowest levels of TRPV1 protein in the CNS (Han et al., 2012; Menigoz and Boudes, 2011; Roberts et al., 2004; Sanchez et al., 2001).

\subsubsection{TRPV1 splice isoforms}

Alternative splicing is a fundamental mechanism of eukaryotic cells that plays a central role in transcriptome variation allowing proteomic diversity by removing or inserting exons of the transcribed mRNA resulting in the translation of isoforms or splice variants of the full-length protein. Since isoforms have different amino acid sequence and even conformational states, they generally have different function than the full-length protein (Black, 2003; Gamazon and Stranger, 2014). At least 5 different isoforms of TRPV1 have been found in mammals: VR.5' sv (Schumacher et al., 2000), TRPV1 $\beta$ (Wang et al., 2004), TRPV1var (Tian et al., 2006), $\Delta-\mathrm{N}$ TRPV1 (Naeini et al., 2006) and TRPV1-S (Gracheva et al., 2011). VR.5' sv is the most likely splice isoform to be present in the CNS (Sanchez et al., 2001; Schumacher et al., 2000). Messenger RNA levels of TRPV1 $\beta$ were present in the same amounts as TRPV1 in DRG and brain but the protein for this splice isoform was much lower than TRPV1, denoting a possible instability of the protein; TRPV1 $\beta$ negatively regulates TRPV1 gating upon capsaicin and low $\mathrm{pH}$ (Wang et al., 2004). The unique osmosensing nature of the $\Delta-\mathrm{N}-\mathrm{TRPV} 1$ splice isoform confirms that transcriptome diversity of a single protein can profoundly change the functionality of the spliced variant. The C-terminal TRPV1 splice isoform TRPV1-S, which is exclusively found in the trigeminal ganglia of the vampire bat Desmodus rotundus is a rare case of alternative splicing of TRPV1 in the animal kingdom. TRPV1-S lacks 62 amino acids of the carboxyl terminus, is activated at $30^{\circ} \mathrm{C}$ and strikingly, is proposed to confer the unique ability of vampire bats to sense infrared light (Gracheva et al., 2011). 


\section{INTRODUCTION}

\subsubsection{VR.5' sV}

The first discovered TRPV1 isoform was the 2,768 bp vanilloid receptor 5' splice variant (VR.5' sv) (Schumacher and Eilers, 2010; Schumacher et al., 2000). The identified cDNA lacks the first four exons of the full-length protein and the 60-amino acid long seventh exon (Schumacher et al., 2000; Xue et al., 2001). Relative to the TRPV1 cDNA sequence the VR5'sv sequence starts at the 588th bp. TRPV1 and VR5' sv cDNA are identical from the bp 681 to the end of the TRPV1 cDNA (nucleotide 2,847), except for one VR.5' sv-specific insertion and one deletion (Schumacher et al., 2000). The VR.5' sv sequence has an extra 575 bp stretch (including a polyadenylation signal sequence) but this represents a non-coding region (Schumacher et al., 2000). Apart from the extra non-coding VR.5' sv stretch, the VR.5' sv cDNA sequence has 2 major differences compared to TRPV1. First, after the VR.5' sv nucleotide 246 there is a 100 bp-long divergent VR.5' sv region and second, there is a deletion of TRPV1 nucleotides 1121 to 1301 that corresponds to the open reading frame (Schumacher et al., 2000).

The transcriptional start site of the full-length TRPV1 transcript is within exon 2 (not present in VR.5' sv), while the transcriptional start site of VR.5' sv is within exon 6, specifically at Met 308 , thus the resulting VR.5' sv protein would have a shorter Nterminus lacking almost all of the ankyrin repeats (except for a portion of the third one) that are crucial for the interaction with different ligands (Xue et al., 2001). Apart from the truncation of the N-terminus, VR.5' sv is identical to TRPV1

containing the same transmembrane domains, pore region and C-terminus (Schumacher et al., 2000; Xue et al., 2001) (see Figure 1). 
TRPV1

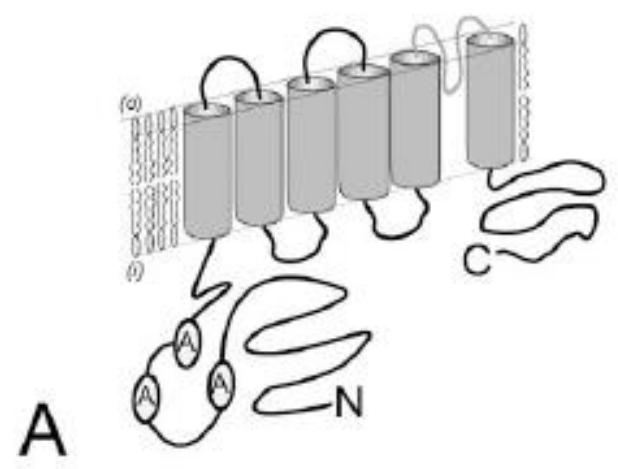

VR.5'sv

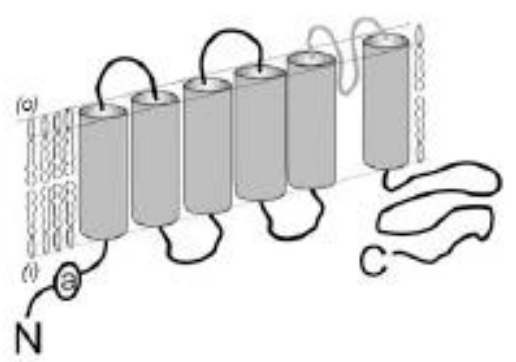

Figure 1. Diagram of the putative structure of TRPV1 and VR.5' sv. When compared VR.5' sv has the complete C-Terminus of TRPV1 but lacks most of the N-Termins. VR.5' sv has an insert in the N-T that is absent in the TRPV1 full-length protein. Figure modified from (Schumacher and Eilers, 2010).

Even though there is compelling evidence that the $\mathrm{N}$-terminus is necessary for the insertion of TRPV1 in the plasma membrane upon NGF treatment and $\mathrm{Ca}^{2+}$ influx (Hellwig et al., 2005; Zhang et al., 2005), transfected VR.5' sv has been observed to co-localize with TRPV1 inserted in the plasma membrane were it is proposed to heteromerize with TRPV1 (Eilers et al., 2007). Unlike TRPV1, monomeric VR.5' sv is not activated by noxious heat or vanilloids such as capsaicin or resiniferatoxin, however when TRPV1 and VR.5' sv are co-transfected in either Xenopus oocytes or HEK cells there is a $70 \%$ reduction in the response to these stimuli, which prompted the notion that VR.5' sv acts as a dominant negative TRPV1, stabilizing the channel in a more closed state (Eilers et al., 2007; Schumacher et al., 2000).

Interestingly, mRNA transcripts of VR.5' sv were found in the PNS as well as in the CNS (Sanchez et al., 2001; Schumacher et al., 2000). In DRGs TRPV1 mRNA levels have been found to exceed those of VR.5' sv by twelve to twenty times, however the ratio of mRNA for both transcripts is 1:1 in the brain (Sanchez et al., 2001; Schumacher et al., 2000) as well as in individual brain regions such as the hypothalamus, hippocampus, cerebellum, cerebral cortex, pons and medulla oblongata (Schumacher et al., 2000). As stated above the expression of TRPV1 has been reported to be greater in the PNS compared to the CNS. Interestingly, 


\section{INTRODUCTION}

VR.5' sv mRNA levels are the same in PNS (DRGs) and the brain (Sanchez et al., 2001; Schumacher et al., 2000).

\subsubsection{TRPV1 and synaptic plasticity}

\subsubsection{Synaptic plasticity}

With his observations almost one and a half centuries ago, Ramón y Cajal proposed a radical way to store information in neural tissue: changes in the connections between neurons (Cajal, 1894), later defined as synapses. Two different kinds of synapses have been described: electrical and chemical synapses. Typically chemical synapses consist of axon-dendrite connections; neurotransmitter is released from synaptic vesicles at the axonal pre-synapse and binds to receptors in the post-synapse on the dendrite. While synaptic transmission is unidirectional (pre- to post- synaptic), retrograde messengers (post- to pre synaptic) were later identified that modulate, for instance, presynaptic neurotransmitter release (Bailey et al., 2015).

The term synaptic plasticity was first proposed as a theoretical model to explain learning and memory (Hebb, 1949), whereby potentiation of the connection between two neurons results from the coincident activity of both neurons. The two best-characterized forms of synaptic plasticity are long-term potentiation (LTP) and long-term depression (LTD) of synaptic strength. Both LTP and LTD are important for memory formation. LTP is likely to underlie the formation and/or reactivation of memories, whereas LTD has been proposed to deactivate the same "remembered" memory attained by LTP (Nabavi et al., 2014). LTP can be induced by high frequency stimulation (50-100 Hz) leading to enhanced synaptic transmission. On the other hand, LTD is induced by low frequency stimulation $(1-5 \mathrm{~Hz})$ and leads to a decrease in synaptic transmission. Both types of synaptic plasticity are mediated by ion channels, namely the $\mathrm{Ca}^{2+}$-permeable NMDA receptor, which is essential for 


\section{INTRODUCTION}

either LTP or LTD in the hippocampus. Recently TRPV1 has been reported to mediate LTP and LTD in the hippocampus.

\subsubsection{TRPV1: LTP and LTD}

TRPV1 was first reported to influence hippocampal synaptic plasticity following the observation that induction of LTP after high frequency stimulation $(100 \mathrm{~Hz} / \mathrm{s})$ was reduced in TRPV1 KOs compared to WT (Marsch et al., 2007). In addition, capsaicin and resiniferatoxin facilitate the induction (but not the maintenance) of LTP induced by high frequency ( $\mathrm{Li}$ et al., 2008) and theta burst (Bennion et al., 2011) stimulation, and suppress LTD (induced by $10 \mathrm{~Hz} / \mathrm{s}$ low frequency stimulation), and these effects were blocked by the TRPV1 antagonists capsazepine and SB366791. Besides facilitating LTP, intrahippocampal capsaicin injections also improved spatial memory retrieval in rats subjected to stressful conditions (Li et al., 2008)..

These findings support the idea that TRPV1 is expressed in the hippocampus. But they did not explain where in the hippocampus TRPV1 is expressed and how it affects LTP. One possible mechanism has been identified: it appears likely that TRPV1-modulated LTP acts on hippocampal inhibitory interneurons that may affect LTP induction. HFS (two trains of $1 \mathrm{sec}$ at $100 \mathrm{~Hz}$ with $20 \mathrm{sec}$ interval) and $1 \mu \mathrm{M}$ capsaicin caused LTD of interneurons in the stratum radiatum, which was blocked by TRPV1 antagonists and did not occur in TRPV1 KOs (Gibson et al., 2008; McMahon and Kauer, 1997). According to the findings of Gibson et al., 2008 a model was proposed: LTD in inhibitory interneurons is independent of NMDA and AMPA receptors but is rather dependent on activation of mGluR1 receptors (and subsequent $\mathrm{Ca}^{2+}$ influx) known to be postsynaptically present in these neurons (Ferraguti et al., 2004). Postsynaptic mGluR1 activation upon HFS leads to synthesis of 12-HPETE by the 12-lipoxygenase enzyme using arachidonic acid as a metabolic substrate (Gibson et al., 2008); 12-HPETE was then proposed to retrogradely activate presynaptic TRPV1 (in pyramidal neurons), thus decreasing 


\section{INTRODUCTION}

the release of glutamate from CA1 and/or CA3 pyramidal neurons onto the interneuron (detected by alterations in the paired pulse ratio) (Gibson et al., 2008). LTD of interneurons of the stratum radiatum upon HFS and/or direct TRPV1 activation would then decrease the effects of feedforward inhibition from these neurons to CA1 pyramidal neurons, thus facilitating LTP, and explaining the reduction of LTP in TRPV1 KOs (Kauer and Gibson, 2009).

In fact, the enhanced LTP following TRPV1 activation with $1 \mu \mathrm{M}$ capsaicin or resiniferatoxin in the CA1 region of the hippocampus were reverted by $\mathrm{GABA}_{A}$ receptor blockade with picrotoxin (Bennion et al., 2011). Moreover the decrease in LTP observed in TRPV1 KO hippocampal slices was rescued with picrotoxin (Brown et al., 2013). Together these studies suggest that TRPV1 modulates LTP by reducing fast GABAergic feedforward inhibition from inhibitory interneurons onto CA1 pyramidal neurons.

In the dentate gyrus, an important center in the hippocampus that receives information from the entorhinal cortex, TRPV1 was found to underlie a form of post-synaptic LTD - induced by a $1 \mathrm{~Hz}$ paired pulse protocol or $1 \mu \mathrm{M}$ capsaicin - in granule cells innervated by the medial perforant path. This LTD was reverted with pretreatment with capsaicin to desensitize TRPV1, or with $10 \mu \mathrm{M}$ capsazepine (Chávez et al., 2010). Furthermore, this form of LTD was absent in acute hippocampal slices of TRPV1 KO mice (Chávez et al., 2010) and in slices from animals subjected to intrahippocampal injection of TRPV1 knockdown shRNA (Chávez et al., 2014). In contrast to the TRPV1-dependent LTP mechanism proposed in the CA1 region, in the dentate gyrus, activation of post-synaptic mGluR5 receptors by pre-synaptic glutamate release led to synthesis of postsynaptic anandamide that intracellularly activates TRPV1 inserted either in the plasma membrane or in the endoplasmic reticulum, subsequently causing an elevation of $\mathrm{Ca}^{2+}$ that ultimately induces calcineurin and dynamin-dependent endocytosis of AMPA receptors (Chávez et al., 2010; Marzo, 2010). This form of TRPV1-dependent post-synaptic LTD is in principle very similar to that described in 


\section{INTRODUCTION}

the nucleus accumbens (Grueter et al., 2010), although the induction protocols vary; LTD in the nucleus accumbens was induced by low frequency stimulation, while LTD in the dentate gyrus was induced by pre-synaptic burst stimulation of neurons from the perforant path together with post-synaptic depolarization (Chávez et al., 2010).

TRPV1 activation and inhibition respectively attenuate and increase LTD of inhibitory interneurons in the CA1 region of the hippocampus (Bennion et al., 2011; $\mathrm{Li}$ et al., 2008). Two different effects of capsaicin were observed during LTD induction in the CA1 region. Earlier studies showed that $1 \mathrm{mM}$ capsaicin causes a decrease in LTD (Li et al., 2008). However, $1 \mathrm{mM}$ capsaicin greatly exceeds the more physiological concentration that is sufficient to elicit gating of the channel (Gibson et al., 2008), In later studies, using more physiological concentrations of capsaicin $(1-10 \mu \mathrm{M})$ no significant alteration in the induction of LTD was detected (Bennion et al., 2011).

A recent study provides evidence that TRPV1 also influences inhibitory synaptic plasticity in the dentate gyrus. In this study, TRPV1 activation with $2 \mu \mathrm{M}$ capsaicin and $30 \mu \mathrm{M}$ anandamide (co-applied with the FAAH synthesis inhibitor URB597) depressed somatic but not dendritic inhibitory postsynaptic currents (IPSCs) in both the rat and mouse dentate gyrus. Pre-application of $10 \mu \mathrm{M}$ capsazepine - a competitive antagonist of capsaicin -, TRPV1 knockdown, and TRPV1 knockout, blocked this depression (Chávez et al., 2014). This TRPV1-mediated depression of inhibitory synaptic plasticity was proposed to occurred post-synaptically, via $\mathrm{Ca}^{2+}$ /calcineurin-dependent internalization of GABA receptors (Chávez et al., 2014), similar to the mechanism described for TRPV1-mediated post-synaptic LTD in the dentate gyrus, which was proposed to occur through AMPA receptor endocytosis (Chávez et al., 2010).

Even though recent discouraging studies have denied the presence of TRPV1 in the hippocampus (Cavanaugh et al., 2011), thus discarding TRPV1 as a major 


\section{INTRODUCTION}

player in modulating and/or mediating synaptic plasticity in the hippocampus, compelling new studies continue to confirm TRPV1 as a novel participant in synaptic plasticity in the hippocampus, opening the field to the possibility that not just TRPV1 but other TRP channels might account for finely tuning different forms of synaptic plasticity in multiple regions, subregions and neuronal populations.

\subsection{TRPV1 AND NEUROTROPHINS}

Neurotrophins can be defined as proteins that promote neuronal cell survival and differentiation. Yet, mounting evidence shows that neurotrophins are not limited to these actions but they are also involved in modulating synaptic plasticity and other physiologically relevant mechanisms such as pain processing and transduction, acting directly or indirectly on TRPV1 channels (Lewin and Nykjaer, 2014; Park and Poo, 2013).

Four types of neurotrophins have been characterized; the first one to be discovered was NGF (Levi-Montalcini and Hamburger, 1951), followed by BDNF (Barde et al., 1982) and finally neurotrophin 3 (NT3) and 4 (NT4) (Lewin and Barde, 1996; Park and Poo, 2013). All neurotrophins bind to the transmembrane receptor p75 and individually each neurotrophin binds to its specific transmembrane tropomyosin receptor; NGF binds to TrkA, BDNF and NT4 bind to TrkB and NT3 binds to TrkC (Chao, 2003; Lewin and Nykjaer, 2014; Park and Poo, 2013).

For each neurotrophin both the immature (pro) and mature forms exist. Although it was traditionally thought that pro-neurotrophins were only an uncleaved precursor of mature neurotrophins, increasing evidence suggests that pro-neurotrophins, actively participate in different biological functions in addition to their mature counterparts (Lee et al., 2001; Teng et al., 2010). Most of the literature suggests that immature forms of neurotrophins exclusively interact with the p75-sortilin 


\section{INTRODUCTION}

receptor complex and not the tropomyosin kinase receptors (Boutilier et al., 2008). Still it is unknown if certain conditions allow the activation of Trk receptors by proneurotrophins (Nykjaer et al., 2004)

Neurotrophins and their receptors are expressed in a cell-specific manner cell; depending on their localization they can exert different physiological functions. Neurotrophins are necessary for the normal development and function of neuronal networks. They promote neuronal differentiation and proliferation, including the specification of neuronal diversity; they are also involved in axonal and dendritic growth as well as in synaptogenesis and activity-dependent synaptic plasticity (Lewin, 1996; Lu et al., 2005; Park and Poo, 2013). Of all neurotrophins, NGF and BDNF have been most studied. Here I will focus on these two neurotrophins and the interactions of their mature forms with TRPV1 and TRPV1-related mechanisms.

\subsubsection{NGF as a regulator of TRPV1}

\subsubsection{NGF and TRPV1 expression: sensory neurons and hippocampus}

NGF is an integral component of nociception and neuropathic pain. It also is a crucial factor during inflammation and post-inflammatory symptoms like hyperalgesia (increased sensitivity to pain) (Lewin and Mendell, 1994; Lewin and Nykjaer, 2014). During early development NGF is an essential trophic factor for neuronal survival and differentiation (Cohen et al., 1954; Levi-Montalcini and Hamburger, 1951). During adulthood neurons stop depending on NGF for survival but neurons continue to express the TrkA receptor (for example in small-sized primary afferent $\delta$ and $C$ neurons in the DRG, which are also positive for TRPV1). Upon tissue and nerve injury (and subsequent inflammation) NGF is increased de novo in keranocytes and mast cells from where it is rapidly released into the affected body area. As NGF levels rise it binds to TrKA receptors present in these 


\section{INTRODUCTION}

nociceptors thus serving as a molecular messenger that ultimately elicits pain and hyperalgesia (Woolf and Costigan, 1999).

The undeniable requirement of NGF in pain perception and thermal hyperalgesia has long been recognized, but how NGF signaling is transduced into increased pain is still not completely understood. During thermal hyperalgesia, sensitization of nociceptors (i.e TRPV1) by NGF has been proposed to happen in two ways. As NGF increases it binds to the high-affinity TrKA receptor, which activates PKC and $\mathrm{PIP}_{3}$, which are considered to be the most likely candidates for sensitization of sensory neurons to pain (Lewin and Nykjaer, 2014). PKC is thought to directly sensitize TRPV1 through phosphorylation, thus leading (for example) to an increased neuronal response upon capsaicin stimulation (Numazaki et al., 2003). On the other hand $\mathrm{PIP}_{3}$ activation also activates Src kinase that in turn phosphorylates intracellularly located TRPV1 channels leading to their translocation to the plasma membrane (Zhang et al., 2005).

Although $\mathrm{PIP}_{3}$ increases the insertion of TRPV1 channels into the plasma membrane without affecting TRPV1 expression, other studies have reported increased expression of TRPV1 at both mRNA and protein levels (Anand et al., 2006; Winston et al., 2001). Upregulation of TRPV1 expression has been detected during nerve injury (Hudson et al., 2001) hyperalgesia (Davis et al., 2000; Miranda et al., 2007) and inflammatory pain (Ji et al., 2002; Luo et al., 2004; Schicho et al., 2004; Schwartz et al., 2013). In the case of inflammatory pain, NGF is as an important factor that mediates the upregulation of TRPV1 expression (Anand et al., 2006; Ji et al., 2002; Winston et al., 2001).

Information regarding the regulation of TRPV1 expression in the CNS, especially in the hippocampus is scarce and information about a possible mechanism causing an increase in TRPV1 expression is to my knowledge absent. Only a few studies have pointed out that TRPV1 expression is increased in patients with mesial temporal lobe epilepsy (Sun et al., 2012) and after seizures in rat models for 


\section{INTRODUCTION}

temporal lobe epilepsy (Bhaskaran and Smith, 2010). A more recent study found that mRNA levels of TRPV1 (together with receptors of the endocannabinoid system) were increased in brain and hippocampus of adult rats subjected to stress in early life (Marco et al., 2014).

NGF is expressed in brain and hippocampus (Gall and Isackson, 1989; Korsching et al., 1985). In the hippocampus NGF expression has been observed in inhibitory neurons expressing TRPV1 in the human hippocampus (Sun et al., 2012), and in a subset of inhibitory interneurons in multiple regions including neurons in the stratum oriens (SO) (Bizon et al., 1999; Pascual et al., 1998) that receive axonal projections from cholinergic septohippocampal neurons (Rocamora et al., 1996); these NGF expressing neurons in the SO correspond to high-expressing TRPV1OLM neurons. Thus it is likely that NGF could drive TRPV1 expression in OLM neurons, since it is known that neurons expressing TRPV1 in DRGs, in addition to a wide variety of nociceptive modulators, co-express TrKA (Ernsberger, 2009), which is necessary for proper maintenance of TRPV1 expression levels in nociceptors (upon its binding to NGF) (Luo et al., 2007).

\subsubsection{BDNF}

Evidence of BDNF affecting TRPV1 activity and/ or expression levels or vice versa is scarce, compared to the information regarding NGF driven TRPV1 expression and sensitization. However one can hypothesize that an interaction between BDNF and TRPV1 is likely, considering that in DRGs BDNF is expressed in a population of small- to medium-sized sensory neurons (Barakat-Walter, 1996; Merighi et al., 2008; Obata and Noguchi, 2006; Wetmore and Olson, 1995) including a population of TRPV1-positive cells (Xia et al., 2012) and TrkA-expressing neurons (Apfel et al., 1996; Michael et al., 1997). BDNF has been associated with neuroinflammatory pain (Xia et al., 2012; Zhao et al., 2006), hyperalgesia (Groth and Aanonsen, 2002), neuropathic pain (Fukuoka et al., 2001; Khan and Smith, 2015; Obata and Noguchi, 2006), and inflammation (Cho et al., 1997a, 1997b). In 


\section{INTRODUCTION}

neurons from the DRG, NGF - in addition to driving TRPV1 expression - can also regulate expression of BDNF (Apfel et al., 1996; Merighi et al., 2008; Michael et al., 1997). Similar to TRPV1, BDNF is also upregulated during inflammation (Cho et al., 1997a, 1997b), axotomy (Michael et al., 1997; Tonra et al., 1998), hyperalgesia (Yajima et al., 2002) and neuroinflammatory pain (Xia et al., 2012). In neuronal pain models, BDNF is known for its pronociceptive properties (reviewed in Khan and Smith, 2015) possibly by decreasing desensitization of TRPV1 channels (Ciobanu et al., 2009; Obata and Noguchi, 2006); activation of TRPV1 on the other hand has been reported to cause an increase in BDNF release (Lever et al., 2001). Although one study suggested that BDNF increases TRPV1 expression in DRG cells (Ciobanu et al., 2009), whether BDNF could potentially control TRPV1 expression remains uncertain (Price et al., 2005).

In the mammalian brain BDNF as well as NGF expression is regulated by neuronal activity (Park and Poo, 2013; Zafra et al., 1990). For instance BDNF expression upon depolarization is in part achieved through the activation of $\mathrm{Ca}^{+}{ }^{+}$-dependent transcription factors such as the cAMP response element-binding protein (CREB) that binds to corresponding regulatory elements upstream of the bdnf gene sequence (Tao et al., 1998, 2002).

BDNF promotes neuronal differentiation (rather than survival) of neural stem cells in brain and hippocampus, including the differentiation of inhibitory interneurons (Ahmed et al., 1995; Jones et al., 1994). BDNF also promotes the formation of neurites into axons during axon-dendrite differentiation (Shelly et al., 2007). In addition, BDNF stimulates synaptogenesis of both excitatory and inhibitory synapses in hippocampal neurons (Vicario-Abejón et al., 1998). Interestingly BDNF also aids to the maturation of excitatory and inhibitory synapses, since both a depolarization-induced release of endogenous BDNF, as well as exogenous BDNF application turn non-functional contacts between hippocampal neurons into functional synapses (Sallert et al., 2009; Vicario-Abejón et al., 1998). 


\section{INTRODUCTION}

In mature developmental stages BDNF differentially regulates excitatory and inhibitory synaptic efficiency by facilitating glutamatergic and depressing GABAergic synaptic transmission by modifying either pre- or postsynaptic strength (Bolton et al., 2000; Boulanger and Poo, 1999; Levine et al., 1995). BDNF is essential for LTP in the hippocampus; BDNF KO animals have an impairment in LTP induction that can be rescued with exogenous application of recombinant BDNF (Korte et al., 1995; Kovalchuk et al., 2002; Patterson et al., 1996).

There is increasing evidence that neuronal activity has a pivotal role in synaptic transmission that can ultimately lead to changes in the number and strength of synapses (Bliss and Collingridge, 1993; Linden, 1994; Malenka and Nicoll, 1999). Neuronal activity also promotes BDNF expression and secretion (Patterson et al., 1992; Zafra et al., 1990) especially at synapses where it contributes to an enhancement in synaptic transmission (Bonhoeffer, 1996; Katz and Shatz, 1996; Thoenen, 1995); in excitatory synapses BDNF release can also occur from the post- to the pre-synaptic part, thus serving as a retrograde messenger modulating pre-synaptic neurotransmitter release.(Hartmann, 2001; Matsuda et al., 2009).

Although the literature of BDNF and its actions during the elevation of neuronal activity in synaptic plasticity and plasticity is extensive, there is currently no evidence for a link between BDNF and TRPV1 expression in the hippocampus.

\subsection{ORIENS LACUNOSUM MOLECULARE (OLM) INTERNEURONS}

OLM interneurons are a subclass of interneurons located in the CA1 and CA3 region of the stratum oriens, close to the border with the alveolus; they were described already by Ramón y Cajal as neurons with big somatas with axons extending to the stratum lacunosum moleculare (Cajal, 1894; Müller and Remy, 
2014). They have horizontal dendrites projecting through the stratium oriens but also to further areas of the CA1 region (Freund and Buzsáki, 1996).

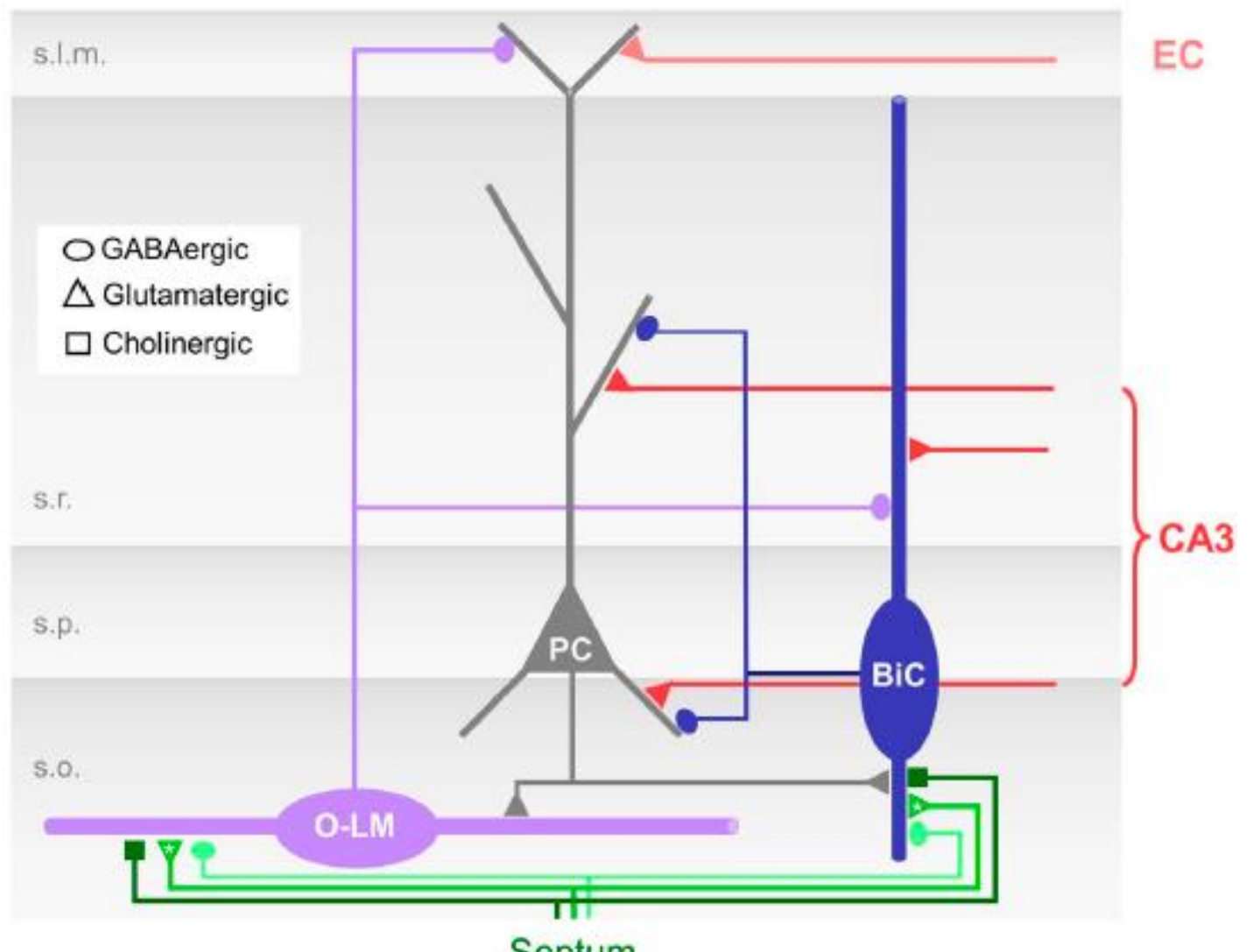

Septum

Figure 2. Diagram of a CA1 microcircuit involving OLM neurons. OLM neurons (purple) located in the stratum oriens (s.o.) receive glutamatergic input from pyramidal neurons (PC; grey) and cholinergic input coming from septal regions (dark green). OLM neurons send inhibitory projections to putative bistratified (BiC; blue) interneurons in the stratum radiatum (s.r.) and to distal dendrites of pyramidal neurons in the stratum lacunosum moleculare (s.I.m). Figure modified from (Müller and Remy, 2014)

OLM neurons seem to be a typical feedback inhibitory interneuron, predominantly receiving inputs from pyramidal neurons of the CA1 region (with few synapses from the CA3) (Sun et al., 2014) (Figure 2). Furthermore as they also receive cholinergic input from the medial septum and diagonal band of Broca. OLM neurons express both nAChr2 and nAChr7, thus making them sensitive to nicotine and acetylcholine (Leão et al., 2012) (Figure 2). The inhibitory input of OLM neurons has not been fully described, however it has been noted that they receive inhibition from other OLM neurons and bistratified neurons (Acsády et al., 1996; Ferraguti et al., 2004; 


\section{INTRODUCTION}

Kogo et al., 2004; Leão et al., 2012). OLM interneurons mostly inhibit distal dendrites of CA1 pyramidal neurons in the stratum lacunosum moleculare but in less proportion they also contact basal dendrites located in the stratum oriens (Freund and Buzsáki, 1996; Sik et al., 1995). Although most of the projections of OLM neurons are sent to pyramidal neurons, they also contact inhibitory interneurons, including feedforward inhibitory (presumably bistratified) interneurons in the stratum radiatum (Bezaire and Soltesz, 2013; Katona et al., 1999; Leão et al., 2012) (Figure 2).

OLM neurons are thought to be a key player in the generation of theta oscillations (4-12 Hz), and appear to create cross frequency coupling (from slow theta to fast spiking gamma frequency) and to gate LTP (Gloveli et al., 2005; Müller and Remy, 2014; Rotstein et al., 2005; Tort et al., 2007; Wulff et al., 2009). Specifically, OLM neurons control the information flow into pyramidal neurons of the CA1 from the entorhinal cortex and from the Schaffer collateral by directly inhibiting distal dendrites of CA1 neurons in the temporoammonic pathway from the entorhinal cortex, and disinhibiting the Schaffer collateral pathway via connections to other interneurons (Leão et al., 2012). Recently OLM neurons have been found to be recruited and activated during sharp wave ripple events of large amplitude and low frequency that simultaneously occur with reactivation of place cells correlated with high frequency ripple oscillations (Buzsáki et al., 1992; O'Neill et al., 2008; Pangalos et al., 2013). 


\section{MATERIALS AND METHODS}

\subsection{EXPERIMENTAL ANIMALS}

Lab rats used for experimentation were obtained from the UMG animal facility. All mice lines used for this research were bred and housed at the animal facility of the European Neuroscience Institute (ENI) Göttingen. Use of animals for experimentation was approved and performed following specifications of the Institutional Animal Care and Ethics Committees of Göttingen University (T10.31), and of the German animal welfare laws.

\subsubsection{Mouse lines}

TRPV1 ${ }^{-1}$ (TRPV1 KO) (Caterina et al., 2000) from B6/J strain. Purchased from Jackson Laboratory®.

TRPV1 ${ }^{\text {tm1 } 1 \text { (cre)Bbm }}$ (TRPV1-Cre) (Cavanaugh et al., 2011) from B6/J strain. Purchased from Jackson Laboratory®.

GT(Rosa)26Sortm14(CAG-tdTomato)Hze (Rosa26-tomato) (Madisen et al., 2010a) from B6/J strain. Provided by Jochen Staiger at the Department of Neuroanatomy of the UMG.

TRPV1 $^{\text {tm1(cre)Bbm }} \times$ GT(Rosa)26Sor ${ }^{\text {tm14(CAG-tdTomato)Hze }}$. These strains were interbred at the animal facility of the ENI Göttingen. We refer to the resulting mouse strain as the TRPV1-tdTomato mouse strain. 


\subsubsection{Genotyping}

Tips of tails were clipped from P21 or anesthetized mice (prior to perfusion fixation; see section 3.5.5.1). Tails were lysed with $500 \mu$ lysis buffer $(100 \mathrm{mM}$ Tris- $\mathrm{HCl}(\mathrm{pH}$ 8.5), $5 \mathrm{mM}$ EDTA ( $\mathrm{pH} 8.0$ ), $0.2 \%$ [w/v] SDS and $200 \mathrm{mM} \mathrm{NaCl}$ ) mixed with $7 \mu \mathrm{l}$ proteinase $\mathrm{K}(10 \mathrm{mg} / \mathrm{ml})$ overnight at $55^{\circ} \mathrm{C}$ with agitation. Digested tails were then centrifuged at max speed with the Eppendorf centrifuge 5424 for 10 min at RT. Supernatant was precipitated with $500 \mu \mathrm{l}$ isopropanol, mixed and centrifuged at max speed for $10 \mathrm{~min}$. Precipitated DNA-pellet was washed with $70 \%[\mathrm{v} / \mathrm{v}]$ ethanol by centrifuging for $10 \mathrm{~min}$ at max speed. Ethanol was discarded and the DNA-

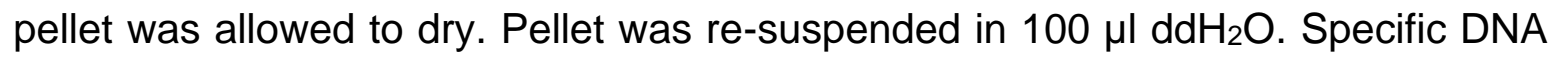
sequences were amplified using the polymerase chain reaction (PCR) (Mullis et al., 1986; Saiki et al., 1988) using an Eppendorf MasterCycler EP Gradient S PCR machine (Eppendorf).

Reaction mix used for qualitative PCR:

$2 \mu \mathrm{l} \quad$ genomic DNA

$5 \mu \mathrm{l} \quad$ 10X DreamTaq Buffer (Thermo Fisher Scientific)

$1 \mu \mathrm{l} \quad$ Forward primer $(10 \mu \mathrm{M})$

$1 \mu \mathrm{l} \quad$ Reverse primer $(10 \mu \mathrm{M})$

$0.4 \mu \mathrm{l} \quad$ dNTPs

$1.25 \mu \mathrm{l} \quad$ Taq polymerase (Thermo Fisher Scientific)

Reconstituted with $\mathrm{ddH}_{2} \mathrm{O}$ to a final volume of $50 \mu \mathrm{l}$

$25 \mu \mathrm{l}$ of each PCR reaction was loaded in independent wells of a solid agarose gel ( $1 \%[\mathrm{w} / \mathrm{v}]$ ultrapure agarose (Invitrogen) diluted in 1X TAE buffer) mixed with a 1:1000 ratio of Roti-safe fluorescent gel stain (Carl Roth) for visualization. For estimating the size of amplified DNA fragments a GeneRuler marker (Thermo Fisher) was loaded in separate wells. Subsequently, standard nucleic acid electrophoresis was conducted under constant voltage using a Bio-rad power supply. Amplified DNA fragments were visualized using a UV lamp on the Gel iX20 


\section{MATERIALS AND METHODS}

Imager (iNTAS). Amplicons obtained from DNA isolated from mutant mouse lines were always compared to the B6/J line (WT).

\subsubsection{PCR protocols and primers for genotyping}

Sequences of all primers for genotyping every mouse line used (see section 3.1.1), as well as the PCR cycling protocol for each primer pair were obtained from the JAX® Mice genotyping resource (The Jackson Laboratory). All primers (indicated below) were synthesized by and purchased from Sigma-Aldrich $\AA$. Primer pairs detecting WT and mutant genotypes were tested in both B6/J (WT) and mutant mouse DNA samples.

Primers used for genotyping the TRPV1 KO line:

WT Forward: 5'-cctgctcaacatgctcat-3'

Mutant Forward: $\quad$ 5'-tcctcatgcacttcaggaaa-3'

Common Reverse: $\quad$ 5'-tggatgtggattgtgtgcgag-3'

PCR protocol:

$94^{\circ} \mathrm{C} \quad 3 \mathrm{~min}$

$\left.\begin{array}{ll}94^{\circ} \mathrm{C} & 30 \mathrm{sec} \\ 65^{\circ} \mathrm{C} & 1 \mathrm{~min} \\ 72^{\circ} \mathrm{C} & 1 \mathrm{~min}\end{array}\right\} \times 35$

$72^{\circ} \mathrm{C} \quad 2 \mathrm{~min}$

$4^{\circ} \mathrm{C} \quad$ hold

Mutant $=450 \mathrm{bp}$

Heterozygous $=450$ and $984 \mathrm{bp}$

$\mathrm{WT}=984 \mathrm{bp}$ 


\section{MATERIALS AND METHODS}

Primers used for genotyping the TRPV1 Cre line:

WT Forward:

WT Reverse:

Mutant Forward:

Mutant Reverse 5'-ttcagggagaaactggaagaa-3'

5'-tagtcccagccatccaaaag-3'

5'-gcggtctggcagtaaaaactatc-3'

5'-gtgaaacagcattgctgtcactt-3'

PCR protocol:

$94^{\circ} \mathrm{C} \quad 2 \mathrm{~min}$

$94^{\circ} \mathrm{C} \quad 20 \mathrm{sec}$

$65^{\circ} \mathrm{C}$

$68^{\circ} \mathrm{C}$

$94^{\circ} \mathrm{C}$

$50^{\circ} \mathrm{C}$

$72^{\circ} \mathrm{C}$

$72^{\circ} \mathrm{C}$

$4^{\circ} \mathrm{C}$

$15 \mathrm{sec}$

X 10

$10 \mathrm{sec}$

$15 \mathrm{sec}$

$15 \mathrm{sec}$

$\times 28$

Mutant $=102 \mathrm{bp}$

Heterozygous $=102$ and $490 \mathrm{bp}$

$\mathrm{WT}=490 \mathrm{bp}$

Primers used for genotyping the Rosa26-tomato line:

WT Forward:

5'-aagggagctgcagtggagta-3'

WT Reverse:

5'-ccgaaaatctgtgggaagtc-3'

Mutant Forward:

5'-ctgttcctgtacggcatgg-3'

Mutant Reverse:

5'-ggcattaaagcagcgtatcc-3' 


\section{MATERIALS AND METHODS}

intracellular cleavage at the P2A site and subsequent co-expression (at equal ratios) of untagged TRPV1 and cytosolic EGFP, or of untagged VR.5' sv and cytosolic RFP. The $\beta$-actin promoter-driven TRPV1-P2A-EGFP and VR.5' sv-P2ARFP plasmids were sub-cloned by GenScript (Piscataway, NJ, USA).

For plasmid DNA isolation, electro-competent Escherichia coli bacteria were transformed with TRPV1, VR.5' sv, RFP or EGFP DNA constructs by electroporation. After transformation the bacteria were plated on polystyrene petri dishes (CytoOne) containing solidified LB-Agar consisting of $0.5 \%[\mathrm{w} / \mathrm{v}]$ yeast extract, $1 \%[\mathrm{w} / \mathrm{v}]$ Tryptone $(\mathrm{pH} 7.0), 1 \%[\mathrm{w} / \mathrm{v}] \mathrm{NaCl}$ and $1.5 \%[\mathrm{w} / \mathrm{v}]$ agar (Carl Roth). Transformed bacteria were allowed to grow overnight at $37^{\circ} \mathrm{C}$. Individual bacterial colonies were picked with a pipette tip and placed into $100 \mathrm{ml}$ LB medium including $50 \mu \mathrm{g} / \mathrm{ml}$ ampicillin (Carl Roth) for selection of bacteria with the plasmids above (which are ampicillin resistant) and cultured overnight at $37^{\circ} \mathrm{C}$ with shaking at $300 \mathrm{rpm}$ until an $\mathrm{OD}_{600}$ of 2 to 3 was achieved. $100 \mathrm{ml}$ of liquid LB medium containing bacterial cultures was used for plasmid DNA extraction with the NucleoBond Plasmid PC 500 maxi-prep kit (Macherey \& Nagel).

Following plasmid DNA isolation, double stranded DNA concentration was estimated using a spectrophotometer (NanoDrop ND-1000) with ND-1000 v3.5.2 software (peQLabBiotechnologie) to determine the amount of DNA to use in transfections.

\subsection{CELL CULTURE METHODS}

\subsubsection{Dissociated rat hippocampal neuron preparation}

E18 pregnant Wistar rats (UMG animal facility) were euthanized with $\mathrm{CO}_{2}$, the abdomen was opened and embryos were removed from the uterus. Embryos were collected from the amniotic sac; heads were cut off and placed in a $10 \mathrm{~cm}$ petri 


\section{MATERIALS AND METHODS}

dish containing cold dissection media (HBSS (Gibco) + 10 mM Hepes (Gibco)). Brains were removed from the skull and collected in fresh dissection media. Hippocampi were separated from the brain and meninges were removed. Hippocampi were digested with $2 \mathrm{ml}$ prewarmed $37^{\circ} \mathrm{C} 0.05 \%$ [w/v] trypsin-EDTA (Gibco) for 20 minutes at $37^{\circ} \mathrm{C}$. Trypsin was removed and the tissue was washed three times with $4^{\circ} \mathrm{C}$ dissection media. Dissection media was replaced with $1 \mathrm{ml}$ pre-warmed NB+ (Neurobasal (Gibco) with 1X B-27 supplement (Gibco), 1X Glutamax (Gibco) and Penicillin (5000 U/ml)/Streptomycin $(5000 \mu \mathrm{g} / \mathrm{ml}$, Gibco)) and tissue was triturated by gentle pipetting. The tissue suspension was filtered with $100 \mu \mathrm{m}$ cell strainer (BD Biosciences). $10 \mu \mathrm{l}$ of cell suspension was mixed with an equal volume of trypan blue, the number of living cells were counted using a hemacytometer (excluding blue cells) and the final concentration of neurons was calculated. Cells were cultured on $12 \mathrm{~mm}$ glass coverslips (Thermo Scientific) coated with poly-D-lysine (PDL, Sigma) dissolved in $0.1 \mathrm{M}$ borate buffer, in 24-well plates (CytoOne) at a density of 80,000 hippocampal neurons per $\mathrm{cm}^{2}$ and cultured in NB+ culture media in a Hera Cell 240i cell culture Incubator (Thermo Scientific) at $37^{\circ} \mathrm{C}$ and $5 \%[\mathrm{w} / \mathrm{v}] \mathrm{CO}_{2}$.

\subsubsection{Dissociated mouse hippocampal neuron preparation}

Neonate P0 B6/J mice litters were used for hippocampal neuronal preparation. Each pup was decapitated, and the head was placed on a petri dish and sprayed with $70 \%[\mathrm{v} / \mathrm{v}]$ ethanol. The skin of the scalp was separated, skull was opened, the brain was removed and placed in a petri dish containing $4^{\circ} \mathrm{C}$ dissection media. Every decapitation and brain dissection was done successively for each pup. Hippocampal dissection from the brain was performed similar to the protocol for rat embryonic hippocampal dissection with only few modifications. Instead of trypsinEDTA, filtered papain-based enzymatic solution (11.39 mM L-Cysteine, $50 \mathrm{mM} \mathrm{Na-}$ EDTA ( $\mathrm{pH}=8$ ), $100 \mathrm{mM} \mathrm{CaCl} 2,1 \mathrm{~N} \mathrm{NaOH}, 100 \mu \mathrm{l}$ papain (Worthington) (added to the solution $15 \mathrm{~min}$ before use) and $10 \mathrm{mg} / \mathrm{ml}$ of DNAsel added just before digestion) at $37^{\circ} \mathrm{C}$ was used for tissue digestion (for $30 \mathrm{~min}$ at $37^{\circ} \mathrm{C}$ ). Following the 


\section{MATERIALS AND METHODS}

incubation the enzymatic solution was removed and replaced by an inactivation solution (BSA, $5 \%$ serum medium and $10 \mathrm{mg} / \mathrm{ml}$ of DNAsel) to inactivate enzymatic components; the inactivation was achieved at $37^{\circ} \mathrm{C}$ for $15 \mathrm{~min}$. Subsequently the tissue was triturated as mentioned before, with a minor adjustment: the tissue suspension was centrifuged in an Eppendorf 5810-12 centrifuge (Eppendorf) at $500 \mathrm{~g}$, the supernatant was discarded and replaced with fresh $\mathrm{NB}_{+}$, and the tissue pellet was re-homogenized before plating. The counting and plating procedure was identical to that for rat embryonic hippocampal neuron preparation. Neurons were plated at a density of $120,000 \mathrm{cells} / \mathrm{cm}^{2}$.

\subsubsection{Human embryonic kidney 293 (HEK) cell culture preparation}

Frozen $\left(-80^{\circ} \mathrm{C}\right) \mathrm{HEK}$ cells were thawed quickly at $37^{\circ} \mathrm{C}$. After thawing, the cell suspension was re-homogenized in $10 \mathrm{ml} \mathrm{HEK} \mathrm{cell} \mathrm{medium} \mathrm{(DMEM} \mathrm{(Gibco),} \mathrm{10 \%}$ $[\mathrm{v} / \mathrm{v}]$ FBS, 2\% [v/v] Penicillin/Streptomycin). Suspended cells were centrifuged in an Eppendorf 5810-12 centrifuge (Eppendorf) at 4000 RPM for 3 min at RT. The supernatant was discarded and the cell pellet was re-suspended in $10 \mathrm{ml} 37^{\circ} \mathrm{C}$ HEK cell medium and plated in a $10-\mathrm{cm}$ tissue culture dish (Cellstar). Confluency was checked after 1-3 days in vitro; cells were normally split when cell confluency was approximately $50-60 \%$.

For splitting, culture medium was removed, cultures were washed once with sterile DPBS (Sigma-Aldrich) followed by trypsinization: $1 \mathrm{ml} 0.25 \%$ Trypsin-EDTA at $37^{\circ} \mathrm{C}$ was added to the cultures and incubated for $2-3 \mathrm{~min}$ at $37^{\circ} \mathrm{C}$. Cultures were then washed off of the plate with a pipette and collected in $10 \mathrm{ml} \mathrm{HEK}$ cell medium. Cell suspension was centrifuged in an Eppendorf 5810-12 centrifuge (Eppendorf) at $4000 \mathrm{RPM}$ for $3 \mathrm{~min}$ at RT. The supernatant was removed and the cell pellet was re-suspended in $10 \mathrm{ml} \mathrm{HEK}$ cell medium $\left(37^{\circ} \mathrm{C}\right) .250 \mu \mathrm{l}$ of the suspension was diluted in $9.75 \mathrm{ml} \mathrm{HEK}$ cell medium and plated in a new $10 \mathrm{~cm}$ tissue culture dish. 
MATERIALS AND METHODS

HEK cell cultures were allow to grow in a Hera Cell 240i cell culture incubator (Thermo Scientific) at $37^{\circ} \mathrm{C}$ and $5 \%[\mathrm{w} / \mathrm{v}] \mathrm{CO}_{2}$.

HEK cell cultures were transfected 1-2 days after plating following a protocol similar to the one used for transfecting hippocampal neurons (explained in section 3.4 ) with minor changes (i.e. HEK cell culture medium was used instead of NB or $\mathrm{NB}+)$.

\subsection{HETEROLOGOUS OVEREXPRESSION OF THE FULL LENGTH TRPV1 CHANNEL AND VR5 SPLICE ISOFORM.}

\subsubsection{Transfection of dissociated rat hippocampal neurons}

In order to achieve single neuron heterologous expression of different plasmids (mentioned in 3.2), lipofectamine based transfections were performed in rat or mouse hippocampal neurons cultured in vitro (as previously mentioned). Transfections were conducted either at DIV 2 or DIV 10 (depending on the experiment).

Single plasmid transfections were carried out with $0.75 \mu \mathrm{g} /$ well of plasmid DNA (for co-transfections, $0.35 \mu \mathrm{g} /$ well of DNA was used for each construct) and $1 \mu \mathrm{l} /$ well of the cationic lipid-based transfection reagent lipofectamine 2000 (Invitrogen). These volumes of lipofectamine 2000 and of plasmid DNA were each diluted in $50 \mu \mathrm{l} /$ well of $4^{\circ} \mathrm{C}$ Neurobasal in separate tubes and incubated for 5 min at RT. Subsequently, both dilutions were mixed together and incubated at RT for 20 min. During this incubation time, the culture medium from each well to be transfected was exchanged with $400 \mu \mathrm{l}$ of new prewarmed $\mathrm{NB}+$ at $37^{\circ} \mathrm{C}$; the original culture medium was not discarded but stored in the cell culture incubator at $37^{\circ} \mathrm{C}$ and $5 \%$ [w/v] $\mathrm{CO}_{2}$. After incubation, $100 \mu \mathrm{l}$ of the lipofectamine-DNA mix was then added to each well for a final volume of $500 \mu \mathrm{l} /$ well. Cultures were incubated for $2 \mathrm{hr}$ at $37^{\circ} \mathrm{C}$ 
and $5 \%[\mathrm{w} / \mathrm{v}] \mathrm{CO}_{2}$ with the transfection medium. Afterwards, the transfection medium was removed, and wells were washed once with $500 \mu \mathrm{l} \mathrm{NB}$ at $37^{\circ} \mathrm{C}$ and $450 \mu \mathrm{l}$ of the original culture medium at $37^{\circ} \mathrm{C}$ was added back to the wells. Identification of reporter gene expression was normally detected 2 days after transfection.

\subsection{IMMUNOSTAINING METHODS}

\subsubsection{Primary antibodies}

Table 1 details all primary antibodies used for immunostaining and western blots during this project.

Table 1. List of primary antibodies.

\begin{tabular}{|c|c|c|c|c|}
\hline Antibody & Host (clonality) & $\begin{array}{c}\text { Final } \\
\text { dilution }\end{array}$ & Application & Source \\
\hline BDNF & $\begin{array}{c}\text { Mouse } \\
\text { (monoclonal) }\end{array}$ & $1: 100$ & ICC/WB & $\begin{array}{c}\text { Sigma- } \\
\text { Aldrich }\end{array}$ \\
\hline C-Fos & $\begin{array}{c}\text { Rabbit } \\
\text { (polyclonal) }\end{array}$ & $1: 300$ & ICC & Santa Cruz \\
\hline EGFP & $\begin{array}{c}\text { Chick } \\
\text { (polyclonal) }\end{array}$ & $1: 4000:$ & ICC & Abcam \\
\hline GAD65 & $\begin{array}{c}\text { Rabbit } \\
\text { (polyclonal) }\end{array}$ & $1: 4000:$ & ICC & Abcam \\
\hline Mouse & $1: 1000$ & ICC & $\begin{array}{c}\text { Synaptic } \\
\text { Systems }\end{array}$ \\
\hline MAP2 & $\begin{array}{c}\text { Chick } \\
\text { (polyclonal) }\end{array}$ & $1: 4000$ & ICC/IHC & Millipore \\
\hline Mouse & $1: 4000$ & ICC & Millipore \\
\hline
\end{tabular}


MATERIALS AND METHODS

\begin{tabular}{|c|c|c|c|c|}
\hline & (monoclonal) & & & \\
\hline mGluR7 & $\begin{array}{c}\text { Rabbit } \\
\text { (polyclonal) }\end{array}$ & $1: 500$ & ICC & Upstate \\
\hline NGF & $\begin{array}{c}\text { Sheep } \\
\text { (monoclonal) }\end{array}$ & $1: 1000$ & ICC & Millipore \\
\hline PV25 & $\begin{array}{c}\text { Mouse } \\
\text { (monoclonal) }\end{array}$ & $1: 2000$ & $\mathrm{ICC} / \mathrm{IHC}$ & Swant \\
\hline Reelin & $\begin{array}{c}\text { Mouse } \\
\text { (monoclonal) }\end{array}$ & $1: 1000$ & $\mathrm{ICC} / \mathrm{IHC}$ & Millipore \\
\hline SMI-312 & $\begin{array}{c}\text { Mouse } \\
\text { (monoclonal) }\end{array}$ & $1: 4000$ & ICC & Covance \\
\hline Somatostatin-14 & $\begin{array}{c}\text { Rabbit } \\
\text { (polyclonal) }\end{array}$ & $1: 2000$ & ICC/IHC & Bachem \\
\hline Somatostatin-14 & Rat (monoclonal) & $1: 1000$ & $\mathrm{ICC} / \mathrm{IHC}$ & $\begin{array}{l}\text { Thermo } \\
\text { Fisher }\end{array}$ \\
\hline Syt1 & $\begin{array}{l}\text { Guinea pig } \\
\text { (polyclonal) }\end{array}$ & $1: 800$ & Syt1u & $\begin{array}{l}\text { Synaptic } \\
\text { Systems }\end{array}$ \\
\hline Syt1 & $\begin{array}{c}\text { Mouse } \\
\text { (monoclonal) }\end{array}$ & $1: 150$ & Syt1u & $\begin{array}{l}\text { Synaptic } \\
\text { Systems }\end{array}$ \\
\hline TRPV1 (C-T) & $\begin{array}{l}\text { Guinea pig } \\
\text { (polyclonal) }\end{array}$ & $1: 1000$ & $\mathrm{ICC} / \mathrm{IHC}$ & Millipore \\
\hline
\end{tabular}

\subsubsection{Secondary antibodies}

Table 2 indicates all secondary antibodies used in all immunostaining and western blots conducted during this project. 
MATERIALS AND METHODS

Table 2. List of secondary antibodies.

\begin{tabular}{|c|c|c|c|c|}
\hline Antibody & $\begin{array}{c}\text { Host } \\
\text { species }\end{array}$ & $\begin{array}{c}\text { Final } \\
\text { dilution }\end{array}$ & Application & Source \\
\hline a-guinea pig HRP & Goat & $1: 10000$ & WB & Biorad \\
\hline a-mouse HRP & Goat & $1: 10000$ & WB & Biorad \\
\hline a-rabbit HRP & Goat & $1: 10000$ & WB & Biorad \\
\hline a-chick Alexa 405 & Goat & $1: 1000$ & ICC/IHC & Abcam \\
\hline a-rabbit Alexa 405 & Goat & $1: 1000$ & ICC/IHC & Abcam \\
\hline a-chick Alexa 488 & Goat & $1: 1000$ & ICC/IHC & Invitrogen \\
\hline a-guinea pig Alexa 488 & Goat & $1: 1000$ & ICC/IHC & Invitrogen \\
\hline a-mouse Alexa 488 & Donkey & $1: 1000$ & ICC/IHC & Invitrogen \\
\hline a-rabbit Alexa 488 & Goat & $1: 1000$ & ICC/IHC & Invitrogen \\
\hline a-guinea pig Alexa 546 & Goat & $1: 1000$ & ICC/IHC & Invitrogen \\
\hline a-mouse Alexa 546 & Donkey & $1: 1000$ & ICC/IHC & Invitrogen \\
\hline a-rabbit Alexa 546 & Goat & $1: 1000$ & ICC/IHC & Invitrogen \\
\hline a-sheep Alexa 546 & Donkey & $1: 1000$ & ICC/IHC & Invitrogen \\
\hline a-guinea pig Alexa 647 & Goat & $1: 1000$ & ICC/IHC & Invitrogen \\
\hline a-mouse Alexa 647 & Donkey & $1: 1000$ & ICC/IHC & Invitrogen \\
\hline a-rabbit Alexa 647 & Goat & $1: 1000$ & ICC/IHC & Invitrogen \\
\hline a-rat Alexa 546 & Goat & $1: 1000$ & ICC/IHC & Invitrogen \\
\hline
\end{tabular}

\subsubsection{Synaptotagmin 1 luminal domain antibody uptake}

Synaptotagmin 1 luminal domain antibody uptake (syt1u) is a method used to visually identify synaptic vesicle recycling, allowing us to estimate presynaptic changes in synaptic strength. Syt 1 is an integral membrane protein of synaptic 


\section{MATERIALS AND METHODS}

vesicles (Chapman, 2002; Koh and Bellen, 2003; Stein et al., 2007; Sutton et al., 1995). Since the N-terminus of Syt 1 is in the lumen of synaptic vesicles, antibodies specifically targeting the $\mathrm{N}$-terminus (luminal domain) of Syt 1 would bind and be taken up by recycling synaptic vesicles only when they fuse with the plasma membrane.

For syt $1 \mathrm{u}$, dissociated hippocampal neurons were incubated for 10 min at $37^{\circ} \mathrm{C}$ in $250 \mu \mathrm{l}$ high potassium "depolarizing" buffer $(75 \mathrm{mM} \mathrm{NaCl}, 70 \mathrm{mM} \mathrm{KCl}, 20 \mathrm{mM}$ Hepes, $2 \mathrm{mM} \mathrm{CaCl}$, $5,5 \mathrm{mM}$ glucose $2 \mathrm{mM} \mathrm{MgCl}_{2}$, and $20 \mathrm{mM}$ Hepes; $\mathrm{pH}$ 7.3) containing syt 1 luminal domain specific antibodies (see Table 1). Immediately afterwards, depolarizing buffer was removed and samples were fixed as detailed in section 3.5.4.

\subsubsection{Immunocytochemistry}

Dissociated hippocampal cultures growing on glass coverslips were fixed with 250 $\mu \mathrm{l} 4 \%[\mathrm{w} / \mathrm{v}]$ Paraformaldehyde (PFA) diluted in $0.1 \mathrm{M}$ phosphate buffer (PB) (0.1 M $\mathrm{Na}_{2} \mathrm{HPO}_{4}, 0.1 \mathrm{M} \mathrm{NaH}_{2} \mathrm{PO}_{4}$ dissolved in $\mathrm{ddH}_{2} \mathrm{O} ; \mathrm{pH}$ 7.4) for 20-30 minutes at room temperature. Subsequently, fixed hippocampal cell cultures were washed three times with $500 \mu \mathrm{l} 1 \mathrm{X}$ PBS (if necessary hippocampal cultures were stored up to a month at $4^{\circ} \mathrm{C}$ in $1 \mathrm{X} P B S$ ). Dissociated cultures were then blocked for 20 minutes at room temperature by incubation in $250 \mu \mathrm{l}$ blocking buffer containing Triton X-100 for permeabilization (2\% [v/v] donkey serum, 0,1\% [v/v] Triton X-100 (Carl Roth), $0,05 \%[\mathrm{v} / \mathrm{v}] \mathrm{NaN}_{3}$ in $\left.2 \mathrm{X} \mathrm{PBS}\right)$. Subsequently, samples were incubated with primary antibodies (see Table 1) diluted in blocking buffer overnight at $4^{\circ} \mathrm{C}$.

The solution containing primary antibodies was then discarded and cells were washed three times for 5 minutes each with $500 \mu \mathrm{l}$ 1X PBS. Cells were then incubated for $2 \mathrm{hr}$ at room temperature in the dark in $250 \mu$ l blocking buffer containing secondary antibodies conjugated to a fluorophore (see Table 2). Secondary antibody solution was then removed and cells were washed three times 


\section{MATERIALS AND METHODS}

for 5 min each with $1 \mathrm{X}$ PBS. If a DAPI staining was necessary samples were incubated for 10 minutes in $250 \mu \mathrm{l}$ X PBS containing $10 \mu \mathrm{M}$ DAPI (Invitrogen) at room temperature in darkness. Cells were again washed three times for 5 min each with 1X PBS. The same antibody mix (primary and secondary) was used for different conditions or treatments within the same experiment. Secondary antibodies used were crossed absorbed and previously tested to avoid crossreactivity.

Finally, coverslips were mounted with a drop $(10 \mu \mathrm{l})$ of Fluoromount/Plus (Diagnostic Biosystems) or Fluoromount-G (Sigma) on a glass slides (Carl Roth) for microscopy. Coverslips were sealed using transparent nail polish, and then imaged by epifluorescence and confocal microscopy (section 3.6).

\subsubsection{Immunohistochemistry}

\subsubsection{Paraformaldehyde perfusion fixation}

2-3 month old WT (B6J), TRPVI KO and TRPV1-Cre-Tomato mice were anesthetized by intraperitoneal injection of a tranquilizer cocktail containing $10 \%$ ketamine, $2 \%$ xylazine and $0.9 \%$ saline solution; volume injected depended on the weight of the animal $(0.1 \mathrm{ml} / 10 \mathrm{mg}$ body weight). After anesthetization mice were placed on a perfusion chamber (abdomen side up) and fastened with needles on the chamber. The rib cage was removed and once the heart was successfully exposed, the right atrium was cut and a winged needle connected to a peristaltic pump (Masterflex) was inserted into the left ventricle. The blood was drained by perfusion of pre-filtered sucrose solution ( $10 \%[\mathrm{w} / \mathrm{v}]$ sucrose (Carl Roth) in $\mathrm{dd}_{2} \mathrm{O}$ ) for ca. $1 \mathrm{~min}$ followed by ca. $50 \mathrm{ml}$ of pre-filtered 4\% PFA at RT for ca. $20 \mathrm{~min}$ (for fixation). A successful perfusion was detected when the tail of the animal became stiff. The brain was subsequently dissected out of the skull, incubated in sterilefiltered $4 \%$ PFA at $4^{\circ} \mathrm{C}$ for $2 \mathrm{hr}$, and washed twice for 15 min each with $0.1 \mathrm{M} \mathrm{PB}$. Tissue was then used for $\mathrm{IHC}$ or stored in $\mathrm{PB}$ at $4^{\circ} \mathrm{C}$ for up to a month. All buffers 


\section{MATERIALS AND METHODS}

and solutions used during this step were freshly prepared for every perfusion. Mice tails of all perfusions were clipped and genotyped (see 3.1.2).

\subsubsection{Acute brain slices}

Before tissue sectioning, fixed mouse brains (see section 3.5.5.1) were presectioned using an acrylic brain blocker (KOPF) to remove the most frontal and caudal parts of the brain to keep the starting point for slicing similar for every brain. Coronal brain slices were generated using a Leica VT 1200 vibratome (Leica Biosystems): Pre-sectioned brains were stabilized with glue Roti coll 1 (Carl Roth) caudally on a specimen disc fixed to the vibratome's buffer tray. The tray was then filled with $4^{\circ} \mathrm{C} 0.1 \mathrm{M} \mathrm{PB}$. The system was calibrated and $40 \mu \mathrm{m}$ thick coronal brain slices were cut using a razor blade (Astra Platinum). Each slice was collected in individual wells of a 24-well cell culture plate containing $4^{\circ} \mathrm{C} 0.1 \mathrm{M} \mathrm{PB}$ and stored up to a month at $4^{\circ} \mathrm{C}$.

Brain slices were visually selected for immunohistochemistry using an Axiovert 40 CFL (Zeiss). Comparable slices between different brains from different samples were sorted and classified depending on their approximate stereotactic coordinates according to Paxinos and Franklin's the Mouse Brain in Stereotaxic Coordinates (2012).

\subsubsection{Immunohistochemistry of mouse brain slices}

Slices were washed first with $50 \mathrm{mM}$ Tris buffer (TB) (38.5 mM Tris-HCl; $11.5 \mathrm{mM}$ in $\left.\mathrm{ddH}_{2} \mathrm{O} ; \mathrm{pH} 7.6\right)$, then with Tris buffered saline (TBS) $(0.9 \%[\mathrm{w} / \mathrm{v}] \mathrm{NaCl}$ in TB, $\mathrm{pH}$ 7.6) and finally with Tris buffered saline with Triton X-100 (TBST) $(0.5 \%[\mathrm{v} / \mathrm{v}] 100 \mathrm{X}$ triton in TBS; $\mathrm{pH}$ 7.6). Every washing step was done twice for 15 min each at RT. Afterwards, slices were incubated for 90 min with blocking buffer (as described in section 3.5.4). 


\section{MATERIALS AND METHODS}

Following the blocking step, tissue was incubated in $500 \mu \mathrm{l}$ blocking buffer containing primary antibodies for $48-72 \mathrm{hr}$ at $4^{\circ} \mathrm{C}$ on an orbital shaker. Subsequently, tissue was washed 4 times for 15 min each with TBST at RT. Then, tissue was incubated for $2 \mathrm{hr}$ in $500 \mu \mathrm{l}$ blocking buffer mixed with secondary antibodies at RT in complete darkness.

Following secondary antibody incubation, tissue was washed with TBST $(2 \times 15$ min) and with TBS ( 1 X $15 \mathrm{~min})$ at RT. If a DAPI staining was needed the tissue was incubated for 10 minutes in $500 \mu \mathrm{l}$ X PBS containing $10 \mu \mathrm{M}$ DAPI (Invitrogen) at RT in darkness. Tissue was then washed at RT once for 15 min with TBS and then two times for 15 min each with TB.

Brain slices were mounted on glass slides (Carl Roth) with a drop $(10 \mu \mathrm{l})$ of Fluoromount-G (Sigma) and covered with $12 \mathrm{~mm}$ glass coverslips (Thermo Scientific). Coverslips were sealed using transparent nail polish and imaged by confocal microscopy (section 3.6).

\subsection{MICROSCOPY: IMAGE ACQUISITION AND ANALYSIS}

\subsubsection{Epifluorescence microscopy}

Immunostained dissociated hippocampal neuronal cultures (see section 3.5.4) on glass coverslips were imaged using an upright epifluorescence Zeiss Axiovert.A1 microscope (Carl Zeiss). Images were acquired with a 100X/1.4 oil PlanApochromat objective (Carl Zeiss) (for ICC of synaptic vesicle markers) or a $63 X / 1.4$ oil Plan-Apochromat objective (Carl Zeiss) (for ICC of somatic protein expression). Images were captured with the Retiga-SRV camera (QImaging) and QCapture software (Qlmaging) and saved as 8-bit tiff files (RGB mode). Three to ten pictures of different neurons/regions of a given condition within the same 


\section{MATERIALS AND METHODS}

experiment were acquired using identical settings for image size, light intensity, gain and exposure time.

\subsubsection{Confocal microscopy}

Confocal microscopy was conducted using a Zeiss LSM 710 confocal microscope (Carl Zeiss). Acute hippocampal slices (see section 3.5.5) and ICC of synaptic markers of hippocampal neurons in vitro were imaged with a 40X Plan-Neofluar oil immersion objective (for single frame image acquisition) and 20X Plan-Apochromat air objective (for tiled image acquisition of multiple frames, e.g. whole hippocampus). A diode laser was used to excite the Alexa 405 fluorophore (excitation-emission: 401-421 nm) and DAPI (emission: 410-495 nm). The Alexa 488 fluorophore (excitation-emission: $493-520 \mathrm{~nm}$ ) was excited with a $488 \mathrm{~nm}$ argon laser. The Alexa 546 fluorophore was excited with a DPSSL laser (excitation-emission: 556-573 nm) and the Alexa 647 fluorophore was excited with a HeNe laser (excitation-emission: 650-665 nm).

A minimum of three images of hippocampal slices or neurons within the same experiment were acquired with identical frame size, scan speed, zoom, laser intensity, master gain, pinhole airy units, digital offset and digital gain. Images were captured and saved using the ZEN 2009 software and exported as 8 bit RGB tiff files.

\subsubsection{Immunocytochemistry analysis}

\subsubsection{Synaptic strength quantitation}

Vesicular transporters are proteins in synaptic vesicle membranes that pump neurotransmitters into synaptic vesicles (Johnson et al., 2003). The vesicular glutamate transporter 1 (vGluT1) Bellocchio et al., 2000; Takamori et al., 2000) and 


\section{MATERIALS AND METHODS}

the vesicular GABA/ glycine transporter (vGAT) (Mclntire et al., 1997) transfer cytoplasmic glutamate and GABA into synaptic vesicles, respectively, and are commonly used to mark synaptic vesicles at excitatory and inhibitory synapses, respectively.

In order to optically measure presynaptic modulations in synaptic strength of hippocampal neurons in vitro we conducted ICC co-staining for syt1 uptake (described in section 3.5.3) to mark recycling synaptic vesicles and vGluT1 (to mark synaptic vesicles at excitatory presynaptic terminals) or vGAT (to mark synaptic vesicles at inhibitory presynaptic terminals). The intensity of vGluT1 or vGAT signal corresponds to the number of synaptic vesicles per terminal and, in conjunction with syt1 uptake, gives a readout of presynaptic strength.

A mask of transfected cells was created using Adobe Photoshop CS5 (Adobe Systems Incorporated). All synaptic puncta positive for vGlut1 or vGAT (on a single transfected neuron identified by GFP or RFP fluorescence or in a neuronal network) were selected using OpenView 3.0 software (provided by Noam Ziv, Technion, Israel Institute of Technology, Haifa, Israel). The fluorescent intensity of vGluT1 or vGAT signal was measured using the "place area over puncta" function; threshold and delta intensity were configured for an accurate selection of synapses. Fluorescence intensity of puncta within the transfected neuron mask reported presynaptic strength on the selected neuron "on neuron" and fluorescence intensity of puncta outside of this mask reported presynaptic strength in surrounding "off neurons". The corresponding fluorescent intensity of the costained syt 1 taken up by recycling synaptic vesicles within selected areas was also measured. Blurry regions, puncta on somas and puncta not overlapping a visible neuronal process were excluded from analysis.

The values for fluorescent intensity generated by OpenView 3.0 were imported into Microsoft Excel 2010 (Microsoft). The fluorescence intensities in different conditions (transfections, treatments, cell type) within a single experiment (a 


\section{MATERIALS AND METHODS}

neuronal cell preparation in which ICC for all conditions was performed using the same antibody mix) were normalized to a range from 1-100 where 1 corresponded to the lowest and 100 to the highest fluorescence intensity detected in the experiment using the formula:

$$
x=\frac{i-\min }{\max -\min } \times 100
$$

Where $x=$ normalized value, $i=$ intensity value calculated by OpenView 3.0, $\min =$ lowest intensity value of the experiment and $\max =$ highest intensity value of the experiment. Since every experiment had slightly different cell density, antibody mix, microscope configurations, etc. this normalization allowed us to combine data from different experiments for comparison of conditions.

The fluorescence intensity of puncta in every image was averaged and the mean of the average fluorescent intensity across all experiments for multiple images was calculated for each condition. Pooled data of all images for every condition was plotted with the standard error of the mean, and Student's unpaired t-tests with Welch's correction were conducted to determine statistical significance using Prism 6 software (GraphPad).

\subsubsection{Excitatory and Inhibitory synapse number quantitation}

Synapse number analysis was performed by visual selection and counting of puncta positive for vGluT1 or vGAT immunofluorescence overlapping EGFP or MAP2 (a marker for dendrites of mature neurons (Kosik and Finch, 1987)). Synapses were counted along randomly selected 20-50 $\mu \mathrm{m}$ proximal dendritic processes.

Since high expressing TRPV1 neurons possess a very dense excitatory and inhibitory synaptic innervation where synapses are tightly packed (making the identification of individual synapses difficult), MetaMorph (Molecular Devices) 


\section{MATERIALS AND METHODS}

software was used to calculate the number of synapses contacting these neurons. The fluorescent signal of vGlut1 and vGAT positive immunostainings was selected by thresholding the signal (using the "set color threshold" function) to a specific level where only synapses were selected. Then, the "threshold" intensity value of a randomly selected $30-50 \mu \mathrm{m}$ area of a proximal dendrite (as explained above) was used to calculate the number of synapses in this area using the following formula:

$$
n=\frac{i / d}{l / p}
$$

Where $n=$ number synapses, $i=$ average fluorescence intensity in a selected thresholded area, $d$ = average area of a single synapse (in pixels), $I=$ length of the sample area (in pixels), and $p=$ number of pixels per micron.

Synapse number for every condition was then normalized to control conditions (EGFP transfected, or untreated condition). The normalized synapse number of every image was averaged and the mean of the average synapse number across all experiments was calculated for each condition. Pooled data from all images for every condition was plotted with the standard error of the mean and Student's unpaired t-tests with Welch's correction were conducted to determine statistical significance using Prism 6 software (GraphPad).

\subsubsection{Somatic protein expression}

In order to assess the somatic expression level of specific proteins (e.g. TRPV1, BDNF, NGF, etc.), the average fluorescence intensity in regions placed around somas was measured using the ImageJ or MetaMorph software. Fluorescence intensity in somas of each image was averaged and normalized to control. The mean of the average intensity of soma fluorescence intensity across all experiments was calculated for each condition. Pooled data of all images for every condition was plotted with the standard error of the mean and Student unpaired t- 


\section{MATERIALS AND METHODS}

tests with Welch's correction were conducted to determine statistical significance using Prism 6 software (GraphPad).

\subsubsection{Quantitation of neuronal marker co-localization}

Quantitation of percent co-localization of high TRPV1-expressing neurons with different neuronal markers was performed by identifying cells expressing TRPV1, and then subsequently imaging the other co-immunostained marker, and vice versa - identifying cells expressing a marker and then imaging TRPV1 signal - in 5-7 images each per experiment. The total percent of neurons with TRPV1 signal, neuronal marker signal, or both was plotted in Excel (Microsoft) or Prism 6 (GraphPad) software.

\subsection{NEUROPHARMACOLOGICAL ASSAYS}

To examine the effects of TRPV1 channel activity in excitatory and inhibitory synaptic plasticity $50 \mathrm{nM}$ or $1 \mu \mathrm{M}$ capsaicin (Sigma-Aldrich) and $1 \mu \mathrm{M}$ SB-366791 (Sigma-Aldrich) (Gunthorpe et al., 2004) a selective TRPV1 antagonist were used individually or together.

Two drugs were used to increase neuronal activity in vitro: $10 \mu \mathrm{M}$ bicuculline (Tocris), a competitive antagonist of $\mathrm{GABA}_{A}$ receptors (Johnston, 2013; Sillito, 1975) and $50 \mu \mathrm{M}$ forskolin (Sigma-Aldrich), which increases intracellular cAMP levels (Shoback and Brown, 1984) and reduces capsaicin-induced desensitization of TRPV1 channels (Schnizler et al., 2008). Neuronal activity was blocked with 1 $\mu \mathrm{M}$ TTX (Tocris), which blocks voltage gated $\mathrm{Na}^{+}$channels and prevents the firing of action potentials (Hwang and Noguchi, 2007; Narahashi et al., 1964). To determine if NGF affects TRPV1 expression hippocampal cultures were incubated with $1 \mathrm{ng} / \mathrm{ml}$ 2.5S NGF (Promega). 
MATERIALS AND METHODS

All pharmacological treatments were for $21 \mathrm{hr}$. at DIV 2-4 (before synapse formation) or DIV 10 (after synapse formation). After treatment, cultures were fixed for immunocytochemistry or homogenized with 1X sterile DPBS (Sigma-Aldrich) for Western blots.

\subsection{ELECTROPHYSIOLOGY}

\subsubsection{Miniature excitatory and inhibitory postsynaptic current recordings}

Whole cell patch clamp recordings from cultured rat hippocampal neurons (growing on individual glass coverslips) were performed at RT at DIV 12-14 in a custommade recording chamber. Transfected neurons were visualized with an epifluorescence upright microscope Olympus BX51WI (Olympus) equipped with a fluorescent light source Lumen 200 Pro (Prior Scientific) and the adequate filters for GFP detection (needed to identify transfected neurons). The localization of the neurons was done using either a 10x/0.3 water UMPlanFL $N$ objective (Olympus) or a 40x/0.3 LUMPlanFL $N$ objective (Olympus). For mEPSC and mIPSC recordings the artificial cerebrospinal fluid (ACSF) extracellular solution contained (136 mM NaCl, $2.5 \mathrm{mM} \mathrm{KCl}, 10 \mathrm{mM}$ HEPES, $10 \mathrm{mM}$ D-glucose, $2 \mathrm{mM} \mathrm{CaCl}_{2}$ and $1.3 \mathrm{mM} \mathrm{MgCl} 2$; $\mathrm{pH}$ 7.4, adjusted with $10 \mathrm{M} \mathrm{NaOH}$; 290-300 mOsm; $1 \mu \mathrm{M}$ TTX (Tocris Bioscience)). ACSF was bubbled with carbogen $\left(95 \% \mathrm{O}_{2}\right.$ and $\left.5 \% \mathrm{CO}_{2}\right)$ to acidify it and achieve a $\mathrm{pH}$ of 7.2. For mEPSC recordings, the extracellular solution included $100 \mu \mathrm{M}$ picrotoxin (Tocris Bioscience); for mIPSC recordings, $10 \mu \mathrm{M}$ CNQX and $10 \mu \mathrm{M}$ AP-5 were added to the ACSF. The intracellular solution contained (130 mM K-gluconate, $10 \mathrm{mM} \mathrm{NaCl}, 1 \mathrm{mM}$ EGTA, $0.133 \mathrm{mM} \mathrm{CaCl} 2,2$ $\mathrm{mM} \mathrm{MgCl}$, $10 \mathrm{mM}$ HEPES, $3.5 \mathrm{mM}$ Na-ATP, $1 \mathrm{mM} \mathrm{Na-GTP;} \mathrm{pH=7.3;} 280 \mathrm{mOsm}$ ) for mEPSC detection and (136 mM CsCl, 10mM EGTA, $2 \mathrm{mM} \mathrm{CaCl}, 0 \mathrm{mM} \mathrm{MgCl}_{2}$, $10 \mathrm{mM}$ HEPES, $2 \mathrm{mM}$ Mg-ATP, $0.3 \mathrm{mM}$ Na-GTP; $\mathrm{pH}=7.3$, adjusted with $\mathrm{CsOH}$; 290-295 mOsm) for mIPSC detection. Recordings were performed with a HEKA 
MATERIALS AND METHODS

EPC10 USB double patch clamp amplifier coupled to Patchmaster acquisition software (HEKA Electronics Inc.). Borosilicate glass micropipettes (resistance of 2$5 \mathrm{M} \Omega$ ) used for the recording were pulled using a P-97 micropipette puller (Sutter Instruments). Recorded neurons were held in a voltage-clamp mode at $-60 \mathrm{mV}$ for both mEPSC and mIPSC recordings. Data was filtered using a low pass Bessel filter at $2.9 \mathrm{kHz}$ and digitalized at $5 \mathrm{kHz}$. Recorded traces were analyzed using Mini Analysis v6.0.3 software (Synaptosoft). The amplitude threshold for detection of mEPSCs was approximately $3.5 \times$ RMS noise, i.e. 14 pA and for mIPSCs was 20 pA. Only undisturbed traces of at least 1 min were considered for the analysis

Average amplitude and frequency were then plotted with standard error of the mean, and statistical significance was calculated using unpaired, non-parametric Welch's tests in Prism 6 software (GraphPad).

\subsubsection{Electrophysiological recording from hippocampal slices}

\subsubsection{Slice preparation}

Acute hippocampal slices were prepared from 8-10 week old B6/J and TRPV1 KO adult mice as previously described (Ramachandran et al., 2015a). Animals were anesthetized with isoflurane and decapitated. The brain was removed from the skull and hippocampus was dissected. Transverse $400 \mu \mathrm{m}$ thick hippocampal slices were obtained using a tissue chopper (Stoelting). Slices were collected in ice-cold artificial cerebrospinal fluid (ACSF) (124 mM NaCl, $4.9 \mathrm{mM} \mathrm{KCl}, 1.2 \mathrm{mM}$ $\mathrm{KH}_{2} \mathrm{PO}_{4}, 2.0 \mathrm{mM} \mathrm{MgSO}_{4}, 2.0 \mathrm{mM} \mathrm{CaCl}$, $24.6 \mathrm{mM} \mathrm{NaHCO}_{3}, 10.0 \mathrm{mM}$ D-glucose; saturated with $95 \% \mathrm{O}_{2}$ and $5 \% \mathrm{CO}_{2} ; \mathrm{pH} 7.4$ and $305 \mathrm{mOsm}$ ). 
MATERIALS AND METHODS

\subsubsection{Field stimulation post-synaptic potential recordings}

Hippocampal slices were incubated for $3 \mathrm{~h}$ at $32{ }^{\circ} \mathrm{C}$ and high oxygen tension (by bubbling with $95 \% \mathrm{O}_{2}$ and $5 \% \mathrm{CO}_{2} ; 30 \mathrm{l} / \mathrm{h}$ ) in an interface chamber containing $2 \mathrm{ml}$ ACSF. A constant flow $(0.76 \mathrm{ml} / \mathrm{min})$ of ACSF was perfused continuously during the incubation. For recording and stimulation of the field excitatory post-synaptic potential (fEPSP), monopolar lacquer-coated stainless steel electrodes (571000, A$M$ Systems) were positioned in the CA1 region (Figure 3 ). The fEPSP was recorded using a differential AC amplifier (Model 1700, A-M Systems) and a power (1401) analog to digital converter (Cambridge Electronic Design) and tracked using the PWIN software (IFN Magdeburg). For each input, a determination of the "test stimulation strength" was stipulated as the current necessary to cause a fEPSP of $40 \%$ of the maximal slope. $30 \mathrm{~min}$ after incubation with ACSF, the baseline recording was started with test stimuli consisting of 4 biphasic pulses of constant current $(0.2 \mathrm{~Hz}, 0.1 \mathrm{~ms} /$ polarity) every $5 \mathrm{~min}$ for at least $30 \mathrm{~min}$. Long-term potentiation (LTP) was induced with one (1XTET) strong tetanization protocol consisting of 100 biphasic constant-current pulses at $100 \mathrm{~Hz}$ and $0.2 \mathrm{~ms} /$ polarity (1XTET) with an intertrain interval of $5 \mathrm{~min}$. For the nicotine-dependent LTP experiments, $5 \mu \mathrm{M}$ nicotine was applied $30 \mathrm{~min}$ prior to LTP induction and until the end of the recording $(1 \mathrm{~h})$.

Long term depression (LTD) was induced with a low-frequency stimulus protocol of 900 bursts, where each burst consisted of three stimuli at $20 \mathrm{~Hz}$, with an interburst interval of $1 \mathrm{~s}$ and a stimulus duration of $0.2 \mathrm{~ms}$ per half-wave, for a total of 2700 stimuli at $1 \mathrm{~Hz}$ (Ramachandran et al., 2015b) Test stimuli were given 1, 3, 5, 11, $15,21,25$ and $30 \mathrm{~min}$ after the TET train and every $5 \mathrm{~min}$ thereafter for a maximum of $1 \mathrm{~h}$. 


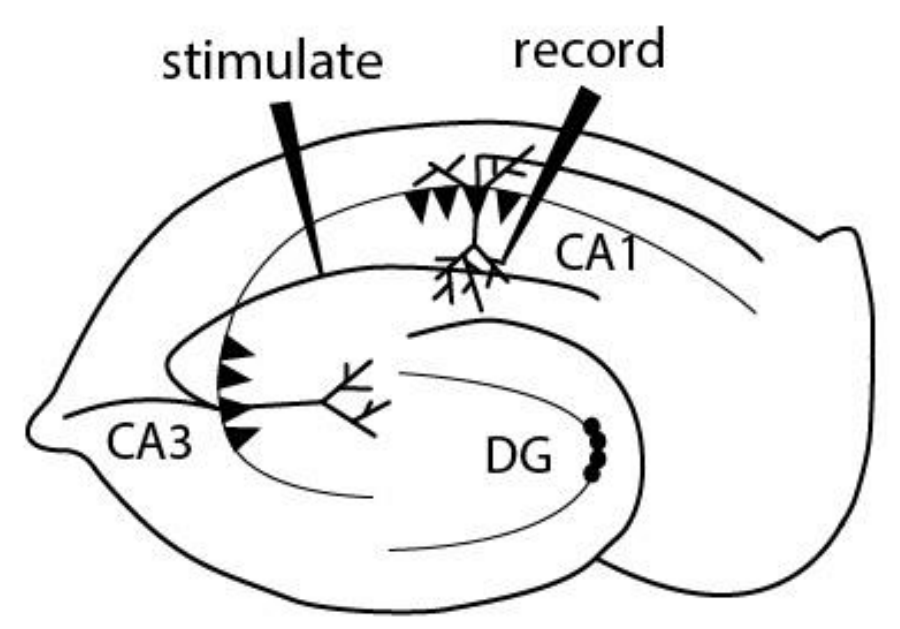

Figure 3. fEPSP recording protocol

Illustration of the position of stimulating and recording electrodes during LTP and LTD experiments performed in the hippocampal Schaffer collateral pathway.

\section{9. $\mathrm{CA}^{2+}$ IMAGING}

To test if capsaicin activates hippocampal neurons in vitro we used the Fluo-4 (Gee et al., 2000) (Invitrogen) calcium indicator at a concentration of 1:1000 added to DIV 13-14 mouse hippocampal neurons. After 10-20 minutes of incubation, coverslips were placed on a field stimulation imaging chamber (Warner Instruments) in $100-150 \mu \mathrm{l}$ of base solution $(140 \mathrm{mM} \mathrm{NaCl}, 5 \mathrm{mM} \mathrm{KCl}, 2 \mathrm{mM}$ $\mathrm{CaCl}$, $2 \mathrm{mM} \mathrm{MgCl}$, $5.5 \mathrm{mM}$ glucose, $20 \mathrm{mM}$ Hepes; $\mathrm{pH}=7$ ). The stimulation chamber was then connected to a biphasic stimulator and positioned on a Zeiss Axio Observer A1 epifluorescence microscope (Carl Zeiss) with a DG-4 wavelength switcher. A 40x/1.4 air Plan-Apochromat objective and a $488 \mathrm{~nm}$ wavelength filter was used to detect Fluo-4 fluorescence (excitation-emission: 494$516 \mathrm{~nm}$ ) Three-minute time series of 1 frame per second were acquired with an exposure time $\leq 200 \mathrm{~ms}$ using an Evolve Camera (Photometrics) controlled by MetaMorph software (Molecular Devices). After $1 \mathrm{~min}$ baseline recording, $301 \mathrm{~ms}$ $+10 \mathrm{~V}$ pulses at $40 \mathrm{~Hz}$ were delivered by field stimulation. Following field stimulation (at frame 120) a final concentration of $1 \mu \mathrm{M}$ capsaicin (or corresponding 


\section{MATERIALS AND METHODS}

volume of DMSO) was added by pipette and samples were imaged for an additional minute.

For analysis of intracellular $\mathrm{Ca}^{2+}$ responses to capsaicin, only somas of neurons responding to field stimulation were selected with the region tool in MetaMorph. Background (the average intensity of 5 regions without visible neuronal processes or cell bodies) was subtracted and the average intensity in cell somas throughout the time course was determined in Metamorph and further analyzed using a custom written script in Matlab. Frames were clipped from individual traces such that time of field stimulation and time of addition of capsaicin or DMSO in each trace was aligned with respect to each other. Fluorescence averaged over first 50 frames was taken as baseline $\left(F_{0}\right)$. The $\Delta F / F_{0}$ traces were calculated as $\left(F-F_{0}\right) / F_{0}$, where $F$ is the Fluorescence at time $(t)$. Cells that had $<20 \%$ increase upon field stimulation were excluded from the analyses. From the cells reacted to field stimulation, only those that showed a $>20 \%$ increase upon capsaicin addition were considered as responsive.

\subsection{RNA ISOLATION AND RT-PCR}

To detect if TRPV1 and its isoforms were expressed in hippocampus, brain, cerebellum and dorsal root ganglia (DRG), RNA was isolated from 2 to 3-month-old WT (B6/J) and TRPV1 KO mice. Animals were euthanized with $\mathrm{CO}_{2}$ and then decapitated and brains were collected in a $10-\mathrm{cm}$ petri dish on ice containing sterile 1X PBS. Hippocampi and dorsal root ganglia were isolated and collected in DNase and RNase free Eppendorf tubes (Thermo Fisher), snap-frozen with liquid nitrogen and stored at $-80^{\circ} \mathrm{C}$. Tissue was dissected from each mouse using separate tools in order to avoid tissue contamination.

RNA was isolated using Trizol (Sigma-Aldrich). Hippocampi and DRGs were homogenized with $500 \mu \mathrm{l}$ or $250 \mu \mathrm{l}$ Trizol respectively and incubated at RT for 5 


\section{MATERIALS AND METHODS}

min. Subsequently $100 \mu \mathrm{l}$ (hippocampus) or $50 \mu \mathrm{l}$ (DRGs) chloroform was added and mixed vigorously by inverting the tube 15 times and then allowing solution to settle for $3 \mathrm{~min}$ at RT. Samples were then centrifuged for $15 \mathrm{~min}$ at $4^{\circ} \mathrm{C}$ at 13,000 rcf. The upper aqueous supernatant was transferred to a new micro-centrifuge tube, additional isopropanol was added ( $250 \mu \mathrm{l}$ for hippocampus and $125 \mu \mathrm{l}$ for DRGs) and mixed by gentle inversion followed by a $-20^{\circ} \mathrm{C}$ incubation for $1 \mathrm{hr}$. Samples were then centrifuged at $13,000 \mathrm{rcf}$ for $30 \mathrm{~min}$ at $4^{\circ} \mathrm{C}$. The supernatant was discarded and the obtained RNA pellet was washed with $1 \mathrm{ml} 75 \%$ ice cold $70 \%$ ethanol. After washing, a final centrifugation step of 5 min at $4^{\circ} \mathrm{C}$ RT 13,000 rcf was performed, supernatant was discarded and the RNA pellet was air-dried at RT until the ethanol was completely evaporated. The RNA pellet was finally dissolved with DNase- and RNase-free water (30 $\mu \mathrm{l}$ for hippocampus and $20 \mu \mathrm{l}$ for DRGs). Samples were treated with DNase I (Ambion) to eliminate any remaining chromosomal DNA (Vanecko and Laskowski, 1961).

Purity and concentration of extracted RNA was determined using a spectrophotometer (NanoDrop ND-1000) with ND-1000 v3.5.2 software (peQLabBiotechnologie). Only isolated RNA with an integrity value of more than 8 was used.

cDNA was synthesized using $2 \mu \mathrm{g}$ (hippocampus) or $1 \mu \mathrm{g}$ (DRGs) of the isolated RNA. Dissolved RNA samples were diluted with DNase and RNase free water to a final volume of $11 \mu \mathrm{l}$ and mixed with $9 \mu \mathrm{l}$ of the cDNA synthesis reaction mix (see below) for a final volume of $20 \mu \mathrm{l}$. RNA was reversed transcribed with the Transcriptor High Fidelity cDNA synthesis kit (Roche Applied Science) under the following cycling conditions: 1 cycle of 10 minutes at $25^{\circ} \mathrm{C}, 1$ cycle of 30 minutes at $55^{\circ} \mathrm{C}$ and a final cycle of $5 \mathrm{~min}$ at $80^{\circ} \mathrm{C}$, followed by a hold at $4{ }^{\circ} \mathrm{C}$. A reverse transcription - polymerase chain reaction (RT-PCR) without reverse transcriptase served as a negative control. Isolated RNA and negative controls were stored at $20^{\circ} \mathrm{C}$. 


\section{MATERIALS AND METHODS}

Reaction mix for cDNA generation:

$4 \mu \mathrm{l}$

$0.5 \mu l$

$2 \mu \mathrm{l}$

$0.5 \mu \mathrm{l}$

$2 \mu \mathrm{l}$
Reaction Buffer, 5x concentrated (Roche Applied Science)

RNase inhibitor

Deoxynucleotides

Reverse transcriptase

Random hexamer primer

\subsubsection{Primer design, PCR and Q-PCR}

Quantitative Q-PCR was performed on a light cycler 480 system (Roche Applied Science) using the LC480 SYBR green Master mix (Roche Applied Science). $2 \mu \mathrm{l}$ of the synthesized cDNA library was used for Q-PCR with TRPV1 primers (see below) and 1:10 diluted cDNA for the reference control tubulin alpha-1B. All data obtained was normalized to the mRNA levels of tubulin alpha-1B. The Q-PCR reaction mix and cycling conditions for amplification were as follows:

Reaction mix for Q-PCR

$2 \mu \mathrm{l}$ CDNA

$0.75 \mu \mathrm{l}$ Forward primer $(10 \mu \mathrm{M})$

$0.75 \mu \mathrm{l}$ Reverse primer $(10 \mu \mathrm{M})$

$7.5 \mu \mathrm{l}$ LC480 Master mix, 2x concentrated

$4 \mu \mathrm{l}$ DNase- and RNase-free water

Q-PCR cycle conditions:

$\left.\begin{array}{ll}95^{\circ} \mathrm{C} & 5 \mathrm{~min} \\ 95^{\circ} \mathrm{C} & 10 \mathrm{sec} \\ 60^{\circ} \mathrm{C} & 15 \mathrm{sec} \\ 72^{\circ} \mathrm{C} & 10 \mathrm{sec} \\ 95^{\circ} \mathrm{C} & 15 \mathrm{sec} \\ 67^{\circ} \mathrm{C} & 30 \mathrm{sec}\end{array}\right]$




\section{MATERIALS AND METHODS}

$\begin{array}{ll}95^{\circ} \mathrm{C} & 15 \mathrm{sec} \\ 40^{\circ} \mathrm{C} & 10 \mathrm{sec} \\ 4^{\circ} \mathrm{C} & \text { hold }\end{array}$

Primers used for identification of expression of the TRPV1 channel pore region (deleted exon in the TRPV1 KO), the TRPV1 N-T and C-T, the TRPV1 channel splice isoforms vanilloid receptor 5' splice variant (VR.5'sv) and for the housekeeping gene Tubulin alpha-1B were either custom designed or as described previously (Table 3). All primers were synthesized by and purchased from SigmaAldrich®.

Table 3. List of primers for Q-PCR.

\begin{tabular}{|c|c|c|c|}
\hline Transcript & Forward primer (5'-3') & Reverse primer (5'-3') & Description \\
\hline TRPV1-N-1 & gagtgtgcctgcacctagc & tcagattcatccgagtctaagc & $\begin{array}{c}\text { TRPV1 N-Terminus } \\
\text { specific }\end{array}$ \\
\hline TRPV1 KOr & cttgtttggattttccacagc & cacttgtgtggtggggact & $\begin{array}{c}\text { Specific TRPV1 KO } \\
\text { deletion }\end{array}$ \\
\hline TRPV1-C & ggcatgtccctaggacttca & agctcgcttcccacacac & TRPV1 C-Terminus \\
\hline VR.5' sv-S & tgaccctcttggtggagaatgg & tgaggctagtccctggatgactg & VR.5'sv specific \\
\hline Tuba1B & aggagctggcaagcatgt & agctgctcaggctggaagag & Housekeeping gene \\
\hline
\end{tabular}

For VR.5' sv, the only published sequence to our knowledge was reported for rat (rattus norvegicus) tissue (Schumacher et al., 2000) (GenBank ${ }^{\mathrm{TM}}$ accession number AF158248.2). These authors designed primers to detect VR.5' sv or TRPV1 gene expression in rat tissue. We designed primers to detect the same region in mouse, by aligning the TRPV1 genomic mouse sequence (GenBank ${ }^{\mathrm{TM}}$ accession number NC_000077.6) with the rat TRPV1 genomic sequence (GenBank $^{\mathrm{TM}}$ accession number NC_005109.4) using the open source software "A plasmid editor" v2.0.47 (Copyright 2003-2009 by M. Wayne Davis) (ApE@). 


\section{MATERIALS AND METHODS}

For custom-designed primers for TRPV1 we obtained the mouse (Mus musculus) mRNA sequence of this gene from GenBank ${ }^{T M}$ of the National Center of Biotechnology Information, U.S. (NCBI) (http://www.ncbi.nlm.nih.gov/nuccore) under the GenBank ${ }^{\mathrm{TM}}$ accession number: NM_001001445.2. For VR.5' sv we obtained the coding sequence of the mouse gene as mentioned above. We then chose primers using the online tool "Universal Probe Library Assay Design Center" (Roche Diagnostics ${ }^{\mathrm{TM}}$ ) by entering the complete NCBI reference sequence or the specific exons we aimed to detect, such as those of amino and carboxyl termini, the pore region (to detect the expression of functional TRPV1 channels) and the unique VR.5' sv sequences, to detect this isoform.

\subsection{BIOCHEMICAL METHODS}

\subsubsection{Sample tissue collection and preparation}

For immunoblotting of hippocampal or HEK 293 cells grown on $10 \mathrm{~cm}$ tissue culture dishes (CellStar), the culture medium was removed, cells were washed once with sterile DPBS (Sigma-Aldrich) and scraped off the dish in $500 \mu \mathrm{l}$ sterile DPBS (Sigma Aldrich) using a cell scraper (Sarstedt) into an Eppendorf tube and snap-frozen in liquid nitrogen.

For preparation of whole brain and hippocampus homogenates, tissue was dissected from 2-4-month-old mice and collected in DNase and RNase free Eppendorf tubes and immediately frozen in liquid nitrogen. Tissue was homogenized in homogenization buffer (320 mM sucrose and 4 mM HEPES; $\mathrm{pH}$ 7.4, adjusted with $\mathrm{NaOH}$ ) made fresh right before use and kept at $4{ }^{\circ} \mathrm{C}$. Homogenization was done in $9 \mathrm{ml}$ of $4^{\circ} \mathrm{C}$ homogenization buffer (supplemented with protease inhibitors; 1:1000 dilution) using a glass-Teflon homogenizer with 810 strokes at $900 \mathrm{rpm}$. 


\subsubsection{Protein quantification}

Protein concentration in homogenized tissue was determined with a BCA protein assay kit (Novagen) (Brown et al., 1989; Kessler and Fanestil, 1986; Smith et al., 1985; Wiechelman et al., 1988). Absorbance was measured at $562 \mathrm{~nm}$ on a plate reader.

\subsubsection{Protein separation (SDS-PAGE)}

Protein separation was performed following the Schägger protocol (Schägger and von Jagow, 1987). Proteins were denatured: samples were mixed with the Schägger sample buffer (50 mM Tris $4 \%$ SDS, $0.01 \%$ Serva Blue G, $12 \%$ glycerol; pH 6.8, adjusted with $\mathrm{HCl}$; for reducing $2 \%$ mercaptoethanol) and heated at $99^{\circ} \mathrm{C}$ for $10 \mathrm{~min}$. Equal amounts of protein were loaded into each lane and proteins were separated by $10 \%$ [w/v] SDS-PAGE under constant current using a Bio-rad electrophoresis power supply.

\subsubsection{Western blotting}

Proteins were transferred from a polyacrylamide gel to a nitrocellulose membrane as previously described (Towbin et al., 1979) at $100 \mathrm{~mA}$ for $1 \mathrm{~h}$.

For immunoblotting, membranes were incubated for 1 hour at RT in WB blocking buffer $(5 \%[\mathrm{w} / \mathrm{v}]$ lyophilized non-fat milk in $1 \mathrm{X}$ TBST). Primary antibody (targeting the desired protein) (see Table 1) was then added to the WB blocking buffer. The membrane was incubated $\mathrm{ON}$ at $4^{\circ} \mathrm{C}$ in primary antibody solution. The membrane was subsequently washed 3 times with TBST and then incubated for $1 \mathrm{~h}$, at RT with HRP-conjugated secondary antibodies (see Table 2) in WB blocking buffer. After washing 3 times with TBST, the custom-made chemiluminescent substrate ECL consisting of $1 \mathrm{ml}$ of solution $\mathrm{A}$ (2.42 $\mathrm{g}$ of Tris- $\mathrm{HCl}, 500 \mathrm{mg}$ of luminol sodium 


\section{MATERIALS AND METHODS}

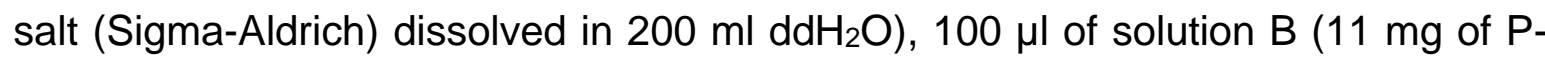
coumaric acid (Sigma-Aldrich) dissolved in $10 \mathrm{ml}$ DMSO) and $0.3 \mu \mathrm{l}$ of solution $\mathrm{C}$ (30-35\% [v/v] of $\mathrm{H}_{2} \mathrm{O}_{2}$ ) was poured to the membrane for at least $3 \mathrm{~min}$ and protected from daylight. For visualization, the signal was captured using a LAS reader (Fuji). The signal intensity was quantified using the open source software Image $\mathrm{J}$, and values were plotted in Excel (Microsoft). Significance was determined using Student's unpaired t-tests with Welch's correction in Prism 6 software (GraphPad).

\subsubsection{Isolation of synaptic vesicles from mouse brain homogenates}

This method was performed by Saheeb Ahmed, a fellow post-doctoral scientist in the Trans-synaptic signaling Group of the ENI-Göttingen, as previously described (Ahmed et al., 2013; Pavlos et al., 2010).

\subsubsection{Protease treatment of synaptosomes}

Synaptosomes were isolated as previously described (Fischer von Mollard et al., 1991). After Synaptosome purification, protease treatment was performed as described (Boyken et al., 2013). Immunoblots for detection of TRPV1 were done using the N-terminal TRPV1 antibody (Neuromab) (Table 1). 
RESULTS

\section{RESULTS}

\subsection{TRPV1 EXPRESSION IN BRAIN AND HIPPOCAMPUS}

Since the generation of a TRPV1 KO mouse line in the year 2000 (Caterina et al., 2000) this mutant animal has become a model for understanding the mechanism underlying the process of nociception. Intensive research has been conducted in order to identify in which neural tissue TRPV1 is expressed in the peripheral and central nervous system. To date the expression profile of this channel in the brain is still unclear; especially in hippocampus, the existence of the TRPV1 channel is under debate.

We started this research with the basic question: is TRPV1 expressed in brain, and hippocampus tissue? In order to assess if TRPV1 expression occurs in both brain and hippocampus we conducted biochemical and immunological assays in different neural tissues from WT and TRPV1 KO mouse lines.

First, we ensured that the deletion of the TRPV1 gene resulting in loss of function was present in TRPV1 KO animals used for experimentation. WT primer pair for TRPV1 amplified a sequence of 984 bp only from isolated genomic DNA of WT animals, whereas the TRPV1-mutant primer pairs amplified the expected $450 \mathrm{bp}$ sequence only from TRPV1 KO genomic DNA (data not shown). 


\section{RESULTS}

\subsubsection{TRPV1 and VR.5' sv mRNA are differentially expressed in the hippocampus and DRG from WT and TRPVI $\mathrm{KO}$ mice}

We further assessed the expression at the mRNA level of TRPV1 and the TRPV1 $\mathrm{N}-\mathrm{T}$ alternative splice isoform (VR.5' sv) in the hippocampus and DRG (used as a positive control for the expression of both isoforms) of 2-3-month-old adult mice. We confirmed the presence of both TRPV1 and VR.5' sv transcripts in both DRG and hippocampus (Figure $4 \mathrm{~A}-\mathrm{C}$ ).

As expected, primers designed to amplify part of the sequence that is deleted in the TRPV1 KO mouse model (TRPV1 KOr) corresponding to exon 13 (encoding part of the fifth and all of the sixth transmembrane domain, including the putative pore region) are absent in both DRG and hippocampus from TRPV1 KO animals (Figure $4 \mathrm{~A}-\mathrm{C}$ ), whereas in WT animals amplification of this sequence was detected in both tissues. Although in the hippocampus the expression was minimal it was still amplified in WT mice, contrasting with the negligible amplification observed in TRPV1 KO samples (Figure $4 \mathrm{C}$ ).

Similar to the results observed with the primer pair TRPV1 KOr, the amplification of specific nucleotide sequences of the amino-end of TRPV1 mRNA using the primer pair TRPV1-N1 (encoding a sequence stretch from exon 1 and 2) showed that in WT animals TRPV1 mRNA levels are higher in DRG compared to the hippocampus (Figure $4 \mathrm{~A}$ ). While in TRPV1 $\mathrm{KO}$ animals the amplification of this sequence was almost absent in DRG (Figure $4 \mathrm{~B}$ ); in the hippocampus, the expression did not significantly change in TRPV1 KOs compared to WT.

We also designed primers that amplify the putative region of exon 16 encoding for the carboxyl-terminus amino acid sequence recognized by the TRPV1 C-T antibody (Millipore). Similar to the levels of amplification observed by both the TRPV1 KOr and the TRPV1-N1 primer pairs, the expression of the C-T mRNA 


\section{RESULTS}

sequence was significantly higher in DRG than in hippocampus of WT animals (Figure $4 \mathrm{~A}$ ) and in TRPV1 KO DRG the mRNA was significantly less than in WT (Figure $4 \mathrm{~B}$ ). Surprisingly (unlike TRPV1 KOr and TRPV1-N1 primers) in the hippocampus the levels of mRNA corresponding to the C-T of TRPV1 was increased by 350 times compared to WT (Figure $4 \mathrm{C}$ ).

This tremendous increase in the levels of the C-T sequence could mean that a TRPV1 splice isoform is compensating for the absence of TRPV1 in the knockouts. We therefore further tested the expression of the TRPV1 alternative N-T splice isoform (i.e. VR.5' sv) known to lack most of the N-T but conserving the entire C-T. We designed VR.5' sv mouse specific primers modified from previous studies in rat (Schumacher et al., 2000). These primers amplify one part of a VR.5' sv-specific sequence (in exon 1 of VR.5' sv) that is not present in TRPV1. As shown in (Figure $4 \mathrm{~A}$ ), our results show that in DRG this isoform is expressed (like TRPV1) at higher levels compared to hippocampus, and in TRPV1 KO DRG these levels are significantly reduced (Figure $4 \mathrm{~B}$ ) Interestingly, in contrast to DRG, the level of amplification of the VR.5' sv-specific stretch in hippocampus is significantly increased in TRPV1 KOs compared to WT.

We further compared the relative levels of mRNA of TRPV1 (using the abovementioned primer pairs) and VR.5' sv in either the DRGs or the hippocampus of adult mice. As depicted in Figure 5A our analyses show that in DRGs the mRNA levels for primer pairs amplifying either the pore region or the amino terminus were approximately 15 - 16 times higher than those of VR.5' sv. Expression of the C-T TRPV1 mRNA stretch was 44 times higher than that of VR.5' sv. Interestingly the mRNA levels of the C-T region of TRPV1 were significantly higher than those of the $\mathrm{N}-\mathrm{T}$ and pore region (the latter two did not differ in amount).

Opposite to the transcription profile in DRGs, in the hippocampus mRNA levels of the VR.5' sv were 10-14 times higher than either the pore region or the N-T of the TRPV1 channel. Intriguingly the amount of C-T TRPV1 mRNA was double that of 
VR.5' sv. Similar to DRGs, mRNA levels of TRPV1 C-T were also higher than the TRPV1 pore region and TRPV1 N-T mRNA levels in hippocampus (Figure 5 B).

A

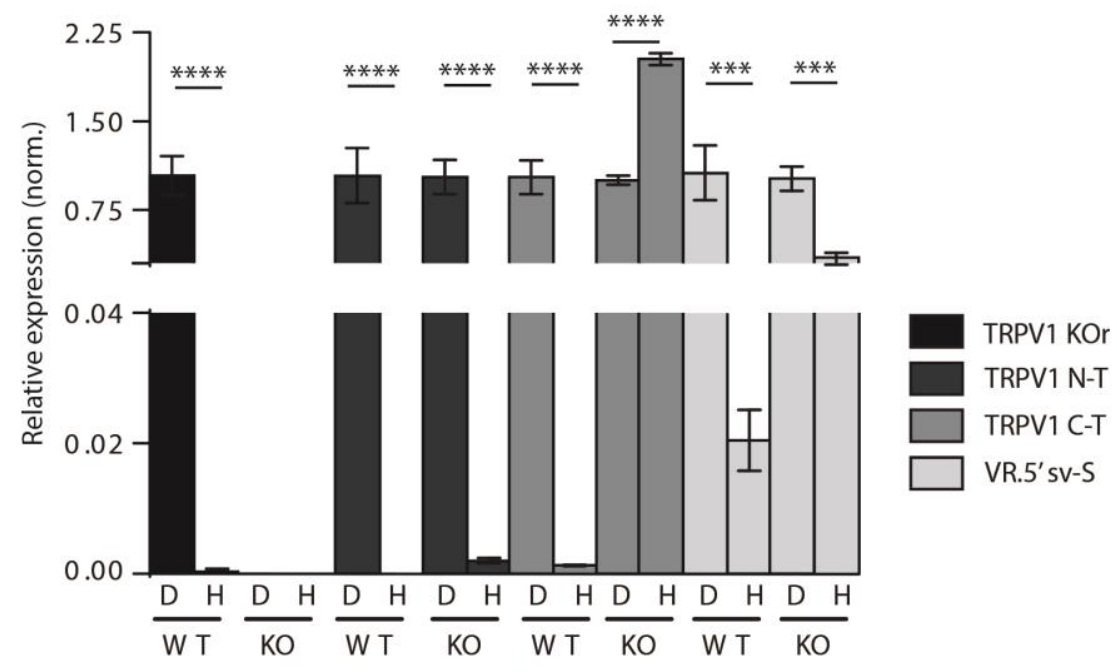

B

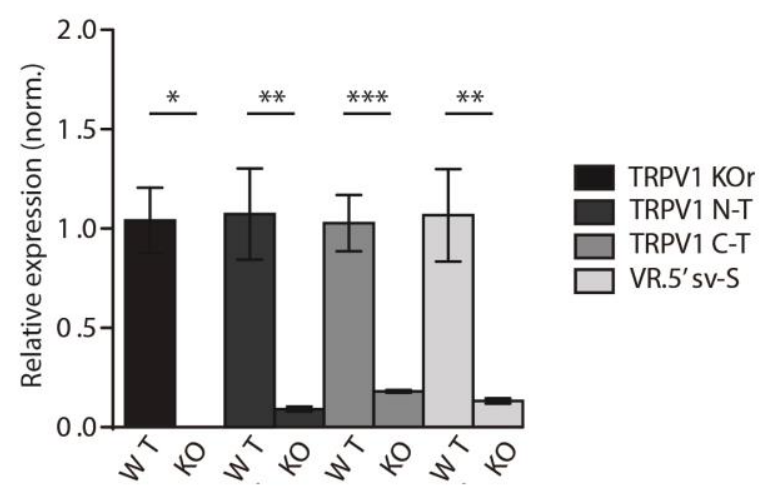

C

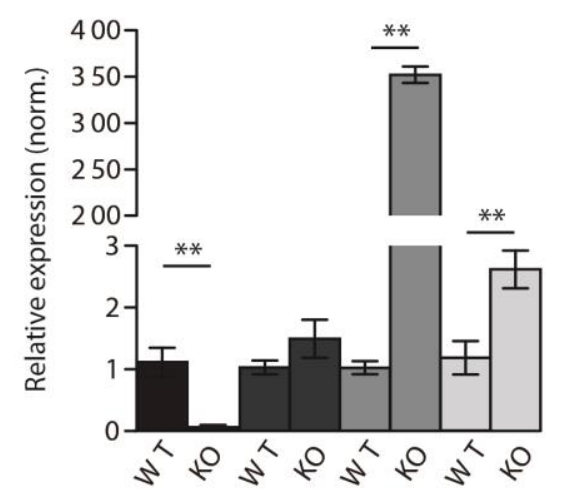

Figure 4. Differential mRNA expression of specific TRPV1 domains and VR.5' $\mathrm{sV}$ in the hippocampus and DRG from WT and TRPV1 KO mice. A) Q-PCR showing the relative mRNA expression levels of the TRPV1 N-T, C-T and pore regions, and of VR.5' sv, amplified by specific primer pairs in DRG (D) and hippocampus $(H)$ normalized to DRG levels. In the hippocampus levels of all TRPV1 sequences are significantly less than in DRG except for TRPV1 C-T and VR.5' sv in TRPV1 KOs. B) Q-PCR values for DRG samples of WT and TRPV1 KO mice normalized to WT levels. mRNA levels of all amplified sequences are significantly reduced in TRPV1 KO DRGs compared to WT. C) Q-PCR values for hippocampus samples of WT and TRPV1 KO mice normalized to WT levels. mRNA level of the pore domain is absent in TRPV1 KO hippocampus; the C-T of TRPV1 and the VR.5' sv are significantly increased in TRPV1 KO hippocampus. $(n=3$ each for WT and TRPV1 KO samples of hippocampus and DRG; error = SEM, significance was determined by unpaired Student's t-test $\left.{ }^{*} p<0.05,{ }^{* *} p<0.01,{ }^{* * *} p<0.001,{ }^{* * *} p<0.0001\right)$. Q-PCR done by Dr. Rashi Halder. 
A

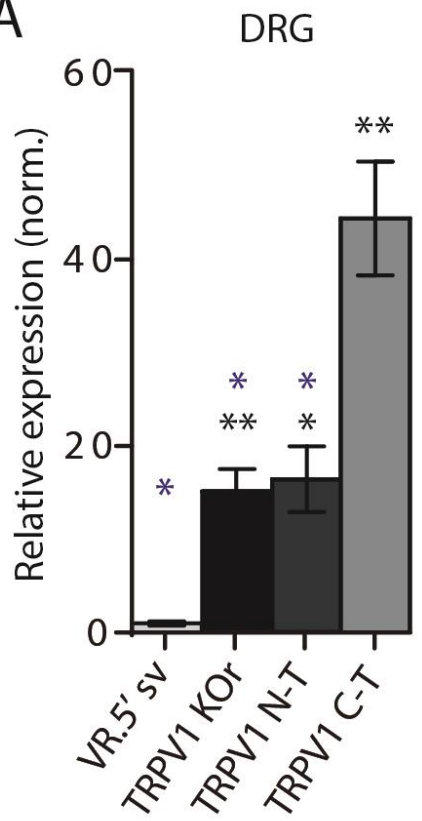

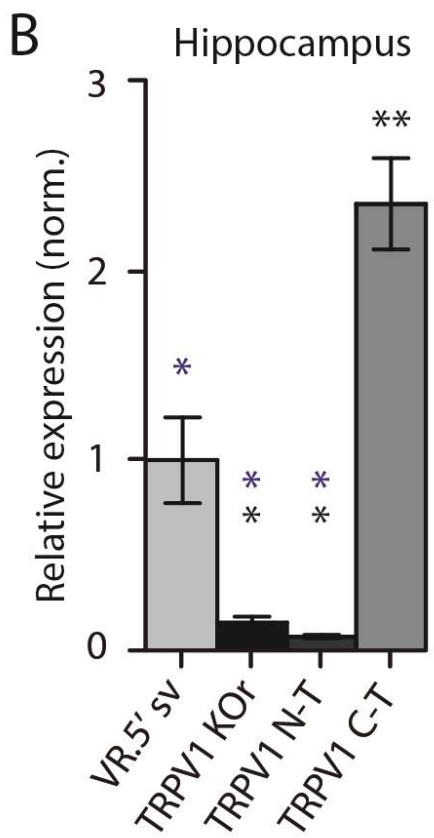

Figure 5. Differential mRNA expression of specific TRPV1 domains and VR.5' sv in the DRG and hippocampus from WT mice. A) Q-PCR showing the relative mRNA expression levels of the TRPV1 N-T, C-T and pore regions compared to VR.5' sv in DRG samples. Levels of TRPV1 N-T and KOr sequences are significantly higher than for VR.5' sv, and TRPV1 C-T levels are significantly higher than for $\mathrm{N}-\mathrm{T}$ or KOr sequences. B) Q-PCR showing the relative mRNA expression levels of the TRPV1 N-T, C-T and pore regions compared to VR.5' sv in adult hippocampi. Levels of the N-T and KOr sequences are significantly lower than those of VR.5' sv. TRPV1 C-T is expressed at the highest levels. For A and B values of mRNA expression were normalized to VR.5' sv values ( $n=3$ each for WT hippocampus and DRG; error = SEM, significance was determined by unpaired Student's t-test, ${ }^{*} p<0.05$, ${ }^{* \star} p<0.01$, ${ }^{* \star *} p<0.001,{ }^{* * * *} p<0.0001$; black asterisks indicate significant difference compared to VR.5' sv mRNA levels; blue asterisks indicate significant difference compared to TRPV1 C-T mRNA levels. Q-PCR done by Dr. Rashi Halder.

\subsubsection{TRPV1 protein expression levels in the mouse brain and hippocampus}

In order to assess if the TRPV1 channel is expressed in brain and hippocampus at the protein level, WB were conducted using an N-terminus specific TRPV1 antibody (NeuroMab) in whole brain and hippocampus homogenates. The molecular weight of TRPV1 is $94.976 \mathrm{kD}$, where the expected band size is approximately $90 \mathrm{kD}$. To test the specificity of this antibody for TRPV1 we did WB 
of TRPV1-transfected HEK cells in vitro; as shown in (Figure $6 \mathrm{~B}$ ) the $90 \mathrm{kD}$ band corresponding to TRPV1 was detected only in transfected cells.
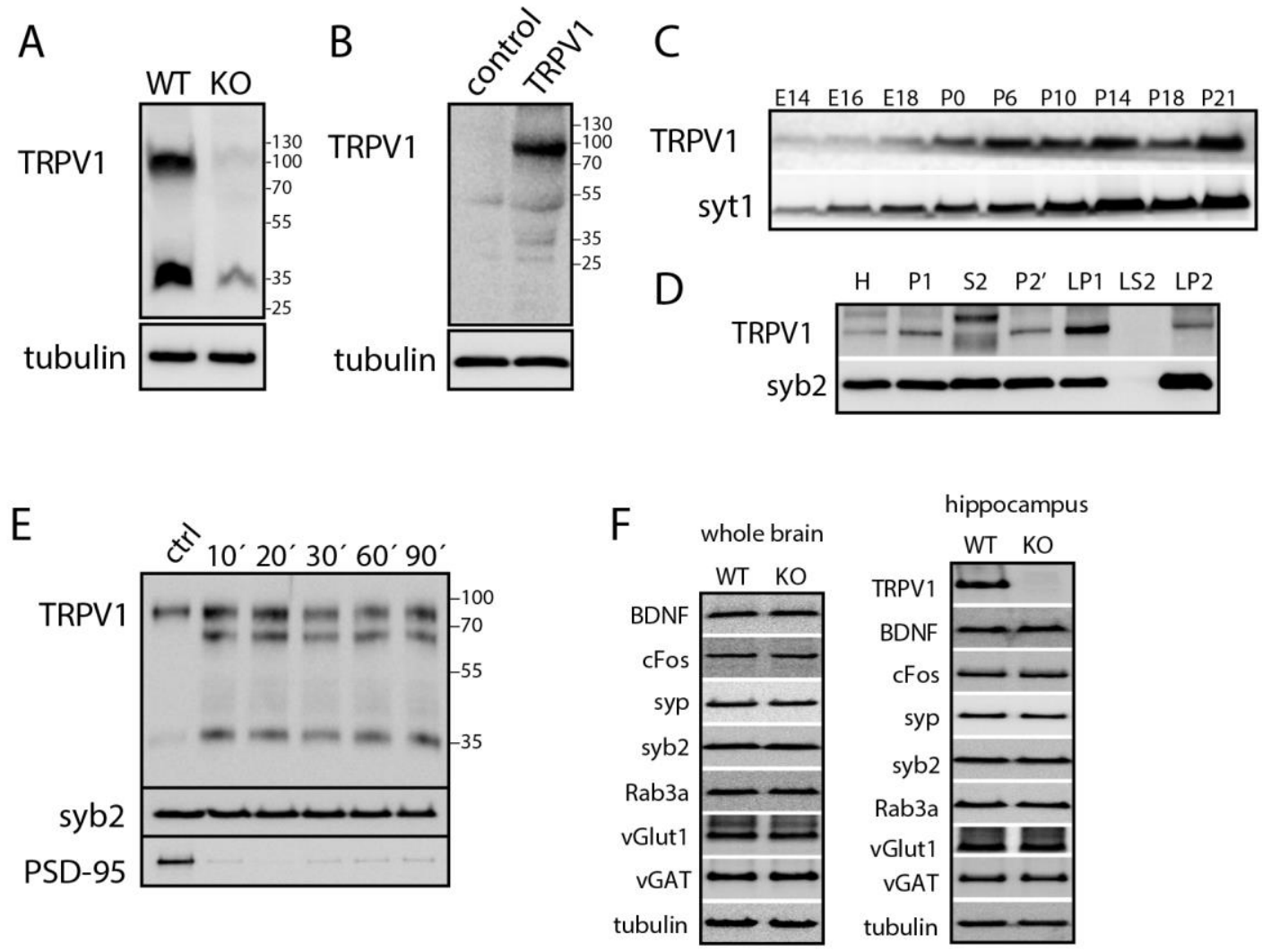

Figure 6. TRPV1 expression in mouse brain and hippocampus. A) Western blot confirms that the N-T TRPV1 antibody (from NeuroMab) recognizes a specific band at the expected size for TRPV1 in WT but not in TRPV1 KO brain homogenates. Tubulin was used as a loading control; $n=3$ repetitions. B) Western blot showing that the N-T TRPV1 antibody recognizes a band of the correct size in homogenates of HEK cells transfected the TRPV1-EGFP construct, but not in control untransfected cells; tubulin was used as a loading control. C) Western blot showing the expression profile of TRPV1 in brain homogenates during development. The onset of TRPV1 expression is late embryonic / early post-natal and persists during adulthood. Syt1 expression, which increases throughout development and remains constant in adulthood, is shown for comparison. D) Subcellular fractionation shows that TRPV1 expression is mainly present in plasma membrane fractions. (indicated fractions are homogenate $(\mathrm{H})$, nuclear and large membrane pellets (P1), synaptosomal supernatant (S2), synaptosomes (P2'), presynaptic plasma membrane (LP1), synaptic cytosol (LS2), and crude synaptic vesicles (LP2)). E) Trypsin cleavage assay in purified synaptosomes $(n=3)$. The TRPV1 antibody detected the full-length $90 \mathrm{kD}$ band, but also a $65 \mathrm{kD}$ cleavage product. The pre-synaptic marker syb2 was only detected as a full-length protein (18 kD). The post-synaptic marker PSD95 full-length protein (105 kD) was not detected following trypsin treatment. F) Western blot of synaptic proteins in whole brain (left) and hippocampus (right) from WT and TRPV1 KO mice. TRPV1 is expressed in WT but not in TRPV1 KO hippocampus; tubulin serves as a loading control. Western blots, subcellular fractionation and trypsin cleavage assays conducted by Dr. Saheeb Ahmed. 


\section{RESULTS}

Next, we surveyed the expression profile of TRPV1 during neural development from embryonic stages to early adulthood in WT mice. As depicted in (Figure $6 \mathrm{C}$ ) we found that the expression of TRPV1 and syt1 (used as a control synaptic protein) was already present at embryonic day 14 (E14), E18 and postnatal day 0 (P0). After birth the expression of TRPV1 and syt1 increased and the expression persisted until at least 2-3 months into adulthood.

Brain and hippocampus homogenates from 2-3-month-old WT and TRPV1 KO mice allowed us to confirm that the observed band in fact corresponded to the TRPV1 protein. As shown in (Figure $6 \mathrm{~A}, \mathrm{~F}$ ) the expected $90 \mathrm{kD}$ band was observed in both brain and hippocampus tissue homogenates of WT mice but not in TRPV1 KOs. A lower molecular weight band ( $35 \mathrm{kD})$ was also consistently detected in most of the WB using the N-T TRPV1 antibody; the intensity of this band was always lower than the TRPV1-specific one. We also tested the expression levels of other synaptic proteins, neurotrophins (i.e. BDNF) and cytoskeletal proteins. We did not find any significant change in these proteins in TRPV1 KO tissue compared to WT (expected band sizes shown) (Figure 6 F).

\subsubsection{Pre- and post-synaptic expression of TRPV1}

After detecting TRPV1 protein expression in the adult mouse brain and hippocampus we were keen to clarify if TRPV1 is expressed synaptically.

One way to determine if TRPV1 is expressed synaptically is by subcellular fractionation. As shown in Figure $6 \mathrm{D}$, immunoblots of subcellular fractions using the N-T TRPV1 antibody (NeuroMab) detected a $90 \mathrm{kD}$ band corresponding to TRPV1 in mouse brain homogenate $(\mathrm{H})$, the nuclear and large membrane pellet fraction $(\mathrm{P})$ indicating that TRPV1 is expressed in the plasma membrane, the synaptosome fraction (P2'), the presynaptic plasma membrane fraction (LP1) and in the "crude" synaptic vesicle fraction (LP2). The N-T antibody did not recognize TRPV1 in the fractions corresponding to the synaptic cytosol (LS2) and "crude" 


\section{RESULTS}

brain cytosol and small organelles (S2). The presynaptic protein synaptobrevin 2 (syb2) as expected was present in all subcellular fractions except the LS2 fraction, and was enriched in the synaptic vesicle fraction.

To further determine if TRPV1 is present in pre- or post-synaptic compartments we conducted a synaptosomal limited proteolysis assay (Figure 6 E). Isolated synaptosomes form in such a way that the presynaptic terminal reseals, enclosing presynaptic components inside, while the post-synaptic side of the synapse remains attached but does not reseal and is therefore accessible the solution. When isolated synaptosomes are subjected to limited proteolysis with trypsin, presynaptic proteins are protected and post-synaptic proteins are digested. The post-synaptic localization of PSD-95, as a positive control, and TRPV1 was confirmed by the detection of a lighter band corresponding to cleavage by trypsin. As shown in Figure $6 \mathrm{E}$ a lower molecular weight band of $\sim 65 \mathrm{kD}$ was recognized by the N-T TRPV1 (NeuroMab) antibody (Table 1) in purified synaptosomes from adult mouse brain homogenates subjected to proteolysis for $10-90$ min, indicating that at least some TRPV1 is embedded in post-synaptic membranes in isolated synaptosomes. Unlike PSD 95, which was largely digested, a full length TRPV1 band at $90 \mathrm{kD}$ remained, meaning that a portion of TRPV1 was not digested and is likely to be presynaptic. Synaptobrevin, a positive control for a presynaptic protein, was not digested at all (Figure $6 \mathrm{E}$ ).

\subsubsection{Expression of functional TRPV1 in hippocampal neuronal cultures}

Since its discovery, TRPV1 is known to be permeable to cations including $\mathrm{Ca}^{2+}$ (Caterina et al., 1997). Monitoring $\mathrm{Ca}^{2+}$ influx upon TRPV1 activation would then allow us to determine if TRPV1 is expressed in hippocampal neurons as active, functional channels. By examining the influx of $\mathrm{Ca}^{2+}$ in neurons upon $1 \mu \mathrm{M}$ capsaicin stimulation we detected approximately $24 \%$ of all hippocampal WT neurons in vitro responded to capsaicin treatment as shown in and quantified in 
Figure $7 \mathrm{~A}-\mathrm{B}$. Only neurons that responded with increases $\mathrm{Ca}^{2+}$ to $40 \mathrm{~Hz}$ field stimulation prior to addition of capsaicin were included in analysis and quantitation. Only $0.69 \%$ of analyzed TRPV1 KO cells showed a slight response to capsaicin. None $(0 \%)$ of the neurons analyzed responded to DMSO used as a positive control.

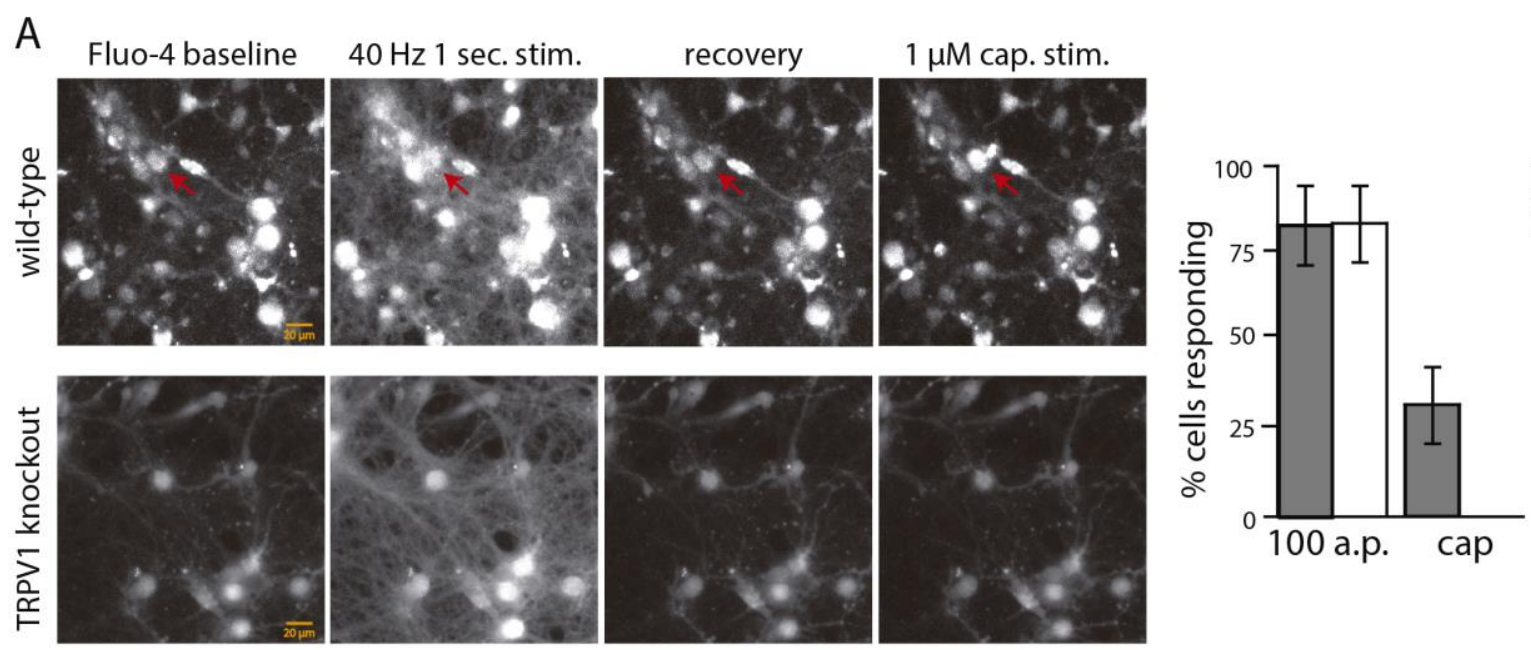

B

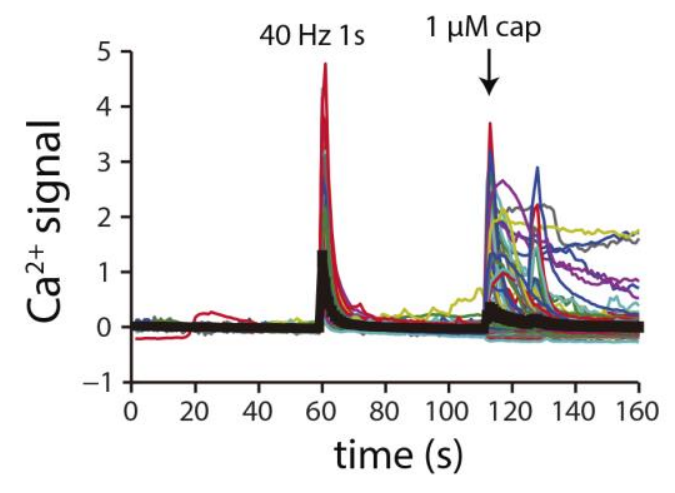

wild-type

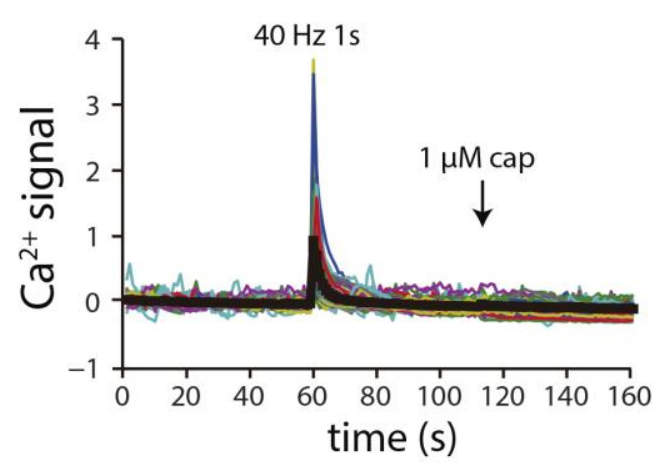

TRPV1 KO

Figure 7. Functional TRPV1 expression in mouse hippocampal neurons in vitro. A) Fluo-4based intracellular $\mathrm{Ca}^{2+}$ imaging. 24\% (B) of hippocampal neurons in WT cultures showed $\mathrm{Ca}^{2+}$ influx upon $1 \mu \mathrm{M}$ capsaicin treatment (red arrow), and TRPV1 $\mathrm{KO}$ cultures showed no $\mathrm{Ca}^{2+}$ influx in response to capsaicin ( $n=19$ images each from 3 different cultures) ( $0 \%$ of neurons reacted to DMSO teatment) (scale bar $=20 \mu \mathrm{m}$ ). B) Representative (colored) and average (black) traces of intracellular Fluo-4 signal show $\mathrm{Ca}^{2+}$ levels rising upon electrical field stimulation $(40 \mathrm{~Hz})$ in both WT and TRPV1 KO neurons. $\mathrm{Ca}^{2+}$ levels increased upon application of $1 \mu \mathrm{M}$ capsaicin in WT but not in TRPV1 KO hippocampal cell cultures ( $n=18$ images each, from 3 different cultures); no intracellular $\mathrm{Ca}^{2+}$ elevation was observed in either WT ( $n=16$ images from 3 different cultures) or in TRPV1 KO ( $n=10$ images from 3 different cultures) cultures treated with DMSO solution. For $\mathbf{B}$ the development of the MATLAB code for analysis was done by Ankit Awasthi. 


\section{RESULTS}

Although the peak of fluorescent signal of $\mathrm{Ca}^{2+}$ influx during electrical stimulation and upon capsacin treatment was variable among different neurons, usually, the capsaicin response was $50 \%$ lower than the response to electrical stimulation. The kinetics of the fluorescence signal for capsaicin-dependent $\mathrm{Ca}^{2+}$ influx was also different than those following $40 \mathrm{~Hz}$ stimulation. The decay was more abrupt after electrical stimulation than for capsaicin where the decay was more gradual and in some cases did not decay completely to baseline before the termination of recording (Figure $7 \mathrm{~A}-\mathrm{C}$ ).

In cultured TRPV1 KO hippocampal neurons we did not detect a significant $\mathrm{Ca}^{2+}$ influx in response to $1 \mu \mathrm{M}$ capsaicin treatment (Figure $7 \mathrm{~A}-\mathrm{C}$ ). Even if a minuscule rise was detected, it was similar to the insignificant increase upon DMSO addition used as a positive control. Intriguingly, the $\mathrm{Ca} 2+$ fluorescence signal detected in response to electrical stimulation was on average two times smaller in TRPV1 KO neurons compared to WT (Figure 7 A-C).

So far, our results confirmed that TRPV1 channels are expressed in the rat and mouse brain during embryonic and postnatal development, continuing throughout adulthood. In the hippocampus, channels are expressed in an active state; possibly in a small subpopulation of neurons. To investigate the specific sub-population of neurons that express TRPV1, we used immunofluorescence techniques in hippocampal neurons in vitro as well as in fully developed hippocampus slices.

\subsection{TRPV1 IS HIGHLY EXPRESSED IN A SPECIFIC HIPPOCAMPAL NEURONAL CELL-TYPE}

Little is known about what neurons express TRPV1 in the hippocampus. It has previously been suggested that only Cajal-Retzius cells express TRPV1 channels in hippocampus. Since Cajal-Retzius neurons eventually die in adulthood, they 
were hypothesized to be short-lived and not to confer a substantial influence in the adult hippocampus (Cavanaugh et al., 2011).

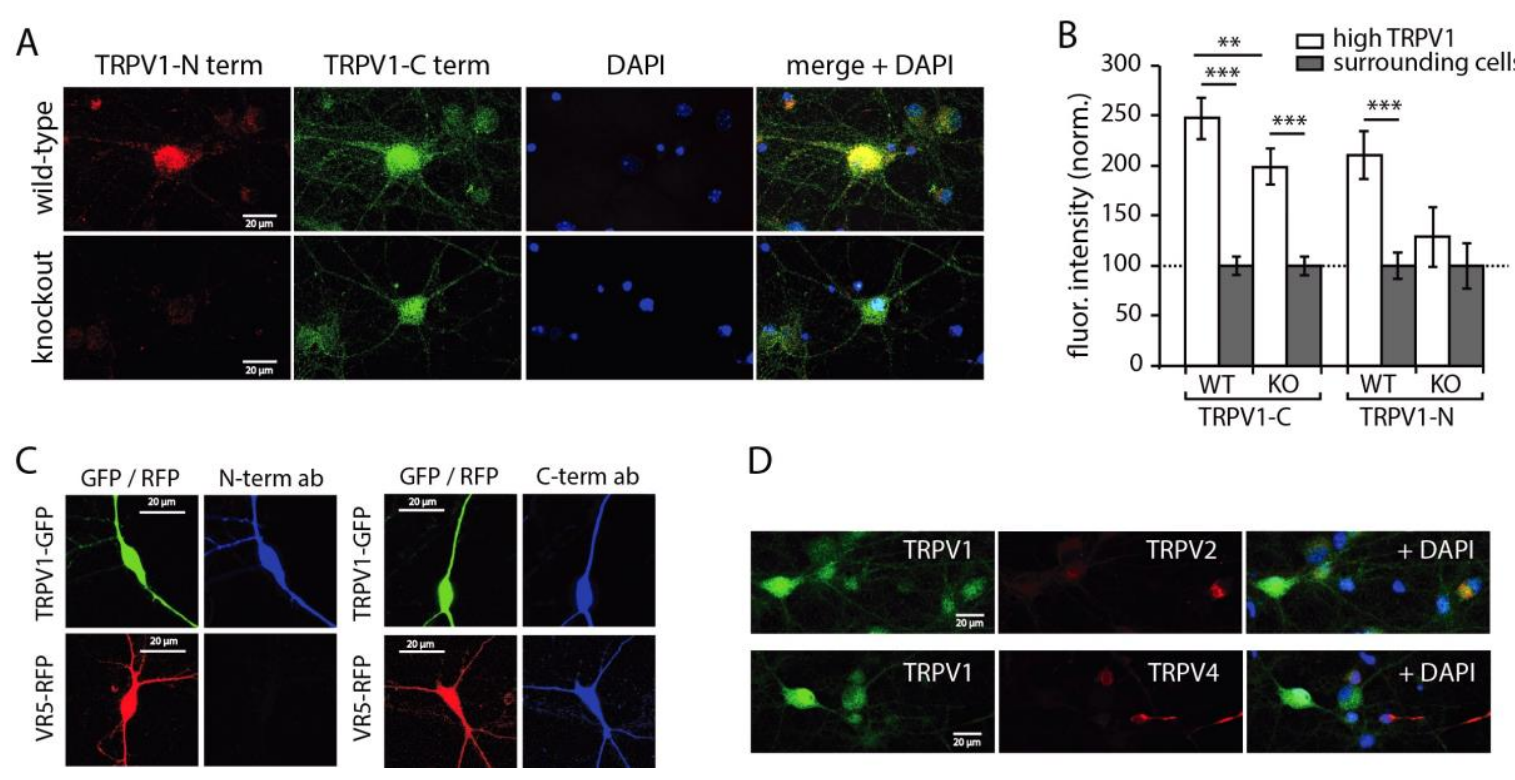

Figure 8. Expression of the TRPV1 N-terminal alternative splice isoform VR.5' sv in the hippocampus. A) Immunocytochemistry of mouse hippocampal neurons in vitro. A subset of hippocampal neurons expresses high levels of TRPV1. Immunofluorescence signal for the N-T TRPV1 antibody (NeuroMab) is absent in TRPV1 KO hippocampal cultures ( $n=6$ of 2 independent neuronal cultures) compared to WT ( $n=6$ of 2 independent neuronal cultures). Immunofluorescence signal of the C-T TRPV1 antibody (Millipore) observed in WT neurons $(n=12$ of 4 different neuronal cultures) remains in TRPV1 KOs ( $n=12$ of 4 different neuronal cultures) as quantified in (B) (error $=$ SEM, significance determined by unpaired Student's t-test ${ }^{* *} p<0.01,{ }^{* * *} p<0.001$ ) (scale bar $=20$ $\mu \mathrm{m})$. B) Fluorescent intensity of high-expressing neurons normalized to that of surrounding neurons of a given immunostaining. C) Both the C-T and the N-T TRPV1 antibodies detect mouse hippocampal neurons transfected with the TRPV1-EGFP construct. The C-T TRPV1 antibody, but not the N-T antibody detects neurons transfected with the VR.5' sv-RFP construct (scale bar $=20$ $\mu \mathrm{m})$. D) The C-T TRPV1 antibody does not recognize the highly homologous TRPV2 or TRPV4 channels, which are highly expressed in a different subset of mouse hippocampal neurons in vitro ( $n=6$ for each staining of 2 independent neuronal cultures) (scale bar $=20 \mu \mathrm{m}$ ).

When we conducted immunostainings in WT mouse hippocampal cell cultures using the N-T TRPV1 antibody, we found that only a small number of neurons were highly expressing TRPV1, which had on average twice as much fluorescence intensity as surrounding neurons (Figure $8 \mathrm{~A}$ and $\mathrm{B}$ ). We were not able to find any TRPV1-positive cells using this antibody in TRPV1KO cultures. However, we found that these same neurons were still detected by the C-T antibody in TRPV1 KO hippocampal cultures, which had twice the amount of fluorescence intensity as surrounding neurons (Figure $8 \mathrm{~A}$ and $\mathrm{B}$ ). Co-stainings for the $\mathrm{C}$ vs $\mathrm{N}$ terminus 


\section{RESULTS}

antibodies showed that in these cells, the signal of the N-T antibody observed in WT conditions was gone in TRPV1 KO cultures (Figure $8 \mathrm{~A}$ and $\mathrm{B}$ ). Interestingly, the $C$-T-specific immunofluorescence in these neurons was significantly decreased in TRPV1KO compared to WT cultures.

\subsubsection{High TRPV1 expressing neurons in the stratum oriens}

Similar to ICC, IHC of adult mouse hippocampus using the TRPV1 C-T antibody showed that only a small subpopulation of neurons express high levels of TRPV1, and such neurons were mainly present in the stratum oriens (Figure $9 \mathrm{~A}$ and $\mathrm{B}$ ). Nevertheless, TRPV1-positive neurons are not just restricted to SO. Some of them are also present in the CA1, CA2 and CA3 regions. The minority of TRPV1-positive neurons were observed in the pyramidal layer and polymorph layer of the dentate gyrus.

The localization of high TRPV1-expressing neurons in the SO cells recalled that of somatostatin-expressing OLM (oriens lacunosum moleculare) neurons, which are long known to be present in that region of the SO (Sik et al., 1995). We found that most high TRPV1-expressing neurons also express somatostatin in the SO (Figure $9 \mathrm{~B})$.

Similar to the observations made in vitro, some neurons in the hippocampus were still recognized by the C-T specific TRPV1 antibody in slices of TRPV1 KOs, including somatostatin-positive neurons of the SO (Figure $9 \mathrm{C}$ ). Nevertheless, the number of detected neurons in whole hippocampus was about $40 \%$ less in TRPV1 $\mathrm{KO}$ compared to WT mice, as quantified in Figure $9 \mathrm{C}-\mathrm{E}$. Interestingly, when we used the N-T antibody (Neuromics) a sharper drop in the number of neurons with high TRPV1 expression was observed in KO hippocampus compared to WT. The number of neurons with high TRPV1 expression was reduced more than $80 \%$ (Figure $9 \mathrm{E}$ ). 


\section{RESULTS}

A
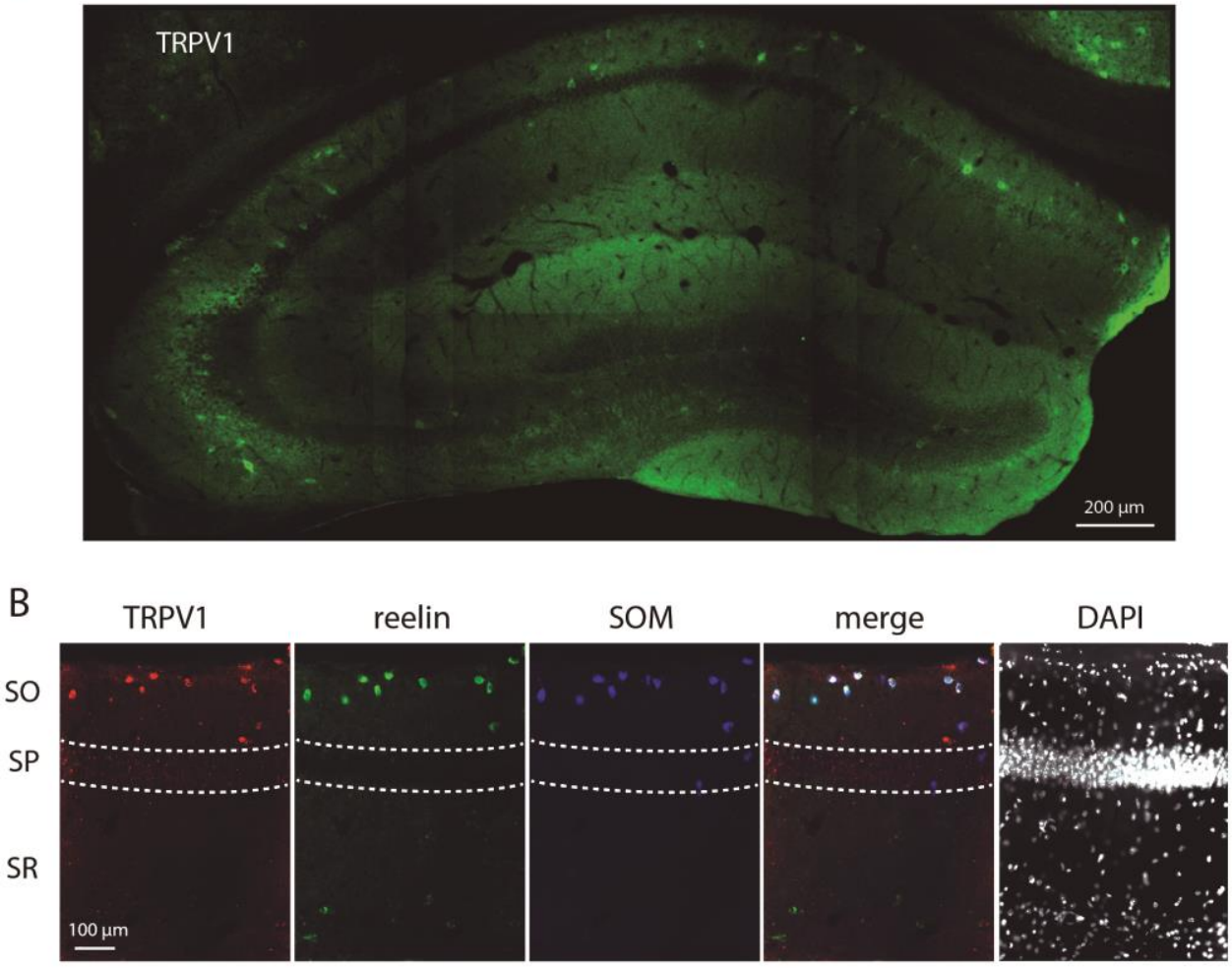

C

wild-type
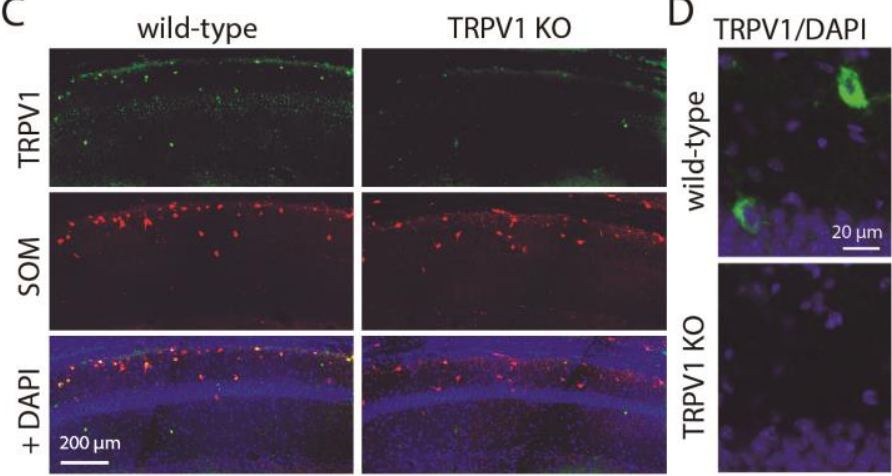

$\mathrm{E}$

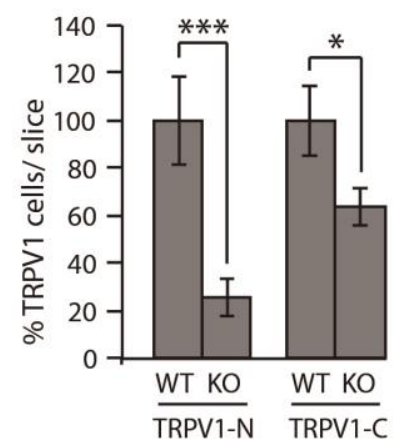

Figure 9 TRPV1 is highly expressed in OLM neurons in the hippocampus. A) Immunohistochemistry in slices of the mouse hippocampus reveals a subset of neurons with high expression of TRPV1, mainly in the stratum oriens (scale bar $=100 \mu \mathrm{m}$ ). B) As showed in vitro, somatostatin and reelin (markers of OLM interneurons) co-localize with high TRPV1 expressing neurons in the stratum oriens (SO: stratum oriens, SP: stratum pyramidale, SR: stratum radiatum) (scale bar $=100 \mu \mathrm{m}$ ). C) Representative area of the CA1 region of a WT and TRPV1 KO hippocampus immunostained with the TRPV1 C-T antibody (Millipore), somatostatin and DAPI (scale bar $=100 \mu \mathrm{m}$ ). D) Zoom of a high TRPV1 expressing neuron in the stratum oriens in WT and the corresponding region in TRPV1 KO hippocampal slices (scale bar $=20 \mu \mathrm{m}$ ). E) Quantitation of TRPV1 N-T and C-T antibody signal in WT and TRPV1 KO hippocampal slices Immunofluorescence of N-T TRPV1 antibody is almost absent in the hippocampus of TRPV1 KO animals. In some neurons in TRPV1 KO slices low signal of the C-T antibody remains (possibly corresponding to a TRPV1 splice isoform). But the number TRPV1 C-T positive cells is reduced in the hippocampus of TRPV1 KOs (Percentages of TRPVI KO normalized to the corresponding WT values; $n=5$ slices each for WT and TRPV1 KO). For $\mathbf{B}, \mathbf{C}$ and $\mathbf{D}$ technical assistance was provided by Christiane Bolleyer and Patricia Sprysch. 


\section{RESULTS}

First, we thought that possibly the C-T antibody recognized a different TRPV channel highly homologous to the TRPV1 channel, but as shown in Figure $8 \mathrm{D}$ we found TRPV2 and TRPV4 expressed in different neurons, or at least in cells not recognized by the C-T antibody. It is known that other splice isoforms are expressed in the hippocampus (e.g. VR.5' sv), which could also be recognized by TRPV1 antibodies. As shown in section 4.1.1, the VR.5' sv (which has the same CT sequence as TRPV1) mRNA is present in WT hippocampus and is significantly increased in TRPV1 $\mathrm{KO}$ animals. As shown in Figure $8 \mathrm{C}$, the $\mathrm{C}-\mathrm{T}$ antibody (Millipore) recognized both TRPV1- and VR.5' sV-transfected hippocampal neurons in vitro, yet the $\mathrm{N}-\mathrm{T}$ antibody (Neuromab) only recognized neurons transfected with TRPV1. These results suggest that TRPV1 antibodies at least partially recognize other TRPV1 isoforms that could have different physiological properties.

\subsubsection{TRPV1 expression in oriens lacunosum moleculare inhibitory interneurons}

As suggested in the section above, high TRPV1 expression occurs in a specific neuronal cell-type with delimited localization in the adult hippocampus. In the stratum oriens these neurons express somatostatin (a marker for OLM neurons).

We confirmed the inhibitory nature of high TRPV1-expressing neurons by verifying their expression of glutamate decarboxylase 65 (GAD 65), an enzyme involved in GABA synthesis (Erlander and Tobin, 1991). As shown in Figure 4 A, ICC of rat hippocampal neurons in vitro indicates that the vast majority of the total counted neurons that express high levels of TRPV1 co-localized with GAD-65, as quantified in Figure $10 \mathrm{~B}$. According to a previous study using a TRPV1 reporter mouse (Cavanaugh et al., 2011) TRPV1 should be expressed in reelin-positive neurons, specifically, in Cajal-Retzius cells, that are known to express reelin (Del Río et al., 1997). As shown in Figure 10 A and B (in vitro) and Figure 9 B (slices) we corroborated that TRPV1 is highly expressed in reelin-containing neurons. In vitro, approximately $90 \%$ of the TRPV1-positive neurons co-localized with reelin and half 
of the reelin-positive neurons were not positive for TRPV1 (Figure $10 \mathrm{~B}$ ). Of total TRPV1 neurons, 62\% colocalized with somatostain (Figure $10 \mathrm{~B}$ ). Interestingly, none of the high TRPV1-expressing neurons expressed VIP or Parvalbumin (markers for other inhibitory neurons) (Figure $10 \mathrm{~B}$ ).

A
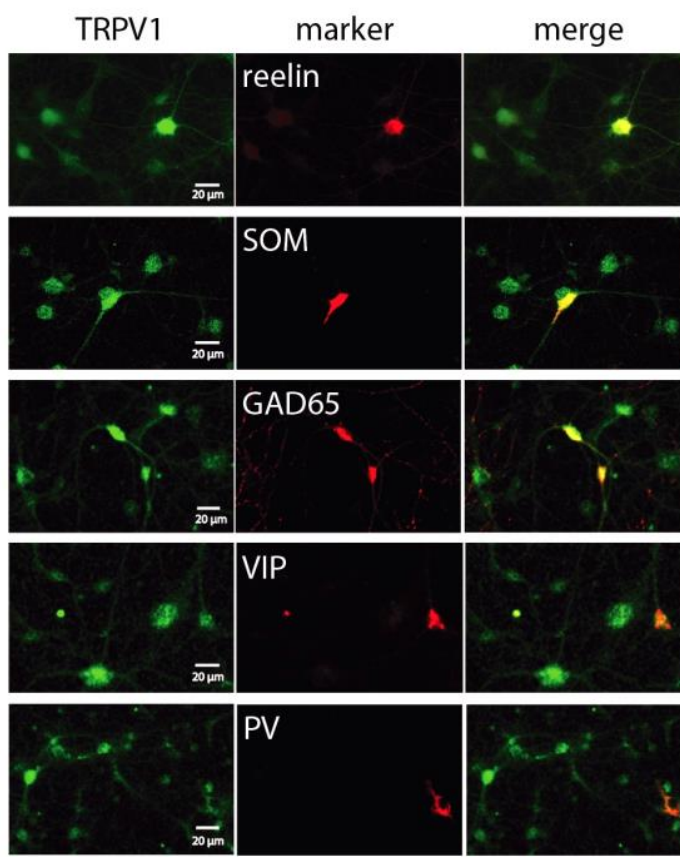
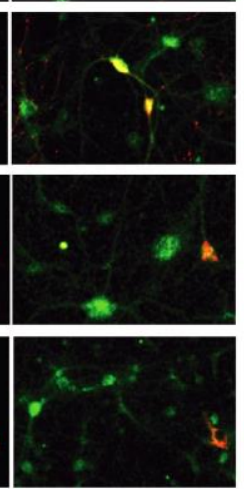

B
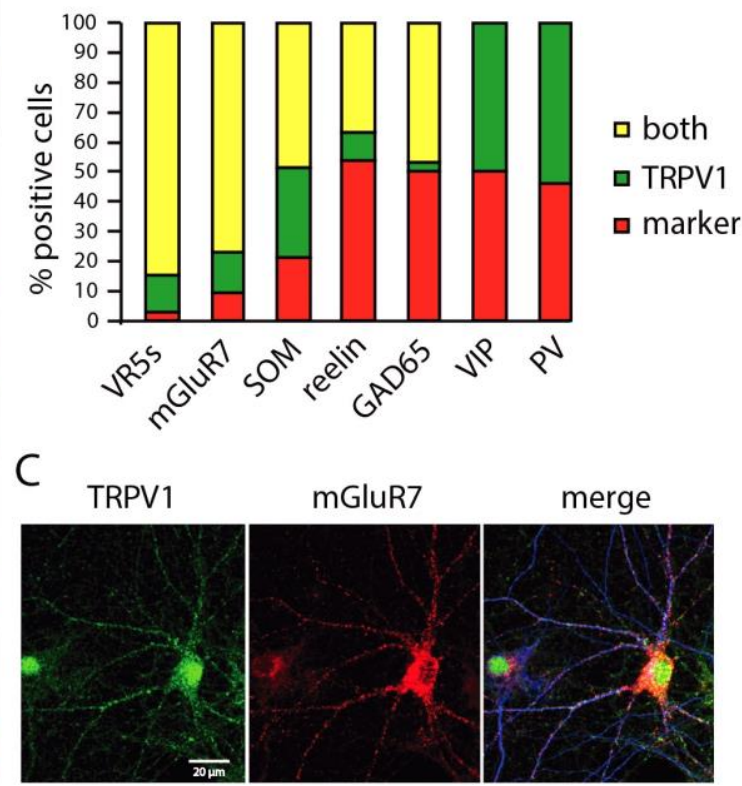

Figure 10 High TRPV1 expression in a subset of hippocampal inhibitory interneurons in vitro. A) Immunocytochemical analysis in rat hippocampal cultures at DIV 12 identifies a subpopulation of inhibitory interneurons (GAD65 positive) with elevated TRPV1 channel expression which co-express somatostatin and reelin (markers for OLM neurons) but not parvalbumin or VIP. B) Quantification of co-localization of immunofluorescence signal of different markers with TRPV1 neurons in rat hippocampal cell cultures at DIV12; data normalized to the total number of neurons counted (considered as 100) for each analysis. C) Inhibitory interneurons expressing high levels of TRPV1 have an increased number mGluR7-positive puncta (cells analyzed: TRPV1/NR.5' sv $n=54$, TRPV1/somatostatin $n=62$, TRPV1/reelin $n=52$, TRPV1/VIP $n=22$, TRPV1/PV $n=26$, TRPV1/GAD65 $n=60$, TRPV1/mGluR7 $n=22$; obtained from 3-6 independent neuronal cultures). Scale bar $=20 \mu \mathrm{m}$.

Because reelin is an important signaling molecule necessary for the proper formation of cortical and hippocampal laminae during both pre- and post-natal development (Caviness and Rakic, 1978; Stranahan et al., 2013) and since we have found a substantial co-localization of TRPV1 in reelin-expressing neurons we inquire if TRPV1 KO animal would have an altered hippocampal and brain barrel architecture. Nonetheless we fail to detect any obvious structural alteration in the conformation of the brain (Figure $11 \mathrm{~A}$ ). 


\section{RESULTS}

We found that neurons having increased immunofluorescent signal using the C-T antibody in vitro not only expressed reelin but they also were positive for somatostatin (Figure 15). In hippocampal slices TRPV1 positive neurons of the SO were also expressing somatostatin and reelin (Figure 9 B) indicating that these kind of neurons are not Cajal-Retzius cells but they belong to a different population of inhibitory interneurons; colocalization of these two markers has been previously reported in SO in OLM neurons (Alcántara et al., 1998; Chittajallu et al., 2013). Thus, the TRPV1 expressing neurons in SO are most likely OLM interneurons and not Cajal-Retzius neurons.

To further test the idea that TRPV1 is expressed in higher levels in OLM neurons we performed ICC against the metabotropic glutamate receptor 7 (mGluR7) which has been reported to be present in presynaptic terminals innervating OLM neurons (Shigemoto et al., 1996, 1997; Tomioka et al., 2014). We found that $80 \%$ of TRPV1-expressing neurons in vitro had high levels of mGluR7 in terminals contacting them, whereas only $10 \%$ of neurons expressed exclusively either TRPV1 or mGluR7 (Figure $10 \mathrm{~B}$ and $\mathrm{C}$ ). 


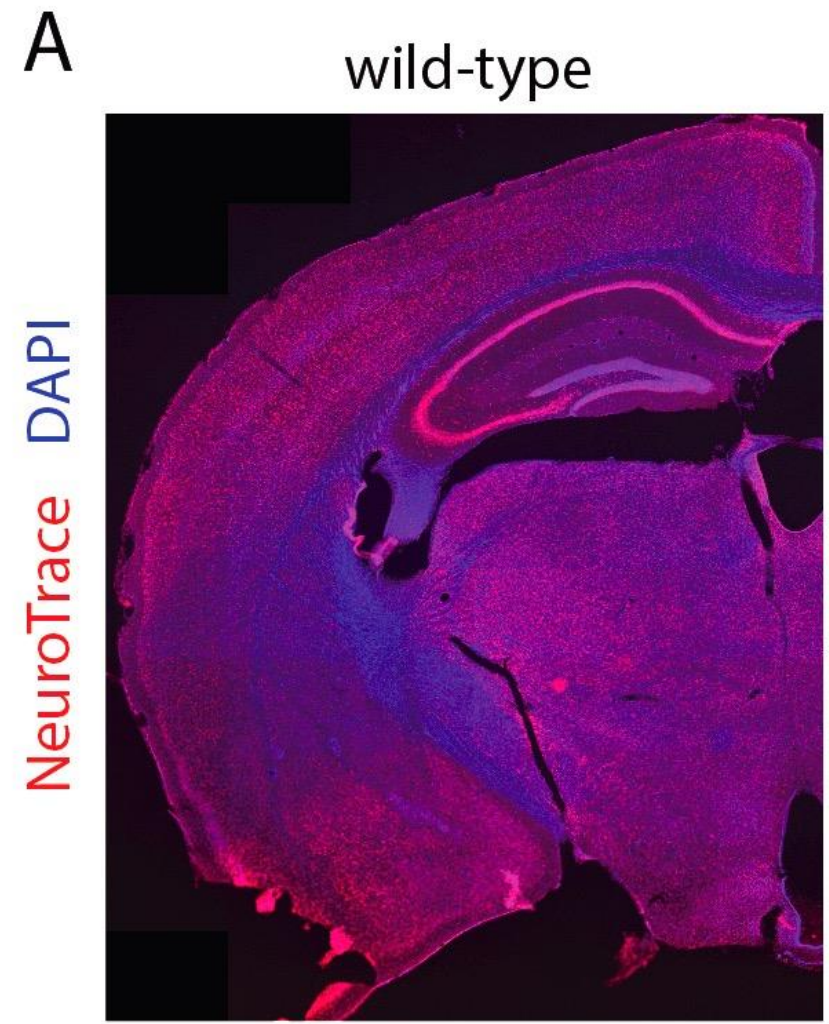

\section{TRPV1 KO}

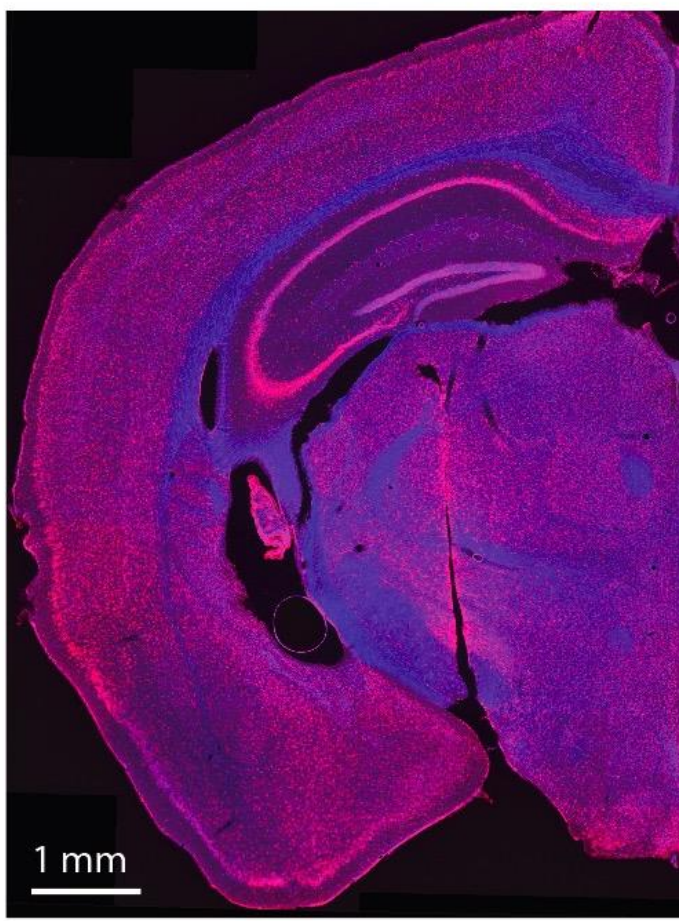

Figure 11 TRPV1 deficient mice have a normal cortical and hippocampal laminar

architecture. A) There was no obvious alteration in gross morphology of the adult brain in TRPV1 KOs compared to WT, assayed by Neurotrace and DAPI staining (scale bar $=1 \mathrm{~mm}$ ). Technical assistance provided by Robin Wagener and Patricia Sprysch.

\subsection{TRPV1 EXPRESSION IN OLM NEURONS MODULATES EXCITATORY SYNAPTIC STRENGTH}

OLM neurons are known to modulate excitatory synaptic transmission through GABAergic inhibition of distal dendrite tufts of pyramidal neurons at the Schaffer collateral pathway of the CA1 region (Klausberger et al., 2004). It has been just recently found that OLM neurons mostly receive excitatory inputs from pyramidal neurons of the CA1 region of the hippocampus (Sun et al., 2014). Less is known about the inhibitory input to OLM neurons. It has been suggested that OLM neurons receive GABAergic innervation from bistratified or trilaminar interneurons (Leão et al., 2012), interneuron-specific type III expressing VIP neurons (Acsády et 


\section{RESULTS}

al., 1996; Blasco-lbáñez and Freund, 1995) and even from other OLM neurons populating the CA1 region of the hippocampus (Kogo et al., 2004).

\subsubsection{TRPV1 channel activity influences excitatory pre- synaptic strength in OLM neurons}

We found that OLM neurons having high TRPV1 expression are highly innervated by excitatory synaptic boutons both in vivo (Figure $12 \mathrm{~A}$ ) and in vitro (Figure $12 \mathrm{~B}$ ) compared to surrounding neurons. OLM neurons growing in vitro also have more GABA-positive synaptic terminals contacting them compared to other neurons, although this difference is not as extreme the difference in glutamatergic terminals.

Is TRPV1 linked to the augmented excitatory and inhibitory innervation of OLM neurons? First we evaluated if in vitro, weak and strong pharmacological activation of TRPV1 using either $50 \mathrm{nM}$ or $1 \mu \mathrm{M}$ capsaicin incubation (ca. 21-24 h) would have any influence on presynaptic strength. Immunocytochemically we inspected characteristics of excitatory and inhibitory presynaptic strength by monitoring the amount of vGluT1 (pre-synaptic excitatory marker) and vGAT (pre-synaptic inhibitory marker) puncta number as well as the fluorescent intensity of both markers together with the analysis of concomitant signal for Syt1u - an antibody against the lumenal domain of synaptotagmin1, which is taken up by recycling synaptic vesicles during depolarization and acts as a readout for presynaptic strength. 

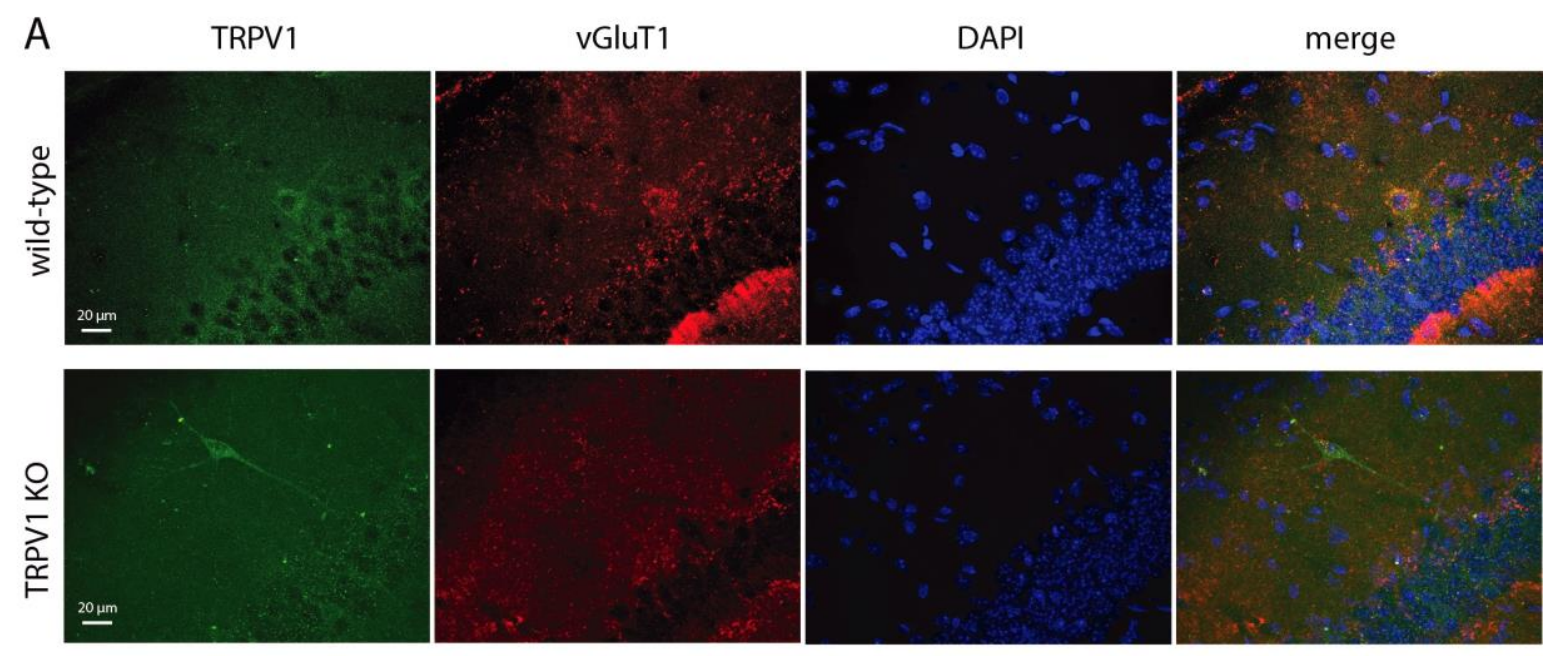

B
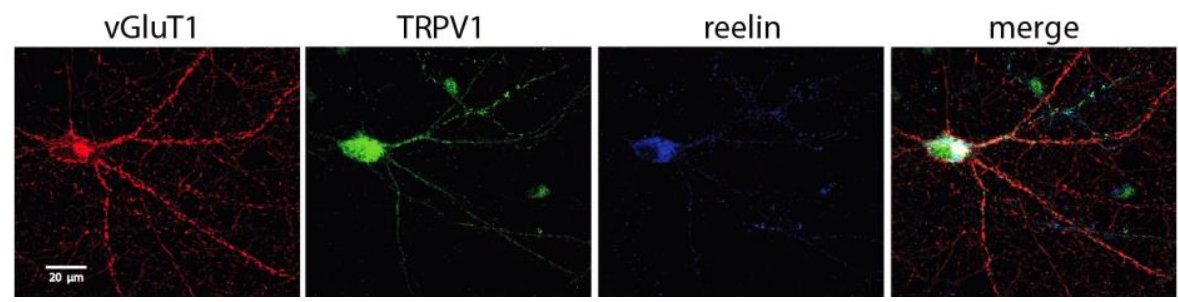

Figure 12. Increased excitatory innervation of high TRPV1 expressing OLM neurons. A) Representative image of IHC of hippocampal slices of WT and TRPV1 KO mice showing the stratum oriens (scale bar $=50 \mu \mathrm{m}$ ). B) High-expressing TRPV1 neurons in hippocampal cell cultures have an elevated number of vGluT1 positive puncta, which is reduced in TRPV1 KOs (scale bar $=20 \mu \mathrm{m})$.

Analysis of the fluorescence intensity and number of presynaptic marker puncta (corresponding to synapses) showed that in control conditions high TRPV1 expressing neurons had at least twice as the vGluT1 (a marker for excitatory synaptic boutons) intensity and puncta number corresponding to synapes, compared to surrounding low-expressing TRPV1 neurons (Figure $13 \mathrm{~A}-\mathrm{E}$ ). The number of vGluT1-stained excitatory synapses increased upon $50 \mathrm{nM}$ capsaicin treatment, and increased even more with a higher concentration of $1 \mu \mathrm{M}$ capsaicin stimulation, while vGluT1 fluorescence intensity in both high and low-expressing TRPV1 cells did not change upon capsaicin treatment. Interestingly, $1 \mu \mathrm{M}$ capsaicin also doubled the number of vGluT1 puncta on surrounding neurons compared to untreated conditions. However syt1u fluorescence intensity decreased in both high and low TRPV1-expressing neurons treated with $1 \mu \mathrm{M}$ capsaicin. When we applied a TRPV1 antagonist, SB-366791, together with $1 \mu \mathrm{M}$ 
capsaicin, the observed effect of capsaicin treatment was reverted. On average, treatment with $1 \mu \mathrm{M}$ SB antagonist reduced both the number and intensity of vGluT1 immunostained puncta (Figure $13 \mathrm{~A}-\mathrm{E}$ ).

A

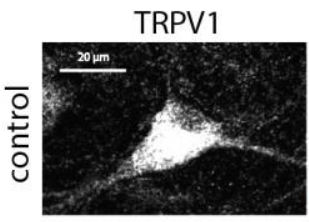

B

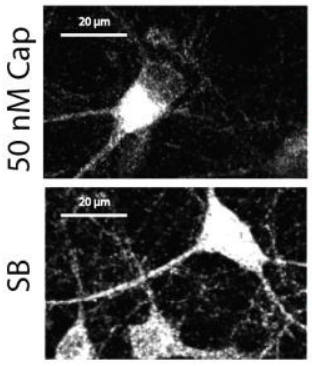

control
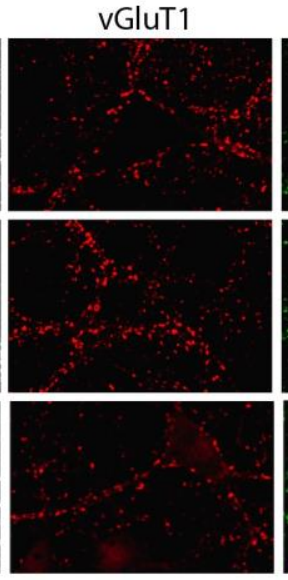

50 nM Cap
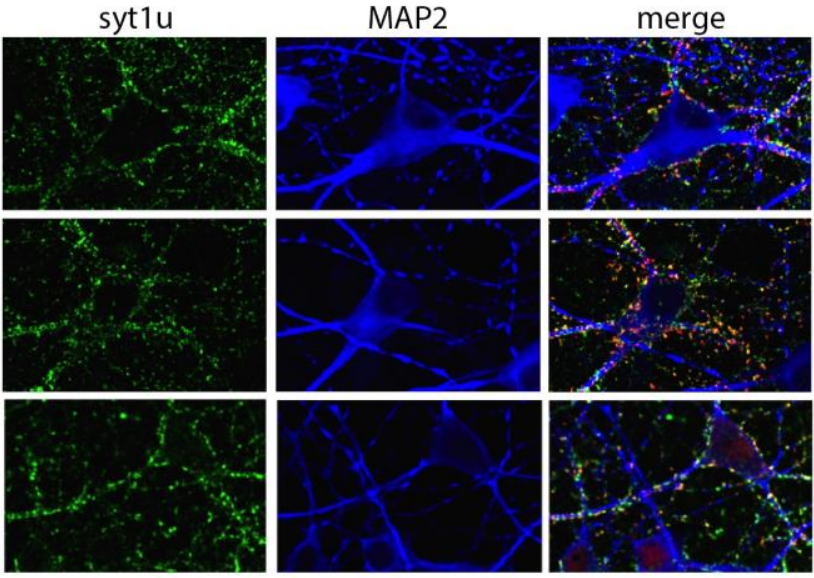

SB

$1 \mu \mathrm{M}$ Cap

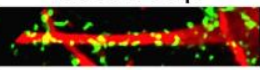

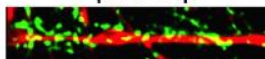

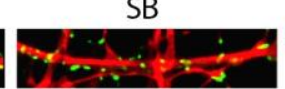

TRPV1 KO

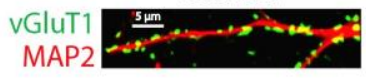

C

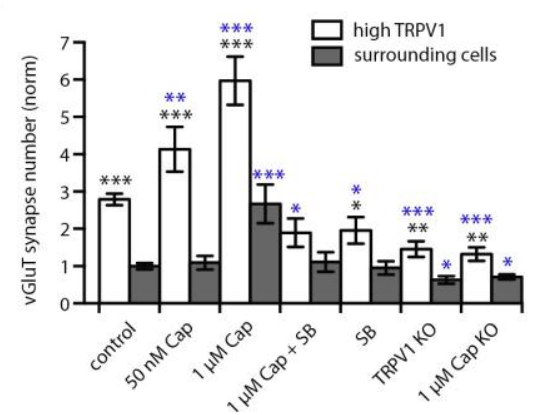

D

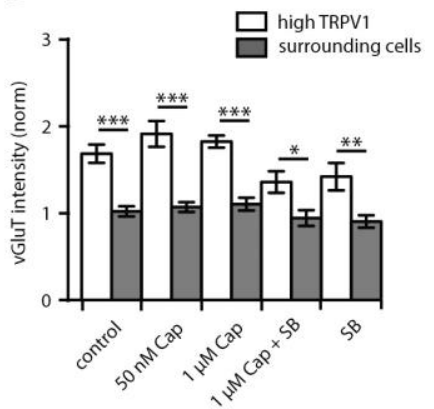

E

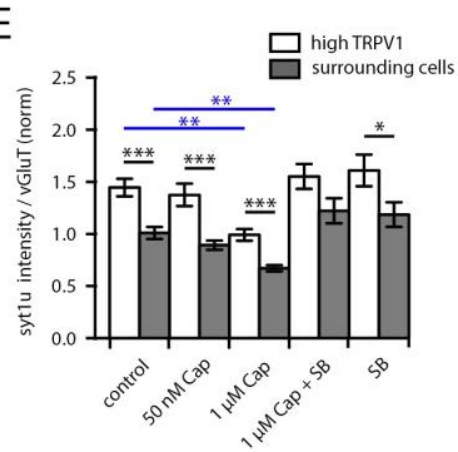

Figure 13 Post-synaptic TRPV1 promotes excitatory innervation of OLM cells. A) Quadruple ICC of rat neurons immunostained for TRPV1, vGluT1, syt1 internalized by recycling synaptic vesicles during depolarization, and MAP2 in rat hippocampal cultures in the indicated conditions (scale bar $=20 \mu \mathrm{m}$ ). B) ICC of vGluT1 and MAP2 in mouse hippocampal cultures SB-366791 (scale bar $=5 \mu \mathrm{m}$ ). C) Quantitation of excitatory synapse number (number of vGluT1-positive puncta) on high-expressing TRPV1 hippocampal neurons compared to surrounding cells in the indicated conditions. D) Quantitation of vGluT1 intensity (total excitatory synaptic vesicle number) in presynaptic terminals contacting high-expressing TRPV1 or surrounding neurons of rat hippocampal cell cultures, in the indicated conditions. E) Quantitation of syt1 uptake in depolarizing conditions (total number of recycling excitatory synaptic vesicles) in vGluT1-positive terminals contacting highexpressing TRPV1 or surrounding neurons, in the indicated conditions (Images: WT Control $n=24$, WT cap. 50nM $n=12$, WT cap. $1 \mu \mathrm{M} n=9$, WT cap. $1 \mu \mathrm{M}+\mathrm{SB} 1 \mu \mathrm{M} n=12$, WT SB $1 \mu \mathrm{M} n=12$, TRPV1 KO control $n=9$, TRPV1 KO cap $1 \mu \mathrm{M} n=9$; from 3-8 different neuronal preparations; error bar $=$ SEM; significance determined by unpaired Student's t-test ${ }^{*} p<0.05,{ }^{* \star} p<0.01,{ }^{* \star *} p<0.001$; black asterisks: significant difference between high-expressing and surrounding neurons in each condition, blue asterisks: significant difference between conditions for high-expressing TRPV1 cells, or between conditions for surrounding cells; fluorescent intensity and synapse number for all conditions was normalized to the corresponding average value for surrounding neurons in WT cell cultures). 
A
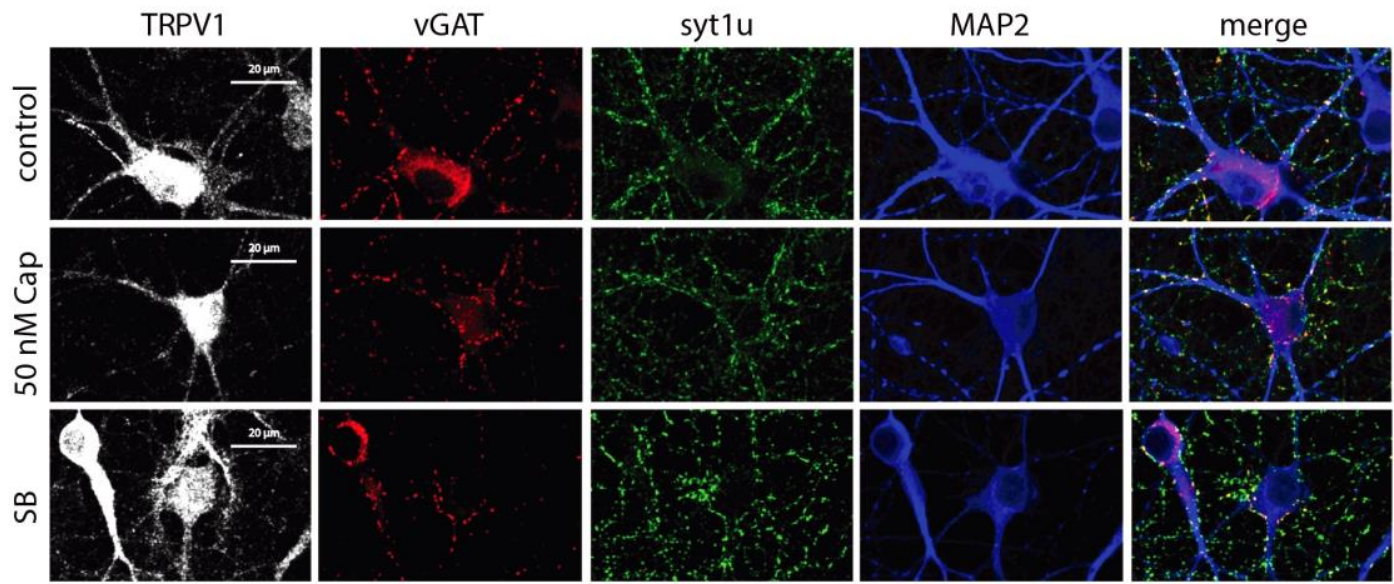

B
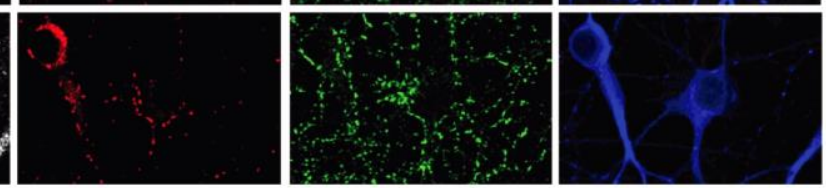

1 MM Cap

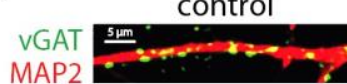

50 nM Cap

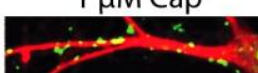

SB

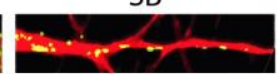

TRPV1 KO

C

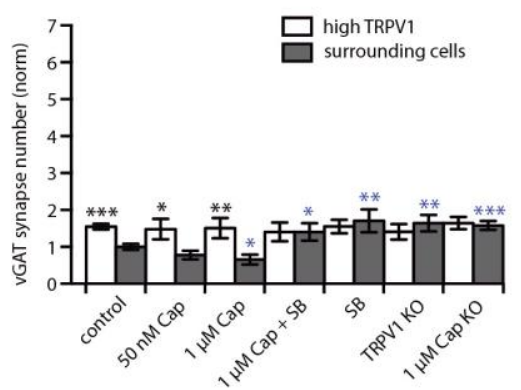

D

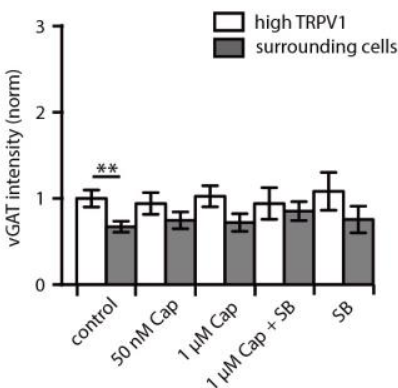

E

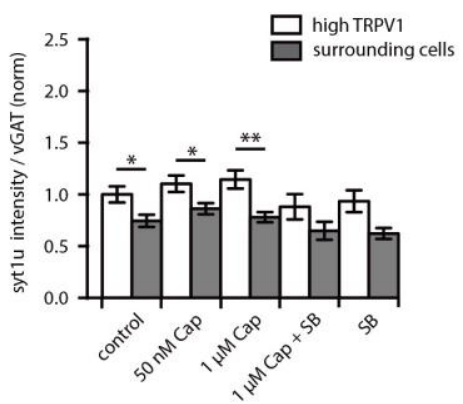

Figure 14 Inhibitory innervation of OLM neurons is less affected by post-synaptic TRPV1 expression. A) Quadruple ICC of rat neurons immunostained for TRPV1, vGAT, syt1 internalized by recycling synaptic vesicles during depolarization, and MAP2 in rat hippocampal cultures in the indicated conditions (scale bars $=20 \mu \mathrm{m}$ ). B) ICC of vGluT1 and MAP2 in mouse hippocampal cultures C) Quantitation of excitatory synapse number (number of vGAT-positive puncta) on highexpressing TRPV1 hippocampal neurons compared to surrounding cells in the indicated conditions. D) Quantitation of vGAT intensity (total excitatory synaptic vesicle number) in presynaptic terminals contacting high-expressing TRPV1 or surrounding neurons of rat hippocampal cell cultures, in the indicated conditions (scale bars $=5 \mu \mathrm{m}$ ). E) Quantitation of syt1 uptake in depolarizing conditions (total number of recycling excitatory synaptic vesicles) in vGAT-positive terminals contacting highexpressing TRPV1 or surrounding neurons, in the indicated conditions (Images: WT Control $n=23$, WT cap. 50nM $n=11$, WT cap. $1 \mu \mathrm{M} n=9$, WT cap. $1 \mu \mathrm{M}+$ SB $1 \mu \mathrm{M} n=12$, WT SB $1 \mu \mathrm{M} n=12$, TRPV1 KO control $n=9$, TRPV1 KO cap $1 \mu M n=9$, images obtained from 3-8 independent neuronal cell cultures; error bar $=\mathrm{SEM}$; significance determined by unpaired Student's $t$-test * $p<0.05$, ** $\mathrm{p}<0.01,{ }^{* * *} \mathrm{p}<0.001$; black asterisks: significant difference between high-expressing and surrounding neurons in each condition, blue asterisks: significant difference between conditions for high-expressing TRPV1 cells, or between conditions for surrounding; fluorescent intensity and synapse number for all conditions was normalized to the corresponding average value for surrounding neurons in WT cell cultures).

In control, $50 \mathrm{nM}$ and $1 \mu \mathrm{M}$ capsaicin conditions, high TRPV1-expressing neurons also had more inhibitory synapses than other cells. Unexpectedly, we saw that 1 $\mu \mathrm{M}$ capsaicin treatment mildly reduced the number of vGAT stained puncta in 


\section{RESULTS}

neurons with lower TRPV1 immunofluorescence (Figure 14 A-E). The SB-366791 TRPV1 channel blocker reverted the effects of capsaicin by increasing the number of vGAT puncta to control levels. Intriguingly, when SB-366791 was applied alone, it also increased the number of VGAT-positive puncta only on low TRPV1expressing neurons (Figure $14 \mathrm{~A}-\mathrm{E}$ ).

\subsubsection{TRPV1 expression increases excitatory but not inhibitory synapse innervation in OLM neurons}

Since we detected that modifying activity of TRPV1 expressed in OLM neurons can regulate the formation of excitatory synapses onto OLM neurons we used the TRPV1 KO model to confirm the synaptogenic effects of TRPV1 in this subclass of interneurons. OLM neurons in TRPV1 KO cultures were identified using somatostatin and reelin as markers. Only neurons colocalizing with both markers were selected for analysis. Independent measurements in TRPV1 KO vs WT mouse hippocampal neurons were also conducted using the TRPV1 C-T antibody signal as a marker (which remained in TRPV1 KO animals) (see section 4.3.1).

OLM neurons had on average twice as many vGluT1 synapses as surrounding neurons in WT conditions (Figure 15 A-B) Impressively, we found that the number of excitatory terminals forming onto OLM neurons was drastically reduced (by 3 times) in TRPV1 KO cultures compared to WT. The decrease was so pronounced that we could not find a significant difference in the number of excitatory synapses on OLM neurons vs non-OLM neurons (Figure $15 \mathrm{~A}-\mathrm{B}$ ). As shown for rat cultures (Figure $13 \mathrm{~B}-\mathrm{E}$ ), $1 \mu \mathrm{M}$ capsaicin doubled the number of glutamatergic inputs onto OLM neurons in WT hippocampal cultures (Figure $15 \mathrm{~A}-\mathrm{B}$ ). The effects of $1 \mu \mathrm{M}$ capsaicin on excitatory synapse number were absent in TRPV1 KO OLM neurons (identified with somatostatin and reelin) (Figure $15 \mathrm{~A}$ and B). In general, the same results were observed when using the $\mathrm{C}-\mathrm{T}$ antibody signal as a marker for selection (instead of somatostatin/reelin) of putative OLM neurons (Figure $13 \mathrm{~B}$ and $\mathrm{C})$. 
A
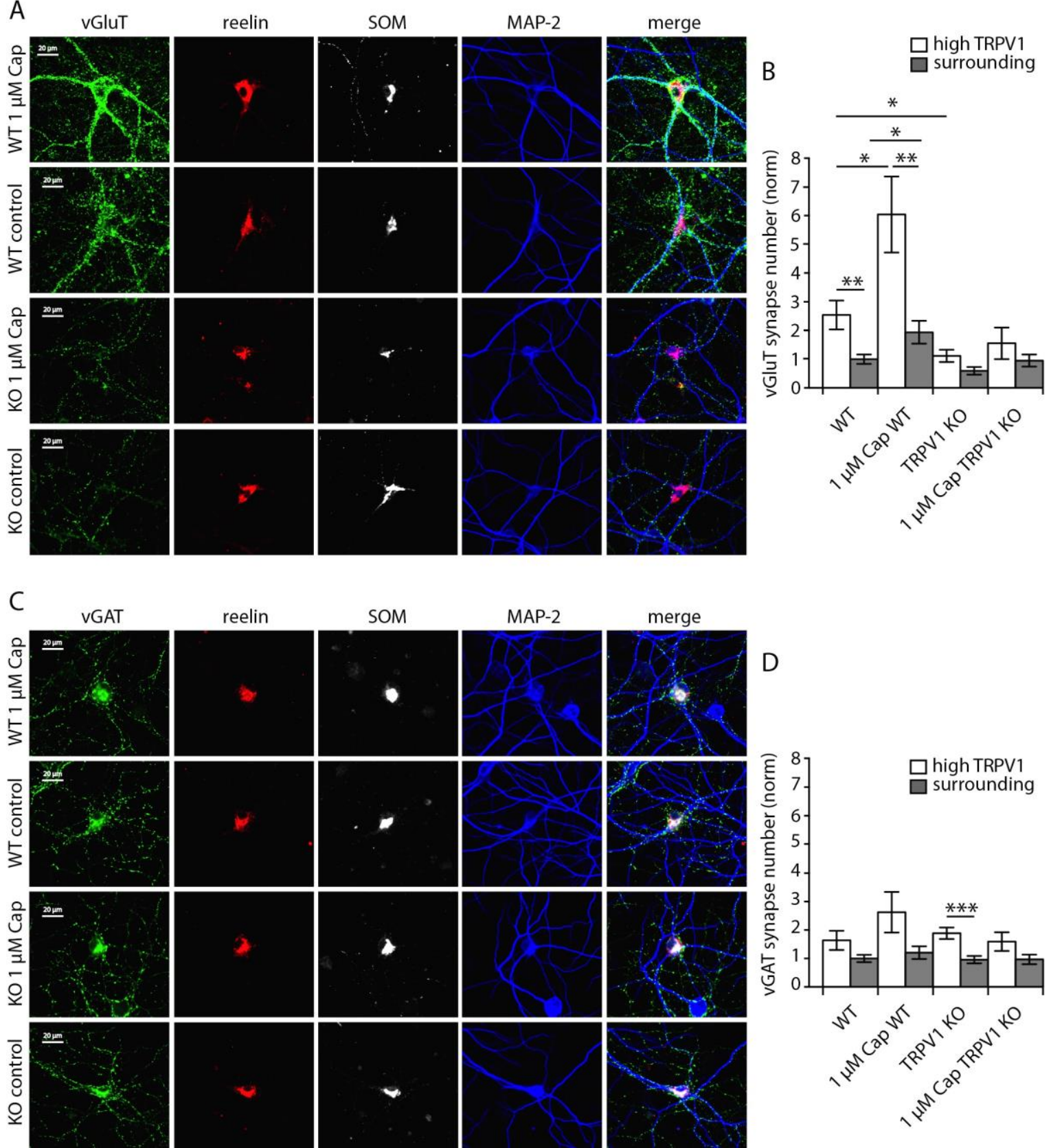

Figure 15. TRPV1 promotes excitatory innervation of OLM neurons. A) Quadruple ICC of DIV12-13 WT and TRPV1 KO hippocampal neurons shows that OLM neurons co-expressing somatostatin and reelin have increased excitatory innervation (vGluT1 signal) compared to surrounding cells (quantified in B). Neuronal processes are shown by MAP2 staining. TRPV1 KO neurons have a dramatic reduction in excitatory innervation, compared to WT. $1 \mu \mathrm{M}$ capsaicin treatment increased the number of vGluT1-positive synapse in WT neurons ( $n=20$ images for all conditions) C) Quadruple ICC of mouse hippocampal cell cultures for vGAT, to mark inhibitory synapses, reelin, somatostatin, and MAP2. No difference was observed in the number of inhibitory synapses in WT and TRPVI KO neuronal cultures. Inhibitory synapse number is not influenced by 1 $\mu \mathrm{M}$ capsaicin treatment, quantified in (D) Although OLM neurons tend to have slightly more vGATpositive synapse, the difference is minor. ( $n=20$ images for all conditions of 2 independent neuronal cell culture dissection; error bar $=\mathrm{SEM}$, statistical significance determined by Student's unpaired ttest, ${ }^{*} p<0.05,{ }^{* *} p<0.01,{ }^{* * *} p<0.001$; values fluorescent intensity for all conditions normalized to the average intensity of surrounding non-OLM neurons of WT cell cultures). 


\section{RESULTS}

We found that TRPV1 acts as a synaptogenic regulator of excitatory synapses, but TRPV1 activation, blockade or removal did not significantly affect the amount of inhibitory synapses on OLM interneurons. OLM neurons in TRPV1 KO cultures had the same amount of VGAT-stained synapses as WT neurons, and the number of VGAT-positive synapses in these neurons was not altered by $1 \mu \mathrm{M}$ capsaicin treatment (Figure $15 \mathrm{~B}-\mathrm{C}$ ).

These results reflect previously unknown synaptogenic properties of TRPV1 in OLM neurons, which may affect synaptic plasticity coordinated by inhibitory interneurons in the SO.

\subsection{HETEROLOGOUS TRPV1 OVEREXPRESSION INCREASES EXCITATORY INNERVATION}

To determine if TRPV1 can promote excitatory synapse formation in cells that don't normally express it at high levels, DIV2 hippocampal neurons were transfected with TRPV1 expressed under the $\beta$-actin promoter to express TRPV1 during synaptogenesis in vitro and validate if overexpression of TRPV1 would affect synapse formation. It is important to note that TRPV1 is expressed at E18 when embryonic rat hippocampal preparation was conducted (Figure $6 \mathrm{C}$ ). Neurons were fixed at DIV 12-13, that is $10-11$ days after transfection.

\subsubsection{TRPV1 overexpression at DIV2 in hippocampal neurons increases excitatory innervation}

Low efficiency transfection of TRPV1 channel at DIV2 and overexpression for 10 days caused an increase in the number of glutamatergic terminals contacting transfected neurons. Not only the number of excitatory terminals was increased, but also the fluorescence intensity of vGluT1 and the co-localizing syt1u 


\section{RESULTS}

immunofluorescence signal (Figure 16 A-D). We corroborated this effect by measuring mEPSC amplitude and frequency in TRPV1-transfected neurons, where the frequency (thought to correspond to an increase in synapse number or synaptic vesicle release probability) and not the amplitude was higher than in EGFP transfected neurons (Figure $16 \mathrm{E}$ ). Blockade of the channel at DIV4 reverted the effects of TRPV1 overexpression on glutamatergic pre-synaptic strength (i.e. number of synapses, vGluT1 and syt1u fluorescence intensity) to levels similar to EGFP transfected neurons (Figure 16 A-D).

When TRPV1 and VR.5' sv were co-transfected at DIV2 this also caused an increase in the number (and syt1u intensity) of vGluT1-positive synapses contacting the transfected neuron (Figure $16 \mathrm{~F}-\mathrm{I})$. The increase in excitatory synapse number induced by TRPV1/VR.5' sv co-transfection was similar to that observed when TRPV1 was transfected alone. The number and strength of excitatory synapses terminating on neurons transfected with VR.5' sv only were not significantly changed compared to control transfections (Figure 16 F-I).

Unexpectedly, heterologous expression of TRPV1 in cultured hippocampal neurons before synapse formation also had significant effect on GABAergic presynaptic strength by decreasing both the average fluorescent intensity of VGAT immunostained synapses and of the corresponding syt1u signal (Figure $17 \mathrm{~A}-\mathrm{C}$ ). The number of inhibitory inputs formed on the transfected neurons remained unchanged (Figure $17 \mathrm{D}$ ). The frequency and amplitude of mIPSCs in TRPV1 transfected neurons were similar to EGFP-transfected control neurons (Figure 17 E). Treatment of TRPV1 transfected neurons with SB at DIV4 decreased the average intensity of VGAT-positive puncta and reverted the effects observed in the syt1u fluorescent intensity caused by the overexpression with TRPV1 (Figure 17 B and C). 


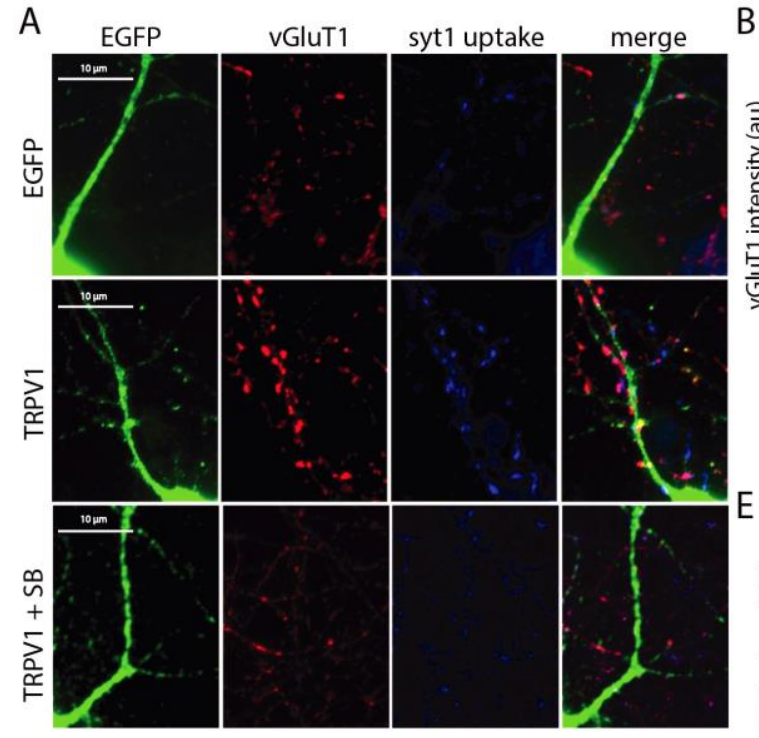

B

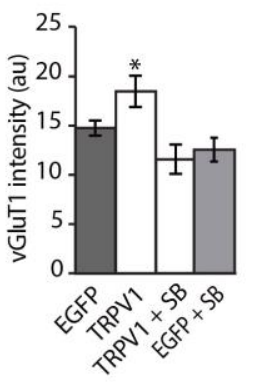

C

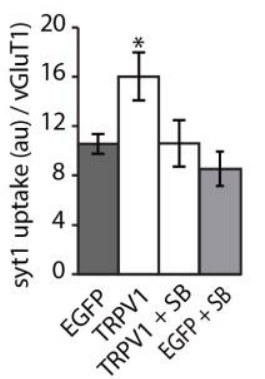

D
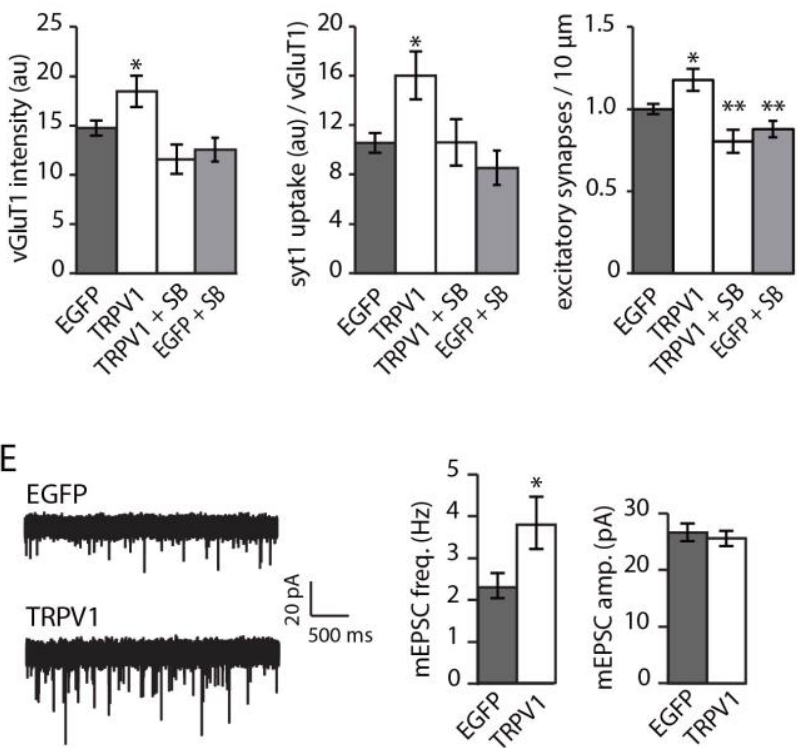

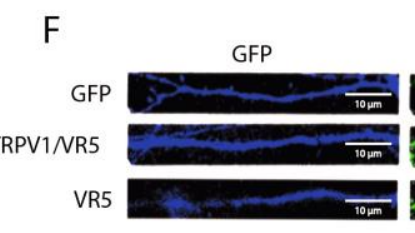

G

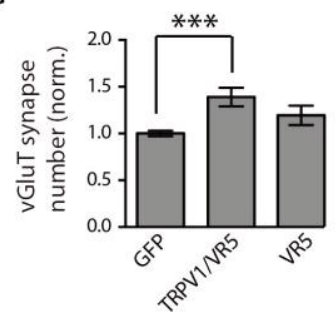

VGluT

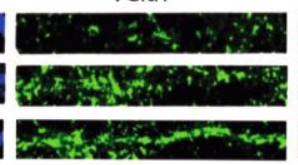

$\mathrm{H}$

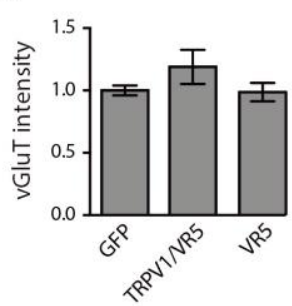

syt1 uptake

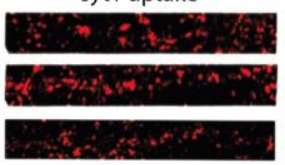

merge

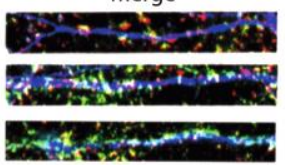

I

Figure 16. TRPV1 and TRPV1/VR.5' sv overexpression in neurons increases excitatory innervation. A) Triple ICC for vGluT1 and syt1u of rat hippocampal neurons transfected with TRPV1-IRES-GFP or EGFP before synapse formation on DIV2 (scale bars $=10 \mu \mathrm{m}$ ). B) Quantitation of the excitatory synaptic strength, assayed by vGluT1 signal and overlapping syt1u fluorescence intensity (C) D) Quantitation of excitatory synapse number (number of vGluT1 puncta) on TRPV1-expressing cells with and without treatment with SB-366791 compared to EGFP (Images: EGFP; $n=60$, TRPV1; $n=30$, TRPV1 + SB-366791; $n=15$; images obtained from 5-20 different neuronal cultures; all values normalized to the average value obtained for EGFP transfected neurons). E) TRPV1-overexpressing neurons have increased mEPSC frequency compared to control EGFP-expressing neurons, and amplitude remained unchanged, consistent with an increase in synapse number in TRPV1-overexpressing neurons (cells: EGFP $n=29$, TRPV1 DIV2 $n=27)$. F) Triple ICC for vGluT1 and syt1u of rat hippocampal neurons transfected with TRPV1/VR.5' sv, VR.5' sv or EGFP before synapse formation on DIV2 (svale bars $=10 \mu \mathrm{m}$ ) G) Quantitation of excitatory synapse number, excitatory synaptic strength, assayed by vGluT1 signal (H) and overlapping syt1u fluorescence intensity (I) (Images: EGFP $\mathrm{n}=15-21$, TRPV1 DIV2 $\mathrm{n}=11$ 15, TRPV1/VR.5' sv DIV2 n=12-15, VR.5' sv DIV2 n=10-13; images obtained from 4-7 different cell culture preparation; error $=\mathrm{SEM}$; significance determined by Student's unpaired t-test ${ }^{*} p<0.05$, ${ }^{* *}$ $p<0.01,{ }^{* * *} p<0.001$; all values normalized to the average value obtained for EGFP transfected neurons). Immunostainings for $\mathbf{F}$ and analysis for $\mathbf{G}, \mathbf{H}$ and $\mathbf{I}$ conducted in collaboration with Christiane Bolleyer. 


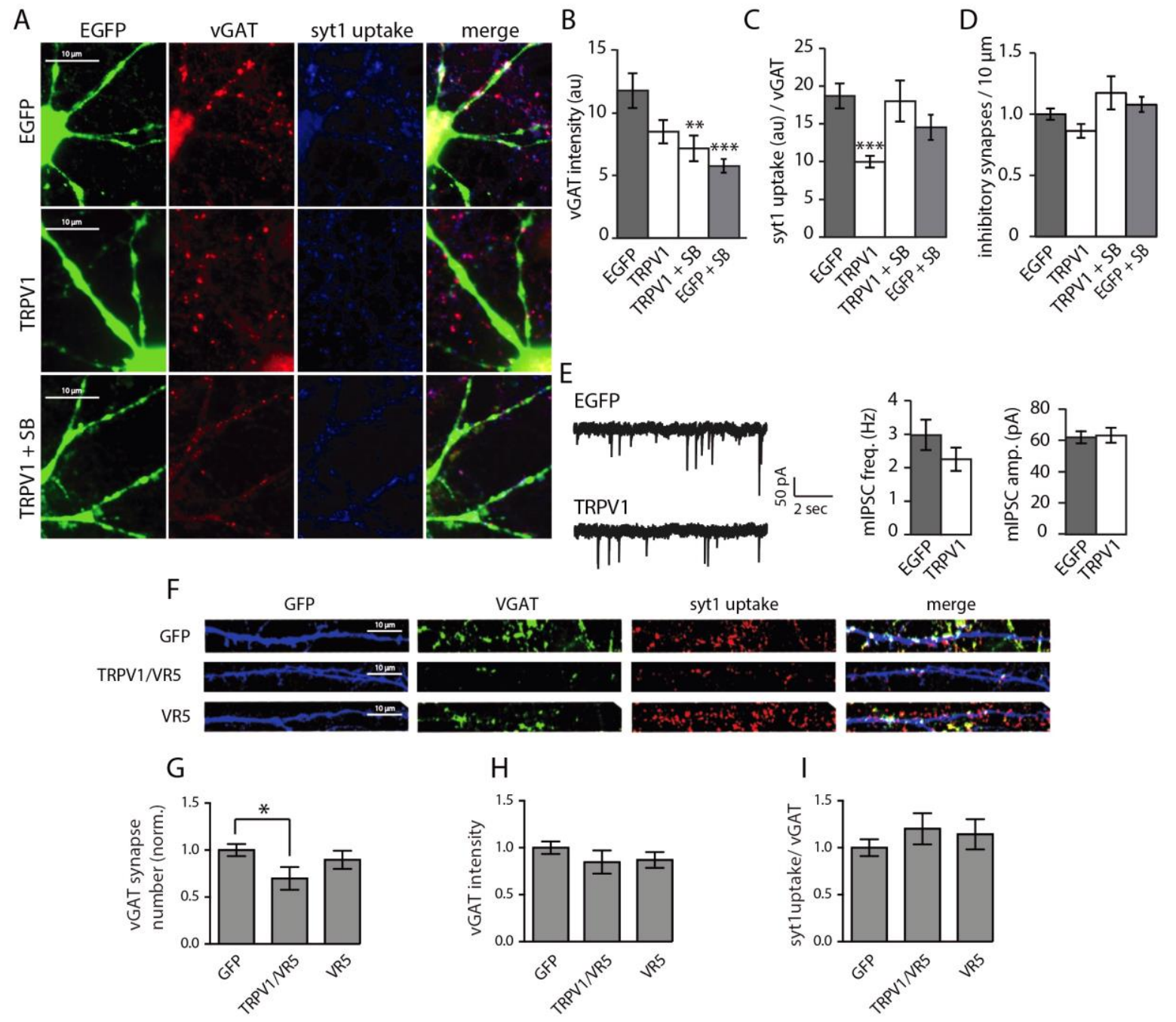

Figure 17. TRPV1/VR.5' sv overexpression in neurons decreases inhibitory innervation. A) ICC for vGAT and syt1u of rat hippocampal neurons transfected with TRPV1-IRES-GFP or EGFP before synapse formation on DIV2 (scale bars $=10 \mu \mathrm{m}$ ). B) Quantitation of the excitatory synaptic strength, assayed by vGluT1 signal and overlapping syt1u fluorescence intensity (C) D) Quantitation of inhibitory synapse number (number of vGAT puncta) on TRPV1-expressing cells with and without treatment with SB-366791 compared to EGFP (Images: EGFP n=60, TRPV1 DIV2 $\mathrm{n}=27$, TRPV1 DIV2 + SB-366791 $1 \mu$ M DIV4 $n=15$; images taken from 5-20 different neuronal cell cultures; synapse number for all conditions was normalized to the corresponding average value for surrounding neurons in WT cell cultures). E) TRPV1-overexpressing neurons have no change in mIPSC frequency or amplitude, compared to control EGFP-expressing neurons (cells: EGFP $n=27$, TRPV1 DIV2 $n=24)$. F) Triple ICC for vGAT and syt1u of hippocampal neurons transfected with TRPV1/VR.5' sv, VR.5' sv and EGFP before synapse formation on DIV2 (scale bars $=10 \mu \mathrm{m}$ ). G) Quantitation of inhibitory synapse number, excitatory synaptic strength, assayed by vGAT signal (H) and overlapping syt1u fluorescence intensity (I) (Images: EGFP $n=12-15$, TRPV1 DIV2 $n=12-15$, TRPV1/VR.5' sv DIV2 $n=9-12$, VR.5' sv DIV2 $n=9-11$; images taken from 3-5 independent cell cultures; error = SEM; significance determined by unpaired Student's t-test ${ }^{*} p<0.05,{ }^{* *} p<0.01,{ }^{* * *}$ $\mathrm{p}<0.001$; synapse number for all conditions was normalized to the corresponding average value for surrounding neurons in WT cell cultures). Immunostainings for $\mathbf{F}$ and analysis for $\mathbf{G}, \mathbf{H}$ and $\mathbf{I}$ conducted in collaboration with Christiane Bolleyer. 


\section{RESULTS}

When TRPV1 and VR.5' sv were co-transfected at DIV2 this caused a decrease in the number vGAT synapses contacting transfected neurons (Figure $17 \mathrm{~F}$ and $\mathrm{G}$ ). The number and strength of inhibitory synapses terminating on neurons transfected with VR.5' sv only were not significantly changed compared to control transfections (Figure 17 F-I).

\subsection{TRPV1 EFFECTS ON SCHAFFER COLLATERAL LTP AND LTD}

\subsubsection{TRPV1 does not affect LTD in the Schaffer collateral pathway of the CA1 region of hippocampus}

Previous studies have shown that TRPV1 activation is involved in changes in LTD induction in the nucleus accumbens (Grueter et al., 2010), dentate gyrus (Chávez et al., 2010) and in LTD modulation at excitatory synapses onto CA1 interneurons in the hippocampus (Gibson et al., 2008), which is absent in TRPV1 KO animals (Brown et al., 2013). In field recordings in the Schaffer collateral pathway we did not find any change in the induction of LTD in TRPV1 KO animals compared to hippocampal slices of WT mice (Figure 19 A).

\subsubsection{TRPV1-induced innervation of OLM neurons is necessary for gating of CA3 hippocampal information}

Nicotine is known to gate and promote the induction of LTP in the Schaffer collateral pathway through activation of the highly specific nicotinic acetylcholine receptor a2 subunit (nAChr2) (Fuji et al., 1999; Nakauchi et al., 2007a). Just recently, it has been reported that only OLM neurons express such receptor subunits, and that nicotine-dependent LTP induction in the Schaffer collateral 


\section{RESULTS}

pathway is mediated through an OLM-dependent disinhibitory mechanism (Leão et al., 2012).

A

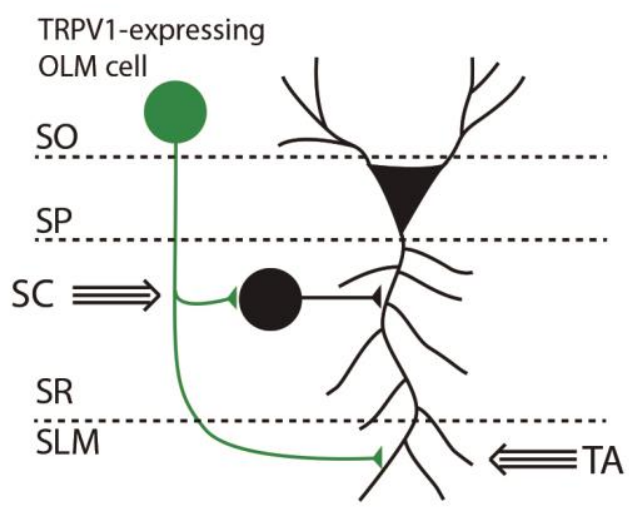

B

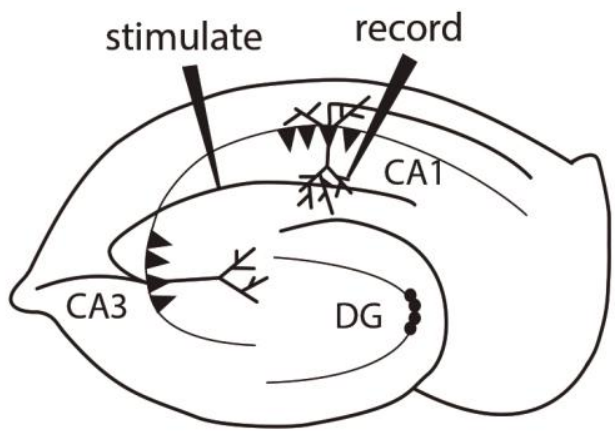

Figure 18. OLM neurons gate synaptic plasticity in the Schaffer collateral. A) Scheme of putative connections of a TRPV1-expressing OLM cell in the stratum oriens. OLM cells inhibit the temporoammonic (TA) pathway from the entorhinal cortex, and dis-inhibit the Schaffer collateral pathway from the CA3 region, thus promoting CA3 input, and reducing EC input, to CA1 pyramidal cells. If excitatory innervation in TRPV1-lacking OLM cells is reduced, inhibition of the TA pathway would be weakened, and dis-inhibition of the Schaffer collateral pathway would be weakend, i.e. TRPV1 KOs would increase inhibition in the Schaffer collateral pathway resulting in decreased Schaffer collateral LTP. B) Illustration of stimulating and recording electrode positions for LTP and LTD recordings performed in the Schaffer collateral pathway.

We tested if TRPV1 would have an effect in the LTP mechanism of the Schaffer collateral pathway by inducing LTP using a 1XTET LTP induction in acute WT and TRPV1 KO hippocampal slices.

Interestingly, a 1 TET protocol resulted in a significant decrease in LTP in the Schaffer collateral pathway in recordings from TRPV1 KO compared to WT (Figure 19 B). This suggested that TRPV1 plays a role in normal hippocampal functioning and plasticity.

TRPV1-KOs have a dramatic reduction in excitatory innervation of OLM neurons, which we hypothesized would result in decreased disinhibition of the SC pathway and the reduced LTP that we, and others (Marsch et al., 2007), have observed. If this is the case, we hypothesized that activation of TRPV1 channels in OLM cells with nicotine would bypass the innervation defects in OLM cells in TRPV1 KOs and 


\section{RESULTS}

rescue the LTP deficits. Indeed, when we treated TRPV1 KO slices with $5 \mu \mathrm{M}$ nicotine prior and during the recordings we found a rescue of LTP to levels similar to WT slices treated with and without nicotine (Figure $19 \mathrm{C}$ ).

A

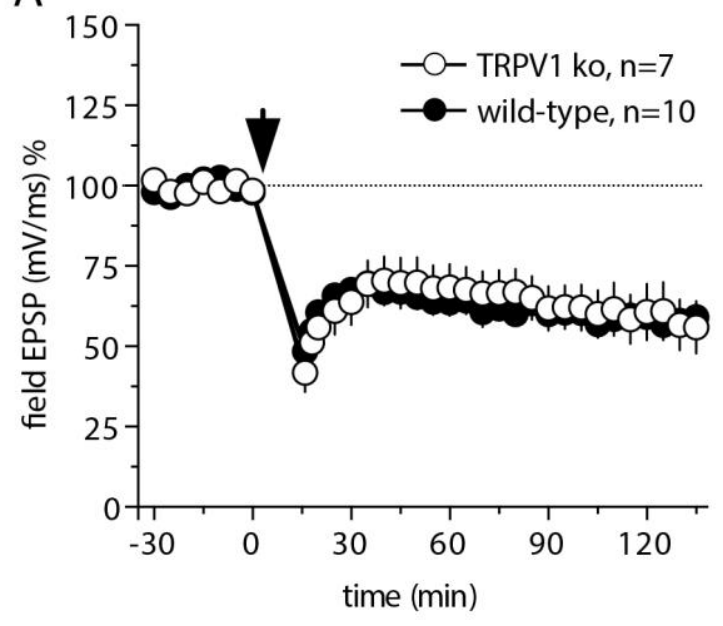

B

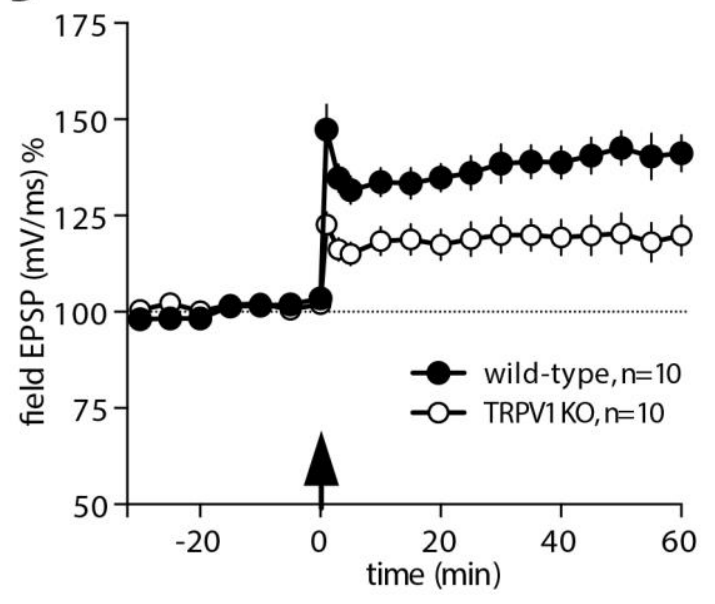

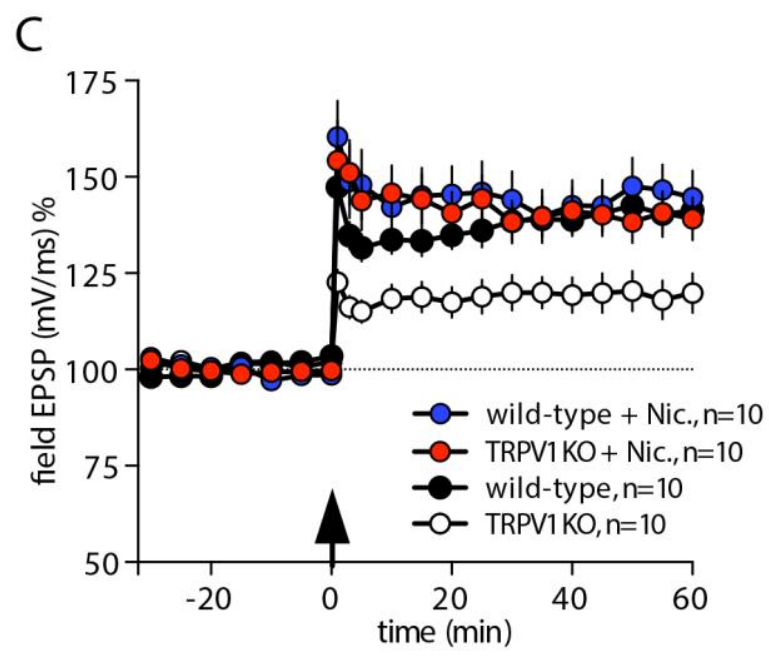

Figure 19. TRPV1 KOs show reduced Schaffer collateral LTP that is rescued by nicotine treatment. A) LTD induced by low-frequency stimulation of the Schaffer collateral pathway is unchanged in TRPV1 KOs. B) LTP induced by 1XTET-LTP in the Schaffer collateral is reduced in TRPV1 KOs compared WT. C) As in (B), LTP in the Schaffer collateral pathway is significantly reduced in TRPV1 KOs and is restored to WT levels by $5 \mu \mathrm{M}$ nicotine treatment 20 minutes before and during recording. LTD and LTP recordings perfomed by Binu Ramachandran. 


\section{RESULTS}

\subsection{REGULATION OF TRPV1 EXPRESSION IN HIPPOCAMPAL NEURONS IN VITRO}

In the PNS, TRPV1 plays a pivotal role in the perception of noxious stimuli such as pain, heat, acidity and pungent agents such as capsaicin (Caterina et al., 1997, 2000). Diverse exogenous and endogenous factors have been reported to activate, sensitize, desensitize, block and regulate expression of TRPV1, especially during nociception. For instance upregulation of TRPV1 expression has been proposed to occur during nerve injury (Hudson et al., 2001), hyperalgesia (Davis et al., 2000; Miranda et al., 2007) and inflammatory pain (Ji et al., 2002; Luo et al., 2004; Schicho et al., 2004; Schwartz et al., 2013). In the model of inflammatory pain, NGF has arisen as an important mediator (Ji et al., 2002) that upregulates TRPV1 expression (Anand et al., 2006; Winston et al., 2001) and trans-location into the plasma membrane (Zhang et al., 2005) of afferent neurons. In the CNS, particularly in the hippocampus just a few studies have pointed out that TRPV1 expression is increased in patients with mesial temporal lobe epilepsy (Sun et al., 2012) and after seizures in rat models for temporal lobe epilepsy (Bhaskaran and Smith, 2010). A more recent study found that mRNA levels of TRPV1 (together with receptors of the endocannabinoid system) were increased in brain and hippocampus of adult rats subjected to stress in early life (Marco et al., 2014). 
RESULTS

\subsection{NGF UPREGULATES TRPV1 EXPRESSION IN HIPPOCAMPAL NEURONS}

\subsubsection{NGF increases TRPV1 expression in hippocampal neurons in vitro}

As mentioned above, NGF is known to increase TRPV1 expression in afferent neurons (i.e. from the dorsal root ganglia). We were interested to compare if TRPV1 expression in young (DIV2-5) and mature (DIV12-13) hippocampal neurons could be modified by NGF. We incubated hippocampal neurons with $1 \mathrm{ng} / \mathrm{ml}$ NGF for 21-24 $\mathrm{h}$ and then measured TRPV1 expression using ICC and WB.

As shown in Figure $20 \mathrm{C}$ and D NGF treatment of cultured rat hippocampal neurons caused an increase in the fluorescence intensity of C-T TRPV1 antibody signal in cultures both before and after the main period of synaptogenesis. The increase in TRPV1 expression was more pronounced in mature (DIV12-13) than in young (DIV2-5) cell cultures. To further verify the NGF-induced increase in TRPV1 expression we quantified protein expression signal detected by the N-T TRPV1 antibody by WB. The changes we observed by ICC were corroborated with WB for NGF treatment on DIV12 (Figure $20 \mathrm{E}$ ). 
A
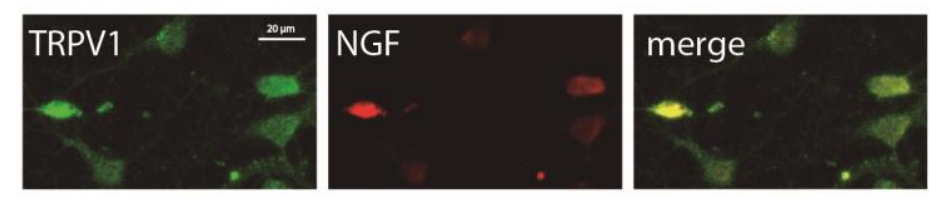

B

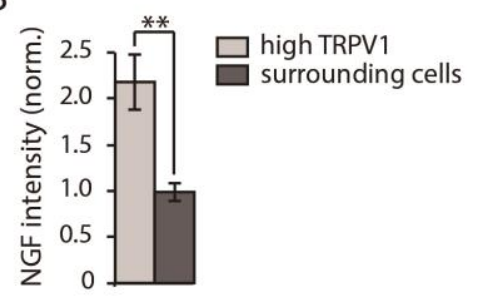

C
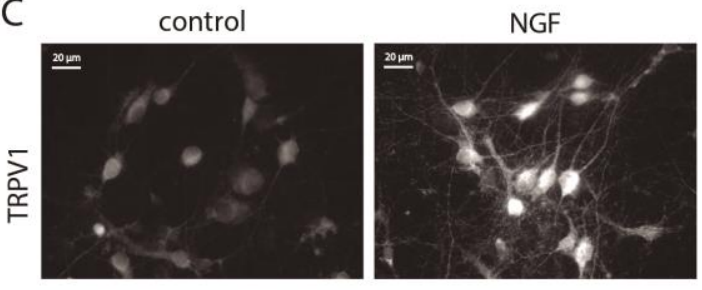

D
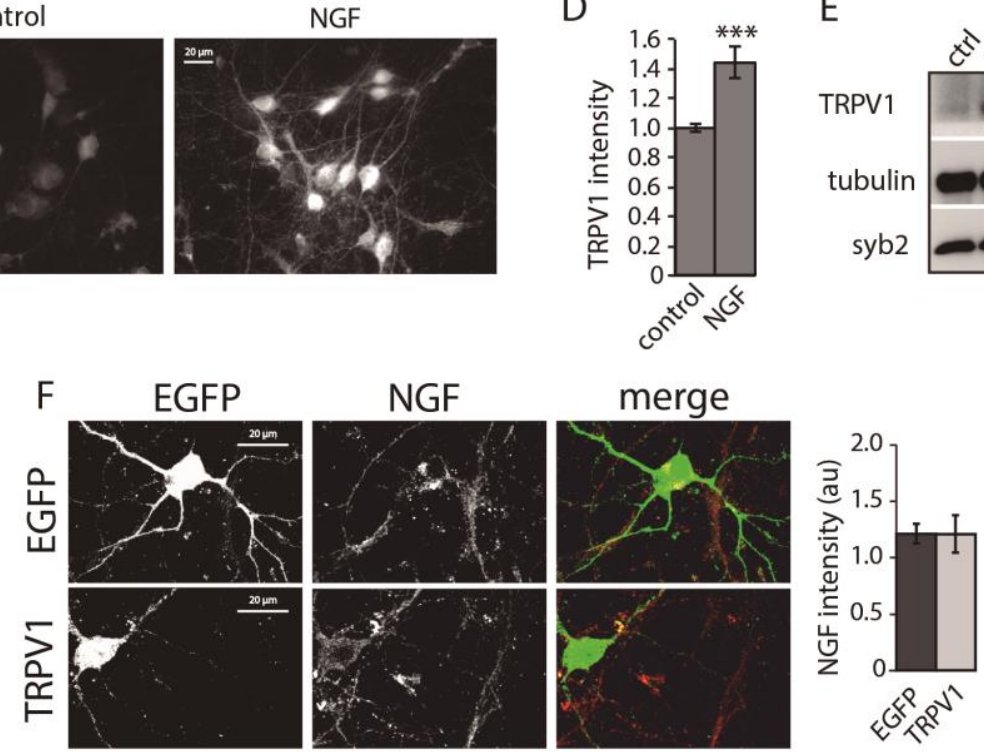

$E$

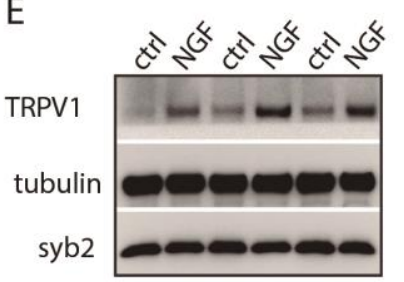

Figure 20. NGF promotes expression of TRPV1 in hippocampal neurons. A) ICC of rat hippocampal cell cultures for TRPV1 and NGF. NGF is highly expressed in somas of highexpressing TRPV1 hippocampal neurons, compared to surrounding neurons, quantified in (B) (values of fluorescence intensity of NGF in high-expressing nerons normalized to the average value of surrounding neurons (considered as 1); $n=15$ images from 3 different neuronal cutures). C) Treatment of hippocampal neurons with $1 \mathrm{ng} / \mathrm{ml}$ NGF at DIV12-13 overnight increases TRPV1 expression assayed by immunostaining, quantified in (D); Control $n=26, N G F n=12$; images obtained from 4-9 independent neuronal cultures; error = SEM; significance determined by unpaired Student's t-test ${ }^{*} p<0.05,{ }^{* *} p<0.01,{ }^{* * *} p<0.001$ and confirmed by Western blot $(E) ; 3$ repetitions are shown. F) TRPV1 transfection at DIV2 does not increase NGF expression in hippocampal neurons immunostained at DIV 12-13; $n=6$ images from 2 independent neuronal cultures. Scale bars $=20 \mu \mathrm{M}$. Experiments and immunostainings for $\mathbf{C}$ and analysis for $\mathbf{D}$ done in collaboration with Erika Avendaño Guzmán. Western blots for E performed by Dr. Saheeb Ahmed.

\subsubsection{High expression of TRPV1 in OLM neurons is driven by NGF}

NGF has long been reported to be expressed in brain and hippocampus (Gall and Isackson, 1989; Korsching et al., 1985). In the hippocampus NGF has been 


\section{RESULTS}

observed in subsets of inhibitory interneurons in multiple regions including neurons in the SO (Bizon et al., 1999; Pascual et al., 1998) that receive axonal projections from cholinergic septohippocampal neurons (Rocamora et al., 1996), i.e. OLM neurons, where we see high levels of TRPV1. Thus it is possible that NGF could drive TRPV1 expression in OLM neurons, since it is known that expression of TRPV1 in DRGs is regulated by NGF (Ernsberger, 2009). NGF has also been reported to be present in inhibitory neurons expressing TRPV1 in the human hippocampus (Sun et al., 2012) and to be necessary for proper maintenance of TRPV1 expression levels in nociceptors (Luo et al., 2007).

As shown in Figure 20 A high TRPV1-expressing OLM neurons also expressed high levels of NGF. Quantitation of immunofluorescence signal of NGF in high TRPV1 expressing neurons showed that NGF was always higher in these neurons than in surrounding neurons with lower TRPV1 levels (Figure $20 \mathrm{~B}$ ). TRPV1 and NGF are increased by the same amount in OLM neurons, possibly suggesting that the magnitude of TRPV1 expression directly depends on the amount of NGF expressed.

To assess the opposite scenario, if high TRPV1 expression would concomitantly upregulate the expression of NGF, we transfected hippocampal neurons in vitro at DIV12, but as shown in Figure $20 \mathrm{~F}$, TRPV1 overexpression in hippocampal neurons could not initiate NGF expression in any of the transfected cells.

In summary, these results show evidence that NGF can upregulate TRPV1 expression in most hippocampal neurons in early and late developmental stages in vitro. In a subset of hippocampal inhibitory interneurons, NGF is highly expressed and these neurons also have high levels of TRPV1. TRPV1 expression alone does not drive the expression of NGF in hippocampal neurons. 


\section{RESULTS}

\subsection{TRPV1 UPREGULATION UPON NEURONAL ACTIVATION: A LINK BETWEEN TRPV1 AND BDNF}

In DRG, BDNF is known to be expressed in a population of small- to medium-sized sensory neurons (Barakat-Walter, 1996; Merighi et al., 2008; Obata and Noguchi, 2006; Wetmore and Olson, 1995) including a population of TRPV1-positive cells (Xia et al., 2012) and NGF-responsive neurons (Apfel et al., 1996; Michael et al., 1997). BDNF has been associated with neuro-inflammatory pain (Xia et al., 2012; Zhao et al., 2006), hyperalgesia (Groth and Aanonsen, 2002), neuropathic pain (Fukuoka et al., 2001; Khan and Smith, 2015; Obata and Noguchi, 2006) and inflammation (Cho et al., 1997a, 1997b). In neurons from the DRG, NGF, besides driving TRPV1 expression (as mentioned in the last section) can also regulate expression of BDNF (Apfel et al., 1996; Merighi et al., 2008; Michael et al., 1997). Similar to TRPV1, corresponding with the fact that BDNF is jointly upregulated during inflammation (Cho et al., 1997a, 1997b), axotomy (Michael et al., 1997; Tonra et al., 1998), hyperalgesia (Yajima et al., 2002) and neuro-inflammatory pain (Xia et al., 2012). In the neuronal pain model, BDNF is known for its pronociceptive properties (reviewed in Khan and Smith, 2015) possibly by decreasing desensitization of TRPV1 channels (Ciobanu et al., 2009; Obata and Noguchi, 2006). Activation of TRPV1 on the other hand has been reported to cause an increase in BDNF release (Lever et al., 2001). Although one study suggested that BDNF increases TRPV1 expression in DRG cells (Ciobanu et al., 2009), the potential control of TRPV1 expression by BDNF remains uncertain (Price et al., 2005). In the hippocampus, to our knowledge, there has not been any study linking TRPV1 expression and/or activation with BDNF. 


\subsubsection{TRPV1 overexpression increases BDNF}

A
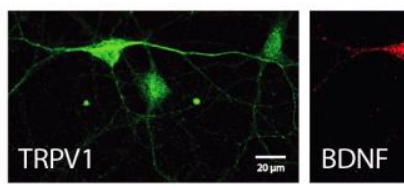

BDNF

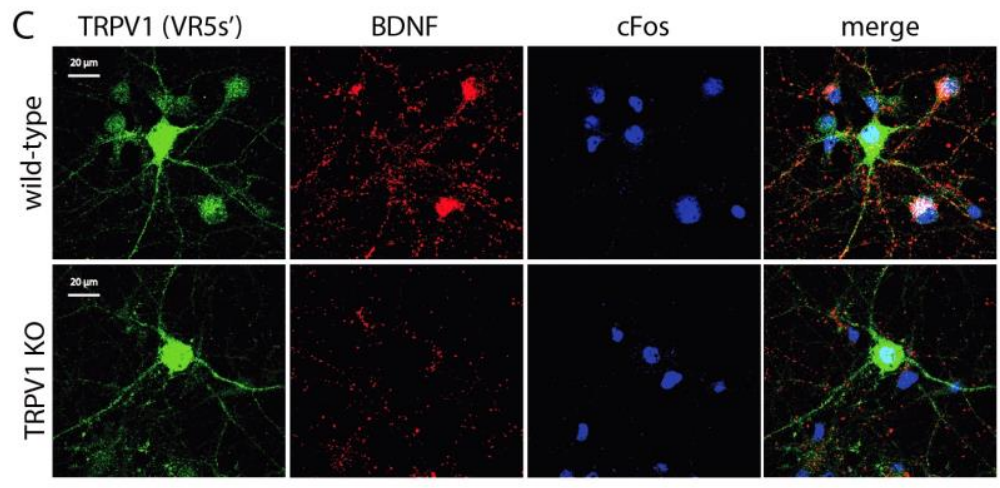

E

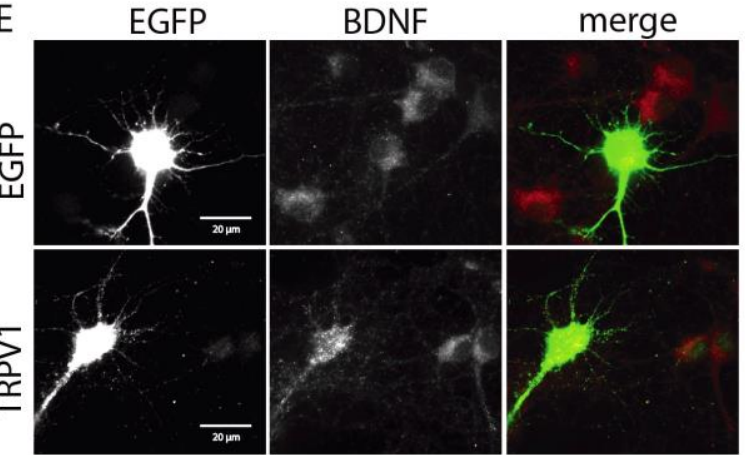

B

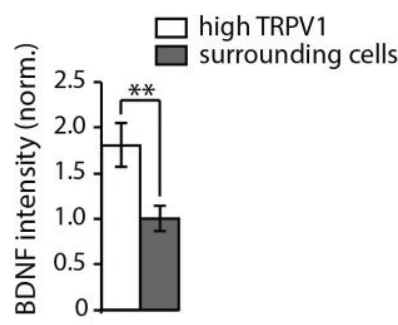

D
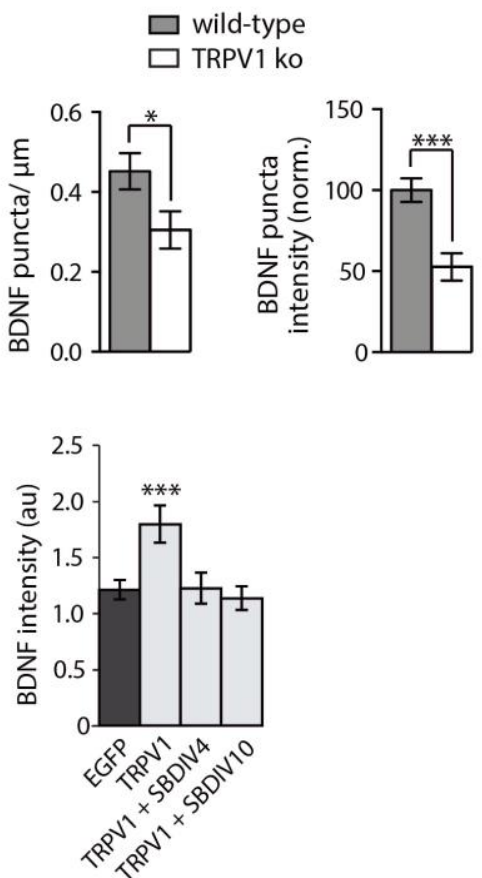

Figure 21. High TRPV1 expression correlates with increased BDNF levels in hippocampal neurons. A) ICC of rat hippocampal cell cultures immunostained with TRPV1 and BDNF. B) High TRPV1 expressing neurons have twice as much BDNF signal as surrounding neurons (values for high TRPV1-expressing neurons normalized to the average fluorescent intensity of neurons surrounding it; $n=24$ images). C) High-expressing TRPV1 mouse neurons have increased BDNF on neuronal processes (quantified in $\mathbf{D}$, left panel, as the number of BDNF-positive puncta/ $\mu \mathrm{m}$ ), compared to TRPV1 knockout neurons, identified by the remaining signal from the C-terminus TRPV1 antibody (Millipore). The BDNF signal intensity of analyzed puncta was $50 \%$ lower in TRPV1 KO neurons compared to WT (D, right panel) (WT and TRPV1 KO $n=9$ ). E) Transfection on DIV2 with TRPV1 increases BDNF in transfected neurons immunostained at DIV14. This increase in BDNF is blocked by $1 \mu \mathrm{M}$ SB treatment at DIV4 and DIV10 (EGFP $n=18$, TRPV1 DIV2 $n=21$, TRPV1 DIV2 + SB $1 \mu \mathrm{M}$ DIV4 $\mathrm{n}=9$, TRPV1 DIV2 + SB $1 \mu \mathrm{M}$ DIV10 $\mathrm{n}=12$; images taken from 3-7 independent neuronal cell cultures; error = SEM; significance determined by Student's unpaired $\mathrm{t}-$ test $\left.{ }^{*} p<0.05,{ }^{* *} p<0.01,{ }^{* * *} p<0.001\right)$. Scale bars $=20 \mu \mathrm{m}$. 


\section{RESULTS}

Activation of TRPV1 channels overexpressed in hippocampal neurons could potentially lead to hyperactivity of the transfected neuron. Therefore, we were interested if TRPV1 transfection in hippocampal neurons at DIV2 and DIV10 would lead to an increase in neuronal activity that was detected using antibodies against BDNF, which is known to be upregulated by neuronal activity (Patterson et al., 1992; Zafra et al., 1990). As shown in Figure $21 \mathrm{E}$, when TRPV1 was transfected before the main period of synaptogenesis, an increase in the detected signal for BDNF was observed when compared to surrounding cells and to EGFP transfected neurons. When we treated transfected neurons with the TRPV1-specific antagonist SB either two or eight days after transfection, the BDNF augmentation was reverted, suggesting that TRPV1 overexpression and activation account for a rise in neuronal activity and an increase in BDNF (Figure $21 \mathrm{E}$; right panel).

\subsubsection{High TRPV1-expressing neurons have increased BDNF expression}

When we found that a population of hippocampal inhibitory interneurons highly expresses the TRPV1 channel, we were interested if this neuron population would have augmented levels of BDNF, as TRPV1-transfected neurons do. As depicted in Figure $21 \mathrm{~A}$ and $\mathrm{B}$, hippocampal neurons in vitro with increased TRPV1 expression showed, on average, greater levels of BDNF expression compared to lower-expressing TRPV1 neurons. To test if TRPV1 correlates with a rise in BDNF expression, we compared OLM neurons from mouse WT vs TRPV1 KO cell cultures. Interestingly, the detection pattern obtained by the BDNF antibody in mouse cultures was different than in rat cultures. While in rat cultures the localization of BDNF was somatic, in mouse cultures the detected signal was punctate, distributed in what appear to be terminals contacting the high TRPV1expressing neuron (Figure $21 \mathrm{C}$ and $\mathrm{D}$ ). On average, the quantitation showed that in TRPV1 KO conditions (where TRPV1-expressing neurons are detected by low signal from the C-T antibody) this subpopulation of neurons had significantly less puncta per micron with half the fluorescence intensity of WT neurons (Figure $21 \mathrm{D}$ ). 


\subsubsection{Elevated neuronal activity increases both TRPV1 and BDNF levels}

As mentioned before, neuronal activity drives expression of BDNF, and in the PNS TRPV1 and BDNF expression are often upregulated under similar conditions. We aimed to detect if TRPV1, like BDNF, would also be upregulated after an elevation in neuronal activity. We used $50 \mu \mathrm{M}$ forskolin in order to raise cAMP levels and subsequent neuronal activity in cultured hippocampal neurons. As expected, $50 \mu \mathrm{M}$ forskolin boosted BDNF and cFos levels when applied to DIV3 as well as to DIV10 rat hippocampal cultures (Figure 22 and Figure 23). Fluorescence intensity of the TRPV1 C-T antibody was concomitantly higher in samples treated with forskolin.
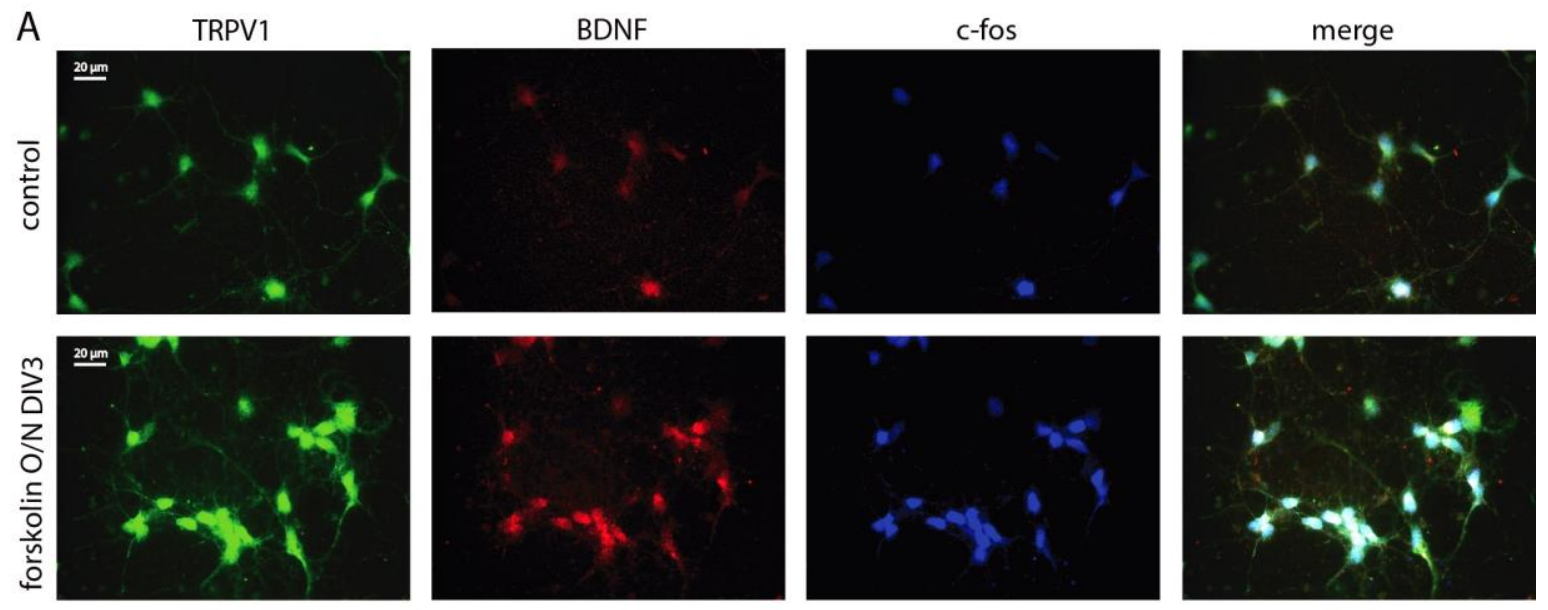

B
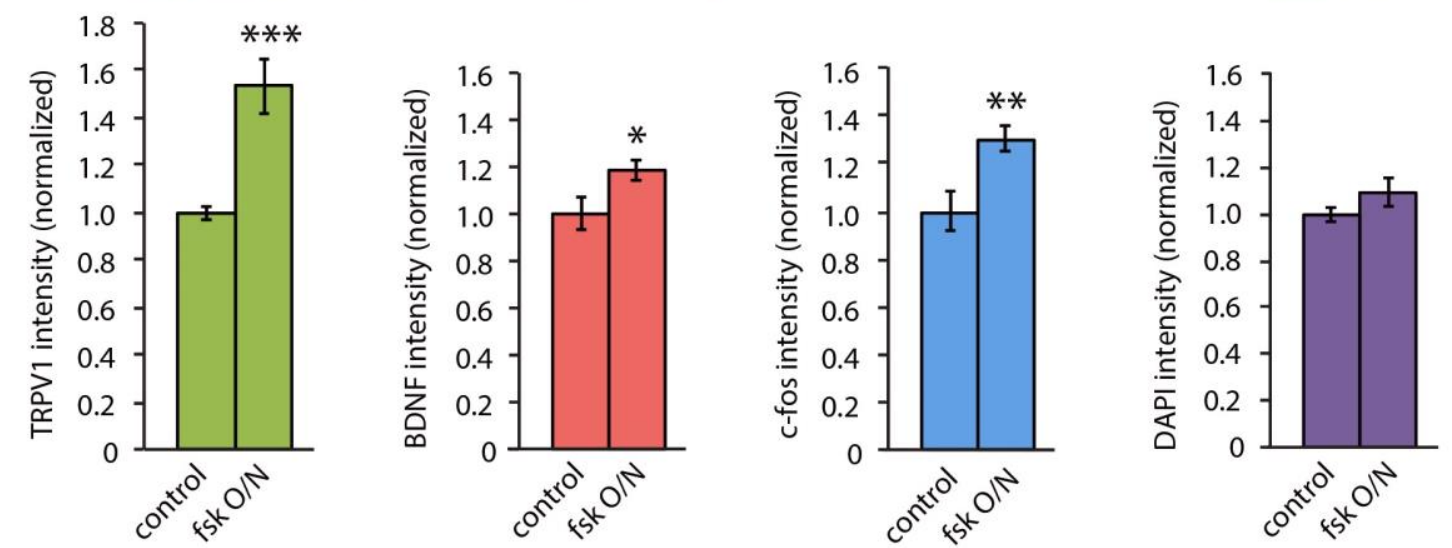

Figure 22. TRPV1 expression is increased by neuronal activity in hippocampal neurons. A) ICC of TRPV1, BDNF, and c-fos at DIV3 ; $50 \mu \mathrm{M}$ forskolin treatment overnight increases TRPV1, BDNF and cFos signal (scale bar $=20 \mu \mathrm{m}$ ), quantified in (B) (control $n=20$, forskolin $n=15$ images acquired from 5-7 separate neuronal cultures; error = SEM; significance determined by unpaired Student's t-test ${ }^{*} p<0.05,{ }^{* *} p<0.01,{ }^{* *} p<0.001$ ). 


\section{RESULTS}

Contrary to these results, quantified TRPV1 protein levels detected with the TRPV1 $\mathrm{N}-\mathrm{T}$ antibody by WB remained unchanged upon $50 \mu \mathrm{M}$ forskolin treatment.

When we used $10 \mu \mathrm{M}$ bicuculline as an alternative way to raise neuronal activity by blocking GABAa receptors, hence increasing excitation, in DIV10 cultured hippocampal neurons, we found that BDNF, cFos as well as the TRPV1 fluorescence signal was higher than in untreated samples (Figure 23). Nevertheless, the elevation of these three markers was not as widespread as in forskolin treatments, where it was observed in most of the analyzed cells (Figure 22 for DIV3 and Figure 23 for DIV10). In the presence of bicuculline some cells had a brighter immunofluorescence signal than others. The expected change in protein levels for TRPV1 was, however, not able to be detected with WB (data not shown).

Together, these results suggest that an increase in neuronal activity (revealed by an elevation in BDNF and cFos) ultimately led to an increase in levels of TRPV1 detected by immunofluorescence. TRPV1 endogenous expression or overexpression was correlated with BDNF suggesting that TRPV1 activation can increase neuronal activity. Still it remains uncertain how upregulation of TRPV1 after the induction of neuronal network activity occurs and if changes in TRPV1 expression would account for tuning activity in hippocampal neurons. 

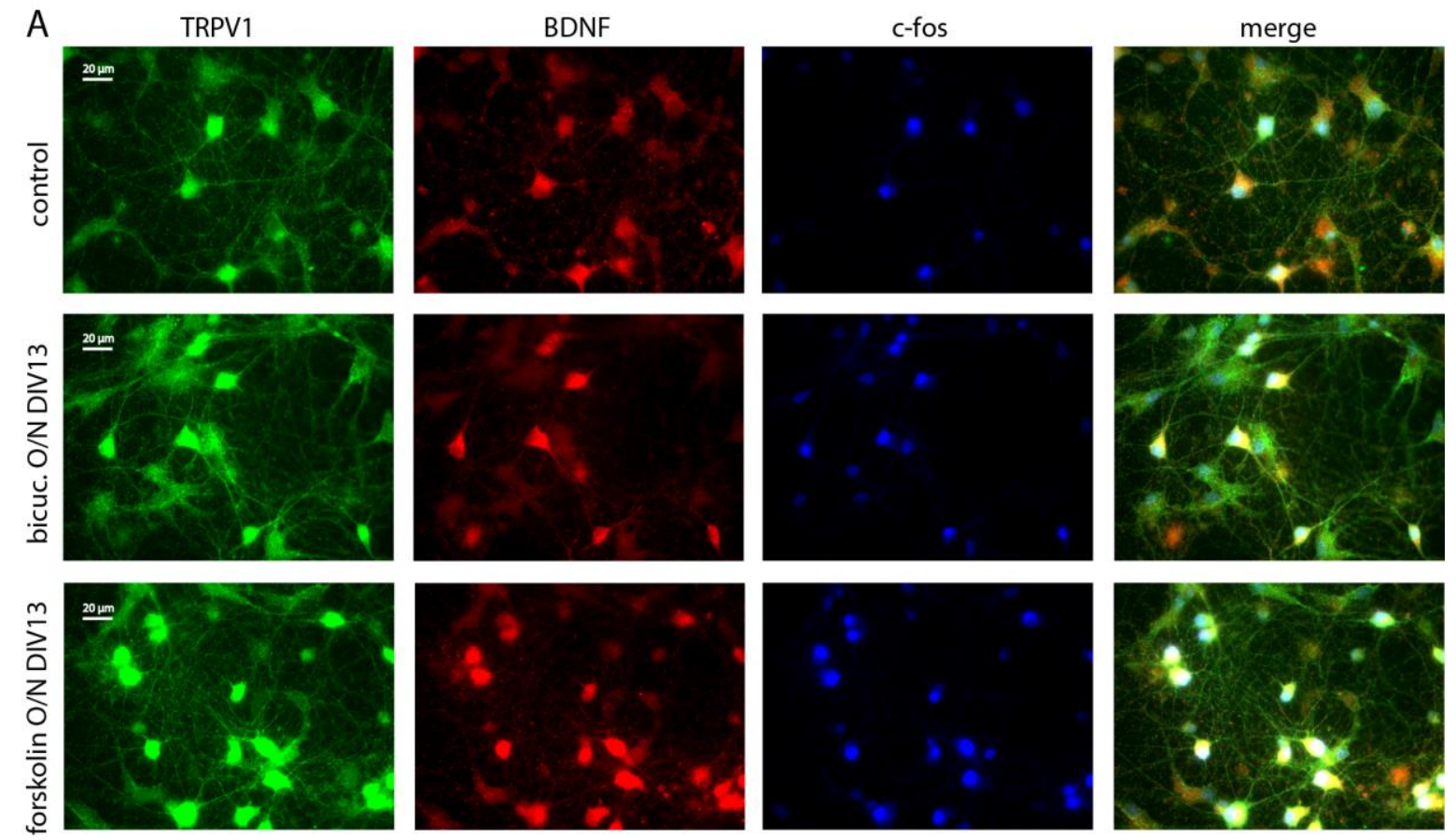

B
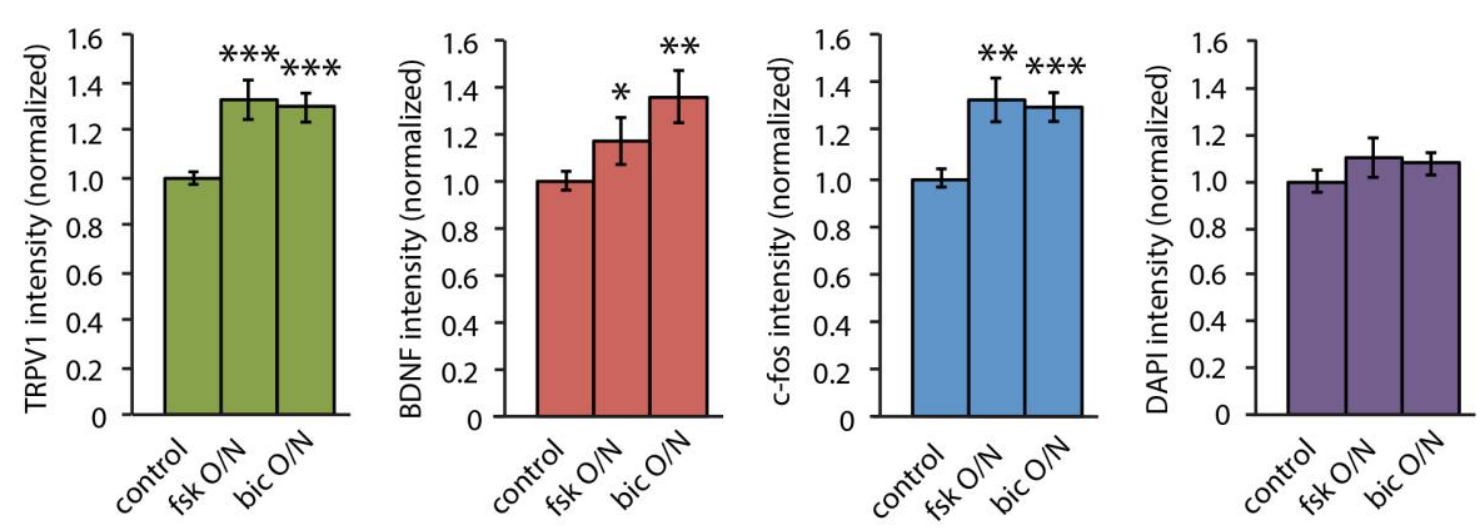

Figure 23. TRPV1 expression is increased by neuronal activity in hippocampal neurons. A) ICC of TRPV1, BDNF, and c-fos at DIV13;50 $\mu \mathrm{M}$ forskolin or $10 \mu \mathrm{M}$ bicuculline treatment overnight increases TRPV1, BDNF and cFos signal (scale bar $=20 \mu \mathrm{m}$ ), quantified in (B) (control $n=24$, forskolin $n=19$, bicuculline $n=20$; images acquired from 5-8 separate neuronal cultures; error = SEM; significance determined by unpaired Student's t-test ${ }^{*} p<0.05,{ }^{* *} p<0.01,{ }^{* * *} p<0.001$ ). Experiments and immunostainings for $\mathbf{A}$ and analysis for $\mathbf{B}$ done in collaboration with Erika Avendaño Guzmán. 


\subsection{DOES BDNF INCREASE EXCITATORY INNERVATION UPON HIGH TRPV1 EXPRESSION?}

Since the early nineties, there has been increasing evidence that neuronal activity has a pivotal role in synaptic transmission that can ultimately lead to changes in the number and strength of synapses (Bliss and Collingridge, 1993; Linden, 1994; Malenka and Nicoll, 1999). Neuronal activity also promotes BDNF expression and secretion (Patterson et al., 1992; Zafra et al., 1990) especially at synapses where it contributes to an enhancement in synaptic transmission (Bonhoeffer, 1996; Katz and Shatz, 1996; Thoenen, 1995). In excitatory synapses BDNF release can also occur from the post- to the pre-synaptic part, thus serving as a retrograde messenger modulating pre-synaptic neurotransmitter release (Hartmann, 2001; Matsuda et al., 2009). This background allowed us to hypothesize that if high TRPV1 expressing neurons are positive for concomitant high levels of BDNF, this neurotrophin could be acting as a retrograde messenger provoking the proliferation of excitatory innervation terminating on high TRPV1-expressing neurons and TRPV1-transfected hippocampal neurons at DIV2.

\subsubsection{BDNF controls excitatory presynaptic strength but not excitatory innervation to TRPV1 transfected neurons.}

As documented in section 4.4, heterologous TRPV1 expression on DIV2 leads to an increase in vGluT1 antibody signal (and co-localizing Sy1u fluorescence intensity) as wells as in excitatory synapse number contacting TRPV1overexpressing neurons (Figure 16). TRPV1 overexpression at DIV2 enhances neuronal activity (indicated by BDNF levels), but BDNF could differentially alter synaptic strength depending on the day at which TRPV1 was transfected. Using the BDNF TrKB-lgG scavenger to prevent secreted BDNF from reaching endogenous TrkB receptors, we tested if changes in excitatory presynaptic strength caused by TRPV1 overexpression depended on secreted BDNF. 
A
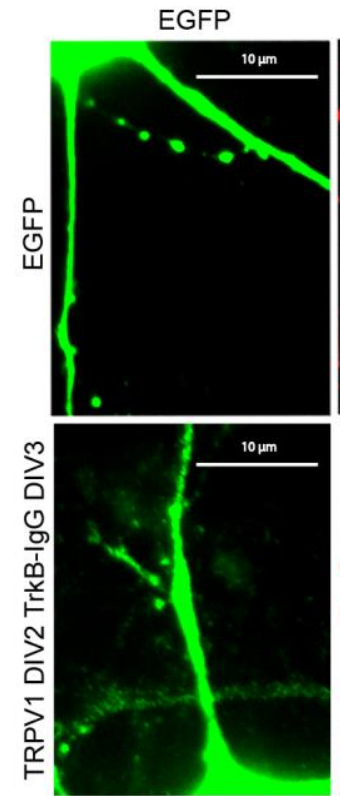

B

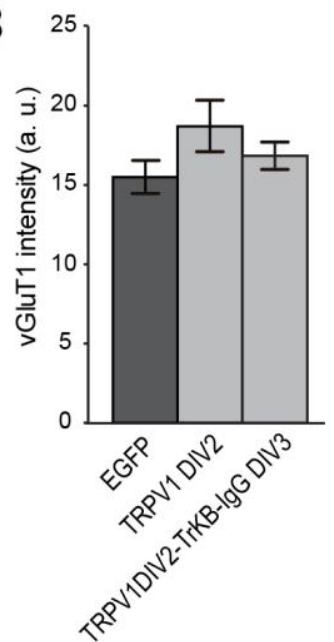

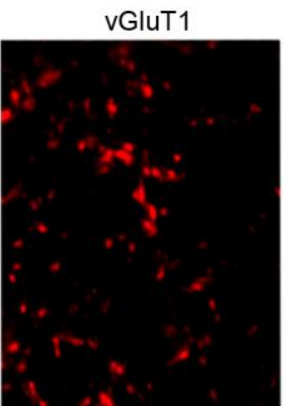
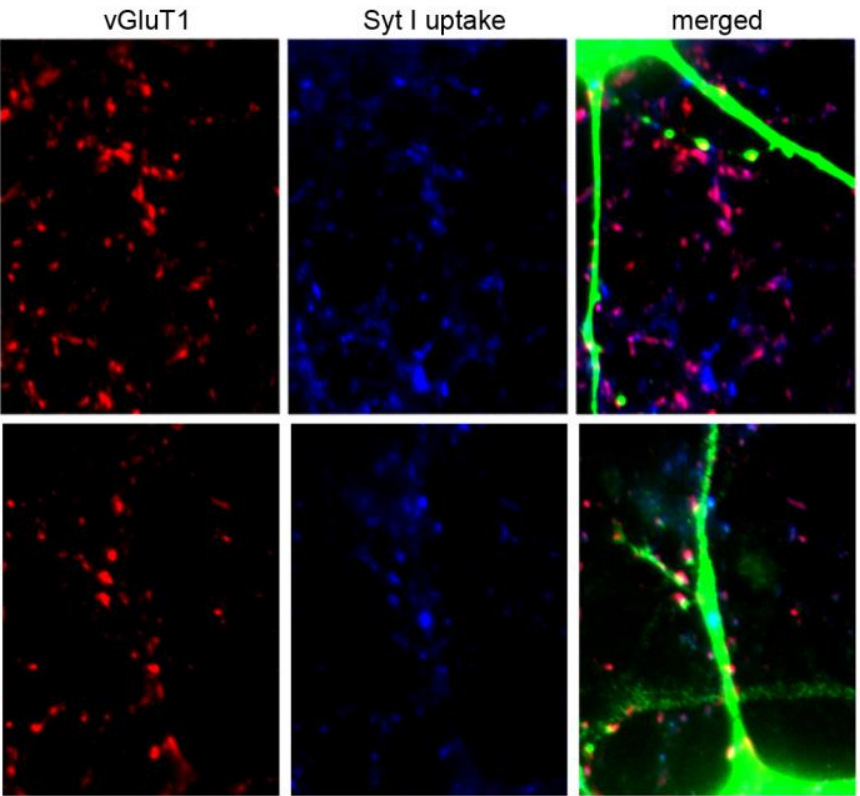

C

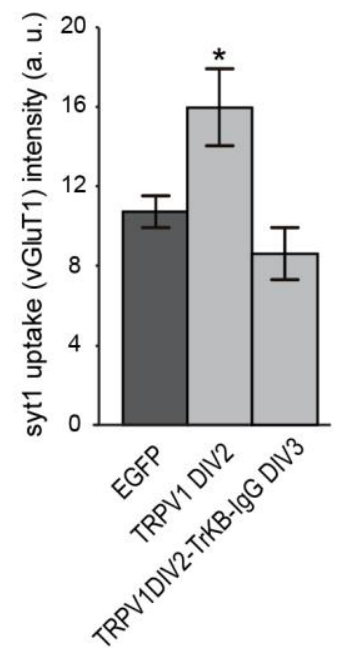

D

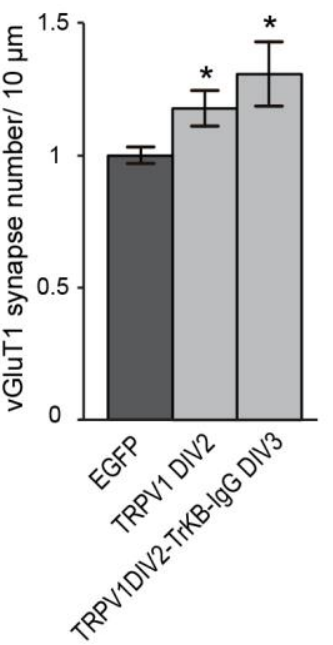

Figure 24. TRPV1 overexpression in neurons increases excitatory innervation through a BDNF-independent mechanism. A) Triple ICC for vGluT1 and syt1u of rat hippocampal neurons transfected with TRPV1-IRES-GFP or EGFP before synapse formation on DIV2. B) Quantitation of the excitatory synaptic strength, assayed by vGluT1 signal and overlapping syt1u fluorescence intensity (C) D) Quantitation of excitatory synapse number (number of vGluT1 puncta) on TRPV1expressing cells with and without treatment with $100 \mathrm{ng} / \mathrm{ml}$ TrKB-IgG at DIV3 compared to EGFP (Images: EGFP; $n=60$, TRPV1; $n=30$, TRPV1 + TrKB-lgG; $n=12$ images obtained from 4-20 different neuronal cultures; all values normalized to the average value obtained for EGFP transfected neurons; error = SEM; significance determined by Student's unpaired t-test * $p<0.05$, ** $p<0.01,{ }^{* *} p<0.001$; synapse number values normalized to the average value obtained for EGFP transfected neurons). 


\section{RESULTS}

Neurons overexpressing TRPV1 at DIV2 were treated with $100 \mathrm{ng} / \mathrm{ml}$ TrkB-lgG scavenger at DIV3 and incubated for 10 days before fixation. The observed effects of TRPV1 overexpression on excitatory presynaptic strength, identified by the amount of fluorescent signal of Syt1u on vGluT1 stained synaptic boutons, was reverted to a level similar to EGFP-transfected control neurons (Figure $24 \mathrm{~A}$ and C). On the other hand, the TrkB-IgG scavenger did not prevent the formation of excess excitatory synapses onto TRPV1 transfected neurons; quite the contrary, it tended to slightly increase the quantity of glutamatergic synapses (Figure $24 \mathrm{~A}$ and D).

\subsubsection{BDNF does not regulate excitatory or inhibitory innervation in OLM neurons}

As mentioned in section 4.3, in hippocampal neuronal cultures, neurons highly expressing TRPV1, most likely corresponding to OLM neurons had higher BDNF levels compared to other neurons. We also found that BDNF produced changes in glutamate release caused by early TRPV1 overexpression. In addition BDNF has been postulated to be released from post-synaptic regions in order to retrogradely regulate pre-synaptic strength (see section 2.7.2). Therefore it is imaginable that BDNF could be responsible for changes observed in pre-synaptic strength of excitatory synapses onto OLM neurons. To test this we added $100 \mathrm{ng} / \mathrm{ml}$ TrkB-lgG scavenger to hippocampal neuronal rat cultures at DIV2 and fixed them at DIV13 for imaging. As shown in Figure 25 we did not see any changes in the number of individual glutamatergic inputs positive for vGluT1 nor in the average fluorescence intensity of vGluT1 puncta in OLM neurons in TrkB-lgG treated cell cultures compared to untreated samples. 

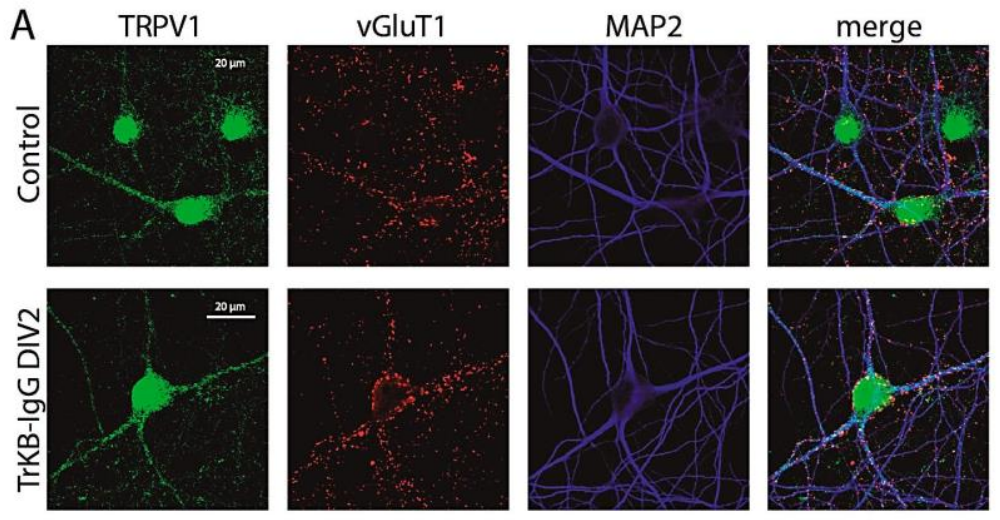

B
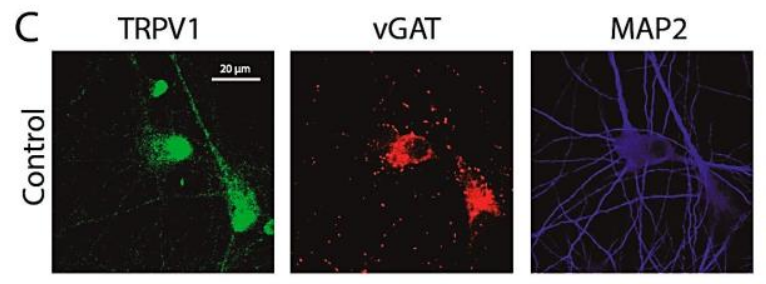

merge
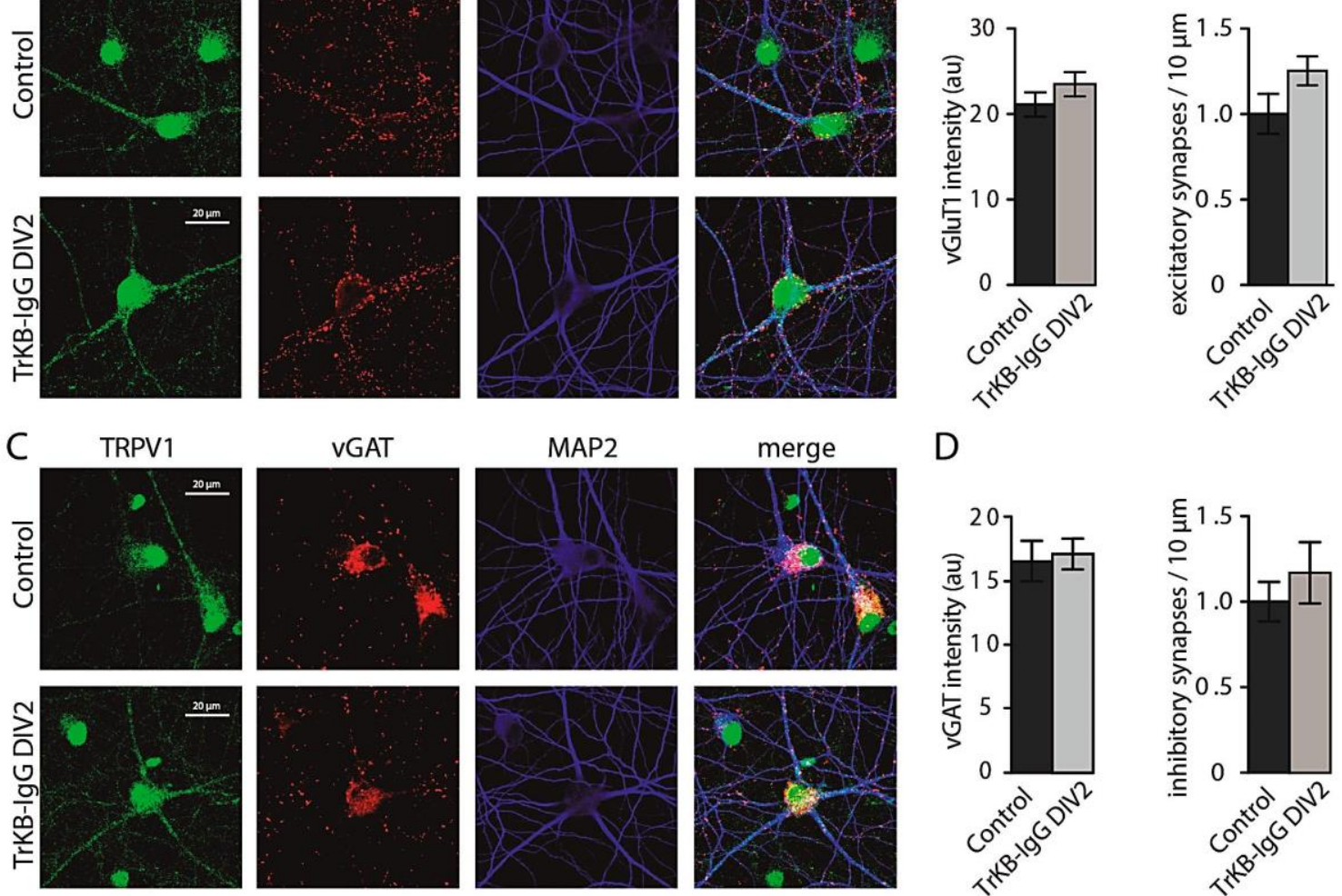

D
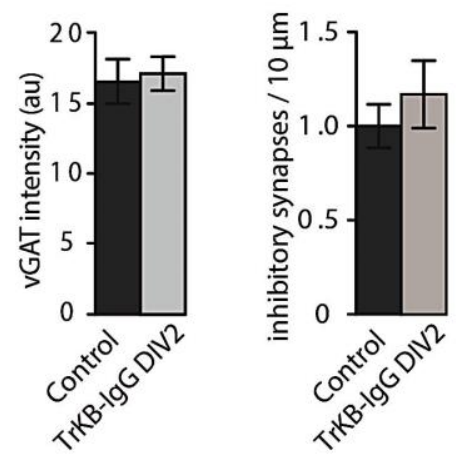

Figure 25. BDNF is not required for increased excitatory and inhibitory innervation of high TRPV1 expressing neurons. A) ICC of DIV14 rat neurons for TRPV1, vGluT1 and MAP2, or with TRPV1, vGAT and MAP2 (C). Treatment with $100 \mathrm{ng} / \mathrm{ml}$ TrkB-lgG scavenger at DIV 2 (fixed DIV13) did not significantly alter the number or intensity of of vGluT1 (B) or VGAT (D) positive terminals contacting neurons highly expressing TRPV1 $(n=12$ images each for vGluT1 and vGAT in control and TrKB-IgG treated cells of 3 different neuronal cultures ; error = SEM; significance determined by unpaired Student's t-test ${ }^{*} p<0.05,{ }^{* *} p<0.01,{ }^{* * *} p<0.001$ ).

\subsection{TRPV1 REPORTER MOUSE}

\subsubsection{The cre-lox $P$ system}

The mechanism of gene expression in the coliphage P1 known as the cre-lox $P$ system basically consists of the recombinase enzyme cre that will only catalyze homologous recombination at a 34 bp DNA sequence known as lox $P$ (Abremski and Hoess, 1984; Hoess et al., 1982; Sauer, 1987; Sauer and Henderson, 1988; 


\section{RESULTS}

Sternberg and Hamilton, 1981). Hence this system would allow a "sequencespecific" recombination of a region of interest flanked by lox $P$. This genetic tool has made possible the generation of animal models carrying these sequences at specific sites in their genome, which permits control of conditional (cre dependent) targeting of specific gene sequences (lox $P$ flanking) (Orban et al., 1992), thus allowing spatial and temporal modifications of the expression of a desired gene.

Ideally the cre sequence would be present downstream of the sequence of a desired gene, the expression of which will be controlled by its intrinsic promoter, whereas the sequence to be modified should be flanked by lox P. Depending on the orientation and position in the chromosome of the lox $\mathrm{P}$ sequence, the resulting modification could lead to a "knock out" or deletion, translocation or inversion of the target gene (Bouabe and Okkenhaug, 2013; Gu et al., 1994; Orban et al., 1992).

Using cre-lox $P$ mouse lines, the creation of cell-specific as well as developmentaltimed reporter mouse lines have become easily accessible. Unlike conventional knock in reporter mice, the flexibility of this system allows the generation of multiple mutants using a single cre or lox $P$ line. To date there is already an extensive list of mouse lines for different promoters driving cre expression as well as of targeted floxed DNA sequences. A large database of cre and lox $\mathrm{P}$ transgenic mice have recently become available (reviewed in Bouabe and Okkenhaug, 2013).

Because of the wide variety of neuronal cell types in the brain, in neurobiology one of the purposes for using these lines is to generate reporter mice for specific neuronal populations. This can be achieved by crossing cre lines targeting proteins that are expressed in confined cellular populations with lox $P$ lines that would allow the expression of reporter fluorescent molecules upon cre activity (reviewed in (Abe and Fujimori, 2013).

Despite the progress achieved using this system and the large number of specific conditional mutant mouse lines developed, recent studies have unveiled a series of 


\section{RESULTS}

unwanted issues and limitations that could occur if certain considerations are not attended to during the generation of a cre-lox $P$ line (Heffner et al., 2012; Matthaei, 2007; Schmidt-Supprian and Rajewsky, 2007). Some transgenic mouse strains have been reported to have a number of unexpected or undesired phenotypes that were not initially evident (Balordi and Fishell, 2007; Eckardt et al., 2004; Heffner et al., 2012; Kobayashi and Hensch, 2013; Lu et al., 2008; Means et al., 2005; Pilon et al., 2008; Weng et al., 2008). Thus it is recommended that results obtained using this system need to be carefully controlled, and it is of paramount importance that each research group characterizes the transgenic model themselves in order to avoid possible unknown latent side effects of any employed strain.

\subsubsection{The TRPV1-tdTomato reporter mouse line}

As mentioned in section 4.2 we were interested in localizing the expression of TRPV1 in the hippocampus. We used various methods to conclude that functional TRPV1 channels are expressed in hippocampal neurons. By using immunohistoand cyto-chemical assays we found TRPV1 protein expression in the hippocampus of adult mice is confined to a subset of inhibitory interneurons in the stratum oriens; immunolabeling against different neuronal markers allowed us to determine that such neurons corresponded to OLM neurons.

Despite the extensive use of immunohisto- and cyto-chemistry for the cellular and subcellular localization of a myriad of cell-specific proteins, this method is known to have limitations including non-specific staining, that can ultimately lead to unreliable results and misinterpretation (de Matos et al., 2010; Ward and Rehg, 2014; Yaziji and Barry, 2006). Such limitations can be tackled by the use of a reporter mouse strain to induce the conditional expression of a reporter gene (e.g. tdTomato) driven by a specific promoter. In an attempt to identify the expression pattern of TRPV1 in the hippocampus a TRPV1-cre mouse line has been recently generated and commercialized. Using the cre-lox P model, Cavanaugh et al., 2011 


\section{RESULTS}

concluded that in the hippocampus TRPV1 expression is restricted exclusively to Cajal-Retzius cells expressing reelin.

To corroborate this result and our findings using immune-histochemical procedures we bred a transgenic reporter mouse by crossing the TRPV1-Cre (Cavanaugh et al., 2011) with a "cre-responding" Rosa26 tomato mouse line (Madisen et al., $2010 b$ ) that has lox $P$ sequences flanking a stop cassette (downstream of a CAG promoter) that prevents the transcription of the red fluorescent protein tdTomato. In the resulting offspring, the expression of tdTomato should be limited to cells expressing TRPV1.

\subsubsection{Expression pattern of tdTomato signal in the F1 TRPV1- tdTomato transgenic mouse}

As mentioned above, we bred a TRPV1 reporter mouse line that would conditionally express tdTomato under control of the TRPV1 promoter. Since we were interested in testing if TRPV1 is expressed in the hippocampus, we prepared brain slices from F1 animals. Before slices were obtained we genotyped each mouse to ensure they were TRPV1-Cre-Rosa26-tdTomato positive.

Using the F1 TRPV1 reporter mouse we detected high expression of tdTomato fluorescence in regions corresponding to the piriform cortex, caudate putamen, subthalamic nucleus, anterior hypothalamic area, posterior hypothalamic nucleus, lateral amygdaloid nucleus, perirhinal cortex and hippocampus (see Figure 26 and Figure 27). In the hippocampus a strong fluorescence was detected in the lacunosum moleculare layer, radiatum layer, dentate gyrus, fascicola cinereum, and in the stratum oriens only a small number of neurons was found to be positive for td-Tomato. 


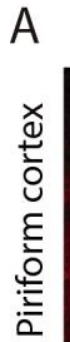

tdTomato

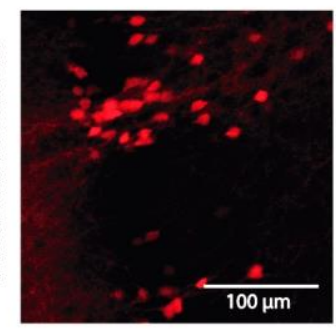

B

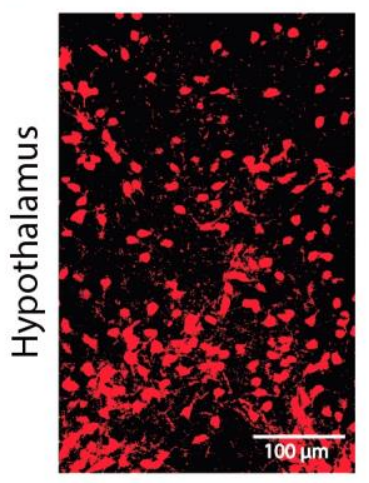

TRPV1 C-T (Neuromics)

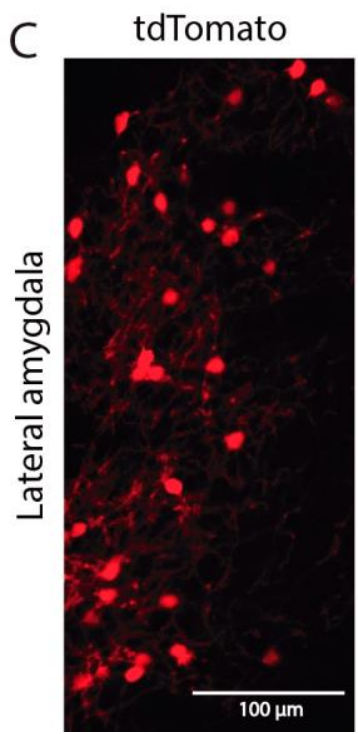

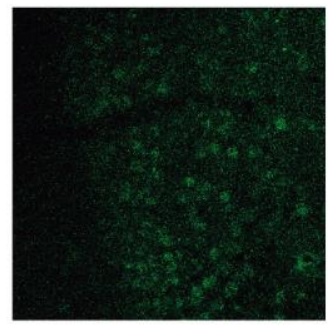

TRPV1 C-T (Millipore)

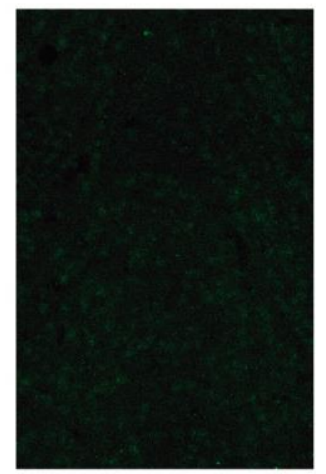

DAPI
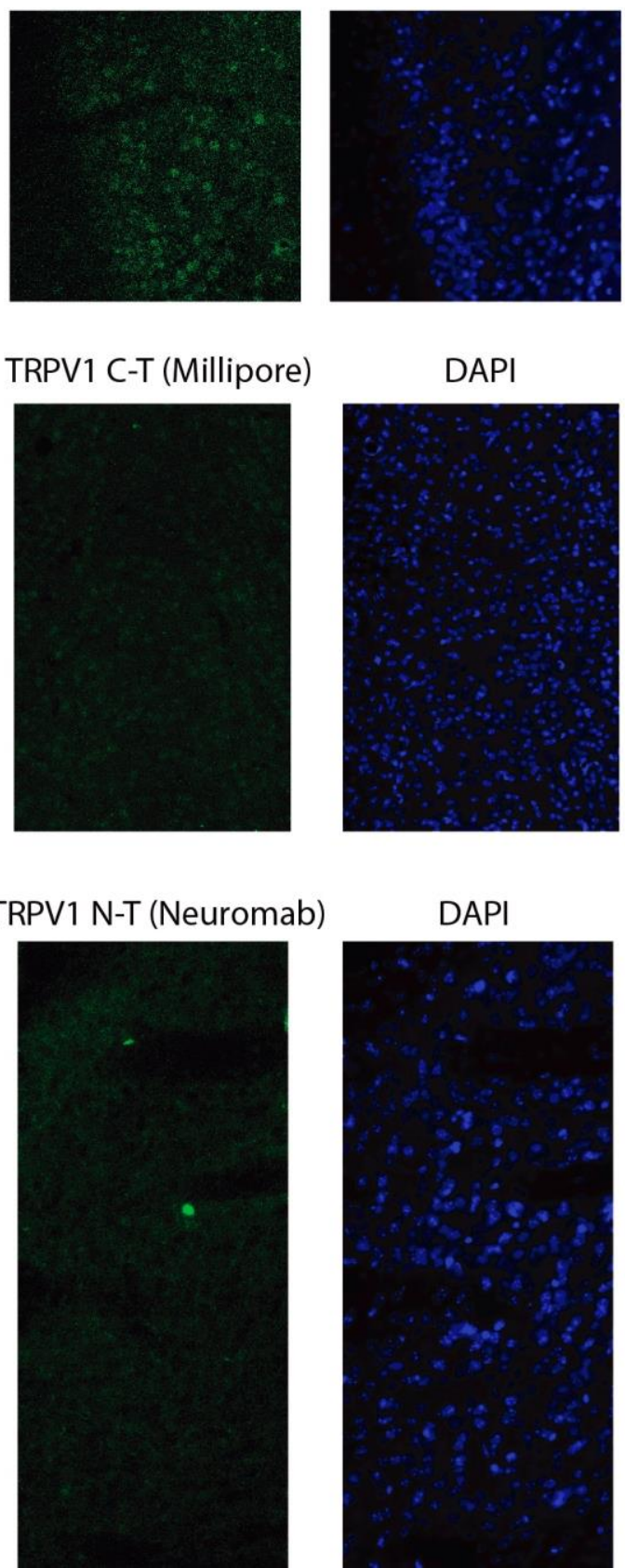

DAPI

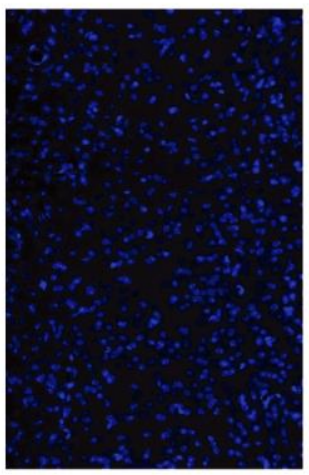

DAPI

merge

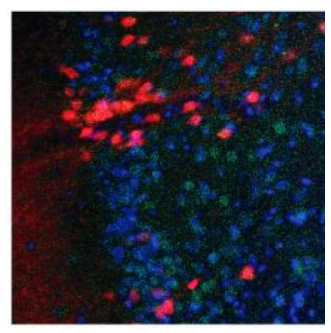

merge

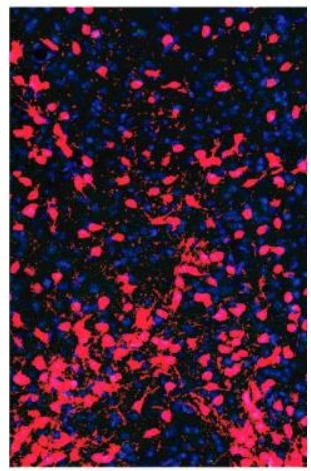

merge
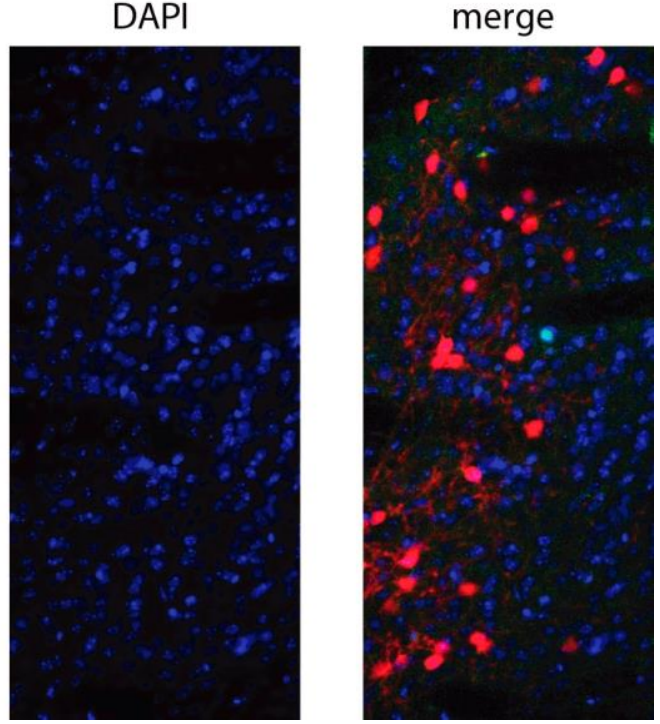

Figure 26. tdTomato expression pattern in brain in the TRPV1cre X Rosa26-tdTomato reporter mouse line. Representative images of three different brain areas having tdTomato signal; for every brain region an example of each antibody it is shown. (A) IHC shows expression of the tdTomato reporter gene in the piriform cortex, where the C-T (Neuromics) antibody does not colocalize with the tdTomato-positive neurons. B) In the Hypothalamus the C-T antibody (Millipore) also failed to detect the neurons labeled with the tdTomato signal. C) The N-T antibody (Neuromab) did not colocalize with the tdTomato-positive neurons in the lateral amygdala. For each representative image $n=3$ independent immunostainings were performed for each antibody. 
We compared the signal provided by tdTomato to that given by $\mathrm{HC}$ using three commercial antibodies specific for TRPV1. Surprisingly the three commercial TRPV1 antibodies against the $\mathrm{C}$ or N-T epitope provided an immunofluorescent signal with a distribution that did not completely match that of tdTomato.

For a more detailed comparison, we examined four areas of the brain at high magnification that had the most prominent expression of tdTomato (i.e. the hippocampus, hypothalamic area, amygdaloid nucleus and piriform cortex).
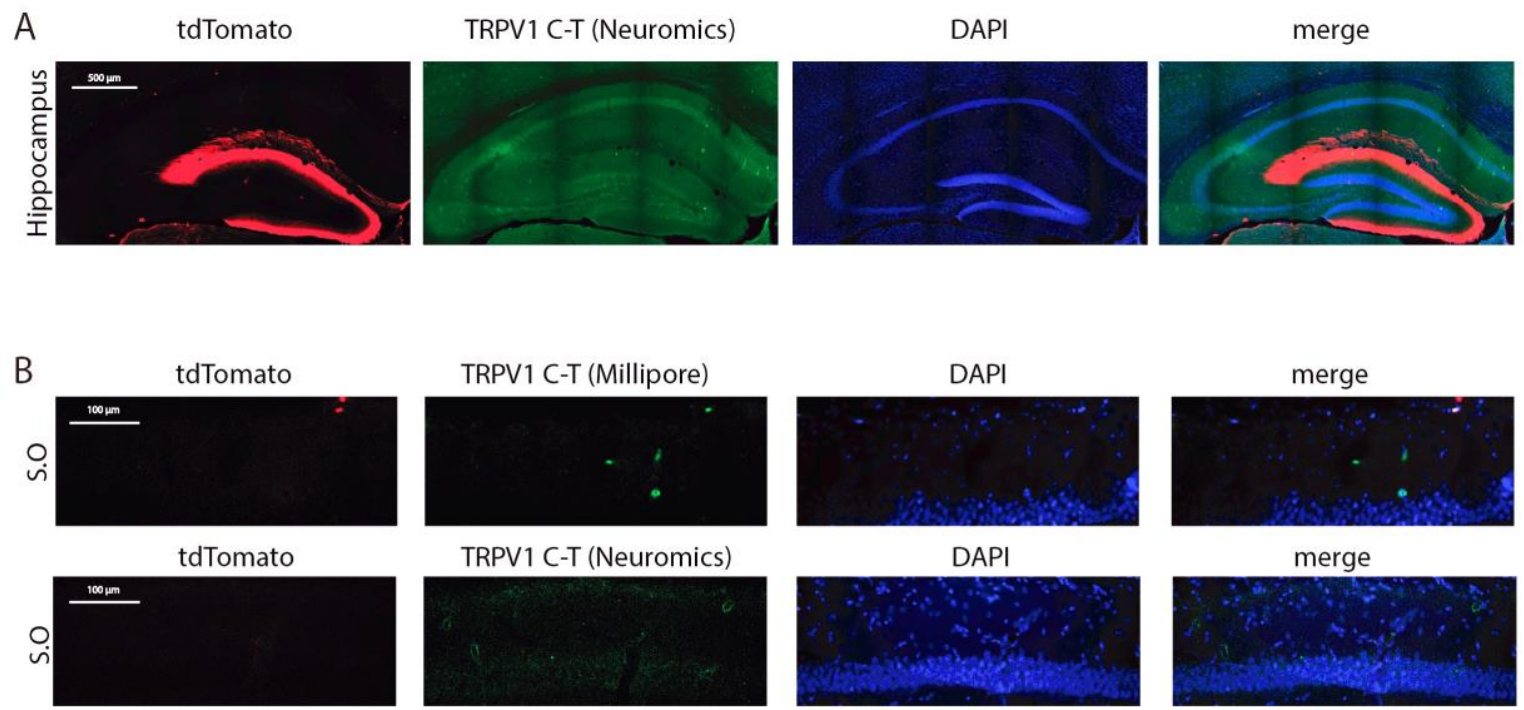

TRPV1 C-T (Neuromics)

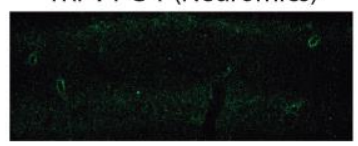

DAPI
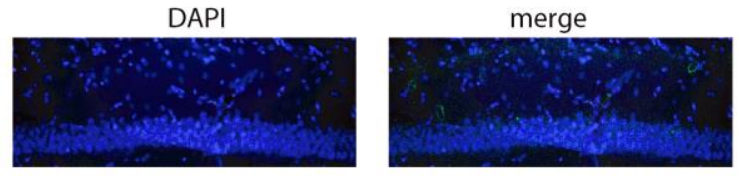

tdTomato

TRPV1 N-T (Neuromab)
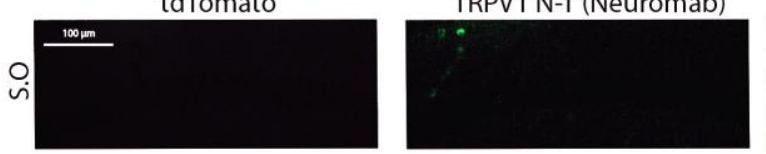

DAPI

merge
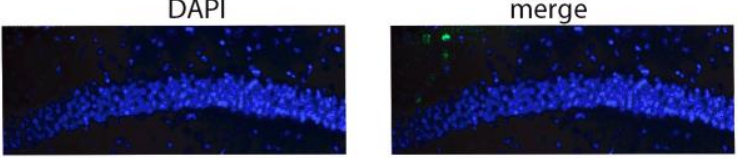

Figure 27. Hippocampal expression pattern of tdTomato signal in the TRPV1cre X Rosa26tdTomato reporter mouse line. A) IHC shows that in the hippocampus the brightest tdTomato signal is in processes of neurons of the dentate gyrus. B) Representative images of the stratum oriens showing that both the N-T and C-T (Neuromics and Millipore) antibodies recognized a subset of neurons (of $4 \mathrm{~N}$-T positive neurons, only 1 co-localized with the tdTomato signal).

In the hippocampus (Figure $27 \mathrm{~A}$ ), it is important to mention that none of the TRPV1 antibodies seemed to recognize hippocampal regions positive for tdTomato. As mentioned before Figure 9 in the stratum oriens we found a 


\section{RESULTS}

restricted population of neurons that were recognized by all of the TRPV1 antibodies tried in this study (Table 1). In Figure $27 \mathrm{~B}$ it is shown that both the C-T antibody from Neuromics and the N-T antibody from Neuromab, gave a positive immunofluorescence signal in a small population of neurons (likely corresponding to OLM neurons). Nevertheless, we were not able to see any tdTomato fluorescence in those neurons. It is likely that the neurons detected using the C-T antibody from Millipore also correspond to OLM neurons, but we found only one single cell positive for both tdTomato and the C-T antibody (Figure $27 \mathrm{~B}$ ).

In the hypothalamic area and piriform cortex only the C-T antibodies yielded low immunofluorescence in some cells that concomitantly expressed tdTomato (Figure $26 \mathrm{~A}$ and $\mathrm{B})$. In the amygdaloid nucleus we found robust expression of tdTomato, but we were not able to detect TRPV1 fluorescence using any of the three antibodies tested.

\subsubsection{F2 TRPV1-tdTomato transgenic mice show a ubiquitous tdTomato expression pattern}

In order to increase the size of the TRPV1-tdTomato mouse colony we crossed siblings of the F1 generation. Strikingly, when we performed the same immunohistochemical analyses as mentioned in the previous section we found a pattern of increased expression of tdTomato across the brain. The number and intensity of this unexpected ubiquitous tdTomato expression pattern varied between animals of the first generation (F1) and homozygotic F2 animals (Figure 28 A).

As shown in Figure $28 \mathrm{~B}$ we genotyped animals from the F2 generation in order to select homozygotes for $\mathrm{IHC}$ and further breeding. We first noticed that the homozygous mice from the second generation had conspicuously red skin and brain compared to $\mathrm{F} 1$ animals. 
A

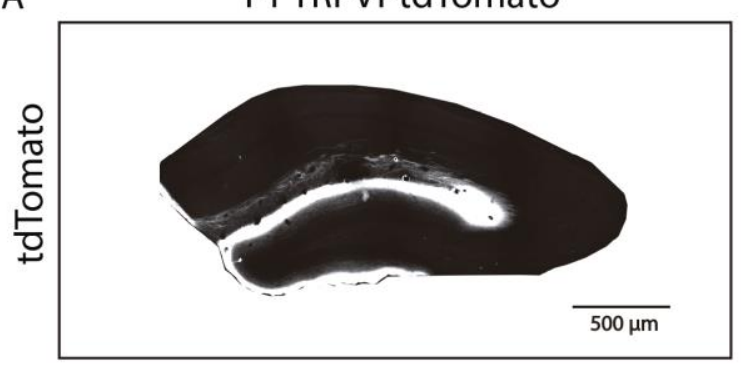

F2 TRPVI-tdTomato (Hom.)
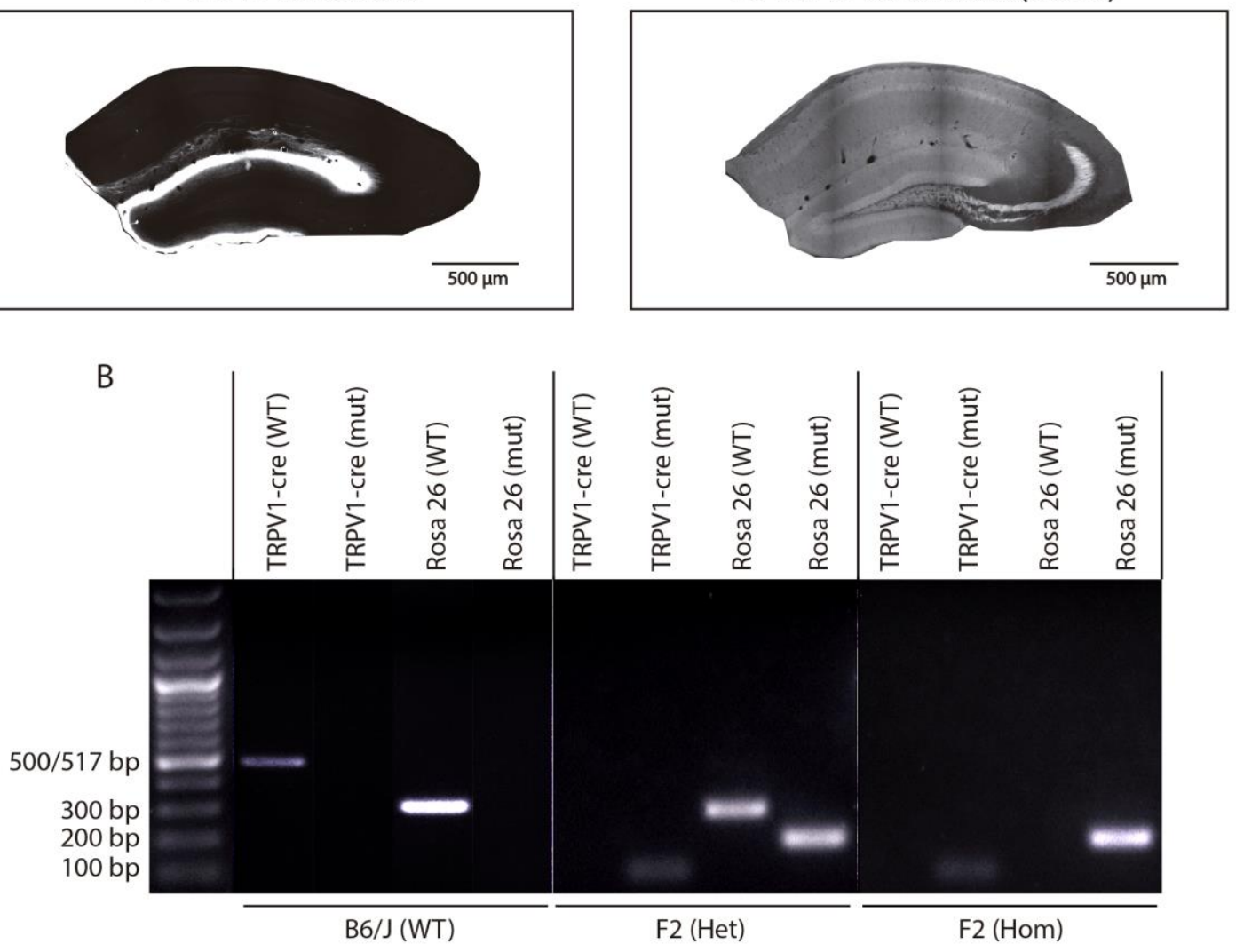

Figure 28. Differences in the tdTomato expression pattern in the hippocampus of the F1 and F2 generation. A) Immunohistochemical comparison of the tdTomato signal in an F1 brain slice compared to a heterozygote and a homozygote from the F2 generation, showing inconsistent expression patterns. B) PCR confirming the genotype of each mouse shown in (A). For each representative image $n=3$ independent genotyping or immunostaining experiments for each antibody were performed.

$\mathrm{IHC}$ in brain slices of animals from the second $\mathrm{F} 2$ generation revealed a completely different expression pattern of tdTomato compared to slices of the F1 generation. We focused our analysis on the hippocampus - the brain structure where most of our research was performed. In the F1 generation we saw a consistent specific small subset of cells expressing tdTomato signal in the brain and hippocampus of offspring of TRPV1-cre male $\mathrm{X}$ tdTomato female and vice versa. Suspiciously, in the homozygote F2 generation, tdTomato fluorescence was extremely high and homogenous, compared to F1 animals. We could not find any homozygotic animal in the F2 generation with the same tdTomato expression pattern as the F1 generation in either the brain or hippocampus. 
RESULTS 
DISCUSSION

\section{DISCUSSION}

\subsection{TRPV1 EXPRESSION IN THE HIPPOCAMPUS}

\subsubsection{TRPV1 and VR.5' sv are differentially expressed in the mouse DRG and hippocampus}

The expression of TRPV1 in the hippocampus has been a subject of intense debate in the last decade. While some groups have found direct and indirect evidence for the expression of TRPV1 in the hippocampus, others have claimed that it is absent in this brain area.

Our results (Figure 4 and Figure 6) confirmed that TRPV1 is expressed in the hippocampus at both mRNA and protein levels, is in accordance with a number of reports that have previously found (using a wide variety of methods) evidence of TRPV1 transcripts and protein expressed in the hippocampus (Bennion et al., 2011; Cavanaugh et al., 2011; Cristino et al., 2006a, 2008; Han et al., 2012; Huang et al., 2014; Merrill et al., 2012; Mezey et al., 2000; Puente et al., 2014; Roberts et al., 2004; Sanchez et al., 2001; Schumacher et al., 2000; Tóth et al., 2005). We used the TRPV1 KO model to test the fidelity of the antibody used for biochemical data and detection of TRPV1 protein expression (Figure 6 A and F). By q-PCR we also validated the deletion of exon 13 that encodes part of the fifth and all of the sixth transmembrane domain, including the putative pore region (Caterina et al., 2000) in the TRPV1 KO model in both DRG and hippocampus (Figure 4).

Consistent with previous results we found that in DRG the mRNA levels for exons encoding the pore region, N-T and C-T exceed the levels detected in the hippocampus (Sanchez et al., 2001; Szallasi et al., 1995) of WT animals (Figure 4 


\section{DISCUSSION}

A). Although compared to the amount TRPV1 mRNA in DRG, the levels detected in the hippocampus appear to be negligible, the modest expression corresponds to reports that the hippocampus is the brain area with the lowest expression of TRPV1 (Han et al., 2012; Menigoz and Boudes, 2011) where it is possibly restricted only to a discrete type of neurons (Cavanaugh et al., 2011).

Our results are also consistent with previous reports showing that the alternative $\mathrm{N}$ T splice isoform VR.5' sv, is expressed in the hippocampus. VR.5' sv is known be present at the same level as TRPV1 in the hippocampus, unlike the proposed ratio of expression of 1:20 (VR.5's sv: TRPV1) in DRG (Sanchez et al., 2001; Schumacher et al., 2000). Similar to these previous reports we found that in DRGs of adult mice the ratio of mRNA of VR.5' sv compared to TRPV1 ( $\mathrm{N}-\mathrm{T}$ and pore reagion) was 1:15 (VR.5' sv: TRPV1) and even 1:44 when compared to TRPV1 C$T$ (Figure $5 \mathrm{~A}$ ). In the hippocampus of adult mice we estimated that the mRNA level of VR.5' sv was actually 10 to 14 times higher than for the TRPV1 pore region and TRPV1 $\mathrm{N}-\mathrm{T}$ respectively (Figure $5 \mathrm{~B}$ ), unlike the 1:1 ratio measured by other groups (Sanchez et al., 2001; Schumacher et al., 2000). Intriguingly, VR.5' sv mRNA expression hippocampus was two times lower than TRPV1 C-T (Figure 5 B). This could be because the primers for C-T detect both VR.5' sv and TRPV1, and hence reflect an aggregate expression of both proteins. A second alternative is that there is another TRPV1 splice variant bearing the C-T, for example TRPV1 $\beta$ which has a mutated N-T but contains the same C-T as full-length TRPV1 (Wang et al., 2004). It would be highly interesting to test if in the hippocampus a different TRPV1 transcriptomic profile exists compared to DRG or other brain areas.

\subsubsection{Quantification of TRPV1 expression during development}

Less is known about the developmental expression of TRPV1 in the CNS during ontogeny. One study showed that TRPV1 is already expressed at E13 in ependymal cells, and its expression increases until E19, when its levels drop (Jo et 


\section{DISCUSSION}

al., 2013). Yet this study only focused on a very specific type of tissue. In postnatal development of mice another study found that TRPV1 expression increased from P21 to 8 weeks after birth (when it reached the highest point) before decreasing, to similar levels as P21, at 16 weeks (Huang et al., 2014). In our study we show for the first time the expression profile of TRPV1 from E13-P21. We show that TRPV1 expression steadily increases during late embryogenesis and postnatal stages persisting at least until P-21 (Figure 6 C).

\subsubsection{Remaining expression of the TRPV1 C-T in TRPV1 KOs}

The pore region of TRPV1 is deleted in the TRPV1 KOs. But in the hippocampus of TRPV1 KO animals we detected a 350-fold increase in mRNA expression of the TRPV1 C-T by Q-PCR (Figure $4 \mathrm{~A}$ and C). We also found that in the hippocampus (but not in DRG) of TRPV1 KO animals the amount of VR.5' sv mRNA tripled that detected in WT samples (Figure $4 \mathrm{~B}$ and $\mathrm{C}$ ). It is possible that the increase in C-T and the VR.5' sV-specific amplicons in TRPV1 KO adult hippocampus represents a compensation of due to the absence of full length TRPV1 receptors (Barbaric et al., 2007; Martins et al., 2014; O'Sullivan et al., 2006) by an increase in the expression of paralogous genes or even alternative splice variants (e.g. VR.5' sv) that could serve as a "backup" through genetic redundancy (Barbaric et al., 2007).

Several cases of mutant mice have shown that aberrant splicing can occur, leading to expression even at protein levels. Our results suggest that either a naturallyoccurring or an aberrant TRPV1-like protein or splice variant possessing the C-T persist in TRPV1 KOs. As shown in Figure $8 \mathrm{~A}$ and $\mathrm{B}$, a subset of hippocampal neurons in vitro that express TRPV1 was still recognized by the C-T antibody, but not by the N-T antibody, in TRPV1 KOs, where the N-T antibody recognizes fulllength TRPV1 by WB (Figure $6 \mathrm{~A}$ and F). Moreover the C-T antibody recognized hippocampal neurons transfected with the VR.5' sv construct (Figure $8 \mathrm{C}$ ), thus (complemented with the Q-PCR results) it is likely that a VR.5' sv-like protein 


\section{DISCUSSION}

remains in the TRPV1 KOs. The C-T antibody also detected a distinct type of inhibitory interneuron (mainly in the oriens lacunosum moleculare layer) in WT and TRPV1 KO hippocampal slices, thus a TRPV1-like remnant or splice isoform likely remains in TRPVI KOs (Figure 9). The remaining fluorescence signal could also be of a homologue of the TRPV1 channel, e.g. TRPV2 and TRPV4, which are 40-50\% homologous to TRPV1 (Liedtke et al., 2000; Park et al., 2011b; Plant and Strotmann, 2007). TRPV2 and TRPV4 partially co-localize with $C$ and $\delta$ fibers of sensory neurons and small-sized DRG neurons, respectively (Caterina et al., 1999; Facer et al., 2007; Ma, 2001). TRPV2 has been reported in multiple brain regions, primarly in those involved in osmoregulation and autonomic responses (Nedungadi et al., 2012), and can form functional hetero-multimers with TRPV1 in neurons of the cerebral cortex (Liapi and Wood, 2005). TRPV4 expression has been observed in pyramidal neurons, some inhibitory neurons and astrocytes of the hippocampus (Shibasaki et al., 2007). However we found that in hippocampal cell cultures, high TRPV1 expressing neurons did not co-localize with TRPV2 or with TRPV4 (Figure $8 \mathrm{D})$, which, interestingly each is present in distinct cell sub-populations.

Unlike the dramatic increase in the C-T and VR.5' sv splice isoform mRNA levels detected in TRPV1 KO animals, we measured a 40-50\% decrease in fluorescence intensity of the C-T antibody in both hippocampal cell cultures and slices from TRPV1 KOs compared to WT (Figure 8 A and B; Figure $9 \mathrm{C}-\mathrm{E}$ ). This likely reflects the fact that the amount of mRNA does not always match the amount of translated protein (Vogel and Marcotte, 2012). For instance, in the case of TRPV1, prolonged exposure to prostaglandin E2 and bradykinin increase TRPV1 and TRPV1b mRNA without changing protein levels in cultured sensory neurons (Mistry et al., 2014). The reduction in C-T protein levels in TRPV1 KOs could indicate the persistence of a TRPV1 isoform. More studies are required to understand the regulation of the abundance and stability of TRPV1 and its isoforms (Barbaric et al., 2007; Schwanhäusser et al., 2011; Vogel and Marcotte, 2012). 


\section{DISCUSSION}

\subsubsection{TRPV1 is expressed functionally in a subset of OLM inhibitory interneurons}

In CA1 pyramidal neurons postsynaptic TRPV1 expression has been observed at both excitatory and inhibitory terminals contacting interneurons of the stratum radiatum, while in CA3 pyramidal neurons pre-synaptic TRPV1 in axons contacting CA1 inhibitory interneurons has been hypothesized to control LTD in these inhibitory interneurons (Cristino et al., 2006b; Gibson et al., 2008; Lee et al., 2015; Micale et al., 2008; Tóth et al., 2005). In the dentate gyrus, TRPV1 was also hypothesized to be post-synaptic at excitatory synapses projecting from the perforant path (Puente et al., 2014) where its activation modulates LTD (Chávez et al., 2010). We found that TRPV1 is both pre- and post-synaptic using a trypsin cleavage assay of synaptosomes from brain homogenates (Figure $6 \mathrm{D}$ and $\mathrm{E}$ ), but our results using this methodology do not specify in which neuronal cell-type or location.

The studies mentioned above detailed a pre- or post-synaptic localization of TRPV1 in excitatory neurons, but not in inhibitory interneurons themselves. TRPV1 was reported to control of plasticity of inhibitory interneurons, but via TRPV1 in excitatory boutons contacting these interneurons (Gibson et al., 2008; Lee et al., 2015).

We detected that high TRPV1-expressing neurons in cultured hippocampal neurons positively correlated with GAD 65 (Figure $10 \mathrm{~A}$ and $\mathrm{B}$ ), in agreement with one previous study suggesting the expression of TRPV1 in GABAergic neurons (Sun et al., 2012). At least 12 different types of inhibitory interneurons are present only in the CA1 region of the hippocampus (Klausberger and Somogyi, 2008; Müller and Remy, 2014). Hippocampal interneurons are usually classified depending on their anatomical location within the hippocampus and the specific protein and peptides they express, such as calretinin, cholecystokinin, neuropeptide $\mathrm{Y}$, calbindin, vasoactive intestinal peptide, parvalbumin and 


\section{DISCUSSION}

somatostatin (Somogyi and Klausberger, 2005). To classify TRPV1-expressing interneurons we tested co-localization of TRPV1 with the markers above and with reelin, which had previously been found to be colocalized with TRPV1-expressing neurons (Cavanaugh et al., 2011). TRPV1 colocalized with $40-50 \%$ of reelin- and somatostatin-positive neurons but not with Parvalbumin 25 (PV 25) or vasointestinal peptide (VIP) positive neurons (Figure $10 \mathrm{~A}$ and $\mathrm{B}$ ). Reelin is an important signaling molecule necessary for the proper formation of cortical and hippocampal laminae during both pre- and post-natal development (Caviness and Rakic, 1978; Stranahan et al., 2013). Since TRPV1 and reelin colocalized, it could be possible that TRPV1 also plays a role in morphogenesis of brain structures. Nevertheless we found no disruptions in the morphology of the brain (Figure 11).

In the developing and adult mouse hippocampus only a few neurons have been found to express TRPV1, which corresponded to reelin-positive neurons. The authors claimed that these cells corresponded to Cajal-Retzius neurons that would eventually disappear from the mature hippocampus (Cavanaugh et al., 2011). Although the number of Cajal-Retzius cells dramatically decreases postnatally, a different population of neurons in the hippocampus, that originate from the medial ganglionic eminence have been identified that also express reelin throughout adulthood (Abraham and Meyer, 2003; Pesold et al., 1998; Ramos-Moreno et al., 2006).

Previous studies have shown that most reelin-expressing neurons do not colocalize with parvalbumin and VIP but do colocalize with somatostatin $(75 \%)$ in the stratum oriens (Alcántara et al., 1998; Miyoshi et al., 2010). We found that neurons co-expressing reelin and somatostatin in the stratum oriens concomitantly had higher levels of TRPV1 (Figure $9 \mathrm{~B}$ ). Furthermore $90 \%$ of these neurons also possess higher pre-synaptic mGluR7 innervation (Figure $9 \mathrm{C}$ ), most likely from excitatory synapses of pyramidal neurons contacting somato-dendrites of TRPV1expressing neurons (Shigemoto et al., 1996, 1997; Tomioka et al., 2014). Together, these data suggest that the high-expressing TRPV1 neurons that we 


\section{DISCUSSION}

identified are oriens lacunosum moleculare (OLM) neurons, which are inhibitory interneurons located in the stratum oriens that are positive for somatostatin and reelin and highly innervated by mGluR7 (Chittajallu et al., 2013; Tomioka et al., 2014). Our observation is supported by the anecdotal report of TRPV1 being expressed in some interneurons in the SO (Cristino et al., 2006b), however this study did not provide more information regarding the identity of these cells.

The presence of TRPV1 in OLM neurons is supported by the compelling observation that OLM neurons (compared to pyramidal neurons) are more susceptible to higher temperatures $\left(40^{\circ} \mathrm{C}-43^{\circ} \mathrm{C}\right)$ experienced during febrile temperatures, what leads to an enhancement of the excitability of OLMsomatostatin neurons and of their firing frequency due to heat (Kim and Connors, 2012). Intriguingly our results showed that reelin is expressed in somatostatinpositive neurons co-expressing TRPV1. Most studies distinguish OLM interneurons as being positive for somatostatin, the most commonly used marker for OLM neurons (Maccaferri et al., 2000; Müller and Remy, 2014). More recent studies suggest that OLM interneurons are not a homogeneous group of inhibitory neurons, but that there are at least 2 types: both express somatostatin and mGluR1 (Chittajallu et al., 2013; Ferraguti et al., 2004; Maccaferri et al., 2000) but one expresses PV25 and originates from the medial ganglionic eminence while another type that originates from the caudal ganglionic eminence expresses reelin and 5-HT ${ }_{3 A} R$ (Chittajallu et al., 2013). Moreover nAChr2 has also been confirmed to be expressed in OLM neurons (but not in all somatostatin-positive neurons) (Leão et al., 2012). Our data suggest that TRPV1-positive OLM cells belong to the subpopulation expressing somatostatin, reelin (and not PV) and nAChr2 which

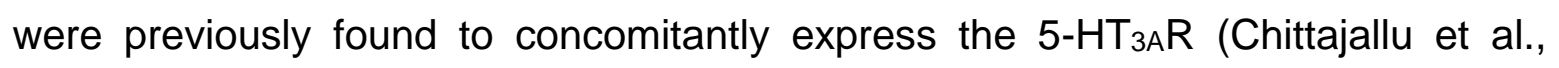
2013). This is supported by the observation that upon increased temperatures $\left(40^{\circ} \mathrm{C}-43^{\circ} \mathrm{C}\right)$ not all OLM became hyper-excitable (Kim et al., 2009). 


\section{DISCUSSION}

\subsubsection{TRPV1-expressing OLM neurons: NGF and BDNF}

We made the novel observation that NGF is highly expressed in TRPV1expressing neurons (Figure $20 \mathrm{~A}$ and $\mathrm{B}$ ). It has previously been reported that NGF is mainly expressed in the cortex and the hippocampus in GABAergic neurons (Biane et al., 2014; Pascual et al., 1998; Rocamora et al., 1996). In the hippocampus, recalling OLM neurons, it is expressed in inhibitory interneurons including somatostatin-expressing interneurons of the stratum oriens that receive septo-hippocampal cholinergic inputs (Acsády et al., 1996; Guo et al., 2012; Leão et al., 2012; Rocamora et al., 1996; Widmer et al., 2006). Additionally in patients with mesial temporal lobe epilepsy TRPV1 and NGF were found to be upregulated in some inhibitory neurons of the cerebral cortex and hippocampus (Sun et al., 2012). It is widely accepted that the binding of NGF to TrKA receptors in sensory neurons leads to downstream signaling cascades driving TRPV1 expression and insertion into the plasma membrane (Anand et al., 2006; Ji et al., 2002; Winston et al., 2001; Zhang et al., 2005). We found that $1 \mathrm{ng} / \mathrm{ml}$ NGF also increased TRPV1 protein levels in hippocampal neuronal cultures (Figure $20 \mathrm{C}-\mathrm{E}$ ), but TRPV1 transfection did not increase NGF expression in the transfected neuron (Figure 20 F). How NGF co-localization correlates with TRPV1 expression in these cells remains unknown. One possibility is that instead of expressing NGF, the TRPV1expressing cells take up NGF that is secreted by contacting cells. Another possibility is that NGF is expressed in TRPV1-expressing cells and as soon as it is released it binds to TrkA or p75 receptors in the same neuron leading to a positive feed-back loop maintaining TRPV1 expression. A problem with this hypothesis is that in the hippocampus the expression of TrkA is controversial; in fact it has been claimed that the only source of TrkA that contact NGF-expressing interneurons from the hippocampus are cholinergic neurons on the medial septum-diagonal band of Broca (Conner et al., 2009; Guo et al., 2012). On the other hand p75 receptor expression appears to be more widespread in the hippocampus, mainly post-synaptically located in CA1 dendritic spines and shafts (Dougherty and Milner, 1999; Woo et al., 2005). If NGF (as mature or as the pro-NGF precursor form) 


\section{DISCUSSION}

expression in inhibitory interneurons with high-TRPV1 levels controls the expression of TRPV1 in these neurons either through interaction with TrKA or p75 remains an open question (Fahnestock et al., 2001; Lewin and Nykjaer, 2014).

We found another neurotrophic factor, BDNF (Figure 21 A-D) was also co-localized with high TRPV1-expressing neurons BDNF, TrKB (the BDNF receptor) and p75 (a receptor for both BDNF and NGF) are expressed throughout different brain regions including the hippocampus (Gray et al., 2013; Hofer et al., 1990; Klein et al., 1990; Lein et al., 2007; Pioro and Cuello, 1990). In the hippocampus, BDNF is highly expressed exclusively in excitatory neurons (e.g. pyramidal and granule cells) of the CA3, CA1 and dentate gyrus in the soma and pre-synaptic terminals of excitatory synapses (Conner et al., 1997; Gray et al., 2013; Yang et al., 2009; Zafra et al., 1990; Zakharenko et al., 2003). If BDNF is post-synaptically expressed and secreted (especially from CA1 pyramidal neurons) to regulate synaptic plasticity is still controversial (Dieni et al., 2012; Hartmann, 2001; Tanaka et al., 2008; Will et al., 2013). We found high somatic (in rat neuronal cultures) (Figure 21 $A$ and $B$ ) and dendritic (in mouse neuronal cultures) (Figure $21 \mathrm{C}$ and D). BDNF levels in high TRPV1-expressing neurons. The difference in the pattern of expression could be caused by the specificity of the antibody, which is higher for mouse tissue. The dendritic (not somatic) pattern of expression seen in murine hippocampal cell cultures agrees more with the acceptance of BDNF not being expressed in inhibitory neurons. However, the dendritic BDNF positive puncta could represent trafficking dense core vesicles transporting BDNF (Dean et al., 2009) that is released onto axons of excitatory neurons contacting TRPV1-positive inhibitory interneurons. In the hippocampus the TrKB receptor is prominently expressed in axons as well as in dendritic shafts and spines of pyramidal and granule cells but is also expressed in inhibitory interneurons including cholinergic neurons of the stratum oriens (Drake et al., 1999; Marty et al., 1996; Spencer et al., 2008; Spencer-Segal et al., 2011; Zachrisson et al., 1996). Interestingly the lowaffinity p75 receptor has also been reported to be expressed in axons of cholinergic neurons. 


\section{DISCUSSION}

\subsubsection{Expression of functional TRPV1 in hippocampal neurons}

Is TRPV1 expressed in OLM-neurons fully functional? In fact, we found that only a small fraction of hippocampal neurons reacted (by influxing $\mathrm{Ca}^{2+}$ ) upon acute (1 $\mu \mathrm{M})$ capsaicin stimulation and as expected none of the TRPVI KO hippocampal neurons responded to capsaicin application (Figure 7). Capsaicin is a specific TRPV1 agonist, known to activate only these receptors (Caterina et al., 1999, 2000). Thus the responses observed in WT (that were absent in TRPV1 KO neurons) must be through TRPV1 activation. Even if the capsaicin-response in these cells was obvious we cannot be completely certain that the neurons undergoing neuronal depolarization detected upon capsaicin during the experiment were actually expressing TRPV1. The observed depolarization could reflect activation of cells contacting the TRPV1 expressing neurons or even a third-party synaptic partner. A previous study using a TRPV1 reporter mouse line failed to detect $\mathrm{Ca}^{2+}$ influx after $10 \mu \mathrm{M}$ capsaicin application in neurons of the dentate gyrus (Cavanaugh et al., 2011). Our study suggests that in mouse hippocampal neurons functional TRPV1 channels exist, which were absent in TRPV1 KO hippocampal neurons. Future studies using calcium imaging of neurons from a SomatostatintdTomato mouse line to identify OLM neurons will verify if the capsaicin-induced calcium responses we observe are specifically due to TRPV1 in OLM cells.

\subsubsection{TRPV1 expression and activation regulate excitatory synaptic innervation in OLM neurons}

Perhaps the most striking discovery in this study was the observation that highTRPV1 expressing neurons were highly innervated by both excitatory (Figure 13) and inhibitory synapses (Figure 14). Our results show that neurons of the stratum oriens with increased TRPV1 expression were concomitantly decorated with a conspicuous abundance of excitatory boutons (Figure 12 A). Early studies 


\section{DISCUSSION}

quantifying synaptic input onto different interneurons identified PV-expressing interneurons of the stratum radiatum to be highly innervated by glutamatergic synapses compared to other neuronal subtypes (Gulyás et al., 1999; Mátyás et al., 2004). To our knowledge the data provided in our study is the first to reveal that high-TRPV1 expressing OLM cells also receive abundant excitatory innervation. One study reported a high co-localization of mGluR7 with vGluT1 (and Elfn1) in excitatory terminals contacting OLM neurons (Tomioka et al., 2014). Furthermore OLM neurons highly express mGluR1 (Ferraguti et al., 2004). OLM neurons are known to be innervated mainly by CA1 excitatory synapses arising from pyramidal neurons (Sun et al., 2014). Dendrites of CA3 OLM neurons have been observed to project to the CA3 stratum radiatum (Gulyás et al., 1993). The main inhibitory synapses made onto OLM neurons originate from presumptive bistratified interneurons (Leão et al., 2012) and even from other OLM neurons (Acsády et al., 1996; Ferraguti et al., 2004; Kogo et al., 2004). There is to date no clear mechanism describing how excitatory and inhibitory pre-synaptic strength to OLM neurons is regulated. Here we describe for the first time that post-synaptic TRPV1 in OLM neurons serves as a synaptogenic factor prompting the formation of excitatory synapses onto these neurons without affecting inhibitory synapse number.

Analysis of high TRPV1-expressing neurons and somatostatin/reelin-positive (OLM) neurons in vitro revealed more excitatory and inhibitory synapses (Figure 13, Figure 14 and Figure 15) on these neurons with greater excitatory and inhibitory presynaptic strength, assayed by higher vGluT1 and VGAT and overlapping syt1u fluorescence intensity than surrounding neurons. TRPV1 has been suggested to affect the dynamics of filopodia and to regulate synaptic vesicle recycling via a localization to synaptic vesicles (Goswami and Hucho, 2007; Goswami et al., 2007, 2010). In our studies the significance of postsynaptic TRPV1 expression for the formation of excitatory synapses was confirmed first by the transfection of heterologous TRPV1 in hippocampal neurons in vitro at DIV2 (analyzed at DIV 12). TRPV1 transfection augmented the strength and number of 


\section{DISCUSSION}

vGluT1-positive synapses onto these neurons and the mEPSC frequency recorded from them (Figure $16 \mathrm{~A}, \mathrm{D}$ and $\mathrm{E}$ ), which reflects an increase in pre-synaptic release probability or number of synapses. Second, with the observation that TRPV1 KO OLM neurons had a tremendous reduction in the number of excitatory vGluT1 synapses formed onto these neurons (Figure $15 \mathrm{~A}$ and $\mathrm{B}$ ).

The functional state of the channel was also important since activation with low (50 $\mathrm{nM})$ or standard $(1 \mu \mathrm{M})$ capsaicin concentration for $21 \mathrm{~h}$ led to an increase in excitatory synapse number in a dose-dependent manner (Figure 13; Figure $15 \mathrm{~A}$ and $B$ ); this findings are, to some extent, similar to previous studies indicating that TRPV1 activation in the first sensory synapse of sensory neurons to DRG, dorsolateral periaqueductal gray neurons and in spinothalamic neurons increased excitatory synaptic input to the depolarized cell (Kim et al., 2009; Sikand and Premkumar, 2007; Xing and Li, 2007). On the other hand, we found that blockade of TRPV1 with $1 \mu \mathrm{M}$ SB-366791 prevented the effects of capsaicin and decreased the number of excitatory synapses (when applied alone) to a similar extent as that observed in TRPV1 KO cultures (Figure $13 \mathrm{~A}-\mathrm{C}$ ). In TRPV1 overexpressing cells SB-366791 decreased the number of excitatory synapses below the number observed on untreated EGFP-expressing neurons (Figure $16 \mathrm{~A}$ and D). Interestingly neither treatment affected vGluT1 or syt1u intensity.

Pre-synaptic TRPV1 activation is known to increase $\mathrm{Ca}^{2+}$, thus prompting glutamate release (without affecting GABAergic synaptic transmission) in sensory neurons, and specific neuronal populations from spinal cord, brainstem, substantia nigra, locus coeruleus, hypothalamus and striatum (Doyle et al., 2002; Marinelli et al., 2002, 2003; Medvedeva et al., 2008; Musella et al., 2009; Sasamura et al., 1998; Yang et al., 1998). Similar to these reports, our results also failed to detect changes in the number and strength of GABAergic boutons contacting high-TRPV1 expressing OLM neurons upon treatment with capsaicin (50 $\mathrm{nM}$ and $1 \mu \mathrm{M}$ ) and 1 $\mu \mathrm{M}$ SB-366791 (Figure $15 \mathrm{C}$ and D; Figure $14 \mathrm{~A}-\mathrm{E}$ ). Surprisingly we found that blockade and knockout of functional TRPV1 caused a significant increase in the 


\section{DISCUSSION}

number of GABA synapses contacting low TRPV1-expressing neurons (Figure 14 $B$ and $C$ ). More difficult to explain are the results obtained from TRPV1-transfected neurons at DIV2 where the overexpression of TRPV1 resulted in decreased GABAergic neurotransmitter release without changing the number of GABAergic inputs or mIPSC amplitude and frequency (Figure 17 A-E) Capsaicin application caused a depression in GABAergic synaptic transmission in the dentate gyrus, through a post-synaptic mechanism without altering GABA release (Chávez et al., 2014). Still the mechanism by which TRPV1 influences inhibitory synaptic transmission remains obscure.

In the hippocampus pre-synaptic TRPV1 activation depresses glutamate release from terminals contacting interneurons in the stratum radiatum (Gibson et al., 2008). Our results on the other hand propose that post-synaptic TRPV1 activation decreases glutamate release onto both high TRPV1 expressing interneurons and other surrounding neurons. An effect of post-synaptic TRPV1 was also reported in the dentate gyrus, but in this case TRPV1 activation decreased synaptic strength by promoting endocytosis of AMPA receptors on the post-synaptic side of the membrane (Chávez et al., 2010). An effect of post-synaptic TRPV1 on pre-synaptic function, similar to our observation was reported in the nucleus accumbens, where post-synaptic TRPV1 activation activated pre-synaptic CB1 to decrease neurotransmitter release (Grueter et al., 2010).

As mentioned in section 5.1.5, high-TRPV1 expressing neurons are decorated with BDNF puncta that are absent in TRPV1 KOs (Figure $21 \mathrm{C}$ and D), suggesting that TRPV1 promotes increases in BDNF. Moreover, TRPV1 transfection at DIV2 increased BDNF in these neurons (Figure $21 \mathrm{E}$ ). BDNF is known to serve both as an anterograde (secreted from the pre- to the post-synapse) and retrograde (postto pre-synaptic) factor that potentiates both excitatory and inhibitory synaptic transmission in the hippocampus upon increased neuronal activity (Bolton et al., 2000; Hartmann, 2001). Thus, we tested if BDNF is necessary for the augmented inhibitory or excitatory pre-synaptic strength observed in high TRPV1-expressing 


\section{DISCUSSION}

interneurons. Blockade of secreted BDNF with $100 \mathrm{ng} / \mathrm{ml}$ TrkB-lgG scavenger did not affect innervation of these neurons or intensity of vGluT1 or vGAT signal. Although blockade at DIV2 slightly increased the number of excitatory synapses on high-expressing TRPV1 neurons, this change was not significant (Figure 25). In addition, the increase in excitatory innervation caused by transfection of TRPV1 at DIV 2 was not prevented with $100 \mathrm{ng} / \mathrm{ml}$ TrKB-IgG treatment at DIV 3. On the contrary, TrkB-lgG treatment slightly increased the number of excitatory terminals on TRPV1-transfected neurons (Figure 24). Together these results rule out BDNF as a candidate molecule that increases innervation of high TRPV1-expressing OLM interneurons.

\subsubsection{Possible TRPV1 endogenous ligands and binding partners in the hippocampus}

What is the endogenous ligand that activates TRPV1 in hippocampal neurons? Since ingested capsaicin does not cross the blood brain barrier and in a normal physiological state $\mathrm{pH}$ and temperature stay constant, these three well-known and TRPV1 agonists are unlikely candidates to trigger TRPV1 channel activation in the hippocampus. However in the PNS as well as in the hippocampus multiple molecules have been proposed to activate TRPV1. In the CNS three endogenous agonists have been proposed to activate TRPV1 in the hippocampus: anandamide, 12-HPETE and N-arachydonoyl-dopamine (NADA) (Ahern, 2003; Al-Hayani et al., 2001; Gibson et al., 2008; Huang et al., 2002). Enzymes responsible for the synthesis of anandamide ( $\mathrm{N}$-acylphosphatidylethanolamine-specific phospholipase D) and 12-HPETE (12-lipoxygenase) (Di Marzo et al., 1994; Hwang et al., 2000) have both been detected in pyramidal neurons of the CA3 region. 12-lipoxygenase has also been detected in CA1 pyramidal neurons as well as in some interneurons in the stratum radiatum (Cristino et al., 2008; Feinmark et al., 2003; Merrill et al., 2012). Induction of LTD in inhibitory interneurons of the stratum radiatum was reported to be dependent on post-synaptic 12-HPETE synthesis triggered by the activation of post-synaptic mGluR1. 12-HPETE then diffused in a retrograde 


\section{DISCUSSION}

fashion to activate pre-synaptic TRPV1 at excitatory synapses leading to a decrease in neurotransmitter release onto the post-synaptic interneuron (Gibson et al., 2008). In another study of excitatory synapses in the dentate gyrus, postsynaptic mGluR5 activation causes anandamide synthesis, which intracellularly activates TRPV1 receptors which cause an internalization of AMPA receptors through the calcineurin / dynamin signaling pathway, thus depressing excitatory synaptic transmission (Chávez et al., 2010). In cortical neurons in vitro NADA has been suggested to cause the recruitment of TRPV1 to filopodia during their elongation, which may affect synaptogenesis (Goswami et al., 2010). Because TRPV1 can be activated by multiple agonists it is difficult to isolate a single endogenous agonist causing an effect on TRPV1. Moreover many agonists of TRPV1 in the hippocampus are not specific only for TRPV1 but also activate other receptors such as the CB1 receptor (Bisogno et al., 2000; Devane et al., 1992; Marzo, 2010), which is activated by anandamide. To date a single unique endogenous TRPV1 agonist has not been isolated in the CNS.

It is not only agonists that mediate the activity of TRPV1 channels. TRPV1 channels can form homo-tetrameric as well as hetero-tetrameric complexes with other TRP channels such as the TRPA1, TRPV2, TRPV3 (Liapi and Wood, 2005; Smith et al., 2002; Staruschenko et al., 2010) and other TRPV1 alternative splice isoforms, such as VR.5' sv (Eilers et al., 2007). VR.5' sv is thought to function as a dominant-negative regulator of TRPV1 activity by reducing its response to capsaicin by $70 \%$ (Eilers et al., 2007). When we transfected VR.5' sv alone (at DIV2) it did not significantly alter the number and strength of VGAT or vGluT1 synapses contacting transfected neurons (Figure $16 \mathrm{~F}-\mathrm{I}$ and Figure $17 \mathrm{~F}-\mathrm{I}$ respectively). On the other hand when TRPV1 was co-transfected at DIV2 with VR.5' sv we found a significant increase in excitatory synapses similar to the levels produced by TRPV1 alone, a significant increase in syt1u at excitatory synapses, and a significant decrease in inhibitory synapse number (Figure $16 \mathrm{~F}-\mathrm{I}$ and Figure 17 F-I). Thus, in terms of synapse formation and strength VR.5' sv appeared to mimic or potentiate the effects of TRPV1. We did not assess whether these effects 


\section{DISCUSSION}

are due to the multimerization of TRPV1 and VR.5' sv. It is still unclear if both isoforms are expressed in OLM neurons. It would be interesting to determine if in certain conditions such as temporal lobe epilepsy VR.5' sv (like TRPV1) is also upregulated to stabilize channel activity.

\subsubsection{Regulation of TRPV1 expression in hippocampal neurons: link to epilepsy}

TRPV1 expression is upregulated in animal models, and in patients, with temporal lobe epilepsy (Anand et al., 2006; Bhaskaran and Smith, 2010; Ji et al., 2002; Sun et al., 2012). NGF is also upregulated during seizure activity (Bengzon et al., 1992; Gall and Isackson, 1989), and NGF induces TRPV1 expression, thus these two molecules may act in the same pathway in epilepsy. One indisputable characteristic of epileptogenesis is hyperexcitability caused by an imbalance in excitation and inhibition which in part is caused by malfunction and death of GABAergic interneurons (Badawy et al., 2009). In the epileptic rat model (induced by pilocarpine injection) TRPV1 activation with $1 \mu \mathrm{M}$ capsaicin was reported to increase basal excitatory synaptic transmission in hippocampal CA1-CA3 pyramidal neurons, while blockade with $10 \mu \mathrm{M}$ capsazepine decreased it (Saffarzadeh et al., 2016). Although in our experiments we did not induce epileptic seizures, we did test the effects of blocking GABAergic inhibitory synaptic transmission with bicuculline, to mimic a malfunction of inhibitory interneurons (Sillito, 1975). As expected, bicuculline application for $21 \mathrm{~h}$ increased excitation and neuronal activity, leading to an increase in BDNF and cFos - two markers of neuronal activity (Morgan and Curran, 1986; Morgan et al., 1987; Zafra et al., 1990, 1992). Interestingly, treatment with bicuculline also significantly increased fluorescence intensity of the C-T TRPV1 antibody in most cells of hippocampal neuronal cultures, most prominently in high-TRPV1 expressing neurons (Figure 23). 


\section{DISCUSSION}

To determine if TRPV1 expression was increased by neuronal activity in general we also tested the effects of forskolin, which increases cAMP levels and increases neuronal activity (Shoback and Brown, 1984). When added either before or after synapse formation in hippocampal cultures, forskolin caused an increase in the fluorescence intensity of the TRPV1 C-T antibody (Figure 22 and Figure 23) in addition to the expected increase of BDNF and cFos levels. There has not been a previous report of forskolin-dependent TRPV1 upregulation, but forskolin blocks TRPV1 desensitization caused by repeated capsaicin application (Schnizler et al., 2008). Intriguingly using WB we failed to detect the bicuculline- or forskolin-induced upregulation of TRPV1 seen with ICC. Because we used the N-T antibody for WB and the C-T antibody for ICC, the discrepancy in results could mean that a splice isoform (e.g VR.5' sv) that is only detected by the C-T antibody is upregulated by these treatments. In the case of bicuculline, only a subset of cells showed increased TRPV1, which may not be detected by WB.

It is still not clear how TRPV1 expression is controlled in the hippocampus. Our results suggest for the first time that NGF upregulates TRPV1 expression in the hippocampus, this is an important observation that together with the changes observed with neuronal activity places TRPV1 at the forefront as a potential indicator or regulator of a misbalance in neuronal activity and excitability that can lead to neuropathies such as status epilepticus.

Interestingly, in patients with temporal lobe epilepsy that exhibit kindling and epileptic seizures and in rodents treated with pilocarpine or kainaic acid (the most common animal models for temporal lobe epilepsy), reorganization of the excitatory hippocampal synaptic network is a key feature of epileptogenesis and development of chronic seizures (Curia et al., 2008; Scheibel et al., 1974). One of the most susceptible regions is the hilus and the mossy fiber pathway. Temporal lobe epilepsy has been correlated with increased sprouting and aberrant innervation of granule neurons, CA3 and CA1 pyramidal neurons but also interneurons of the outer molecular layer and the CA1 region (Babb et al., 1984; 


\section{DISCUSSION}

Kotti et al., 1997; Mathern et al., 1995; Ouardouz et al., 2010; Sanabria et al., 2001). Since NGF and pilocarpine (a known to cholinomimetic agent) induce the aberrant sprouting observed in animals with induced kindling and epilepsy, (whereas the cholinergic antagonist, scopolamine, prevents it) and TrkA receptors co-localize with cholinergic receptors in hippocampal neurons (that receive NGF and cholinergic input from the fimbria and fornix) it has been hypothesized that the actions of NGF in sprouting could potentially be regulated by the cholinergic system (Adams et al., 1997, 2002). Moreover, epileptic episodes increase the number of excitatory inputs and facilitate excitatory synaptic transmission onto somatostatin interneurons of the outer molecular layer as well as interneurons of the stratum oriens and stratum lacunosum moleculare (Mathern et al., 1995, 1998; Ouardouz and Carmant, 2012).

Inhibitory interneurons normally fire rhythmically at theta $(4-12 \mathrm{~Hz})$ or gamma frequencies $(30-80 \mathrm{~Hz})$, to tune the excitability of neuronal networks in a temporal pattern (White et al., 2000). During epileptic pathophysiology spatial and temporal inhibition is disrupted, thus changing the oscillatory activity of the whole system. For instance injection of kainaic acid abruptly diminished the oscillations at theta frequencies, whereas high frequency gamma oscillations are enhanced (Arabadzisz et al., 2005; Dugladze et al., 2007). In animals with persistent epileptic seizures the firing frequency of OLM interneurons in particular shifted their activity from their normal theta frequency (Klausberger and Somogyi, 2008; Müller and Remy, 2014) to gamma oscillations possibly due to an enhancement of the frequency of spontaneous excitatory inputs to these interneurons (Dugladze et al., 2007). Increased firing rates of OLM neurons cause increased gamma oscillations in distal dendrites of hippocampal pyramidal neurons (Tort et al., 2007). Thus hyper-activation of this specific type of interneuron can have profound effects in the total excitatory and inhibitory output of the hippocampal neuronal system. OLM neurons are especially sensitive to febrile temperatures, causing them to become hyper-excitable (Kim and Connors, 2012). This is consistent with our results 


\section{DISCUSSION}

showing that regulation of glutamatergic innervation of OLM neurons expressing TRPV1 is in part driven by the activity of the channel.

In our experiments, capsaicin treatment activated TRPV1 in OLM neurons, which increased the number of excitatory synaptic inputs to these neurons. Because repeated glutamatergic firing of OLM neurons is transformed into oscillations at low frequency, thus inhibiting post-synaptic theta oscillations (Müller and Remy, 2014), TRPV1 expression and signaling could precisely control glutamatergic synapse formation during theta oscillations.

\subsubsection{TRPV1 affects synaptic plasticity in the hippoocampus}

\subsubsection{TRPV1 does not influence LTD in the Schaffer collateral pathway}

TRPV1 activation with $1 \mathrm{mM}$ capsaicin decreases LTD induced by low frequency stimulation ( $3 \mathrm{~Hz})$ ( $\mathrm{Li}$ et al., 2008). However the concentration of capsaicin used in that study exceeds the concentration sufficient to evoke TRPV1-based neuronal depolarization, and may result in desensitization. Using a gradient of more standard concentrations of capsaicin $(1-10 \mu \mathrm{M})$ a later study did not find any effect on LTD induction upon $5 \mathrm{~Hz}$ stimulation in the CA3-CA1 Schaffer collateral pathway (Bennion et al., 2011). However, LTD in TRPV1 KO mice compared to wild-type (whithout the addition of capsaicin) has not been tested. We induced LTD in the Schaffer collateral pathway of wild-type and TRPV1 KO mice with a $1 \mathrm{~Hz}$ low frequency stimulation protocol. We did not find any differences in LTD in TRPV1 KOs compared to wild-type (Figure 19 A). 


\section{DISCUSSION}

\subsubsection{TRPV1 affects LTP in the Schaffer collateral pathway}

TRPV1 KO animals have been reported to have decreased LTP following a 1XTET induction protocol in the Schaffer collateral pathway (Marsch et al., 2007). On the other hand the TRPV1 agonist capsaicin increased LTP in the CA1 region, and this increase was blocked by the TRPV1 specific antagonist SB-366791 (Bennion et al., 2011; Li et al., 2008). Together these were the first observations showing the importance of TRPV1 in normal LTP induction. Later the mechanism was proposed to be mediated through LTD in inhibitory interneurons of the stratum radiatum (Gibson et al., 2008). These authors proposed that activation of mGluR1 receptors in interneurons of the stratum radiatum caused the synthesis of 12-HPETE that acts as a retrograde messenger to activate pre-synaptic TRPV1 channels expressed in excitatory terminals of CA3 pyramidal neurons, which decreases glutamate release to the interneuron in the stratum radiatum of the CA1 region, thereby disinhibiting CA1 pyramidal cells, resulting in an enhancement of LTP (Gibson et al., 2008). Further studies concluded that TRPV1 acts via GABAergic inhibition during LTP since a capsaicin-induced increase in LTP of the Schaffer collateral was abolished in the presence of picrotoxin (a potent GABA-A receptor antagonist) (Bennion et al., 2011), and picrotoxin restored the decreased LTP in TRPV1 KO hippocampi to normal WT levels (Brown et al., 2013).

\subsubsection{Selective activation of OLM neurons rescues the impairment of LTP in TRPV1 KOs}

We performed 1XTET stimulation to induce LTP and confirmed the reduction of LTP in TRPV1 KOs in the Schaffer collateral observed in previous studies (Figure 19 B). Our results indicate that OLM neurons from TRPV1 KO mice have decrease excitatory innervation (most likely from CA1 pyramidal neurons). Such a decrease should be translated into a reduced inhibitory output from the OLM neurons to feedforward inhibitory interneurons of the stratum radiatum, thus intensifying the inhibitory synaptic release of GABA to CA1 pyramidal neurons from the 


\section{DISCUSSION}

interneurons of the stratum radiatum. As mentioned previously, OLM interneurons are the only neurons in the hippocampus that express the nAChr2, thus nicotine specifically activates OLM neurons (Leão et al., 2012; Nakauchi et al., 2007b). We hypothesized that if the reduction in LTP in TRPV1 KOs is due to decreased excitatory innervation of OLM neurons, bypassing this defect by activating OLM neurons with nicotine should rescue the LTP deficits in TRPV1 KOs. To test this, we repeated the LTP experiment in the presence of $5 \mu \mathrm{M}$ nicotine to activate OLM neurons. While nicotine caused a mild increase in LTP in WT hippocampal slices, as previously reported (Nakauchi et al., 2007b), strikingly, nicotine rescued the completely rescued LTP in TRPV1 KOs to wild-type levels (Figure 19 C).

This confirms that TRPV1 is expressed in OLM interneurons and that the LTP deficits observed in TRPV1 knockouts are due to decreased excitatory innervation of OLM neurons. In contrast to the hypothesis provided by Gibson and collaborators (2008) suggesting that LTD in interneurons of the stratum radiatum during LTP (and upon $1 \mu \mathrm{M}$ capsaicin application) results from a decrease in excitatory pre-synaptic strength to straum radiatum interneurons. Alternatively, we infer that the HFS or capsaicin-induced LTD in interneurons of the stratum radiatum could arise from activation of TRPV1 in OLM neurons what would increase the inhibitory input from OLM neurons to interneurons of the stratum radiatum.

In addition, Gibson and collaborators (2008) proposed that activation of presynaptic TRPV1 located in excitatory terminals contacting interneurons of the stratum radiatum caused the observed LTD in these interneurons by depressing excitatory neurotransmitter release. However, most of the literature points out that when TRPV1 is activated in glutamatergic pre-synaptic terminals it rather prompts glutamate release (instead of reducing it) (Jennings et al., 2003; Marinelli et al., 2003; Sasamura et al., 1998; Starowicz et al., 2007). An increase of glutamate release onto inhibitory interneurons would cause them to release GABA and strengthen inhibition (Derbenev et al., 2006; Ferrini et al., 2007; Zhou et al., 2007). 


\section{DISCUSSION}

Gibson and colleagues (2008) found that upon high frequency stimulation mGluR1 activation in stratum radiatum interneurons led to 12-HPETE synthesis. They suggest that 12-HPETE retrogradely diffuses to excitatory synapses (terminating onto interneurons of the stratum radiatum) where it activates TRPV1 and promotes LTD of these interneurons. We did not monitor changes in 12-HPETE synthesis or use it to test TRPV1 activation. Yet the findings of Gibson et al. are highly relevant because 12-HPETE could be a potential candidate that activates TRPV1 in a retrograde fashion from interneurons of the stratum radiatum to OLM neurons, thus controlling a TRPV1-mediated signaling cascade and possibly increasing GABAergic release to the SR which could disinhibit and thereby enhance Schaffer collateral-CA1 LTP.

Our study contributes an essential finding to both the understanding of the function of TRPV1 in the hippocampus, and understanding of the OLM circuit in gating plasticity in the hippocampus. TRPV1 could be an essential part of a mechanism regulating the excitatory input to OLM neurons that is necessary for a correct balance of excitation and inhibition in synaptic plasticity. Some fundamental players have recently been discovered, for example OLM neurons also express the extracellular leucine-rich repeat fibronectin-connecting protein (Elfn1), which recruits mGluR7 receptors to excitatory pre-synaptic terminals of pyramidal neurons contacting OLM cells. The interaction of OLM Elfn1 and mGluR7 regulate excitatory synaptic strength onto OLM interneurons - by decreasing the probability of glutamatergic release - without affecting the number of excitatory synapses on OLM neurons (Müller and Remy, 2014; Sylwestrak and Ghosh, 2012; Tomioka et al., 2014). It will be interesting to further investigate if the mechanism of action of TRPV1 to promote plastic changes in excitatory synaptic strength is associated with the Elfn1-mGluR7 machinery.

Reelin and somatostatin are both expressed in OLM neurons. Secreted somatostatin binds to pre-synaptic somatostatin receptors (putatively somatostatin receptor 2) and decreases both the frequency and amplitude of excitatory (but not 


\section{DISCUSSION}

inhibitory) postsynaptic responses of CA1 pyramidal neurons (Grilli et al., 2004; Tallent and Siggins, 1997). Reelin also affects presynaptic excitatory release probability in CA1 pyramidal neurons, and treatment with reelin enhances LTP induction in the CA1 region (Chen et al., 2005; Hellwig et al., 2011).

CB1 and TRPV1 are both activated by similar agonists to control synaptic transmission (Marzo, 2010). Ablation of CB1 expression in GABAergic interneurons in the hippocampus depressed LTP in the CA3-CA1 pathway and greatly decreased the total dendritic branches and spines in CA1 pyramidal neurons, whereas removal of CB1 from glutamatergic neurons exerted the opposite effect by increasing LTP as well as the density of spines and total branching (Monory et al., 2015). The list of factors and signaling pathways that could, together with TRPV1, coordinate synaptic plasticity is extensive. It remains to be tested, for example, if TRPV1 also gates plasticity in the temporoamonic pathway, and what the endogenous agonist(s) are that regulate the activity of TRPV1 in the hippocampus. OLM neurons are highly sensitive to epileptic and febrile seizures, thus TRPV1 could be an important factor for driving the etiology of neurological disorders such as temporal lobe epilepsy.

\subsection{TRPV1 REPORTER MOUSE LINE}

A relatively new knock in mouse line has been developed using the cre-lox $P$ system to allow specific visualization of cells expressing TRPV1, where a cre recombinase is expressed under the control of the TRPV1 promoter (Cavanaugh et al., 2011). We crossed this mouse line with a mouse line carrying a mutation at the Rosa26 locus with an insertion of a loxP cassette flanking a stop codon preventing the expression of the tdTomato fluorescent reporter gene (Madisen et al., 2010b). When cre is expressed the stop codon is removed thus allowing the expression of the tdTomato in cells where the TRPV1 promoter is expressed. 


\section{DISCUSSION}

Similar to the observations obtained in the original study we saw highly confined expression of the tdTomato fluorescence signal throughout the brain of adult mice of the F1 generation. Following the results of Cavanaugh and collaborators (2011) we confirmed expression in the hypothalamic area (Figure $26 \mathrm{~B}$ ) and few sparse neurons in the stratum oriens of the hippocampus (Figure $27 \mathrm{~B}$ ). In addition, we found a strong tdTomato-positive putative axonal arborisation in the outer molecular layer of the hippocampus (Figure $27 \mathrm{~A}$ ), which was not shown in the original study. Our finding using antibodies that TRPV1 is expressed in OLM neurons of the stratum oriens could not be confirmed using this reporter mouse line because except for one neuron, none of the apparent OLM neurons recognized by antibodies used for IHC colocalized with tdTomato (Figure $27 \mathrm{~B}$ ).

Unexpectedly we found that the expression pattern of tdTomato of the homozygotic F2 generation had a mosaic expression of tdTomato, where fluorescence was observed nonspecifically and ubiquitously throughout the brain. A similar promiscuous expression using cre loxP recombination has been observed for different lines, however the undesired leakage of cre expression occurred only in the second generation, thus siblings of the first generation should be unaffected (Heffner et al., 2012; Weng et al., 2008).

According to these reports the first generation should provide reliable tdTomato expression only in TRPV1 expressing neurons. Nonetheless in our experiments using the $\mathrm{F} 1$ generation we found that tdTomato expression in the hippocampus did not correspond to that detected using commercial antibodies. One possibility is that the antibodies we were using were non-specific, which is known to be a common problem in the field (de Matos et al., 2010; Ward and Rehg, 2014; Yaziji and Barry, 2006).

At first glance it appears that antibodies used in our study might identify a different protein other than TRPV1, because tdTomato-positive cells were not detected by TRPV1 antibodies, and because we found TRPV1-positive immunostained 


\section{DISCUSSION}

neurons in $\mathrm{KO}$ animals. However, we found that the remaining signal is likely a splice variant or aberrant translation of TRPV1 in TRPV1 KO animals. In addition, we confirmed functionally that the neurons detected by TRPV1 antibodies (OLM neurons) do in fact express TRPV1 since we could rescue deficits in LTP in TRPV1 KO hippocampi by selectively activating these neurons with $5 \mu \mathrm{M}$ nicotine.

It is not completely unreasonable to predict that the expression of tdTomato in the TRPV1-tdTomato reporter line even in the first generation is off target. In fact, due to increasing reports of unexpected phenotypes of several cre lines, a Cre strain characterization pipeline at The Jackson Laboratory Cre Repository has been developed. In their webpage, they alert the scientific community to be cautious with the use of cre lines since multiple lines report off-target cells, have variable efficiency of cre loxP recombination, differential inheritance of cre, and toxic cre side-effects among other complications. Finally the developers of the TRPV1-cre line could not rule out the possibility that the expression pattern in the F1 generation could reflect a transient expression during early development (Cavanaugh et al., 2011). Although this could help to trace the fate of TRPV1expressing neurons, it would provide an inaccurate representation of the adultexpression pattern. Since TRPV1 expression in OLM neurons seems to be lower than in other brain areas, the detection threshold may not be enough to allow visualization of cre-loxP recombination. 


\section{CONCLUDING REMARKS}

We found that in the stratum oriens, TRPV1 (and possibly VR.5' SV) is highly expressed in a subset of inhibitory interneurons classified as OLM neurons where it promotes high innervation by glutamatergic synaptic terminals, possibly from pyramidal neurons of the CA1 region of the hippocampus. Deletion of functional TRPV1 dramatically reduced the excitatory innervation on OLM neurons expressing the nACh2 receptor, which ultimately led to a decrease in LTP in the SC pathway of the hippocampus. LTP in TRPV1 KOs was rescued by $5 \mu \mathrm{M}$ nicotine-induced activation of nACh2 receptors on OLM cells possibly by increasing dis-inhibition of the SC-CA1 pathway through a still unknown inhibitory intermediate.

The present study elucidates the role of TRPV1 expression in the hippocampus, the cellular and subcellular localization of functional TRPV1 channels and its role in modulating synaptic strength. Furthermore we demonstrate that TRPV1 plays a role in glutamatergic synaptogenesis on OLM neurons and in the effect of these neurons on LTP induction in the SC in the hippocampus.

Our results also propose different ways in which TRPV1 expression is upregulated in hippocampal neurons. Our results indicate that as in the PNS, NGF is also capable of causing an increase in TRPV1 expression in hippocampal neurons. We also report that neuronal activity increases TRPV1 levels in hippocampal neurons.

We have also found that transfection and increased endogenous expression of TRPV1 (likely restricted to OLM neurons) correlates with high BDNF expression. TRPV1 channel activation was necessary for changes in BDNF upon TRPV1 heterologous expression, since blockade of the channel led to a restoration in BDNF fluorescent intensity in TRPV1-expressing neurons to levels similar to 


\section{CONCLUDING REMARKS}

surrounding neurons. We found that changes in presynaptic strength (syt1u) of excitatory terminals contacting TRPV1 transfected neurons at DIV2 are plausibly controlled by postsynaptic BDNF release. However both the pre-synaptic strength and abundant innervation of glutamatergic synapses on OLM neurons (with high endogenous TRPV1 expression) appear not be caused by BDNF. The induction of more glutamate release onto TRPV1 transfected neurons could be due to a retrograde secretion of BDNF, whereas in OLM neurons another mechanism could be responsible.

Additionally, our results show that the TRPV1-cre reporter mouse line generated by Cavanaugh and collaborators might have some inconsistencies. Although we were able to see expression of the reporter gene (tdTomato) conditionally expressed upon cre activity in some of the brain regions that the original designers of the line reported in the original publication, the immunofluorescence signal detected for three commercial antibodies against TRPV1 only partially co-localized with the tdTomato signal in analyzed areas. In the hippocampus we only saw one cell that was positive for both tdTomato and immune-histochemical fluorescence, but the tdTomato fluorescence in other cells did not correspond to the signal provided by IHC using TRPV1 antibodies.

Prior to the generation of the TRPV1-cre reporter mouse line a series of publications suggested that in the hippocampus the expression and functionality of TRPV1 existed. Cavanaugh et al., 2011 disagreed with these reports citing a highly-restricted expression throughout the hippocampus using the TRPV1 reporter mouse. We discovered that the TRPV1-cre mouse line might not be an accurate reporter of TRPV1 expression. Given our results conclusions formulated using this model should be compared with other methodologies in order to avoid misinterpretation and false results. 


\section{BIBLIOGRAPHY}

Abe, T., and Fujimori, T. (2013). Reporter Mouse Lines for Fluorescence Imaging. Dev. Growth Differ. 55, 390-405.

Abdel-Salam OME (ed.) (2014). Capsaicin as a Therapeutic Molecule (Basel: Springer Basel).

Abraham, H., and Meyer, G. (2003). Reelin-expressing neurons in the postnatal and adult human hippocampal formation. Hippocampus 13, 715-727.

Abremski, K., and Hoess, R. (1984). Bacteriophage P1 site-specific recombination. Purification and properties of the Cre recombinase protein. J. Biol. Chem. 259, 1509-1514.

Acsády, L., Arabadzisz, D., and Freund, T.F. (1996). Correlated morphological and neurochemical features identify different subsets of vasoactive intestinal polypeptide-immunoreactive interneurons in rat hippocampus. Neuroscience 73 , 299-315.

Adamczyk, P., Miszkiel, J., McCreary, A.C., Filip, M., Papp, M., and Przegaliński, E. (2012). The effects of cannabinoid CB1, CB2 and vanilloid TRPV1 receptor antagonists on cocaine addictive behavior in rats. Brain Res. 1444, 45-54.

Adams, B., Sazgar, M., Osehobo, P., Zee, C.E.E.M.V. der, Diamond, J., Fahnestock, M., and Racine, R.J. (1997). Nerve Growth Factor Accelerates Seizure Development, Enhances Mossy Fiber Sprouting, and Attenuates SeizureInduced Decreases in Neuronal Density in the Kindling Model of Epilepsy. J. Neurosci. 17, 5288-5296.

Adams, B., Vaccarella, L., Fahnestock, M., and Racine, R.J. (2002). The cholinergic system modulates kindling and kindling-induced mossy fiber sprouting. Synap. N. Y. N 44, 132-138.

Aguiar, D.C.T. Ana Luisa B.Guimarães, Francisco S.Moreira, Fabrício A. (2009). Anxiolytic-like effects induced by blockade of transient receptor potential vanilloid type 1 (TRPV1) channels in the medial prefrontal cortex of rats. Psychopharmacology (Berl.) 205, 217-225.

Ahern, G.P. (2003). Activation of TRPV1 by the Satiety Factor Oleoylethanolamide. J. Biol. Chem. 278, 30429-30434.

Ahern, G.P., Wang, X., and Miyares, R.L. (2006). Polyamines Are Potent Ligands for the Capsaicin Receptor TRPV1. J. Biol. Chem. 281, 8991-8995. 
Ahmed, S., Reynolds, B.A., and Weiss, S. (1995). BDNF enhances the differentiation but not the survival of CNS stem cell- derived neuronal precursors. J. Neurosci. 15, 5765-5778.

Ahmed, S., Holt, M., Riedel, D., and Jahn, R. (2013). Small-scale isolation of synaptic vesicles from mammalian brain. Nat. Protoc. 8, 998-1009.

Alcántara, S., Ruiz, M., D’Arcangelo, G., Ezan, F., Lecea, L. de, Curran, T., Sotelo, C., and Soriano, E. (1998). Regional and Cellular Patterns of reelin mRNA Expression in the Forebrain of the Developing and Adult Mouse. J. Neurosci. 18, 7779-7799.

Al-Hayani, A., Wease, K.N., Ross, R.A., Pertwee, R.G., and Davies, S.N. (2001). The endogenous cannabinoid anandamide activates vanilloid receptors in the rat hippocampal slice. Neuropharmacology 41, 1000-1005.

Amadesi, S., Cottrell, G.S., Divino, L., Chapman, K., Grady, E.F., Bautista, F., Karanjia, R., Barajas-Lopez, C., Vanner, S., Vergnolle, N., et al. (2006). Proteaseactivated receptor 2 sensitizes TRPV1 by protein kinase $\mathrm{C} \varepsilon$ - and A-dependent mechanisms in rats and mice. J. Physiol. 575, 555-571.

Amaya, F., Oh-hashi, K., Naruse, Y., lijima, N., Ueda, M., Shimosato, G., Tominaga, M., Tanaka, Y., and Tanaka, M. (2003). Local inflammation increases vanilloid receptor 1 expression within distinct subgroups of DRG neurons. Brain Res. 963, 190-196.

Amaya, F., Shimosato, G., Nagano, M., Ueda, M., Hashimoto, S., Tanaka, Y., Suzuki, H., and Tanaka, M. (2004). NGF and GDNF differentially regulate TRPV1 expression that contributes to development of inflammatory thermal hyperalgesia. Eur. J. Neurosci. 20, 2303-2310.

Anand, U., Otto, W.R., Casula, M.A., Day, N.C., Davis, J.B., Bountra, C., Birch, R., and Anand, P. (2006). The effect of neurotrophic factors on morphology, TRPV1 expression and capsaicin responses of cultured human DRG sensory neurons. Neurosci. Lett. 399, 51-56.

Aneiros, E., Cao, L., Papakosta, M., Stevens, E.B., Phillips, S., and Grimm, C. (2011). The biophysical and molecular basis of TRPV1 proton gating. EMBO J. 30, 994-1002.

Apfel, S.C., Wright, D.E., Wiideman, A.M., Dormia, C., Snider, W.D., and Kessler, J.A. (1996). Nerve Growth Factor Regulates the Expression of Brain-Derived Neurotrophic Factor mRNA in the Peripheral Nervous System. Mol. Cell. Neurosci. 7, 134-142.

Arabadzisz, D., Antal, K., Parpan, F., Emri, Z., and Fritschy, J.-M. (2005). Epileptogenesis and chronic seizures in a mouse model of temporal lobe epilepsy 
are associated with distinct EEG patterns and selective neurochemical alterations in the contralateral hippocampus. Exp. Neurol. 194, 76-90.

Babb, T.L., Brown, W.J., Pretorius, J., Davenport, C., Lieb, J.P., and Crandall, P.H. (1984). Temporal lobe volumetric cell densities in temporal lobe epilepsy. Epilepsia $25,729-740$.

Badawy, R.A.B., Harvey, A.S., and Macdonell, R.A.L. (2009). Cortical hyperexcitability and epileptogenesis: understanding the mechanisms of epilepsy part 1. J. Clin. Neurosci. Off. J. Neurosurg. Soc. Australas. 16, 355-365.

Bailey, C.H., Kandel, E.R., and Harris, K.M. (2015). Structural Components of Synaptic Plasticity and Memory Consolidation. Cold Spring Harb. Perspect. Biol. 7, a021758.

Balordi, F., and Fishell, G. (2007). Mosaic Removal of Hedgehog Signaling in the Adult SVZ Reveals That the Residual Wild-Type Stem Cells Have a Limited Capacity for Self-Renewal. J. Neurosci. 27, 14248-14259.

Barakat-Walter, I. (1996). Brain-derived neurotrophic factor-like immunoreactivity is localized mainly in small sensory neurons of rat dorsal root ganglia. J. Neurosci. Methods 68, 281-288.

Barbaric, I., Miller, G., and Dear, T.N. (2007). Appearances can be deceiving: phenotypes of knockout mice. Brief. Funct. Genomic. Proteomic. 6, 91-103.

Barde, Y.A., Edgar, D., and Thoenen, H. (1982). Purification of a new neurotrophic factor from mammalian brain. EMBO J. 1, 549-553.

Bellocchio, E.E., Reimer, R.J., Fremeau, R.T., and Edwards, R.H. (2000). Uptake of glutamate into synaptic vesicles by an inorganic phosphate transporter. Science 289, 957-960.

Bengzon, J., Söderström, S., Kokaia, Z., Kokaia, M., Ernfors, P., Persson, H., Ebendal, T., and Lindvall, O. (1992). Widespread increase of nerve growth factor protein in the rat forebrain after kindling-induced seizures. Brain Res. 587, 338342.

Benninger, F., Freund, T.F., and Hájos, N. (2008). Control of excitatory synaptic transmission by capsaicin is unaltered in TRPV1 vanilloid receptor knockout mice. Neurochem. Int. 52, 89-94.

Bennion, D., Jensen, T., Walther, C., Hamblin, J., Wallmann, A., Couch, J., Blickenstaff, J., Castle, M., Dean, L., Beckstead, S., et al. (2011). Transient receptor potential vanilloid 1 agonists modulate hippocampal CA1 LTP via the GABAergic system. Neuropharmacology 61, 730-738. 
Bevan, S., and Winter, J. (1995). Nerve growth factor (NGF) differentially regulates the chemosensitivity of adult rat cultured sensory neurons. J. Neurosci. 15, 49184926.

Bevan, S., Quallo, T., and Andersson, D.A. (2014). TRPV1. In Mammalian Transient Receptor Potential (TRP) Cation Channels, B. Nilius, and V. Flockerzi, eds. (Berlin, Heidelberg: Springer Berlin Heidelberg), pp. 207-245.

Bezaire, M.J., and Soltesz, I. (2013). Quantitative Assessment of CA1 Local Circuits: Knowledge Base for Interneuron-Pyramidal Cell Connectivity. Hippocampus 23, 751-785.

Bhaskaran, M.D., and Smith, B.N. (2010). Effects of TRPV1 activation on synaptic excitation in the dentate gyrus of a mouse model of temporal lobe epilepsy. Exp. Neurol. 223, 529-536.

Bhave, G., Zhu, W., Wang, H., Brasier, D.J., Oxford, G.S., and Gereau, R.W. (2002). cAMP-Dependent Protein Kinase Regulates Desensitization of the Capsaicin Receptor (VR1) by Direct Phosphorylation. Neuron 35, 721-731.

Bhave, G., Hu, H.-J., Glauner, K.S., Zhu, W., Wang, H., Brasier, D.J., Oxford, G.S., and Gereau, R.W. (2003). Protein kinase C phosphorylation sensitizes but does not activate the capsaicin receptor transient receptor potential vanilloid 1 (TRPV1). Proc. Natl. Acad. Sci. 100, 12480-12485.

Biane, J., Conner, J.M., and Tuszynski, M.H. (2014). Nerve growth factor is primarily produced by GABAergic neurons of the adult rat cortex. Front. Cell. Neurosci. 8.

Birnbaumer, L., Yidirim, E., and Abramowitz, J. (2003). A comparison of the genes coding for canonical TRP channels and their $\mathrm{M}, \mathrm{V}$ and $\mathrm{P}$ relatives. Cell Calcium 33, 419-432.

Bisogno, T., Melck, D., Bobrov MYu, Gretskaya, N.M., Bezuglov, V.V., De Petrocellis, L., and Di Marzo, V. (2000). N-acyl-dopamines: novel synthetic CB(1) cannabinoid-receptor ligands and inhibitors of anandamide inactivation with cannabimimetic activity in vitro and in vivo. Biochem. J. 351, 817-824.

Bizon, J.L., Lauterborn, J.C., and Gall, C.M. (1999). Subpopulations of striatal interneurons can be distinguished on the basis of neurotrophic factor expression. J. Comp. Neurol. 408, 283-298.

Black, D.L. (2003). Mechanisms of Alternative Pre-Messenger RNA Splicing. Annu. Rev. Biochem. 72, 291-336.

Blasco-Ibáñez, J.M., and Freund, T.F. (1995). Synaptic input of horizontal interneurons in stratum oriens of the hippocampal CA1 subfield: structural basis of feed-back activation. Eur. J. Neurosci. 7, 2170-2180. 
Blednov, Y.A., and Harris, R.A. (2009). Deletion of vanilloid receptor (TRPV1) in mice alters behavioral effects of ethanol. Neuropharmacology 56, 814-820.

Bliss, T.V., and Collingridge, G.L. (1993). A synaptic model of memory: long-term potentiation in the hippocampus. Nature 361, 31-39.

Bolton, M.M., Pittman, A.J., and Lo, D.C. (2000). Brain-Derived Neurotrophic Factor Differentially Regulates Excitatory and Inhibitory Synaptic Transmission in Hippocampal Cultures. J. Neurosci. 20, 3221-3232.

Bonci, A., Bernardi, G., Grillner, P., and Mercuri, N.B. (2003). The dopaminecontaining neuron: maestro or simple musician in the orchestra of addiction? Trends Pharmacol. Sci. 24, 172-177.

Bonhoeffer, T. (1996). Neurotrophins and activity-dependent development of the neocortex. Curr. Opin. Neurobiol. 6, 119-126.

Bouabe, H., and Okkenhaug, K. (2013). Gene Targeting in Mice: a Review. Methods Mol. Biol. Clifton NJ 1064, 315-336.

Boulanger, L., and Poo, M. (1999). Gating of BDNF-Induced Synaptic Potentiation by cAMP. Science $284,1982-1984$.

Boutilier, J., Ceni, C., Pagdala, P.C., Forgie, A., Neet, K.E., and Barker, P.A. (2008). Proneurotrophins Require Endocytosis and Intracellular Proteolysis to Induce TrkA Activation. J. Biol. Chem. 283, 12709-12716.

Boyken, J., Grønborg, M., Riedel, D., Urlaub, H., Jahn, R., and Chua, J.J.E. (2013). Molecular Profiling of Synaptic Vesicle Docking Sites Reveals Novel Proteins but Few Differences between Glutamatergic and GABAergic Synapses. Neuron 78, 285-297.

Brauchi, S., Orta, G., Salazar, M., Rosenmann, E., and Latorre, R. (2006). A HotSensing Cold Receptor: C-Terminal Domain Determines Thermosensation in Transient Receptor Potential Channels. J. Neurosci. 26, 4835-4840.

Brauchi, S., Orta, G., Mascayano, C., Salazar, M., Raddatz, N., Urbina, H., Rosenmann, E., Gonzalez-Nilo, F., and Latorre, R. (2007). Dissection of the components for PIP2 activation and thermosensation in TRP channels. Proc. Natl. Acad. Sci. 104, 10246-10251.

Brimble, M.J., and Dyball, R.E.J. (1977). Characterization of the responses of oxytocin- and vasopressin-secreting neurones in the supraoptic nucleus to osmotic stimulation. J. Physiol. 271, 253-271.

Brown, R.E., Jarvis, K.L., and Hyland, K.J. (1989). Protein measurement using bicinchoninic acid: elimination of interfering substances. Anal. Biochem. 180, 136139. 
Brown, T.E., Chirila, A.M., Schrank, B.R., and Kauer, J.A. (2013). Loss of interneuron LTD and attenuated pyramidal cell LTP in Trpv1 and Trpv3 KO mice. Hippocampus 23, 662-671.

Buzsáki, G., Horváth, Z., Urioste, R., Hetke, J., and Wise, K. (1992). Highfrequency network oscillation in the hippocampus. Science 256, 1025-1027.

Cajal, S.R.Y. (1894). The Croonian Lecture: La Fine Structure des Centres Nerveux. Proc. R. Soc. Lond. 55, 444-468.

Calejesan, A.A., Kim, S.J., and Zhuo, M. (2000). Descending facilitatory modulation of a behavioral nociceptive response by stimulation in the adult rat anterior cingulate cortex. Eur. J. Pain Lond. Engl. 4, 83-96.

Canduela, M.-J., Mendizabal-Zubiaga, J., Puente, N., Reguero, L., Elezgarai, I., Ramos-Uriarte, A., Gerrikagoitia, I., and Grandes, P. (2015). Visualization by High Resolution Immunoelectron Microscopy of the Transient Receptor Potential Vanilloid-1 at Inhibitory Synapses of the Mouse Dentate Gyrus. PLoS ONE 10, e0119401.

Cao, E., Liao, M., Cheng, Y., and Julius, D. (2013). TRPV1 structures in distinct conformations reveal activation mechanisms. Nature 504, 113-118.

Caterina, M.J. (2007). Transient receptor potential ion channels as participants in thermosensation and thermoregulation. Am. J. Physiol. - Regul. Integr. Comp. Physiol. 292, R64-R76.

Caterina, M.J., Schumacher, M.A., Tominaga, M., Rosen, T.A., Levine, J.D., and Julius, D. (1997). The capsaicin receptor: a heat-activated ion channel in the pain pathway. Nature 389, 816-824.

Caterina, M.J., Rosen, T.A., Tominaga, M., Brake, A.J., and Julius, D. (1999). A capsaicin-receptor homologue with a high threshold for noxious heat. Nature 398, 436-441.

Caterina, M.J., Leffler, A., Malmberg, A.B., Martin, W.J., Trafton, J., Petersen-Zeitz, K.R., Koltzenburg, M., Basbaum, A.I., and Julius, D. (2000). Impaired nociception and pain sensation in mice lacking the capsaicin receptor. Science 288, 306-313.

Cavanaugh, D.J., Chesler, A.T., Jackson, A.C., Sigal, Y.M., Yamanaka, H., Grant, R., O'Donnell, D., Nicoll, R.A., Shah, N.M., Julius, D., et al. (2011). Trpv1 Reporter Mice Reveal Highly Restricted Brain Distribution and Functional Expression in Arteriolar Smooth Muscle Cells. J. Neurosci. 31, 5067-5077.

Caviness, Jr, and Rakic, and P. (1978). Mechanisms of Cortical Development: A View From Mutations in Mice. Annu. Rev. Neurosci. 1, 297-326. 
Cesare, P., and McNaughton, P. (1996). A novel heat-activated current in nociceptive neurons and its sensitization by bradykinin. Proc. Natl. Acad. Sci. 93, 15435-15439.

Chao, M.V. (2003). Neurotrophins and their receptors: A convergence point for many signalling pathways. Nat. Rev. Neurosci. 4, 299-309.

Chapman, E.R. (2002). Synaptotagmin: A Ca2+ sensor that triggers exocytosis? Nat. Rev. Mol. Cell Biol. 3, 498-508.

Chávez, A.E., Chiu, C.Q., and Castillo, P.E. (2010). TRPV1 activation by endogenous anandamide triggers postsynaptic long-term depression in dentate gyrus. Nat. Neurosci. 13, 1511-1518.

Chávez, A.E., Hernández, V.M., Rodenas-Ruano, A., Chan, C.S., and Castillo, P.E. (2014). Compartment-Specific Modulation of GABAergic Synaptic Transmission by TRPV1 Channels in the Dentate Gyrus. J. Neurosci. 34, 1662116629.

Chen, Y., Beffert, U., Ertunc, M., Tang, T.-S., Kavalali, E.T., Bezprozvanny, I., and Herz, J. (2005). Reelin Modulates NMDA Receptor Activity in Cortical Neurons. J. Neurosci. 25, 8209-8216.

Chiara, G.D., and Imperato, A. (1988). Drugs abused by humans preferentially increase synaptic dopamine concentrations in the mesolimbic system of freely moving rats. Proc. Natl. Acad. Sci. 85, 5274-5278.

Chittajallu, R., Craig, M.T., McFarland, A., Yuan, X., Gerfen, S., Tricoire, L., Erkkila, B., Barron, S.C., Lopez, C.M., Liang, B.J., et al. (2013). Dual origins of functionally distinct O-LM interneurons revealed by differential 5-HT3AR expression. Nat. Neurosci. 16, 1598-1607.

Cho, H.-J., Kim, S.-Y., Park, M.-J., Kim, D.-S., Kim, J.-K., and Chu, M.-Y. (1997a). Expression of mRNA for brain-derived neurotrophic factor in the dorsal root ganglion following peripheral inflammation. Brain Res. 749, 358-362.

Cho, H.-J., Kim, J.-K., Zhou, X.-F., and Rush, R.A. (1997b). Increased brainderived neurotrophic factor immunoreactivity in rat dorsal root ganglia and spinal cord following peripheral inflammation. Brain Res. 764, 269-272.

Chu, C., Zavala, K., Fahimi, A., Lee, J., Xue, Q., Eilers, H., and Schumacher, M.A. (2011). Transcription factors Sp1 and Sp4 regulate TRPV1 gene expression in rat sensory neurons. Mol. Pain 7, 44.

Chung, M.-K., Güler, A.D., and Caterina, M.J. (2008). TRPV1 shows dynamic ionic selectivity during agonist stimulation. Nat. Neurosci. 11, 555-564. 
Ciobanu, C., Reid, G., and Babes, A. (2009). Acute and chronic effects of neurotrophic factors BDNF and GDNF on responses mediated by thermo-sensitive TRP channels in cultured rat dorsal root ganglion neurons. Brain Res. 1284, 5467.

Ciura, S., and Bourque, C.W. (2006). Transient Receptor Potential Vanilloid 1 Is Required for Intrinsic Osmoreception in Organum Vasculosum Lamina Terminalis Neurons and for Normal Thirst Responses to Systemic Hyperosmolality. J. Neurosci. 26, 9069-9075.

Clapham, D.E. (2003). TRP channels as cellular sensors. Nature 426, 517-524.

Cohen, S., Levi-Montalcini, R., and Hamburger, V. (1954). A NERVE GROWTHSTIMULATING FACTOR ISOLATED FROM SARCOM AS 37 AND 180*. Proc. Natl. Acad. Sci. U. S. A. 40, 1014-1018.

Conner, J.M., Lauterborn, J.C., Yan, Q., Gall, C.M., and Varon, S. (1997). Distribution of Brain-Derived Neurotrophic Factor (BDNF) Protein and mRNA in the Normal Adult Rat CNS: Evidence for Anterograde Axonal Transport. J. Neurosci. 17, 2295-2313.

Conner, J.M., Franks, K.M., Titterness, A.K., Russell, K., Merrill, D.A., Christie, B.R., Sejnowski, T.J., and Tuszynski, M.H. (2009). NGF Is Essential for Hippocampal Plasticity and Learning. J. Neurosci. 29, 10883-10889.

Cristino, L., de Petrocellis, L., Pryce, G., Baker, D., Guglielmotti, V., and Di Marzo, V. (2006a). Immunohistochemical localization of cannabinoid type 1 and vanilloid transient receptor potential vanilloid type 1 receptors in the mouse brain. Neuroscience 139, 1405-1415.

Cristino, L., de Petrocellis, L., Pryce, G., Baker, D., Guglielmotti, V., and Di Marzo, V. (2006b). Immunohistochemical localization of cannabinoid type 1 and vanilloid transient receptor potential vanilloid type 1 receptors in the mouse brain. Neuroscience 139, 1405-1415.

Cristino, L., Starowicz, K., De Petrocellis, L., Morishita, J., Ueda, N., Guglielmotti, V., and Di Marzo, V. (2008). Immunohistochemical localization of anabolic and catabolic enzymes for anandamide and other putative endovanilloids in the hippocampus and cerebellar cortex of the mouse brain. Neuroscience 151, 955968.

Curia, G., Longo, D., Biagini, G., Jones, R.S.G., and Avoli, M. (2008). The pilocarpine model of temporal lobe epilepsy. J. Neurosci. Methods 172, 143-157.

Davis, J.B., Gray, J., Gunthorpe, M.J., Hatcher, J.P., Davey, P.T., Overend, P., Harries, M.H., Latcham, J., Clapham, C., Atkinson, K., et al. (2000). Vanilloid 
receptor-1 is essential for inflammatory thermal hyperalgesia. Nature $405,183-$ 187.

Dean, C., Liu, H., Dunning, F.M., Chang, P.Y., Jackson, M.B., and Chapman, E.R. (2009). Synaptotagmin-IV Modulates Synaptic Function and LTP by Regulating BDNF Release. Nat. Neurosci. 12, 767-776.

Del Río, J.A., Heimrich, B., Borrell, V., Förster, E., Drakew, A., Alcántara, S., Nakajima, K., Miyata, T., Ogawa, M., Mikoshiba, K., et al. (1997). A role for CajalRetzius cells and reelin in the development of hippocampal connections. Nature $385,70-74$.

Derbenev, A.V., Monroe, M.J., Glatzer, N.R., and Smith, B.N. (2006). VanilloidMediated Heterosynaptic Facilitation of Inhibitory Synaptic Input to Neurons of the Rat Dorsal Motor Nucleus of the Vagus. J. Neurosci. 26, 9666-9672.

Devane, W.A., Hanus, L., Breuer, A., Pertwee, R.G., Stevenson, L.A., Griffin, G., Gibson, D., Mandelbaum, A., Etinger, A., and Mechoulam, R. (1992). Isolation and structure of a brain constituent that binds to the cannabinoid receptor. Science 258, 1946-1949.

Dhaka, A., Uzzell, V., Dubin, A.E., Mathur, J., Petrus, M., Bandell, M., and Patapoutian, A. (2009). TRPV1 Is Activated by Both Acidic and Basic pH. J. Neurosci. 29, 153-158.

Dieni, S., Matsumoto, T., Dekkers, M., Rauskolb, S., Ionescu, M.S., Deogracias, R., Gundelfinger, E.D., Kojima, M., Nestel, S., Frotscher, M., et al. (2012). BDNF and its pro-peptide are stored in presynaptic dense core vesicles in brain neurons. J. Cell Biol. 196, 775-788.

Di Marzo, V., Fontana, A., Cadas, H., Schinelli, S., Cimino, G., Schwartz, J.-C., and Piomelli, D. (1994). Formation and inactivation of endogenous cannabinoid anandamide in central neurons. Nature 372, 686-691.

Docherty, R.J., Yeats, J.C., Bevan, S., and Boddeke, H.W.G.M. (1996). Inhibition of calcineurin inhibits the desensitization of capsaicin-evoked currents in cultured dorsal root ganglion neurones from adult rats. Pflüg. Arch. 431, 828-837.

Dougherty, K.D., and Milner, T.A. (1999). p75NTR immunoreactivity in the rat dentate gyrus is mostly within presynaptic profiles but is also found in some astrocytic and postsynaptic profiles. J. Comp. Neurol. 407, 77-91.

Doyle, M.W., Bailey, T.W., Jin, Y.-H., and Andresen, M.C. (2002). Vanilloid Receptors Presynaptically Modulate Cranial Visceral Afferent Synaptic Transmission in Nucleus Tractus Solitarius. J. Neurosci. 22, 8222-8229. 
Drake, C.T., Milner, T.A., and Patterson, S.L. (1999). Ultrastructural Localization of Full-Length trkB Immunoreactivity in Rat Hippocampus Suggests Multiple Roles in Modulating Activity-Dependent Synaptic Plasticity. J. Neurosci. 19, 8009-8026.

Dray, A., Forbes, C.A., and Burgess, G.M. (1990). Ruthenium red blocks the capsaicin-induced increase in intracellular calcium and activation of membrane currents in sensory neurones as well as the activation of peripheral nociceptors in vitro. Neurosci. Lett. 110, 52-59.

Dreifuss, J.J., Kalnins, I., Kelly, J.S., and Ruf, K.B. (1971). Action potentials and release of neurohypophysial hormones in vitro. J. Physiol. 215, 805-817.

Dugladze, T., Vida, I., Tort, A.B., Gross, A., Otahal, J., Heinemann, U., Kopell, N.J., and Gloveli, T. (2007). Impaired hippocampal rhythmogenesis in a mouse model of mesial temporal lobe epilepsy. Proc. Natl. Acad. Sci. 104, 17530-17535.

Eckardt, D., Theis, M., Döring, B., Speidel, D., Willecke, K., and Ott, T. (2004). Spontaneous ectopic recombination in cell-type-specific Cre mice removes loxPflanked marker cassettes in vivo. Genesis 38, 159-165.

Eilers, H., Lee, S.-Y., Hau, C.W., Logvinova, A., and Schumacher, M.A. (2007). The rat vanilloid receptor splice variant VR.5'sv blocks TRPV1 activation. Neuroreport 18, 969-973.

Erlander, M.G., and Tobin, A.J. (1991). The structural and functional heterogeneity of glutamic acid decarboxylase: A review. Neurochem. Res. 16, 215-226.

Ernsberger, U. (2009). Role of neurotrophin signalling in the differentiation of neurons from dorsal root ganglia and sympathetic ganglia. Cell Tissue Res. 336, 349-384.

Facer, P., Casula, M.A., Smith, G.D., Benham, C.D., Chessell, I.P., Bountra, C., Sinisi, M., Birch, R., and Anand, P. (2007). Differential expression of the capsaicin receptor TRPV1 and related novel receptors TRPV3, TRPV4 and TRPM8 in normal human tissues and changes in traumatic and diabetic neuropathy. BMC Neurol. 7, 11.

Fahnestock, M., Michalski, B., Xu, B., and Coughlin, M.D. (2001). The Precursor Pro-Nerve Growth Factor Is the Predominant Form of Nerve Growth Factor in Brain and Is Increased in Alzheimer's Disease. Mol. Cell. Neurosci. 18, 210-220.

Feinmark, S.J., Begum, R., Tsvetkov, E., Goussakov, I., Funk, C.D., Siegelbaum, S.A., and Bolshakov, V.Y. (2003). 12-Lipoxygenase Metabolites of Arachidonic Acid Mediate Metabotropic Glutamate Receptor-Dependent Long-Term Depression at Hippocampal CA3-CA1 Synapses. J. Neurosci. 23, 11427-11435.

Fernandes, E., Fernandes, M., and Keeble, J. (2012). The functions of TRPA1 and TRPV1: moving away from sensory nerves. Br. J. Pharmacol. 166, 510-521. 
Ferraguti, F., Cobden, P., Pollard, M., Cope, D., Shigemoto, R., Watanabe, M., and Somogyi, P. (2004). Immunolocalization of metabotropic glutamate receptor $1 a$ (mGluR1a) in distinct classes of interneuron in the CA1 region of the rat hippocampus. Hippocampus 14, 193-215.

Ferrini, F., Salio, C., Vergnano, A.M., and Merighi, A. (2007). Vanilloid receptor-1 (TRPV1)-dependent activation of inhibitory neurotransmission in spinal substantia gelatinosa neurons of mouse. Pain 129, 195-209.

Freund, T. f., and Buzsáki, G. (1996). Interneurons of the hippocampus. Hippocampus 6, 347-470.

Fujii, S., Ji, Z., Morita, N., and Sumikawa, K. (1999). Acute and chronic nicotine exposure differentially facilitate the induction of LTP. Brain Res. 846, 137-143.

Fukuoka, T., Kondo, E., Dai, Y., Hashimoto, N., and Noguchi, K. (2001). BrainDerived Neurotrophic Factor Increases in the Uninjured Dorsal Root Ganglion Neurons in Selective Spinal Nerve Ligation Model. J. Neurosci. 21, 4891-4900.

Funakoshi, K., Nakano, M., Atobe, Y., Goris, R.C., Kadota, T., and Yazama, F. (2006). Differential development of TRPV1-expressing sensory nerves in peripheral organs. Cell Tissue Res. 323, 27-41.

Gall, C.M., and Isackson, P.J. (1989). Limbic seizures increase neuronal production of messenger RNA for nerve growth factor. Science 245, 758-761.

Gamazon, E.R., and Stranger, B.E. (2014). Genomics of alternative splicing: evolution, development and pathophysiology. Hum. Genet. 133, 679-687.

García-Sanz, N., Fernández-Carvajal, A., Morenilla-Palao, C., Planells-Cases, R., Fajardo-Sánchez, E., Fernández-Ballester, G., and Ferrer-Montiel, A. (2004). Identification of a Tetramerization Domain in the C Terminus of the Vanilloid Receptor. J. Neurosci. 24, 5307-5314.

García-Sanz, N., Valente, P., Gomis, A., Fernández-Carvajal, A., FernándezBallester, G., Viana, F., Belmonte, C., and Ferrer-Montiel, A. (2007). A Role of the Transient Receptor Potential Domain of Vanilloid Receptor I in Channel Gating. J. Neurosci. 27, 11641-11650.

García-Martínez, C., Morenilla-Palao, C., Planells-Cases, R., Merino, J.M., and Ferrer-Montiel, A. (2000). Identification of an Aspartic Residue in the P-loop of the Vanilloid Receptor That Modulates Pore Properties. J. Biol. Chem. 275, 3255232558.

Gavva, N.R. (2008). Body-temperature maintenance as the predominant function of the vanilloid receptor TRPV1. Trends Pharmacol. Sci. 29, 550-557. 
Gavva, N.R., Klionsky, L., Qu, Y., Shi, L., Tamir, R., Edenson, S., Zhang, T.J., Viswanadhan, V.N., Toth, A., Pearce, L.V., et al. (2004). Molecular Determinants of Vanilloid Sensitivity in TRPV1. J. Biol. Chem. 279, 20283-20295.

Gavva, N.R., Bannon, A.W., Surapaneni, S., Hovland, D.N., Lehto, S.G., Gore, A., Juan, T., Deng, H., Han, B., Klionsky, L., et al. (2007). The Vanilloid Receptor TRPV1 Is Tonically Activated In Vivo and Involved in Body Temperature Regulation. J. Neurosci. 27, 3366-3374.

Gavva, N.R., Treanor, J.J.S., Garami, A., Fang, L., Surapaneni, S., Akrami, A., Alvarez, F., Bak, A., Darling, M., Gore, A., et al. (2008). Pharmacological blockade of the vanilloid receptor TRPV1 elicits marked hyperthermia in humans: Pain 136, 202-210.

Gee, K.R., Brown, K.A., Chen, W.-N.U., Bishop-Stewart, J., Gray, D., and Johnson, I. (2000). Chemical and physiological characterization of fluo-4 Ca2+-indicator dyes. Cell Calcium 27, 97-106.

Gibson, H.E., Edwards, J.G., Page, R.S., Van Hook, M.J., and Kauer, J.A. (2008). TRPV1 channels mediate long-term depression at synapses on hippocampal interneurons. Neuron 57, 746-759.

Gloveli, T., Dugladze, T., Saha, S., Monyer, H., Heinemann, U., Traub, R.D., Whittington, M.A., and Buhl, E.H. (2005). Differential involvement of oriens/pyramidale interneurones in hippocampal network oscillations in vitro. J. Physiol. 562, 131-147.

Goswami, C., and Hucho, T. (2007). TRPV1 expression-dependent initiation and regulation of filopodia. J. Neurochem. 103, 1319-1333.

Goswami, C., Schmidt, H., and Hucho, F. (2007). TRPV1 at nerve endings regulates growth cone morphology and movement through cytoskeleton reorganization. FEBS J. 274, 760-772.

Goswami, C., Rademacher, N., Smalla, K.-H., Kalscheuer, V., Ropers, H.-H., Gundelfinger, E.D., and Hucho, T. (2010). TRPV1 acts as a synaptic protein and regulates vesicle recycling. J Cell Sci 123, 2045-2057.

Gracheva, E.O., Cordero-Morales, J.F., González-Carcacía, J.A., Ingolia, N.T., Manno, C., Aranguren, C.I., Weissman, J.S., and Julius, D. (2011). Ganglionspecific splicing of TRPV1 underlies infrared sensation in vampire bats. Nature 476, 88-91.

Gray, J.D., MILNER, T.A., and MCEWEN, B.S. (2013). DYNAMIC PLASTICITY: THE ROLE OF GLUCOCORTICOIDS, BRAIN-DERIVED NEUROTROPHIC FACTOR AND OTHER TROPHIC FACTORS. Neuroscience 239, 214-227. 
Grilli, M., Raiteri, L., and Pittaluga, A. (2004). Somatostatin inhibits glutamate release from mouse cerebrocortical nerve endings through presynaptic sst2 receptors linked to the adenylyl cyclase-protein kinase A pathway. Neuropharmacology 46, 388-396.

Groth, R., and Aanonsen, L. (2002). Spinal brain-derived neurotrophic factor (BDNF) produces hyperalgesia in normal mice while antisense directed against either BDNF or trkB, prevent inflammation-induced hyperalgesia. Pain 100, 171181.

Grueter, B.A., Brasnjo, G., and Malenka, R.C. (2010). Postsynaptic TRPV1 triggers cell-type specific LTD in the nucleus accumbens. Nat. Neurosci. 13, 1519-1525.

Gu, H., Marth, J.D., Orban, P.C., Mossmann, H., and Rajewsky, K. (1994). Deletion of a DNA polymerase beta gene segment in T cells using cell type-specific gene targeting. Science 265, 103-106.

Gulyás, A.I., Miles, R., Sík, A., Tóth, K., Tamamaki, N., and Freund, T.F. (1993). Hippocampal pyramidal cells excite inhibitory neurons through a single release site. Nature 366, 683-687.

Gulyás, A.I., Megías, M., Emri, Z., and Freund, T.F. (1999). Total Number and Ratio of Excitatory and Inhibitory Synapses Converging onto Single Interneurons of Different Types in the CA1 Area of the Rat Hippocampus. J. Neurosci. 19, 1008210097.

Gunthorpe, M.J., Rami, H.K., Jerman, J.C., Smart, D., Gill, C.H., Soffin, E.M., Luis Hannan, S., Lappin, S.C., Egerton, J., Smith, G.D., et al. (2004). Identification and characterisation of SB-366791, a potent and selective vanilloid receptor (VR1/TRPV1) antagonist. Neuropharmacology 46, 133-149.

Guo, L., Yeh, M.L., Cuzon Carlson, V.C., Johnson-Venkatesh, E.M., and Yeh, H.H. (2012). Nerve growth factor in the hippocamposeptal system: Evidence for activitydependent anterograde delivery and modulation of synaptic activity. J. Neurosci. 32, 7701-7710.

Hakimizadeh, E., Oryan, S., Hajizadeh moghaddam, A., Shamsizadeh, A., and Roohbakhsh, A. (2012). Endocannabinoid System and TRPV1 Receptors in the Dorsal Hippocampus of the Rats Modulate Anxiety-like Behaviors. Iran. J. Basic Med. Sci. 15, 795-802.

Han, P., Korepanova, A.V., Vos, M.H., Moreland, R.B., Chiu, M.L., and Faltynek, C.R. (2012). Quantification of TRPV1 Protein Levels in Rat Tissues to Understand its Physiological Roles. J. Mol. Neurosci. 50, 23-32.

Hartmann, M. (2001). Synaptic secretion of BDNF after high-frequency stimulation of glutamatergic synapses. EMBO J. 20, 5887-5897. 
Hayase, T. (2011). Differential effects of TRPV1 receptor ligands against nicotineinduced depression-like behaviors. BMC Pharmacol. 11, 6.

Hebb, D.O. (1949). The Organization of Behavior: A Neuropsychological Theory (Wiley).

Heffner, C.S., Herbert Pratt, C., Babiuk, R.P., Sharma, Y., Rockwood, S.F., Donahue, L.R., Eppig, J.T., and Murray, S.A. (2012). Supporting conditional mouse mutagenesis with a comprehensive cre characterization resource. Nat. Commun. 3, 1218.

Hellwig, N., Plant, T.D., Janson, W., Schäfer, M., Schultz, G., and Schaefer, M. (2004). TRPV1 Acts as Proton Channel to Induce Acidification in Nociceptive Neurons. J. Biol. Chem. 279, 34553-34561.

Hellwig, N., Albrecht, N., Harteneck, C., Schultz, G., and Schaefer, M. (2005). Homo- and heteromeric assembly of TRPV channel subunits. J Cell Sci 118, 917928.

Hellwig, S., Hack, I., Kowalski, J., Brunne, B., Jarowyj, J., Unger, A., Bock, H.H., Junghans, D., and Frotscher, M. (2011). Role for Reelin in Neurotransmitter Release. J. Neurosci. 31, 2352-2360.

Hjerling-Leffler, J., AIQatari, M., Ernfors, P., and Koltzenburg, M. (2007). Emergence of Functional Sensory Subtypes as Defined by Transient Receptor Potential Channel Expression. J. Neurosci. 27, 2435-2443.

Ho, K.W., Ward, N.J., and Calkins, D.J. (2012). TRPV1: a stress response protein in the central nervous system. Am. J. Neurodegener. Dis. 1, 1-14.

Hoenderop, J.G.J., Vennekens, R., Müller, D., Prenen, J., Droogmans, G., Bindels, R.J.M., and Nilius, B. (2001). Function and expression of the epithelial Ca2+ channel family: comparison of mammalian ECaC1 and 2. J. Physiol. 537, 747-761.

Hoess, R.H., Ziese, M., and Sternberg, N. (1982). P1 site-specific recombination: nucleotide sequence of the recombining sites. Proc. Natl. Acad. Sci. U. S. A. 79, 3398-3402.

Hofer, M., Pagliusi, S.R., Hohn, A., Leibrock, J., and Barde, Y.A. (1990). Regional distribution of brain-derived neurotrophic factor mRNA in the adult mouse brain. EMBO J. 9, 2459-2464.

Holzer, P. (1991). Capsaicin: cellular targets, mechanisms of action, and selectivity for thin sensory neurons. Pharmacol. Rev. 43, 143-201.

Huang, S.M., Bisogno, T., Trevisani, M., Al-Hayani, A., Petrocellis, L.D., Fezza, F., Tognetto, M., Petros, T.J., Krey, J.F., Chu, C.J., et al. (2002). An endogenous 
capsaicin-like substance with high potency at recombinant and native vanilloid VR1 receptors. Proc. Natl. Acad. Sci. 99, 8400-8405.

Huang, W., Min, J., Liu, Y., He, X., and Peng, B. (2014). Expression of TRPV1 in the C57BL/6 mice brain hippocampus and cortex during development: NeuroReport 25, 379-385.

Hudson, L.J., Bevan, S., Wotherspoon, G., Gentry, C., Fox, A., and Winter, J. (2001). VR1 protein expression increases in undamaged DRG neurons after partial nerve injury. Eur. J. Neurosci. 13, 2105-2114.

Hung, L.W., Wang, I.X., Nikaido, K., Liu, P.Q., Ames, G.F., and Kim, S.H. (1998). Crystal structure of the ATP-binding subunit of an ABC transporter. Nature 396, 703-707.

Hwang, D.-F., and Noguchi, T. (2007). Tetrodotoxin poisoning. Adv. Food Nutr. Res. 52, 141-236.

Hwang, S.W., Cho, H., Kwak, J., Lee, S.-Y., Kang, C.-J., Jung, J., Cho, S., Min, K.H., Suh, Y.-G., Kim, D., et al. (2000). Direct activation of capsaicin receptors by products of lipoxygenases: Endogenous capsaicin-like substances. Proc. Natl. Acad. Sci. 97, 6155-6160.

lida, T., Shimizu, I., Nealen, M.L., Campbell, A., and Caterina, M. (2005). Attenuated fever response in mice lacking TRPV1. Neurosci. Lett. 378, 28-33.

Jancsó, G., Kiraly, E., and Jancsó-Gábor, A. (1977). Pharmacologically induced selective degeneration of chemosensitive primary sensory neurones. Nature 270, 741-743.

Jancsó-Gábor, A., Szolcsányi, J., and Jancsó, N. (1970a). Stimulation and desensitization of the hypothalamic heat-sensitive structures by capsaicin in rats. J. Physiol. 208, 449-459.

Jancsó-Gábor, A., Szolcsányi, J., and Jancsó, N. (1970b). Irreversible impairment of thermoregulation induced by capsaicin and similar pungent substances in rats and guinea-pigs. J. Physiol. 206, 495-507.

Jennings, E.A., Vaughan, C.W., Roberts, L.A., and Christie, M.J. (2003). The actions of anandamide on rat superficial medullary dorsal horn neurons in vitro. J. Physiol. 548, 121-129.

Jeske, N.A., Patwardhan, A.M., Gamper, N., Price, T.J., Akopian, A.N., and Hargreaves, K.M. (2006). Cannabinoid WIN 55,212-2 Regulates TRPV1 Phosphorylation in Sensory Neurons. J. Biol. Chem. 281, 32879-32890.

Jeske, N.A., Por, E.D., Belugin, S., Chaudhury, S., Berg, K.A., Akopian, A.N., Henry, M.A., and Gomez, R. (2011). AKAP150 mediates TRPV1 sensitivity to 


\section{BIBLIOGRAPHY}

phosphatidylinositol-4, 5-bisphosphate. J. Neurosci. Off. J. Soc. Neurosci. 31, 8681-8688.

Ji, R.-R., Samad, T.A., Jin, S.-X., Schmoll, R., and Woolf, C.J. (2002). p38 MAPK Activation by NGF in Primary Sensory Neurons after Inflammation Increases TRPV1 Levels and Maintains Heat Hyperalgesia. Neuron 36, 57-68.

Jo, K.D., Lee, K.-S., Lee, W.T., Hur, M.-S., and Kim, H.-J. (2013). Expression of transient receptor potential channels in the ependymal cells of the developing rat brain. Anat. Cell Biol. 46, 68-78.

Johnson, J., Tian, N., Caywood, M.S., Reimer, R.J., Edwards, R.H., and Copenhagen, D.R. (2003). Vesicular Neurotransmitter Transporter Expression in Developing Postnatal Rodent Retina: GABA and Glycine Precede Glutamate. J. Neurosci. 23, 518-529.

Johnston, G.A. (2013). Advantages of an antagonist: bicuculline and other GABA antagonists. Br. J. Pharmacol. 169, 328-336.

Jones, K.R., Fariñas, I., Backus, C., and Reichardt, L.F. (1994). Targeted Disruption of the BDNF Gene Perturbs Brain and Sensory Neuron Development but Not Motor Neuron Development. Cell 76, 989-999.

Jordt, S.-E., and Julius, D. (2002). Molecular Basis for Species-Specific Sensitivity to "Hot" Chili Peppers. Cell 108, 421-430.

Jordt, S.-E., Tominaga, M., and Julius, D. (2000). Acid potentiation of the capsaicin receptor determined by a key extracellular site. Proc. Natl. Acad. Sci. U. S. A. 97, 8134-8139.

Jun-ichi, M., Satoshi, T., Kimi, A., Fumi, T., Akira, T., Kiyoshi, T., and Ken-ichi, Y. (1989). Expression vector system based on the chicken $\beta$-actin promoter directs efficient production of interleukin-5. Gene 79, 269-277.

Kasckow, J.W., Mulchahey, J.J., and Geracioti, T.D.J. (2004). Effects of the vanilloid agonist olvanil and antagonist capsazepine on rat behaviors. Prog. Neuropsychopharmacol. Biol. Psychiatry 28, 291-295.

Katona, I., Acsády, L., and Freund, T.F. (1999). Postsynaptic targets of somatostatin-immunoreactive interneurons in the rat hippocampus. Neuroscience 88, 37-55.

Katz, L.C., and Shatz, C.J. (1996). Synaptic Activity and the Construction of Cortical Circuits. Science 274, 1133-1138.

Kauer, J.A., and Gibson, H.E. (2009). Hot flash: TRPV channels in the brain. Trends Neurosci. 32, 215-224. 
Kedei, N., Szabo, T., Lile, J.D., Treanor, J.J., Olah, Z., ladarola, M.J., and Blumberg, P.M. (2001). Analysis of the Native Quaternary Structure of Vanilloid Receptor 1 ,. J. Biol. Chem. 276, 28613-28619.

Kessler, R.J., and Fanestil, D.D. (1986). Interference by lipids in the determination of protein using bicinchoninic acid. Anal. Biochem. 159, 138-142.

Khan, N., and Smith, M.T. (2015). Neurotrophins and Neuropathic Pain: Role in Pathobiology. Molecules 20, 10657-10688.

Kim, J., and Connors, B. (2012). High temperatures alter physiological properties of pyramidal cells and inhibitory interneurons in hippocampus. Front. Cell. Neurosci. 6, 27.

Kim, A.Y., Tang, Z., Liu, Q., Patel, K.N., Maag, D., Geng, Y., and Dong, X. (2008). Pirt, a Phosphoinositide-Binding Protein, Functions as a Regulatory Subunit of TRPV1. Cell 133, 475-485.

Kim, H., Cui, L., Kim, J., and Kim, S.J. (2009). Transient receptor potential vanilloid type 1 receptor regulates glutamatergic synaptic inputs to the spinothalamic tract neurons of the spinal cord deep dorsal horn. Neuroscience 160, 508-516.

Kim, J.H., Lee, S.-R., Li, L.-H., Park, H.-J., Park, J.-H., Lee, K.Y., Kim, M.-K., Shin, B.A., and Choi, S.-Y. (2011). High Cleavage Efficiency of a 2A Peptide Derived from Porcine Teschovirus-1 in Human Cell Lines, Zebrafish and Mice. PLoS ONE 6, e18556.

Klausberger, T., and Somogyi, P. (2008). Neuronal Diversity and Temporal Dynamics: The Unity of Hippocampal Circuit Operations. Science 321, 53-57.

Klausberger, T., Márton, L.F., Baude, A., Roberts, J.D.B., Magill, P.J., and Somogyi, P. (2004). Spike timing of dendrite-targeting bistratified cells during hippocampal network oscillations in vivo. Nat. Neurosci. 7, 41-47.

Klein, R., Martin-Zanca, D., Barbacid, M., and Parada, L.F. (1990). Expression of the tyrosine kinase receptor gene trkB is confined to the murine embryonic and adult nervous system. Development 109, 845-850.

Kobayashi, Y., and Hensch, T.K. (2013). Germline recombination by conditional gene targeting with Parvalbumin-Cre lines. Front. Neural Circuits 7.

Köfalvi, A., Oliveira, C.R., and Cunha, R.A. (2006). Lack of evidence for functional TRPV1 vanilloid receptors in rat hippocampal nerve terminals. Neurosci. Lett. 403, 151-156.

Kogo, N., Dalezios, Y., Capogna, M., Ferraguti, F., Shigemoto, R., and Somogyi, P. (2004). Depression of GABAergic input to identified hippocampal neurons by 
group III metabotropic glutamate receptors in the rat. Eur. J. Neurosci. 19, 27272740.

Koh, T.W., and Bellen, H.J. (2003). Synaptotagmin I, a Ca2+ sensor for neurotransmitter release. Trends Neurosci. 26, 413-422.

Koplas, P.A., Rosenberg, R.L., and Oxford, G.S. (1997). The Role of Calcium in the Desensitization of Capsaicin Responses in Rat Dorsal Root Ganglion Neurons. J. Neurosci. 17, 3525-3537.

Korsching, S., Auburger, G., Heumann, R., Scott, J., and Thoenen, H. (1985). Levels of Nerve Growth-Factor and Its Messenger-Rna in the Central NervousSystem of the Rat Correlate with Cholinergic Innervation. Embo J. 4, 1389-1393.

Korte, M., Carroll, P., Wolf, E., Brem, G., Thoenen, H., and Bonhoeffer, T. (1995). Hippocampal long-term potentiation is impaired in mice lacking brain-derived neurotrophic factor. Proc. Natl. Acad. Sci. U. S. A. 92, 8856-8860.

Kosik, K.S., and Finch, E.A. (1987). MAP2 and tau segregate into dendritic and axonal domains after the elaboration of morphologically distinct neurites: an immunocytochemical study of cultured rat cerebrum. J. Neurosci. 7, 3142-3153.

Kotti, T., Riekkinen, P.J., and Miettinen, R. (1997). Characterization of target cells for aberrant mossy fiber collaterals in the dentate gyrus of epileptic rat. Exp. Neurol. 146, 323-330.

Kovalchuk, Y., Hanse, E., Kafitz, K.W., and Konnerth, A. (2002). Postsynaptic Induction of BDNF-Mediated Long-Term Potentiation. Science 295, 1729-1734.

Kwak, J., Wang, M.H., Hwang, S.W., Kim, T.-Y., Lee, S.-Y., and Oh, U. (2000). Intracellular ATP Increases Capsaicin-Activated Channel Activity by Interacting with Nucleotide-Binding Domains. J. Neurosci. 20, 8298-8304.

Latorre, R., Brauchi, S., Orta, G., Zaelzer, C., and Vargas, G. (2007). ThermoTRP channels as modular proteins with allosteric gating. Cell Calcium 42, 427-438.

Latorre, R., Zaelzer, C., and Brauchi, S. (2009). Structure-functional intimacies of transient receptor potential channels. Q. Rev. Biophys. 42, 201-246.

Lau, S.-Y., Procko, E., and Gaudet, R. (2012). Distinct properties of Ca2+calmodulin binding to $\mathrm{N}$ - and $\mathrm{C}$-terminal regulatory regions of the TRPV1 channel. J. Gen. Physiol. 140, 541-555.

Leão, R.N., Mikulovic, S., Leão, K.E., Munguba, H., Gezelius, H., Enjin, A., Patra, K., Eriksson, A., Loew, L.M., Tort, A.B., et al. (2012). OLM interneurons differentially modulate CA3 and entorhinal inputs to hippocampal CA1 neurons. Nat. Neurosci. 15, 1524-1530. 
Lee, J.H., Lee, Y., Ryu, H., Kang, D.W., Lee, J., Lazar, J., Pearce, L.V., Pavlyukovets, V.A., Blumberg, P.M., and Choi, S. (2011). Structural insights into transient receptor potential vanilloid type 1 (TRPV1) from homology modeling, flexible docking, and mutational studies. J. Comput. Aided Mol. Des. 25, 317-327.

Lee, R., Kermani, P., Teng, K.K., and Hempstead, B.L. (2001). Regulation of Cell Survival by Secreted Proneurotrophins. Science 294, 1945-1948.

Lee, S.-H., Ledri, M., Tóth, B., Marchionni, I., Henstridge, C.M., Dudok, B., Kenesei, K., Barna, L., Szabó, S.I., Renkecz, T., et al. (2015). Multiple Forms of Endocannabinoid and Endovanilloid Signaling Regulate the Tonic Control of GABA Release. J. Neurosci. 35, 10039-10057.

Lein, E.S., Hawrylycz, M.J., Ao, N., Ayres, M., Bensinger, A., Bernard, A., Boe, A.F., Boguski, M.S., Brockway, K.S., Byrnes, E.J., et al. (2007). Genome-wide atlas of gene expression in the adult mouse brain. Nature 445, 168-176.

Lever, I.J., Bradbury, E.J., Cunningham, J.R., Adelson, D.W., Jones, M.G., McMahon, S.B., Marvizón, J.C.G., and Malcangio, M. (2001). Brain-Derived Neurotrophic Factor Is Released in the Dorsal Horn by Distinctive Patterns of Afferent Fiber Stimulation. J. Neurosci. 21, 4469-4477.

Levi-Montalcini, R., and Hamburger, V. (1951). Selective growth stimulating effects of mouse sarcoma on the sensory and sympathetic nervous system of the chick embryo. J. Exp. Zool. 116, 321-361.

Levine, E.S., Dreyfus, C.F., Black, I.B., and Plummer, M.R. (1995). Brain-derived neurotrophic factor rapidly enhances synaptic transmission in hippocampal neurons via postsynaptic tyrosine kinase receptors. Proc. Natl. Acad. Sci. U. S. A. 92, 8074-8077.

Lewin, G.R. (1996). Neurotrophins and the Specification of Neuronal Phenotype. Philos. Trans. R. Soc. Lond. B Biol. Sci. 351, 405-411.

Lewin, G.R., and Barde, Y.-A. (1996). Physiology of the Neurotrophins. Annu. Rev. Neurosci. 19, 289-317.

Lewin, G.R., and Mendell, L.M. (1994). Regulation of cutaneous C-fiber heat nociceptors by nerve growth factor in the developing rat. J. Neurophysiol. 71, 941949.

Lewin, G.R., and Nykjaer, A. (2014). Pro-neurotrophins, sortilin, and nociception. Eur. J. Neurosci. 39, 363-374.

Li, H.-B., Mao, R.-R., Zhang, J.-C., Yang, Y., Cao, J., and Xu, L. (2008). Antistress Effect of TRPV1 Channel on Synaptic Plasticity and Spatial Memory. Biol. Psychiatry 64, 286-292. 
Li, J., Mahajan, A., and Tsai, M.-D. (2006). Ankyrin Repeat: A Unique Motif Mediating Protein-Protein Interactions†. Biochemistry (Mosc.) 45, 15168-15178.

Liao, M., Cao, E., Julius, D., and Cheng, Y. (2013). Structure of the TRPV1 ion channel determined by electron cryo-microscopy. Nature 504, 107-112.

Liapi, A., and Wood, J.N. (2005). Extensive co-localization and heteromultimer formation of the vanilloid receptor-like protein TRPV2 and the capsaicin receptor TRPV1 in the adult rat cerebral cortex. Eur. J. Neurosci. 22, 825-834.

Liedtke, W., and Kim, C. (2005). Functionality of the TRPV subfamily of TRP ion channels: add mechano-TRP and osmo-TRP to the lexicon! Cell. Mol. Life Sci. CMLS 62, 2985-3001.

Liedtke, W., Choe, Y., Martí-Renom, M.A., Bell, A.M., Denis, C.S., Šali, A., Hudspeth, A.J., Friedman, J.M., and Heller, S. (2000). Vanilloid receptor-related osmotically activated channel (VR-OAC), a candidate vertebrate osmoreceptor. Cell 103, 525-535.

Linden, D.J. (1994). Long-term synaptic depression in the mammalian brain. Neuron 12, 457-472.

Lishko, P.V., Procko, E., Jin, X., Phelps, C.B., and Gaudet, R. (2007). The Ankyrin Repeats of TRPV1 Bind Multiple Ligands and Modulate Channel Sensitivity. Neuron 54, 905-918.

Liu, L., and Simon, S.A. (1996). Capsaicin-induced currents with distinct desensitization and $\mathrm{Ca} 2+$ dependence in rat trigeminal ganglion cells. J. Neurophysiol. 75, 1503-1514.

Lu, B., Pang, P.T., and Woo, N.H. (2005). The yin and yang of neurotrophin action. Nat. Rev. Neurosci. 6, 603-614.

Lu, P., Ewald, A.J., Martin, G.R., and Werb, Z. (2008). Genetic mosaic analysis reveals FGF receptor 2 function in terminal end buds during mammary gland branching morphogenesis. Dev. Biol. 321, 77-87.

Luo, H., Cheng, J., Han, J.-S., and Wan, Y. (2004). Change of vanilloid receptor 1 expression in dorsal root ganglion and spinal dorsal horn during inflammatory nociception induced by complete Freund's adjuvant in rats. Neuroreport 15, 655658.

Luo, W., Wickramasinghe, S.R., Savitt, J.M., Griffin, J.W., Dawson, T.M., and Ginty, D.D. (2007). A Hierarchical NGF Signaling Cascade Controls RetDependent and Ret-Independent Events during Development of Nonpeptidergic DRG Neurons. Neuron 54, 739-754. 
Ma, Q.P. (2001). Vanilloid receptor homologue, VRL1, is expressed by both A- and C-fiber sensory neurons. Neuroreport 12, 3693-3695.

Maccaferri, G., David, J., Roberts, B., Szucs, P., Cottingham, C.A., and Somogyi, P. (2000). Cell surface domain specific postsynaptic currents evoked by identified GABAergic neurones in rat hippocampus in vitro. J. Physiol. 524, 91-116.

Madisen, L., Zwingman, T.A., Sunkin, S.M., Oh, S.W., Zariwala, H.A., Gu, H., Ng, L.L., Palmiter, R.D., Hawrylycz, M.J., Jones, A.R., et al. (2010a). A robust and high-throughput Cre reporting and characterization system for the whole mouse brain. Nat. Neurosci. 13, 133-140.

Madisen, L., Zwingman, T.A., Sunkin, S.M., Oh, S.W., Zariwala, H.A., Gu, H., Ng, L.L., Palmiter, R.D., Hawrylycz, M.J., Jones, A.R., et al. (2010b). A robust and high-throughput Cre reporting and characterization system for the whole mouse brain. Nat. Neurosci. 13, 133-140.

Maeda, H., and Mogenson, G.J. (1982). Effects of peripheral stimulation on the activity of neurons in the ventral tegmental area, substantia nigra and midbrain reticular formation of rats. Brain Res. Bull. 8, 7-14.

Maione, S., De Petrocellis, L., de Novellis, V., Moriello, A.S., Petrosino, S., Palazzo, E., Rossi, F.S., Woodward, D.F., and Di Marzo, V. (2007). Analgesic actions of $\mathrm{N}$-arachidonoyl-serotonin, a fatty acid amide hydrolase inhibitor with antagonistic activity at vanilloid TRPV1 receptors. Br. J. Pharmacol. 150, 766-781.

Maione, S., Starowicz, K., Cristino, L., Guida, F., Palazzo, E., Luongo, L., Rossi, F., Marabese, I., Novellis, V. de, and Marzo, V.D. (2009). Functional Interaction Between TRPV1 and $\mu$-Opioid Receptors in the Descending Antinociceptive Pathway Activates Glutamate Transmission and Induces Analgesia. J. Neurophysiol. 101, 2411-2422.

Malenka, R.C., and Nicoll, and R.A. (1999). Long-Term Potentiation--A Decade of Progress? Science 285, 1870-1874.

Malin, S.A., Molliver, D.C., Koerber, H.R., Cornuet, P., Frye, R., Albers, K.M., and Davis, B.M. (2006). Glial Cell Line-Derived Neurotrophic Factor Family Members Sensitize Nociceptors In Vitro and Produce Thermal Hyperalgesia In Vivo. J. Neurosci. 26, 8588-8599.

Marco, E.M., Echeverry-Alzate, V., López-Moreno, J.A., Giné, E., Peñasco, S., and Viveros, M.P. (2014). Consequences of early life stress on the expression of endocannabinoid-related genes in the rat brain. Behav. Pharmacol. 25, 547-556.

Marinelli, S., Vaughan, C.W., Christie, M.J., and Connor, M. (2002). Capsaicin activation of glutamatergic synaptic transmission in the rat locus coeruleus In vitro. J. Physiol. 543, 531-540. 
Marinelli, S., Marzo, V.D., Berretta, N., Matias, I., Maccarrone, M., Bernardi, G., and Mercuri, N.B. (2003). Presynaptic Facilitation of Glutamatergic Synapses to Dopaminergic Neurons of the Rat Substantia Nigra by Endogenous Stimulation of Vanilloid Receptors. J. Neurosci. 23, 3136-3144.

Marinelli, S., Pascucci, T., Bernardi, G., Puglisi-Allegra, S., and Mercuri, N.B. (2004). Activation of TRPV1 in the VTA Excites Dopaminergic Neurons and Increases Chemical- and Noxious-Induced Dopamine Release in the Nucleus Accumbens. Neuropsychopharmacology 30, 864-870.

Marsch, R., Foeller, E., Rammes, G., Bunck, M., Kössl, M., Holsboer, F., Zieglgänsberger, W., Landgraf, R., Lutz, B., and Wotjak, C.T. (2007). Reduced Anxiety, Conditioned Fear, and Hippocampal Long-Term Potentiation in Transient Receptor Potential Vanilloid Type 1 Receptor-Deficient Mice. J. Neurosci. 27, 832839.

Martins, D., Tavares, I., and Morgado, C. (2014). "Hotheaded": the role OF TRPV1 in brain functions. Neuropharmacology 85, 151-157.

Marty, S., Berninger, B., Carroll, P., and Thoenen, H. (1996). GABAergic Stimulation Regulates the Phenotype of Hippocampal Interneurons through the Regulation of Brain-Derived Neurotrophic Factor. Neuron 16, 565-570.

Marzo, V.D. (2010). Anandamide serves two masters in the brain. Nat. Neurosci. $13,1446-1448$.

Marzo, V.D., and Petrocellis, L.D. (2012). Why do cannabinoid receptors have more than one endogenous ligand? Philos. Trans. R. Soc. Lond. B Biol. Sci. 367, 3216-3228.

Mathern, G.W., Babb, T.L., Pretorius, J.K., and Leite, J.P. (1995). Reactive synaptogenesis and neuron densities for neuropeptide $Y$, somatostatin, and glutamate decarboxylase immunoreactivity in the epileptogenic human fascia dentata. J. Neurosci. 15, 3990-4004.

Mathern, G.W., Pretorius, J.K., Mendoza, D., Lozada, A., Leite, J.P., Chimelli, L., Fried, I., Sakamoto, A.C., Assirati, J.A., and Adelson, P.D. (1998). Increased Hippocampal AMPA and NMDA Receptor Subunit Immunoreactivity in Temporal Lobe Epilepsy Patients. J. Neuropathol. Exp. Neurol. 57, 615-634.

de Matos, L.L., Trufelli, D.C., de Matos, M.G.L., and da Silva Pinhal, M.A. (2010). Immunohistochemistry as an Important Tool in Biomarkers Detection and Clinical Practice. Biomark. Insights 5, 9-20.

Matsuda, N., Lu, H., Fukata, Y., Noritake, J., Gao, H., Mukherjee, S., Nemoto, T., Fukata, M., and Poo, M. (2009). Differential Activity-Dependent Secretion of Brain- 
Derived Neurotrophic Factor from Axon and Dendrite. J. Neurosci. 29, 1418514198.

Matthaei, K.I. (2007). Genetically manipulated mice: a powerful tool with unsuspected caveats. J. Physiol. 582, 481-488.

Mátyás, F., Freund, T.F., and Gulyás, A.I. (2004). Convergence of excitatory and inhibitory inputs onto CCK-containing basket cells in the CA1 area of the rat hippocampus. Eur. J. Neurosci. 19, 1243-1256.

McGaraughty, S., Chu, K.L., Bitner, R.S., Martino, B., Kouhen, R.E., Han, P., Nikkel, A.L., Burgard, E.C., Faltynek, C.R., and Jarvis, M.F. (2003). Capsaicin Infused Into the PAG Affects Rat Tail Flick Responses to Noxious Heat and Alters Neuronal Firing in the RVM. J. Neurophysiol. 90, 2702-2710.

McIntire, S.L., Reimer, R.J., Schuske, K., Edwards, R.H., and Jorgensen, E.M. (1997). Identification and characterization of the vesicular GABA transporter. Nature 389, 870-876.

McMahon, L.L., and Kauer, J.A. (1997). Hippocampal Interneurons Express a Novel Form of Synaptic Plasticity. Neuron 18, 295-305.

Means, A.L., Chytil, A., Moses, H.L., Coffey, R.J., Wright, C.V.E., Taketo, M.M., and Grady, W.M. (2005). Keratin 19 gene drives Cre recombinase expression throughout the early postimplantation mouse embryo. Genesis $42,23-27$.

Medvedeva, Y.V., Kim, M.-S., and Usachev, Y.M. (2008). Mechanisms of Prolonged Presynaptic Ca2+ Signaling and Glutamate Release Induced by TRPV1 Activation in Rat Sensory Neurons. J. Neurosci. Off. J. Soc. Neurosci. 28, 52955311.

Menigoz, A., and Boudes, M. (2011). The Expression Pattern of TRPV1 in Brain. J. Neurosci. 31, 13025-13027.

Merighi, A., Salio, C., Ghirri, A., Lossi, L., Ferrini, F., Betelli, C., and Bardoni, R. (2008). BDNF as a pain modulator. Prog. Neurobiol. 85, 297-317.

Merrill, C.B., McNeil, M., Williamson, R.C., Poole, B.R., Nelson, B., Sudweeks, S., and Edwards, J.G. (2012). Identification of mRNA for endocannabinoid biosynthetic enzymes within hippocampal pyramidal cells and CA1 stratum radiatum interneuron subtypes using quantitative real-time polymerase chain reaction. Neuroscience 218, 89-99.

Mezey, É., Tóth, Z.E., Cortright, D.N., Arzubi, M.K., Krause, J.E., Elde, R., Guo, A., Blumberg, P.M., and Szallasi, A. (2000). Distribution of mRNA for vanilloid receptor subtype 1 (VR1), and VR1-like immunoreactivity, in the central nervous system of the rat and human. Proc. Natl. Acad. Sci. 97, 3655-3660. 
Micale, V., Cristino, L., Tamburella, A., Petrosino, S., Leggio, G.M., Drago, F., and Di Marzo, V. (2008). Anxiolytic Effects in Mice of a Dual Blocker of Fatty Acid Amide Hydrolase and Transient Receptor Potential Vanilloid Type-1 Channels. Neuropsychopharmacology 34, 593-606.

Michael, G.J., Averill, S., Nitkunan, A., Rattray, M., Bennett, D.L.H., Yan, Q., and Priestley, J.V. (1997). Nerve Growth Factor Treatment Increases Brain-Derived Neurotrophic Factor Selectively in TrkA-Expressing Dorsal Root Ganglion Cells and in Their Central Terminations within the Spinal Cord. J. Neurosci. 17, 84768490.

Miranda, A., Nordstrom, E., Mannem, A., Smith, C., Banerjee, B., and Sengupta, J.N. (2007). The Role of TRPV1 in Mechanical and Chemical Visceral Hyperalgesia Following Experimental Colitis. Neuroscience 148, 1021-1032.

Mistry, S., Paule, C.C., Varga, A., Photiou, A., Jenes, A., Avelino, A., Buluwela, L., and Nagy, I. (2014). Prolonged exposure to bradykinin and prostaglandin E2 increases TRPV1 mRNA but does not alter TRPV1 and TRPV1b protein expression in cultured rat primary sensory neurons. Neurosci. Lett. 564, 89-93.

Miyoshi, G., Hjerling-Leffler, J., Karayannis, T., Sousa, V.H., Butt, S.J.B., Battiste, J., Johnson, J.E., Machold, R.P., and Fishell, G. (2010). Genetic fate mapping reveals that the caudal ganglionic eminence produces a large and diverse population of superficial cortical interneurons. J. Neurosci. Off. J. Soc. Neurosci. 30, 1582-1594.

Mohapatra, D.P., and Nau, C. (2003). Desensitization of Capsaicin-activated Currents in the Vanilloid Receptor TRPV1 Is Decreased by the Cyclic AMPdependent Protein Kinase Pathway. J. Biol. Chem. 278, 50080-50090.

Mohapatra, D.P., and Nau, C. (2005). Regulation of Ca2+-dependent Desensitization in the Vanilloid Receptor TRPV1 by Calcineurin and cAMPdependent Protein Kinase. J. Biol. Chem. 280, 13424-13432.

Moiseenkova-Bell, V.Y., Stanciu, L.A., Serysheva, I.I., Tobe, B.J., and Wensel, T.G. (2008). Structure of TRPV1 channel revealed by electron cryomicroscopy. Proc. Natl. Acad. Sci. 105, 7451-7455.

Monory, K., Polack, M., Remus, A., Lutz, B., and Korte, M. (2015). Cannabinoid CB1 Receptor Calibrates Excitatory Synaptic Balance in the Mouse Hippocampus. J. Neurosci. 35, 3842-3850.

Montell, C. (2001). Physiology, Phylogeny, and Functions of the TRP Superfamily of Cation Channels. Sci. Signal. 2001, re1-re1.

Morgan, J.I., and Curran, T. (1986). Role of ion flux in the control of c-fos expression. Nature 322, 552-555. 
Morgan, J.I., Cohen, D.R., Hempstead, J.L., and Curran, T. (1987). Mapping patterns of c-fos expression in the central nervous system after seizure. Science 237, 192-197.

Movahed, P., Jönsson, B.A.G., Birnir, B., Wingstrand, J.A., Jørgensen, T.D., Ermund, A., Sterner, O., Zygmunt, P.M., and Högestätt, E.D. (2005). Endogenous Unsaturated C18 N-Acylethanolamines Are Vanilloid Receptor (TRPV1) Agonists. J. Biol. Chem. 280, 38496-38504.

Müller, C., and Remy, S. (2014). Dendritic inhibition mediated by O-LM and bistratified interneurons in the hippocampus. Front. Synaptic Neurosci. 6.

Mullis, K., Faloona, F., Scharf, S., Saiki, R., Horn, G., and Erlich, H. (1986). Specific enzymatic amplification of DNA in vitro: the polymerase chain reaction. Cold Spring Harb. Symp. Quant. Biol. 51 Pt 1, 263-273.

Musella, A., De Chiara, V., Rossi, S., Prosperetti, C., Bernardi, G., Maccarrone, M., and Centonze, D. (2009). TRPV1 channels facilitate glutamate transmission in the striatum. Mol. Cell. Neurosci. 40, 89-97.

Musella, A., De Chiara, V., Rossi, S., Cavasinni, F., Castelli, M., Cantarella, C., Mataluni, G., Bernardi, G., and Centonze, D. (2010). Transient receptor potential vanilloid 1 channels control acetylcholine/2-arachidonoylglicerol coupling in the striatum. Neuroscience 167, 864-871.

Nabavi, S., Fox, R., Proulx, C.D., Lin, J.Y., Tsien, R.Y., and Malinow, R. (2014). Engineering a memory with LTD and LTP. Nature 511, 348-352.

Naeini, R.S., Witty, M.-F., Séguéla, P., and Bourque, C.W. (2006). An N-terminal variant of Trpv1 channel is required for osmosensory transduction. Nat. Neurosci. 9, 93-98.

Nagy, I., Friston, D., Valente, J.S., Torres Perez, J.V., and Andreou, A.P. (2014). Pharmacology of the capsaicin receptor, transient receptor potential vanilloid type1 ion channel. Prog. Drug Res. Fortschritte Arzneimittelforschung Prog. Rech. Pharm. 68, 39-76.

Nakauchi, S., Brennan, R.J., Boulter, J., and Sumikawa, K. (2007a). Nicotine gates long-term potentiation in the hippocampal CA1 region via the activation of $\alpha 2^{*}$ nicotinic ACh receptors. Eur. J. Neurosci. 25, 2666-2681.

Nakauchi, S., Brennan, R.J., Boulter, J., and Sumikawa, K. (2007b). Nicotine gates long-term potentiation in the hippocampal CA1 region via the activation of $\alpha 2^{*}$ nicotinic ACh receptors. Eur. J. Neurosci. 25, 2666-2681.

Narahashi, T., Moore, J.W., and Scott, W.R. (1964). TETRODOTOXIN BLOCKAGE OF SODIUM CONDUCTANCE INCREASE IN LOBSTER GIANT AXONS. J. Gen. Physiol. 47, 965-974. 
Nedungadi, T.P., Dutta, M., Bathina, C.S., Caterina, M.J., and Cunningham, J.T. (2012). Expression and Distribution of TRPV2 in Rat Brain. Exp. Neurol. 237, 223237.

Nieto-Posadas, A., Picazo-Juárez, G., Llorente, I., Jara-Oseguera, A., MoralesLázaro, S., Escalante-Alcalde, D., Islas, L.D., and Rosenbaum, T. (2012). Lysophosphatidic acid directly activates TRPV1 through a C-terminal binding site. Nat. Chem. Biol. 8, 78-85.

Nilius, B., and Owsianik, G. (2011). The transient receptor potential family of ion channels. Genome Biol. 12, 218.

Nilius, B., and Voets, T. (2005). TRP channels: a TR(I)P through a world of multifunctional cation channels. Pflüg. Arch. 451, 1-10.

Nilius, B., Vennekens, R., Prenen, J., Hoenderop, J.G.J., Droogmans, G., and Bindels, R.J.M. (2001). The Single Pore Residue Asp542 Determines Ca2+ Permeation and Mg2+ Block of the Epithelial Ca2+ Channel. J. Biol. Chem. 276, 1020-1025.

Novakova-Tousova, K., Vyklicky, L., Susankova, K., Benedikt, J., Samad, A., Teisinger, J., and Vlachova, V. (2007). Functional changes in the vanilloid receptor subtype 1 channel during and after acute desensitization. Neuroscience 149, 144 154.

Novellis, V. de, Vita, D., Gatta, L., Luongo, L., Bellini, G., Chiaro, M.D., Marabese, I., Siniscalco, D., Boccella, S., Piscitelli, F., et al. (2011). The blockade of the transient receptor potential vanilloid type 1 and fatty acid amide hydrolase decreases symptoms and central sequelae in the medial prefrontal cortex of neuropathic rats. Mol. Pain 7, 7.

Numazaki, M., Tominaga, T., Toyooka, H., and Tominaga, M. (2002). Direct Phosphorylation of Capsaicin Receptor VR1 by Protein Kinase $C \varepsilon$ and Identification of Two Target Serine Residues. J. Biol. Chem. 277, 13375-13378.

Numazaki, M., Tominaga, T., Takeuchi, K., Murayama, N., Toyooka, H., and Tominaga, M. (2003). Structural determinant of TRPV1 desensitization interacts with calmodulin. Proc. Natl. Acad. Sci. U. S. A. 100, 8002-8006.

Nykjaer, A., Lee, R., Teng, K.K., Jansen, P., Madsen, P., Nielsen, M.S., Jacobsen, C., Kliemannel, M., Schwarz, E., Willnow, T.E., et al. (2004). Sortilin is essential for proNGF-induced neuronal cell death. Nature $427,843-848$.

Obata, K., and Noguchi, K. (2006). BDNF in sensory neurons and chronic pain. Neurosci. Res. 55, 1-10. 
Oh, U., Hwang, S.W., and Kim, D. (1996). Capsaicin activates a nonselective cation channel in cultured neonatal rat dorsal root ganglion neurons. J. Neurosci. $16,1659-1667$.

O'Neil, R.G., and Heller, S. (2005). The mechanosensitive nature of TRPV channels. Pflüg. Arch. 451, 193-203.

O'Neill, J., Senior, T.J., Allen, K., Huxter, J.R., and Csicsvari, J. (2008). Reactivation of experience-dependent cell assembly patterns in the hippocampus. Nat. Neurosci. 11, 209-215.

Orban, P.C., Chui, D., and Marth, J.D. (1992). Tissue- and site-specific DNA recombination in transgenic mice. Proc. Natl. Acad. Sci. U. S. A. 89, 6861-6865.

O'Sullivan, G.J., O'Tuathaigh, C.M., Clifford, J.J., O'Meara, G.F., Croke, D.T., and Waddington, J.L. (2006). Potential and limitations of genetic manipulation in animals. Drug Discov. Today Technol. 3, 173-180.

Ouardouz, M., and Carmant, L. (2012). Changes in inhibitory CA1 network in dual pathology model of epilepsy. Channels $6,18-25$.

Ouardouz, M., Lema, P., Awad, P.N., Di Cristo, G., and Carmant, L. (2010). Nmethyl-D-aspartate, hyperpolarization-activated cation current (Ih) and gammaaminobutyric acid conductances govern the risk of epileptogenesis following febrile seizures in rat hippocampus. Eur. J. Neurosci. 31, 1252-1260.

Palazzo, E., de Novellis, V., Marabese, I., Cuomo, D., Rossi, F., Berrino, L., Rossi, F., and Maione, S. (2002). Interaction between vanilloid and glutamate receptors in the central modulation of nociception. Eur. J. Pharmacol. 439, 69-75.

Pangalos, M., Donoso, J.R., Winterer, J., Zivkovic, A.R., Kempter, R., Maier, N., and Schmitz, D. (2013). Recruitment of oriens-lacunosum-moleculare interneurons during hippocampal ripples. Proc. Natl. Acad. Sci. U. S. A. 110, 4398-4403.

Park, H., and Poo, M. (2013). Neurotrophin regulation of neural circuit development and function. Nat. Rev. Neurosci. 14, 7-23.

Park, C.-K., Xu, Z.-Z., Liu, T., Lü, N., Serhan, C.N., and Ji, R.-R. (2011a). Resolvin D2 Is a Potent Endogenous Inhibitor for Transient Receptor Potential Subtype V1/A1, Inflammatory Pain, and Spinal Cord Synaptic Plasticity in Mice: Distinct Roles of Resolvin D1, D2, and E1. J. Neurosci. 31, 18433-18438.

Park, U., Vastani, N., Guan, Y., Raja, S.N., Koltzenburg, M., and Caterina, M.J. (2011b). TRPV2 KNOCKOUT MICE ARE SUSCEPTIBLE TO PERINATAL LETHALITY BUT DISPLAY NORMAL THERMAL AND MECHANICAL NOCICEPTION. J. Neurosci. Off. J. Soc. Neurosci. 31, 11425-11436. 
Pascual, M., Rocamora, N., Acsády, L., Freund, T.F., and Soriano, E. (1998). Expression of nerve growth factor and neurotrophin-3 mRNAs in hippocampal interneurons: Morphological characterization, levels of expression, and colocalization of nerve growth factor and neurotrophin-3. J. Comp. Neurol. 395, 73-90.

Patapoutian, A. (2005). TRP Channels and Thermosensation. Chem. Senses 30, i193-i194.

Patterson, S.L., Grover, L.M., Schwartzkroin, P.A., and Bothwell, M. (1992). Neurotrophin expression in rat hippocampal slices: A stimulus paradigm inducing LTP in CA1 evokes increases in BDNF and NT-3 mRNAs. Neuron 9, 1081-1088.

Patterson, S.L., Abel, T., Deuel, T.A.S., Martin, K.C., Rose, J.C., and Kandel, E.R. (1996). Recombinant BDNF Rescues Deficits in Basal Synaptic Transmission and Hippocampal LTP in BDNF Knockout Mice. Neuron 16, 1137-1145.

Pavlos, N.J., Grønborg, M., Riedel, D., Chua, J.J.E., Boyken, J., Kloepper, T.H., Urlaub, H., Rizzoli, S.O., and Jahn, R. (2010). Quantitative Analysis of Synaptic Vesicle Rabs Uncovers Distinct Yet Overlapping Roles for Rab3a and Rab27b in Ca2+-Triggered Exocytosis. J. Neurosci. 30, 13441-13453.

Paxinos, G., Franklin, K., B., J. (2012). Paxinos and Franklin's the Mouse Brain in Stereotaxic Coordinates, Fourth Edition (Amsterdam: Academic Press).

Pedersen, S.F., Owsianik, G., and Nilius, B. (2005). TRP channels: An overview. Cell Calcium 38, 233-252.

Pesold, C., Impagnatiello, F., Pisu, M.G., Uzunov, D.P., Costa, E., Guidotti, A., and Caruncho, H.J. (1998). Reelin is preferentially expressed in neurons synthesizing $Y$-aminobutyric acid in cortex and hippocampus of adult rats. Proc. Natl. Acad. Sci. U. S. A. 95, 3221-3226.

Picazo-Juárez, G., Romero-Suárez, S., Nieto-Posadas, A., Llorente, I., JaraOseguera, A., Briggs, M., Mclntosh, T.J., Simon, S.A., Ladrón-de-Guevara, E., Islas, L.D., et al. (2011). Identification of a Binding Motif in the S5 Helix That Confers Cholesterol Sensitivity to the TRPV1 Ion Channel. J. Biol. Chem. 286, 24966-24976.

Pilon, N., Raiwet, D., Viger, R.S., and Silversides, D.W. (2008). Novel pre- and post-gastrulation expression of Gata4 within cells of the inner cell mass and migratory neural crest cells. Dev. Dyn. Off. Publ. Am. Assoc. Anat. 237, 11331143.

Pioro, E.P., and Cuello, A.C. (1990). Distribution of nerve growth factor receptorlike immunoreactivity in the adult rat central nervous system. Effect of colchicine 
and correlation with the cholinergic system-II. Brainstem, cerebellum and spinal cord. Neuroscience 34, 89-110.

Plant, T.D., and Strotmann, R. (2007). TRPV4: A Multifunctional Nonselective Cation Channel with Complex Regulation. In TRP Ion Channel Function in Sensory Transduction and Cellular Signaling Cascades, W.B. Liedtke, and S. Heller, eds. (Boca Raton (FL): CRC Press/Taylor \& Francis),.

Price, T.J., Louria, M.D., Candelario-Soto, D., Dussor, G.O., Jeske, N.A., Patwardhan, A.M., Diogenes, A., Trott, A.A., Hargreaves, K.M., and Flores, C.M. (2005). Treatment of trigeminal ganglion neurons in vitro with NGF, GDNF or BDNF: effects on neuronal survival, neurochemical properties and TRPV1mediated neuropeptide secretion. BMC Neurosci. 6, 4.

Puente, N., Reguero, L., Elezgarai, I., Canduela, M.-J., Mendizabal-Zubiaga, J., Ramos-Uriarte, A., Fernández-Espejo, E., and Grandes, P. (2014). The transient receptor potential vanilloid-1 is localized at excitatory synapses in the mouse dentate gyrus. Brain Struct. Funct. 220, 1187-1194.

Puntambekar, P., Buren, J.V., Raisinghani, M., Premkumar, L.S., and Ramkumar, V. (2004). Direct Interaction of Adenosine with the TRPV1 Channel Protein. J. Neurosci. 24, 3663-3671.

Ramachandran, B., Ahmed, S., Zafar, N., and Dean, C. (2015a). Ethanol inhibits long-term potentiation in hippocampal CA1 neurons, irrespective of lamina and stimulus strength, through neurosteroidogenesis. Hippocampus 25, 106-118.

Ramachandran, B., Ahmed, S., and Dean, C. (2015b). Long-term depression is differentially expressed in distinct lamina of hippocampal CA1 dendrites. Front. Cell. Neurosci. 9.

Ramos-Moreno, T., Galazo, M.J., Porrero, C., Martínez-Cerdeño, V., and Clascá, F. (2006). Extracellular matrix molecules and synaptic plasticity: immunomapping of intracellular and secreted Reelin in the adult rat brain. Eur. J. Neurosci. 23, 401422.

Renteria, R., Jeanes, Z.M., and Morrisett, R.A. (2014). Ethanol attenuation of long term depression in the nucleus accumbens can be overcome by activation of TRPV1 receptors. Alcohol. Clin. Exp. Res. 38, 2763-2769.

Roberts, J.C., Davis, J.B., and Benham, C.D. (2004). [3H]Resiniferatoxin autoradiography in the CNS of wild-type and TRPV1 null mice defines TRPV1 (VR1) protein distribution. Brain Res. 995, 176-183.

Rocamora, N., Pascual, M., Acsàdy, L., Lecea, L. de, Freund, T.F., and Soriano, E. (1996). Expression of NGF and NT3 mRNAs in Hippocampal Interneurons 
Innervated by the GABAergic Septohippocampal Pathway. J. Neurosci. 16, 39914004.

Rotstein, H.G., Pervouchine, D.D., Acker, C.D., Gillies, M.J., White, J.A., Buhl, E.H., Whittington, M.A., and Kopell, N. (2005). Slow and Fast Inhibition and an HCurrent Interact to Create a Theta Rhythm in a Model of CA1 Interneuron Network. J. Neurophysiol. 94, 1509-1518.

Rubino, T., Realini, N., Castiglioni, C., Guidali, C., Viganó, D., Marras, E., Petrosino, S., Perletti, G., Maccarrone, M., Marzo, V.D., et al. (2008). Role in Anxiety Behavior of the Endocannabinoid System in the Prefrontal Cortex. Cereb. Cortex 18, 1292-1301.

Rutter, A.R., Ma, Q.-P., Leveridge, M., and Bonnert, T.P. (2005). Heteromerization and colocalization of TrpV1 and TrpV2 in mammalian cell lines and rat dorsal root ganglia. Neuroreport 16, 1735-1739.

Ryu, S., Liu, B., Yao, J., Fu, Q., and Qin, F. (2007). Uncoupling Proton Activation of Vanilloid Receptor TRPV1. J. Neurosci. 27, 12797-12807.

Saffarzadeh, F., Eslamizade, M.J., Mousavi, S.M.M., Abraki, S.B., Hadjighassem, M.R., and Gorji, A. (2016). TRPV1 receptors augment basal synaptic transmission in CA1 and CA3 pyramidal neurons in epilepsy. Neuroscience 314, 170-178.

Saiki, R.K., Gelfand, D.H., Stoffel, S., Scharf, S.J., Higuchi, R., Horn, G.T., Mullis, K.B., and Erlich, H.A. (1988). Primer-directed enzymatic amplification of DNA with a thermostable DNA polymerase. Science 239, 487-491.

Salazar, H., Jara-Oseguera, A., Hernández-García, E., Llorente, I., Arias-Olguín, I.I., Soriano-García, M., Islas, L.D., and Rosenbaum, T. (2009). Structural determinants of gating in the TRPV1 channel. Nat. Struct. Mol. Biol. 16, 704-710.

Sallert, M., Rantamäki, T., Vesikansa, A., Anthoni, H., Harju, K., Yli-Kauhaluoma, J., Taira, T., Castren, E., and Lauri, S.E. (2009). Brain-Derived Neurotrophic Factor Controls Activity-Dependent Maturation of CA1 Synapses by Downregulating Tonic Activationof Presynaptic Kainate Receptors. J. Neurosci. 29, 11294-11303.

Samways, D.S.K., and Egan, T.M. (2011). Calcium-dependent decrease in the single channel conductance of TRPV1. Pflugers Arch. 462, 681-691.

Sanabria, E.R.G., Su, H., and Yaari, Y. (2001). Initiation of network bursts by $\mathrm{Ca} 2+-$ dependent intrinsic bursting in the rat pilocarpine model of temporal lobe epilepsy. J. Physiol. 532, 205-216.

Sanchez, J.F., Krause, J.E., and Cortright, D.N. (2001). The distribution and regulation of vanilloid receptor VR1 and VR1 5' splice variant RNA expression in rat. Neuroscience 107, 373-381. 
Sántha, P., Oszlács, O., Dux, M., Dobos, I., and Jancsó, G. (2010). Inhibition of glucosylceramide synthase reversibly decreases the capsaicin-induced activation and TRPV1 expression of cultured dorsal root ganglion neurons. Pain 150, 103112.

Santos, C.J.P.A., Stern, C.A.J., and Bertoglio, L.J. (2008). Attenuation of anxietyrelated behaviour after the antagonism of transient receptor potential vanilloid type 1 channels in the rat ventral hippocampus. Behav. Pharmacol. 19, 357-360.

Sasamura, T., Sasaki, M., Tohda, C., and Kuraishi, Y. (1998). Existence of capsaicin-sensitive glutamatergic terminals in rat hypothalamus. Neuroreport 9, 2045-2048.

Sauer, B. (1987). Functional expression of the cre-lox site-specific recombination system in the yeast Saccharomyces cerevisiae. Mol. Cell. Biol. 7, 2087-2096.

Sauer, B., and Henderson, N. (1988). Site-specific DNA recombination in mammalian cells by the Cre recombinase of bacteriophage P1. Proc. Natl. Acad. Sci. U. S. A. 85, 5166-5170.

Schägger, H., and von Jagow, G. (1987). Tricine-sodium dodecyl sulfatepolyacrylamide gel electrophoresis for the separation of proteins in the range from 1 to $100 \mathrm{kDa}$. Anal. Biochem. 166, 368-379.

Scheibel, M.E., Crandall, P.H., and Scheibel, A.B. (1974). The HippocampalDentate Complex in Temporal Lobe Epilepsy. Epilepsia 15, 55-80.

Schicho, R., Florian, W., Liebmann, I., Holzer, P., and Lippe, I.T. (2004). Increased expression of TRPV1 receptor in dorsal root ganglia by acid insult of the rat gastric mucosa. Eur. J. Neurosci. 19, 1811-1818.

Schmidt-Supprian, M., and Rajewsky, K. (2007). Vagaries of conditional gene targeting. Nat. Immunol. 8, 665-668.

Schnizler, K., Shutov, L.P., Van Kanegan, M.J., Merrill, M.A., Nichols, B., McKnight, G.S., Strack, S., Hell, J.W., and Usachev, Y.M. (2008). Protein kinase A anchoring via AKAP150 is essential for TRPV1 modulation by forskolin and prostaglandin E2 in mouse sensory neurons. J. Neurosci. Off. J. Soc. Neurosci. 28, 4904-4917.

Schultz, W. (1986). Responses of midbrain dopamine neurons to behavioral trigger stimuli in the monkey. J. Neurophysiol. 56, 1439-1461.

Schumacher, M.A., and Eilers, H. (2010). TRPV1 splice variants: structure and function. Front. Biosci. Landmark Ed. 15, 872-882.

Schumacher, M.A., Moff, I., Sudanagunta, S.P., and Levine, J.D. (2000). Molecular Cloning of an N-terminal Splice Variant of the Capsaicin Receptor: LOSS OF N- 
TERMINAL DOMAIN SUGGESTS FUNCTIONAL DIVERGENCE AMONG CAPSAICIN RECEPTOR SUBTYPES. J. Biol. Chem. 275, 2756-2762.

Schwanhäusser, B., Busse, D., Li, N., Dittmar, G., Schuchhardt, J., Wolf, J., Chen, W., and Selbach, M. (2011). Global quantification of mammalian gene expression control. Nature 473, 337-342.

Schwartz, E.S., La, J.-H., Scheff, N.N., Davis, B.M., Albers, K.M., and Gebhart, G.F. (2013). TRPV1 and TRPA1 antagonists prevent the transition of acute to chronic inflammation and pain in chronic pancreatitis. J. Neurosci. Off. J. Soc. Neurosci. 33, 5603-5611.

Sharif-Naeini, R., Ciura, S., and Bourque, C.W. (2008). TRPV1 Gene Required for Thermosensory Transduction and Anticipatory Secretion from Vasopressin Neurons during Hyperthermia. Neuron 58, 179-185.

Shelly, M., Cancedda, L., Heilshorn, S., Sumbre, G., and Poo, M. (2007). LKB1/STRAD Promotes Axon Initiation During Neuronal Polarization. Cell 129, 565-577.

Shibasaki, K., Suzuki, M., Mizuno, A., and Tominaga, M. (2007). Effects of Body Temperature on Neural Activity in the Hippocampus: Regulation of Resting Membrane Potentials by Transient Receptor Potential Vanilloid 4. J. Neurosci. 27, $1566-1575$.

Shigemoto, R., Kulik, A., Roberts, J.D.B., Ohishi, H., Nusser, Z., Kaneko, T., and Somogyi, P. (1996). Target-cell-specific concentration of a metabotropic glutamate receptor in the presynaptic active zone. Nature 381, 523-525.

Shigemoto, R., Kinoshita, A., Wada, E., Nomura, S., Ohishi, H., Takada, M., Flor, P.J., Neki, A., Abe, T., Nakanishi, S., et al. (1997). Differential Presynaptic Localization of Metabotropic Glutamate Receptor Subtypes in the Rat Hippocampus. J. Neurosci. 17, 7503-7522.

Shoback, D.M., and Brown, E.M. (1984). Forskolin increases cellular cyclic adenosine monophosphate content and parathyroid hormone release in dispersed bovine parathyroid cells. Metabolism. 33, 509-514.

Shu, X., and Mendell, L.M. (1999). Nerve growth factor acutely sensitizes the response of adult rat sensory neurons to capsaicin. Neurosci. Lett. 274, 159-162.

Sik, A., Penttonen, M., Ylinen, A., and Buzsaki, G. (1995). Hippocampal CA1 interneurons: an in vivo intracellular labeling study. J. Neurosci. 15, 6651-6665.

Sikand, P., and Premkumar, L.S. (2007). Potentiation of glutamatergic synaptic transmission by protein kinase C-mediated sensitization of TRPV1 at the first sensory synapse. J. Physiol. 581, 631-647. 
Sillito, A.M. (1975). The effectiveness of bicuculline as an antagonist of GABA and visually evoked inhibition in the cat's striate cortex. J. Physiol. 250, 287-304.

Smith, G.D., Gunthorpe, M.J., Kelsell, R.E., Hayes, P.D., Reilly, P., Facer, P., Wright, J.E., Jerman, J.C., Walhin, J.-P., Ooi, L., et al. (2002). TRPV3 is a temperature-sensitive vanilloid receptor-like protein. Nature 418, 186-190.

Smith, P.K., Krohn, R.I., Hermanson, G.T., Mallia, A.K., Gartner, F.H., Provenzano, M.D., Fujimoto, E.K., Goeke, N.M., Olson, B.J., and Klenk, D.C. (1985). Measurement of protein using bicinchoninic acid. Anal. Biochem. 150, 76-85.

Somogyi, P., and Klausberger, T. (2005). Defined types of cortical interneurone structure space and spike timing in the hippocampus. J. Physiol. 562, 9-26.

Spencer, J.L., Waters, E.M., Milner, T.A., and McEwen, B.S. (2008). Estrous cycle regulates activation of hippocampal Akt, LIMK, and neurotrophin receptors in C57BL6 mice. Neuroscience 155, 1106-1119.

Spencer-Segal, J.L., Waters, E.M., Bath, K.G., Chao, M.V., McEwen, B.S., and Milner, T.A. (2011). Distribution of TrkB receptor in the mouse hippocampal formation depends on sex and estrous cycle stage. J. Neurosci. Off. J. Soc. Neurosci. 31, 6780-6790.

Starowicz, K., Maione, S., Cristino, L., Palazzo, E., Marabese, I., Rossi, F., Novellis, V. de, and Marzo, V.D. (2007). Tonic Endovanilloid Facilitation of Glutamate Release in Brainstem Descending Antinociceptive Pathways. J. Neurosci. 27, 13739-13749.

Staruschenko, A., Jeske, N.A., and Akopian, A.N. (2010). Contribution of TRPV1TRPA1 Interaction to the Single Channel Properties of the TRPA1 Channel. J. Biol. Chem. 285, 15167-15177.

Stein, A., Radhakrishnan, A., Riedel, D., Fasshauer, D., and Jahn, R. (2007). Synaptotagmin activates membrane fusion through a $\mathrm{Ca} 2+$-dependent trans interaction with phospholipids. Nat. Struct. Mol. Biol. 14, 904-911.

van der Stelt, M., and Di Marzo, V. (2004). Endovanilloids. Eur. J. Biochem. 271, 1827-1834.

Sternberg, N., and Hamilton, D. (1981). Bacteriophage P1 site-specific recombination. J. Mol. Biol. 150, 467-486.

Stranahan, A.M., Erion, J.R., and Wosiski-Kuhn, M. (2013). Reelin signaling in development, maintenance, and plasticity of neural networks. Ageing Res. Rev. 12, 815-822. 
Sudbury, J.R., Ciura, S., Sharif-Naeini, R., and Bourque, C.W. (2010). Osmotic and thermal control of magnocellular neurosecretory neurons - role of an $\mathrm{N}$-terminal variant of trpv1. Eur. J. Neurosci. 32, 2022-2030.

Sugiura, T., Tominaga, M., Katsuya, H., and Mizumura, K. (2002). Bradykinin Lowers the Threshold Temperature for Heat Activation of Vanilloid Receptor 1. J. Neurophysiol. 88, 544-548.

Sun, F.-J., Guo, W., Zheng, D.-H., Zhang, C.-Q., Li, S., Liu, S.-Y., Yin, Q., Yang, H., and Shu, H.-F. (2012). Increased Expression of TRPV1 in the Cortex and Hippocampus from Patients with Mesial Temporal Lobe Epilepsy. J. Mol. Neurosci. 49, 182-193.

Sun, Y., Nguyen, A.Q., Nguyen, J.P., Le, L., Saur, D., Choi, J., Callaway, E.M., and $\mathrm{Xu}, \mathrm{X}$. (2014). Cell-Type-Specific Circuit Connectivity of Hippocampal CA1 Revealed through Cre-Dependent Rabies Tracing. Cell Rep. 7, 269-280.

Sutton, R.B., Davletov, B.A., Berghuis, A.M., Sudhof, T.C., and Sprang, S.R. (1995). Structure of the first C2 domain of synaptotagmin I: A novel $\mathrm{Ca} 2+$ /phospholipid-binding fold. Cell 80, 929-938.

Sylwestrak, E.L., and Ghosh, A. (2012). Elfn1 Regulates Target-Specific Release Probability at CA1-Interneuron Synapses. Science 338, 536-540.

Szallasi, A., Nilsson, S., Farkas-Szallasi, T., Blumberg, P.M., Hökfelt, T., and Lundberg, J.M. (1995). Vanilloid (capsaicin) receptors in the rat: distribution in the brain, regional differences in the spinal cord, axonal transport to the periphery, and depletion by systemic vanilloid treatment. Brain Res. 703, 175-183.

Szallasi, A., Cortright, D.N., Blum, C.A., and Eid, S.R. (2007). The vanilloid receptor TRPV1: 10 years from channel cloning to antagonist proof-of-concept. Nat. Rev. Drug Discov. 6, 357-372.

Szelényi, Z., Hummel, Z., Szolcsányi, J., and Davis, J.B. (2004). Daily body temperature rhythm and heat tolerance in TRPV1 knockout and capsaicin pretreated mice. Eur. J. Neurosci. 19, 1421-1424.

Szolcsányi, J. (1977). A pharmacological approach to elucidation of the role of different nerve fibres and receptor endings in mediation of pain. J. Physiol. (Paris) 73, 251-259.

Takamori, S., Rhee, J.S., Rosenmund, C., and Jahn, R. (2000). Identification of a vesicular glutamate transporter that defines a glutamatergic phenotype in neurons. Nature 407, 189-194.

Tallent, M.K., and Siggins, G.R. (1997). Somatostatin Depresses Excitatory but not Inhibitory Neurotransmission in Rat CA1 Hippocampus. J. Neurophysiol. 78, 30083018. 
Tanaka, J., Horiike, Y., Matsuzaki, M., Miyazaki, T., Ellis-Davies, G.C.R., and Kasai, H. (2008). Protein Synthesis and Neurotrophin-Dependent Structural Plasticity of Single Dendritic Spines. Science 319, 1683-1687.

Tao, X., Finkbeiner, S., Arnold, D.B., Shaywitz, A.J., and Greenberg, M.E. (1998). $\mathrm{Ca} 2+$ Influx Regulates BDNF Transcription by a CREB Family Transcription Factor-Dependent Mechanism. Neuron 20, 709-726.

Tao, X., West, A.E., Chen, W.G., Corfas, G., and Greenberg, M.E. (2002). A Calcium-Responsive Transcription Factor, CaRF, that Regulates Neuronal ActivityDependent Expression of BDNF. Neuron 33, 383-395.

Teng, K.K., Felice, S., Kim, T., and Hempstead, B.L. (2010). Understanding Proneurotrophin Actions: Recent Advances and Challenges. Dev. Neurobiol. 70, 350-359.

Thoenen, H. (1995). Neurotrophins and Neuronal Plasticity. Science 270, 593-598.

Thresh, C.F. (1876). Capsaicin, the active principle of capsicum fruits. Analyst 1, $148 b-149$.

Tian, W., Fu, Y., Wang, D.H., and Cohen, D.M. (2006). Regulation of TRPV1 by a novel renally expressed rat TRPV1 splice variant. Am. J. Physiol. - Ren. Physiol. 290, F117-F126.

Tian, Y.-H., Lee, S.-Y., Kim, H.-C., and Jang, C.-G. (2010). Repeated methamphetamine treatment increases expression of TRPV1 mRNA in the frontal cortex but not in the striatum or hippocampus of mice. Neurosci. Lett. 472, 61-64.

Tominaga, M., and Caterina, M.J. (2004). Thermosensation and pain. J. Neurobiol. $61,3-12$.

Tominaga, M., Caterina, M.J., Malmberg, A.B., Rosen, T.A., Gilbert, H., Skinner, K., Raumann, B.E., Basbaum, A.I., and Julius, D. (1998). The Cloned Capsaicin Receptor Integrates Multiple Pain-Producing Stimuli. Neuron 21, 531-543.

Tomioka, N.H., Yasuda, H., Miyamoto, H., Hatayama, M., Morimura, N., Matsumoto, Y., Suzuki, T., Odagawa, M., Odaka, Y.S., Iwayama, Y., et al. (2014). Elfn1 recruits presynaptic mGluR7 in trans and its loss results in seizures. Nat. Commun. 5, 4501.

Tonra, J.R., Curtis, R., Wong, V., Cliffer, K.D., Park, J.S., Timmes, A., Nguyen, T., Lindsay, R.M., Acheson, A., and DiStefano, P.S. (1998). Axotomy Upregulates the Anterograde Transport and Expression of Brain-Derived Neurotrophic Factor by Sensory Neurons. J. Neurosci. 18, 4374-4383. 
Tort, A.B.L., Rotstein, H.G., Dugladze, T., Gloveli, T., and Kopell, N.J. (2007). On the formation of gamma-coherent cell assemblies by oriens lacunosum-moleculare interneurons in the hippocampus. Proc. Natl. Acad. Sci. 104, 13490-13495.

Tóth, A., Boczán, J., Kedei, N., Lizanecz, E., Bagi, Z., Papp, Z., Édes, I., Csiba, L., and Blumberg, P.M. (2005). Expression and distribution of vanilloid receptor 1 (TRPV1) in the adult rat brain. Mol. Brain Res. 135, 162-168.

Tóth, A., Blumberg, P.M., and Boczán, J. (2009). Chapter 15 Anandamide and the Vanilloid Receptor (TRPV1). B.-V.\& Hormones, ed. (Academic Press), pp. 389419.

Tóth, D.M., Szőke, É., Bölcskei, K., Kvell, K., Bender, B., Bősze, Z., Szolcsányi, J., and Sándor, Z. (2010). Nociception, neurogenic inflammation and thermoregulation in TRPV1 knockdown transgenic mice. Cell. Mol. Life Sci. 68, 2589-2601.

Towbin, H., Staehelin, T., and Gordon, J. (1979). Electrophoretic transfer of proteins from polyacrylamide gels to nitrocellulose sheets: procedure and some applications. Proc. Natl. Acad. Sci. U. S. A. 76, 4350-4354.

Ugarte, G.D., Diaz, E., Biscaia, M., Stehberg, J., Montecino, M., and van Zundert, B. (2013). Transcription of the pain-related TRPV1 gene requires Runx1 and C/EBP $\beta$ factors. J. Cell. Physiol. 228, 860-870.

Valente, P., García-Sanz, N., Gomis, A., Fernández-Carvajal, A., FernándezBallester, G., Viana, F., Belmonte, C., and Ferrer-Montiel, A. (2008). Identification of molecular determinants of channel gating in the transient receptor potential box of vanilloid receptor I. FASEB J. Off. Publ. Fed. Am. Soc. Exp. Biol. 22, 32983309 .

Vanecko, S., and Laskowski, M. (1961). Studies of the specificity of deoxyribonuclease I. III. Hydrolysis of chains carrying a monoesterified phosphate on carbon 5'. J. Biol. Chem. 236, 3312-3316.

Varga, A., Németh, J., Szabó, Á., McDougall, J.J., Zhang, C., Elekes, K., Pintér, E., Szolcsányi, J., and Helyes, Z. (2005). Effects of the novel TRPV1 receptor antagonist SB366791 in vitro and in vivo in the rat. Neurosci. Lett. 385, 137-142.

Vicario-Abejón, C., Collin, C., McKay, R.D.G., and Segal, M. (1998). Neurotrophins Induce Formation of Functional Excitatory and Inhibitory Synapses between Cultured Hippocampal Neurons. J. Neurosci. 18, 7256-7271.

Vincent, F., and A.J. Duncton, M. (2011). TRPV4 Agonists and Antagonists. Curr. Top. Med. Chem. 11, 2216-2226.

Vlachová, V., Teisinger, J., Sušánková, K., Lyfenko, A., Ettrich, R., and Vyklický, L. (2003). Functional Role of C-Terminal Cytoplasmic Tail of Rat Vanilloid Receptor 1. J. Neurosci. 23, 1340-1350. 
Vogel, C., and Marcotte, E.M. (2012). Insights into the regulation of protein abundance from proteomic and transcriptomic analyses. Nat. Rev. Genet. 13, 227232.

Vriens, J., Nilius, B., and Vennekens, R. (2008). Herbal Compounds and Toxins Modulating TRP Channels. Curr. Neuropharmacol. 6, 79-96.

Vriens, J., Appendino, G., and Nilius, B. (2009). Pharmacology of Vanilloid Transient Receptor Potential Cation Channels. Mol. Pharmacol. 75, 1262-1279.

Walker, K.M., Urban, L., Medhurst, S.J., Patel, S., Panesar, M., Fox, A.J., and Mclntyre, P. (2003). The VR1 Antagonist Capsazepine Reverses Mechanical Hyperalgesia in Models of Inflammatory and Neuropathic Pain. J. Pharmacol. Exp. Ther. 304, 56-62.

Walpole, C.S.J., Bevan, S., Bovermann, G., Boelsterli, J.J., Breckenridge, R., Davies, J.W., Hughes, G.A., James, I., and Oberer, L. (1994). The Discovery of Capsazepine, the First Competitive Antagonist of the Sensory Neuron Excitants Capsaicin and Resiniferatoxin. J. Med. Chem. 37, 1942-1954.

Wang, C., Hu, H.-Z., Colton, C.K., Wood, J.D., and Zhu, M.X. (2004). An Alternative Splicing Product of the Murine trpv1 Gene Dominant Negatively Modulates the Activity of TRPV1 Channels. J. Biol. Chem. 279, 37423-37430.

Wang, S., Davis, B.M., Zwick, M., Waxman, S.G., and Albers, K.M. (2006). Reduced thermal sensitivity and Nav1.8 and TRPV1 channel expression in sensory neurons of aged mice. Neurobiol. Aging 27, 895-903.

Ward, J.M., and Rehg, J.E. (2014). Rodent Immunohistochemistry Pitfalls and Troubleshooting. Vet. Pathol. Online 51, 88-101.

Weng, D.Y., Zhang, Y., Hayashi, Y., Kuan, C.-Y., Liu, C.-Y., Babcock, G., Weng, W.-L., Schwemberger, S., and Kao, W.W.-Y. (2008). Promiscuous recombination of LoxP alleles during gametogenesis in cornea Cre driver mice. Mol. Vis. 14, 562 571.

Wetmore, C., and Olson, L. (1995). Neuronal and nonneuronal expression of neurotrophins and their receptors in sensory and sympathetic ganglia suggest new intercellular trophic interactions. J. Comp. Neurol. 353, 143-159.

White, J.A., Banks, M.I., Pearce, R.A., and Kopell, N.J. (2000). Networks of interneurons with fast and slow $y$-aminobutyric acid type A (GABAA) kinetics provide substrate for mixed gamma-theta rhythm. Proc. Natl. Acad. Sci. 97, 81288133.

Widmer, H., Ferrigan, L., Davies, C. h., and Cobb, S. r. (2006). Evoked slow muscarinic acetylcholinergic synaptic potentials in rat hippocampal interneurons. Hippocampus 16, 617-628. 
Wiechelman, K.J., Braun, R.D., and Fitzpatrick, J.D. (1988). Investigation of the bicinchoninic acid protein assay: Identification of the groups responsible for color formation. Anal. Biochem. 175, 231-237.

Will, T.J., Tushev, G., Kochen, L., Nassim-Assir, B., Cajigas, I.J., Dieck, S. tom, and Schuman, E.M. (2013). Deep Sequencing and High-Resolution Imaging Reveal Compartment-Specific Localization of Bdnf mRNA in Hippocampal Neurons. Sci. Signal. 6, rs16-rs16.

Winston, J., Toma, H., Shenoy, M., and Pasricha, P.J. (2001). Nerve growth factor regulates VR-1 mRNA levels in cultures of adult dorsal root ganglion neurons: Pain 89, 181-186.

Winter, J., Dray, A., Wood, J.N., Yeats, J.C., and Bevan, S. (1990). Cellular mechanism of action of resiniferatoxin: a potent sensory neuron excitotoxin. Brain Res. 520, 131-140.

Woo, N.H., Teng, H.K., Siao, C.-J., Chiaruttini, C., Pang, P.T., Milner, T.A., Hempstead, B.L., and Lu, B. (2005). Activation of p75NTR by proBDNF facilitates hippocampal long-term depression. Nat. Neurosci. 8, 1069-1077.

Woolf, C.J., and Costigan, M. (1999). Transcriptional and posttranslational plasticity and the generation of inflammatory pain. Proc. Natl. Acad. Sci. U. S. A. 96, 7723-7730.

Wu, L.-J., Sweet, T.-B., and Clapham, D.E. (2010). International Union of Basic and Clinical Pharmacology. LXXVI. Current Progress in the Mammalian TRP Ion Channel Family. Pharmacol. Rev. 62, 381-404.

Wulff, P., Ponomarenko, A.A., Bartos, M., Korotkova, T.M., Fuchs, E.C., Bähner, F., Both, M., Tort, A.B.L., Kopell, N.J., Wisden, W., et al. (2009). Hippocampal theta rhythm and its coupling with gamma oscillations require fast inhibition onto parvalbumin-positive interneurons. Proc. Natl. Acad. Sci. 106, 3561-3566.

Xia, C.-M., Gulick, M.A., Yu, S.J., Grider, J.R., Murthy, K.S., Kuemmerle, J.F., Akbarali, H.I., and Qiao, L.-Y. (2012). Up-regulation of brain-derived neurotrophic factor in primary afferent pathway regulates colon-to-bladder cross-sensitization in rat. J. Neuroinflammation 9, 30.

Xing, J., and Li, J. (2007). TRPV1 Receptor Mediates Glutamatergic Synaptic Input to Dorsolateral Periaqueductal Gray (dl-PAG) Neurons. J. Neurophysiol. 97, 503511.

Xue, Q., Yu, Y., Trilk, S.L., Jong, B.E., and Schumacher, M.A. (2001). The Genomic Organization of the Gene Encoding the Vanilloid Receptor: Evidence for Multiple Splice Variants. Genomics 76, 14-20. 
Xue, Q., Jong, B., Chen, T., and Schumacher, M.A. (2007). Transcription of rat TRPV1 utilizes a dual promoter system that is positively regulated by nerve growth factor. J. Neurochem. 101, 212-222.

Yajima, Y., Narita, M., Narita, M., Matsumoto, N., and Suzuki, T. (2002). Involvement of a spinal brain-derived neurotrophic factor/full-length TrkB pathway in the development of nerve injury-induced thermal hyperalgesia in mice. Brain Res. 958, 338-346.

Yang, F., Cui, Y., Wang, K., and Zheng, J. (2010). Thermosensitive TRP channel pore turret is part of the temperature activation pathway. Proc. Natl. Acad. Sci. U. S. A. $107,7083-7088$.

Yang, J., Siao, C.-J., Nagappan, G., Marinic, T., Jing, D., McGrath, K., Chen, Z.-Y., Mark, W., Tessarollo, L., Lee, F.S., et al. (2009). Neuronal release of proBDNF. Nat. Neurosci. 12, 113-115.

Yang, K., Kumamoto, E., Furue, H., and Yoshimura, M. (1998). Capsaicin facilitates excitatory but not inhibitory synaptic transmission in substantia gelatinosa of the rat spinal cord. Neurosci. Lett. 255, 135-138.

Yaziji, H., and Barry, T. (2006). Diagnostic Immunohistochemistry: What can Go Wrong?: Adv. Anat. Pathol. 13, 238-246.

Yoshida, T., Inoue, R., Morii, T., Takahashi, N., Yamamoto, S., Hara, Y., Tominaga, M., Shimizu, S., Sato, Y., and Mori, Y. (2006). Nitric oxide activates TRP channels by cysteine S-nitrosylation. Nat. Chem. Biol. 2, 596-607.

You, I.-J., Jung, Y.-H., Kim, M.-J., Kwon, S.-H., Hong, S.-I., Lee, S.-Y., and Jang, C.-G. (2012). Alterations in the emotional and memory behavioral phenotypes of transient receptor potential vanilloid type 1-deficient mice are mediated by changes in expression of 5-HT1A, GABAA, and NMDA receptors. Neuropharmacology 62, 1034-1043.

Zachrisson, O., Falkenberg, T., and Lindefors, N. (1996). Neuronal coexistence of trkB and glutamic acid decarboxylase 67 mRNAs in rat hippocampus. Mol. Brain Res. 36, 169-173.

Zafra, F., Hengerer, B., Leibrock, J., Thoenen, H., and Lindholm, D. (1990). Activity dependent regulation of BDNF and NGF mRNAs in the rat hippocampus is mediated by non-NMDA glutamate receptors. EMBO J. 9, 3545-3550.

Zafra, F., Lindholm, D., Castren, E., Hartikka, J., and Thoenen, H. (1992). Regulation of brain-derived neurotrophic factor and nerve growth factor mRNA in primary cultures of hippocampal neurons and astrocytes. J. Neurosci. 12, 47934799. 


\section{BIBLIOGRAPHY}

Zakharenko, S.S., Patterson, S.L., Dragatsis, I., Zeitlin, S.O., Siegelbaum, S.A., Kandel, E.R., and Morozov, A. (2003). Presynaptic BDNF Required for a Presynaptic but Not Postsynaptic Component of LTP at Hippocampal CA1-CA3 Synapses. Neuron 39, 975-990.

Zhang, X., Huang, J., and McNaughton, P.A. (2005). NGF rapidly increases membrane expression of TRPV1 heat-gated ion channels. EMBO J. 24, 42114223.

Zhao, J., Seereeram, A., Nassar, M.A., Levato, A., Pezet, S., Hathaway, G., Morenilla-Palao, C., Stirling, C., Fitzgerald, M., McMahon, S.B., et al. (2006). Nociceptor-derived brain-derived neurotrophic factor regulates acute and inflammatory but not neuropathic pain. Mol. Cell. Neurosci. 31, 539-548.

Zhou, H.-Y., Zhang, H.-M., Chen, S.-R., and Pan, H.-L. (2007). Increased Nociceptive Input Rapidly Modulates Spinal GABAergic Transmission Through Endogenously Released Glutamate. J. Neurophysiol. 97, 871-882.

Zygmunt, P.M., Petersson, J., Andersson, D.A., Chuang, H., Sørgård, M., Di Marzo, V., Julius, D., and Högestätt, E.D. (1999). Vanilloid receptors on sensory nerves mediate the vasodilator action of anandamide. Nature 400, 452-457.

Zygmunt, P.M., Ermund, A., Movahed, P., Andersson, D.A., Simonsen, C., Jönsson, B.A.G., Blomgren, A., Birnir, B., Bevan, S., Eschalier, A., et al. (2013). Monoacylglycerols Activate TRPV1 - A Link between Phospholipase C and TRPV1. PLoS ONE 8, e81618. 


\section{Curriculum vitae}

\begin{tabular}{|c|c|}
\hline Surname: & Hurtado Zavala \\
\hline First Name: & Joaquín Isaac \\
\hline Date of birth: & 19.11.1986 \\
\hline Place of birth: & Monterrey, Nuevo León, Mexico \\
\hline Nationality: & Mexican \\
\hline \multirow[t]{2}{*}{ Home address: } & Albrecht-von-Haller Str. 2 \\
\hline & 37075 Göttingen, Germany \\
\hline \multirow[t]{4}{*}{ Work address: } & ENI-Göttingen \\
\hline & Trans-synaptic signaling group \\
\hline & Grisebachstr. 5 \\
\hline & 37077, Göttingen, Germany. \\
\hline E-mail: & joacolatoso@hotmail.com \\
\hline EDUCATION & \\
\hline
\end{tabular}

April. 2016 PhD in the Grundprogramm Biologie at Georg-August University School of Science (GAUSS), Germany. "TRPV1 regulates excitatory innervation of oriens lacunosum moleculare (OLM) neurons in the hippocampus to affect synaptic plasticity" (supervisor: Camin Dean, PhD)

May 2011 M.Sc. in Developmental, Neural, and Behavioral Biology at the University of Göttingen, Germany. "Homeostatic plasticity in the hippocampus: from single neurons to neuronal networks" (supervisor: Camin Dean, PhD)

May 2009 B.Sc. in Biology at the Universidad Autónoma de Nuevo León, Mexico. "Identification of a circadian rhythmicity in the agonistic behavior of the marbled crayfish Procambarus sp (Ortmann, 1905)" (supervisors: Prof. Dr. Juan Homero López Soto (UANL) and Prof. Dr. Ralf Heinrich at the University of Göttingen). 
Languages: Spanish: native

English: fluent

German: C1

Italian: B1

SCHOLARSHIPS AWARDED:

2009-2010 CONACYT-DAAD scholarship.

2006-2007 DAAD-Exchange program UANL-Uni Göttingen.

PUBLICATIONS:

Farca Luna, A.J., Hurtado-Zavala, J.I., Reischig, T., and Heinrich, R. (2009). Circadian regulation of agonistic behavior in groups of parthenogenetic marbled crayfish, Procambarus sp. J. Biol. Rhythms 24, 64-72. 